ABSTRACT

Title of Document:

HIGH-PERFORMANCE 3D IMAGE PROCESSING ARCHITECTURES FOR IMAGE-GUIDED INTERVENTIONS

Omkar Dandekar, Ph.D., 2008

Directed By:

Professor Raj Shekhar (Chair/Advisor), Professor Shuvra S. Bhattacharyya (Co-advisor), Department of Electrical and Computer Engineering

Minimally invasive image-guided interventions (IGIs) are time and cost efficient, minimize unintended damage to healthy tissues, and lead to faster patient recovery. Advanced three-dimensional (3D) image processing is a critical need for navigation during IGIs. However, achieving on-demand performance, as required by IGIs, for these image processing operations using software-only implementations is challenging because of the sheer size of the 3D images, and memory and compute intensive nature of the operations. This dissertation, therefore, is geared toward developing high-performance 3D image processing architectures, which will enable improved intraprocedural visualization and navigation capabilities during IGIs.

In this dissertation we present an architecture for real-time implementation of 3D filtering operations that are commonly employed for preprocessing of medical images. This architecture is approximately two orders of magnitude faster than 
corresponding software implementations and is capable of processing 3D medical images at their acquisition speeds.

Combining complementary information through registration between pre- and intraprocedural images is a fundamental need in the IGI workflow. Intensity-based deformable registration, which is completely automatic and locally accurate, is a promising approach to achieve this alignment. These algorithms, however, are extremely compute intensive, which has prevented their clinical use. We present an FPGA-based architecture for accelerated implementation of intensity-based deformable image registration. This high-performance architecture achieves over an order of magnitude speedup when compared with a corresponding software implementation and reduces the execution time of deformable registration from hours to minutes while offering comparable image registration accuracy.

Furthermore, we present a framework for multiobjective optimization of finite-precision implementations of signal processing algorithms that takes into account multiple conflicting objectives such as implementation accuracy and hardware resource consumption. The evaluation that we have performed in the context of FPGA-based image registration demonstrates that such an analysis can be used to enhance automated hardware design processes, and efficiently identify a system configuration that meets given design constraints. In addition, we also outline two novel clinical applications that can directly benefit from these developments and demonstrate the feasibility of our approach in the context of these applications. These advances will ultimately enable integration of 3D image processing into clinical workflow. 


\title{
HIGH-PERFORMANCE 3D IMAGE PROCESSING ARCHITECTURES FOR IMAGE-GUIDED INTERVENTIONS
}

By

\author{
Omkar Dandekar
}
Dissertation submitted to the Faculty of the Graduate School of the University of Maryland, College Park, in partial fulfillment of the requirements for the degree of Doctor of Philosophy 2008

Advisory Committee:

Professor Raj Shekhar, Chair/Advisor

Professor Shuvra S. Bhattacharyya, Co-advisor

Professor Rama Chellappa

Professor Manoj Franklin

Professor Yang Tao 
(C) Copyright by Omkar Dandekar 


\section{Dedication}

To my Mother, Mangal, Himani, and the memories of my late Father, for their love and support 


\section{Publications}

- W. Plishker, O. Dandekar, S. Bhattacharyya, and R. Shekhar, "Towards a heterogeneous medical image registration acceleration platform," IEEE Transactions on Biomedical Circuits and Systems, (in preparation), 2008.

- R. Shekhar, O. Dandekar, V. Bhat, R. Mezrich, and A. Park, "Development of CT-guided minimally invasive surgery," Surgical Innovation, (in preparation), 2008.

- O. Dandekar, W. Plishker, S. S. Bhattacharyya, and R. Shekhar, "Multiobjective optimization for reconfigurable implementation of medical image registration," International Journal of Reconfigurable Computing, (under review), 2008.

- P. Lei, O. Dandekar, D. Widlus, P. Malloy, and R. Shekhar, "Incorporation of PET into CT-guided liver radiofrequency ablation,” Radiology, (under revision), 2008.

- O. Dandekar, W. Plishker, S. S. Bhattacharyya, and R. Shekhar, "Multiobjective optimization of FPGA-based medical image registration," presented at IEEE Symposium on Field-Programmable Custom Computing Machines, 2008.

- O. Dandekar and R. Shekhar, "FPGA-accelerated deformable image registration for improved target-delineation during CT-guided interventions," IEEE Transactions on Biomedical Circuits and Systems, vol. 1 (2), 2007, pp. $116-127$. 
- O. Dandekar, C. Castro-Pareja, and R. Shekhar, "FPGA-based real-time 3D image preprocessing for image-guided medical interventions," Journal of Real-Time Image Processing, vol. 1 (4), pp. 285-301, 2007.

- W. Plishker, O. Dandekar, S. Bhattacharyya, and R. Shekhar, "Towards a heterogeneous medical image registration acceleration platform," presented at IEEE Biomedical Circuits and Systems Conference, 2007, pp. 231-234.

- O. Dandekar, K. Siddiqui, V. Walimbe, and R. Shekhar, "Image registration accuracy with low-dose CT: How low can we go?,” presented at IEEE International Symposium on Biomedical Imaging, 2006, pp. 502-505.

- C. R. Castro-Pareja, O. Dandekar, and R. Shekhar, "FPGA-based real-time anisotropic diffusion filtering of 3D ultrasound images,” in SPIE Real-Time Imaging, 2005, pp. 123-131.

- S. Venugopal, C. R. Castro-Pareja, and O. Dandekar, "An FPGA-based 3D image processor with median and convolution filters for real-time applications,” in SPIE Real-Time Imaging, 2005, pp. 174-182. 


\section{Acknowledgements}

I would like to express my sincere gratitude to Dr. Raj Shekhar for his guidance and financial support throughout my graduate education at The Ohio State University, the Cleveland Clinic, and University of Maryland. He has been the perfect mentor for my doctoral research, and a person from whom I have learnt a lot during the past five years. He ensured that I master not only the intricacies of medical image processing, but also put a strong emphasis on developing the qualities necessary for

effective dissemination of scientific research and results. Beyond doubt, he has played the most important role in shaping my technical writing and presentation skills. All throughout my graduate career he has made himself available at any moment when I needed his inputs and feedback for my work or anything else. The time spent working with Dr. Shekhar has truly been the most rewarding career experience of my life.

I would also like to thank my dissertation committee members, Prof. Shuvra Bhattacharyya, Prof. Rama Chellappa, Prof. Manoj Franklin, and Prof Yang Tao for their cooperation and support. I would especially like to thank Prof. Bhattacharyya for his help and collaboration in my work that resulted in an important chapter of this dissertation. The research work reported in this dissertation was partly supported by U.S. Department of Defense (TATRC) under grant DAMD17-03-2-0001.

My research at the University of Maryland would not have been possible without the support and encouragement of Drs. Rueben Mezrich, Adrian Park, Eliot Siegel, Khan Siddiqui, Nancy Knight, Faaiza Mahmoud, Steve Kavic, and all clinical staff at the University of Maryland and Baltimore VA. Whenever I 
requested, they have spared valuable time from their busy schedules for discussions with me, and have been immensely helpful especially during the clinical validation studies. Dr. Siddiqui, in particular, was instrumental in providing clinical perspective on some of the research problems I have explored.

I would like to thank Prof. Jogikal Jagadeesh for allowing me to work in his lab during my first two years at the Ohio State University, and for his crucial guidance during early stages of my graduate education. I am also thankful to Dr. Carlos R. Castro-Pareja, Dr Vivek Walimbe, Dr William Plishker, Dr. Jianzhou Wu, Peng Lei, and Venkatesh Bhat from Dr. Shekhar's research group for providing valuable help and inputs at various times during my research.

My parents have always been the biggest source of inspiration in my life. They have always stressed the importance of education and instilled in me the virtues of honest and dedicated effort, for which I will forever be indebted to them. Their love and constant encouragement has been an important driving force throughout my life. I would like to thank my sister, Mangal, for her kind words of encouragement from time to time during the last few years. I would like to especially mention my long time friends Mukta, Prashant, Sandip, Siddharth, Rahul, Rakhi, and Vinayak, for always being there with me.

Last, and most importantly, I would like to thank Himani - my wife and my best friend. She has shown incredible patience and understanding throughout the course of my graduate studies. I would not have been able to successfully complete my doctoral program without the constant encouragement and motivation she provided. My achievements are my tribute to her unconditional love and support. 


\section{Table of Contents}

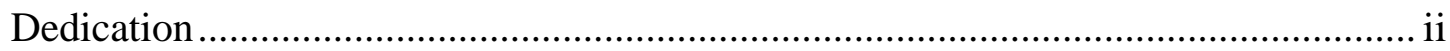

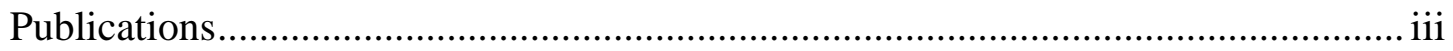

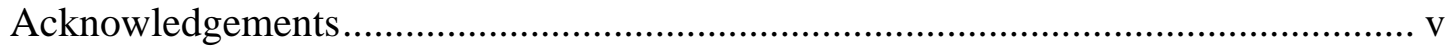

Table of Contents ................................................................................................ vii

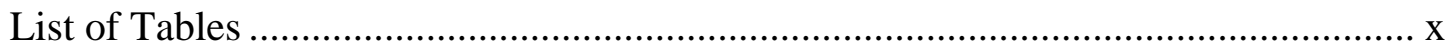

List of Figures ................................................................................................. xii

Chapter 1: Introduction ...................................................................................... 1

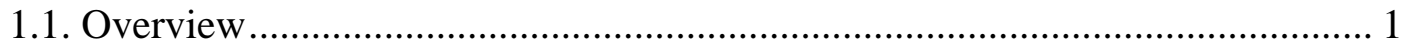

1.2. Contributions of this Dissertation ................................................................. 3

1.2.1. Real-time 3D Image Preprocessing ............................................................. 4

1.2.2. Hardware-Accelerated Deformable Image Registration................................ 5

1.2.3. Framework for Optimization of Finite Precision Implementations .............. 6

1.3. Outline of this Dissertation ................................................................................ 7

Chapter 2: Background and Related Work ............................................................. 9

2.1. Image-Guided Interventions ........................................................................ 9

2.1.1. Role of Preprocedural Imaging ............................................................. 10

2.1.2. Need for Image Registration.................................................................... 12

2.2. Classification of Image Registration.............................................................. 13

2.2.1. Image Registration using Extrinsic Information........................................ 14

2.2.2. Image Registration using Intrinsic Information......................................... 15

2.3. Intensity-Based Image Registration ............................................................... 17

2.3.1. Transformation Models......................................................................... 18

2.3.2. Image Similarity Measures ………………………………………….... 22

2.3.3. Optimization Algorithms .................................................................... 26

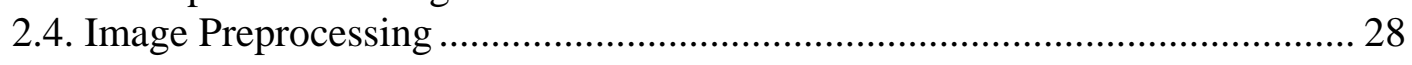

2.4.1. Anisotropic Diffusion Filtering................................................................. 30

2.4.2. Median Filtering....................................................................................... 31

2.5. Optimization of Finite Precision Implementations ............................................ 32

2.5.1. Optimal Wordlength Formulation............................................................... 33

2.5.2. Simulation-Based Optimal Wordlength Search........................................... 34

2.5.3. Multiobjective Optimization...................................................................... 35

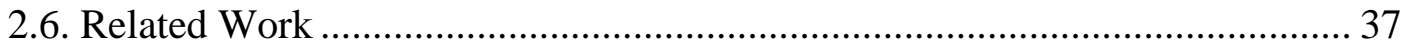

2.6.1. Real-Time Image Preprocessing ............................................................. 37

2.6.2. Acceleration of Image Registration ............................................................ 40

2.6.3. Optimization of Finite Precision Implementations ..................................... 43

Chapter 3: Real-time 3D Image Processing.............................................................. 47

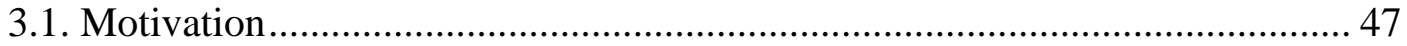

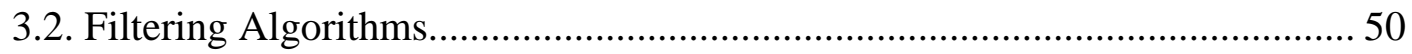

3.2.1. Anisotropic Diffusion Filtering............................................................... 50

3.2.2. Median Filtering....................................................................................... 51

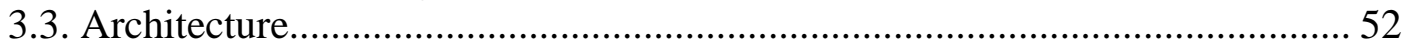


3.3.1. Memory Controller and Brick-caching Scheme ......................................... 54

3.3.2. 3D Anisotropic Diffusion Filtering........................................................... 58

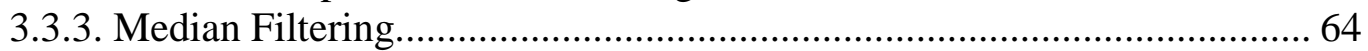

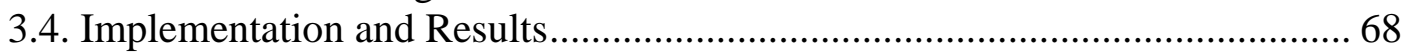

3.4.1. Effects of Finite Precision Representation................................................... 69

3.4.2. Hardware Requirements.......................................................................... 73

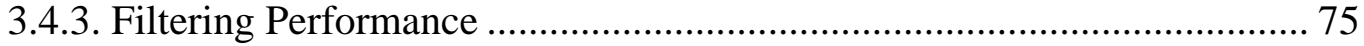

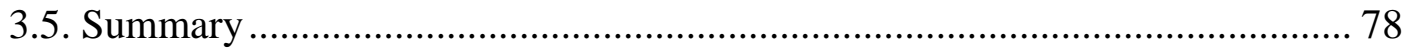

Chapter 4: Hardware-Accelerated Deformable Image Registration............................ 80

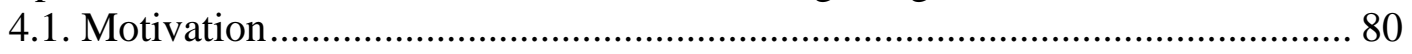

4.2. Algorithm for Deformable Image Registration................................................. 83

4.2.1. Calculating MI for a Subvolume............................................................. 85

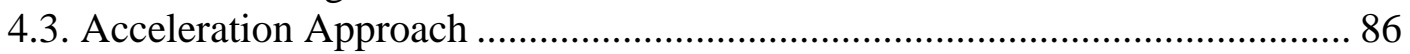

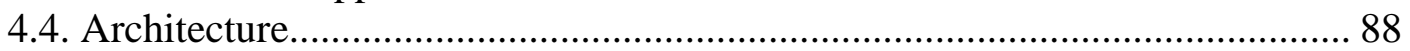

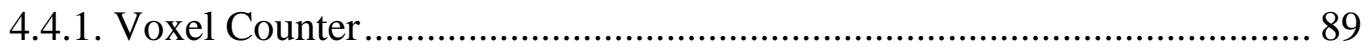

4.4.2. Coordinate Transformation..................................................................... 90

4.4.3. Partial Volume Interpolation................................................................... 92

4.4.4. Image Memory Access ………………………………………………...... 94

4.4.5. Updating Mutual Histogram .................................................................... 99

4.4.6. Entropy Calculation ............................................................................. 105

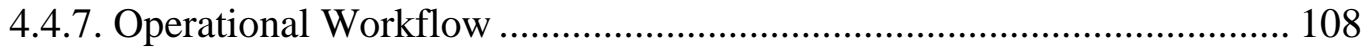

4.5. Implementation and Results....................................................................... 111

4.5.1. Execution Speed................................................................................... 114

4.5.2. Performance Comparison........................................................................ 117

4.5.3. Qualitative Evaluation of Deformable Image Registration ..................... 122

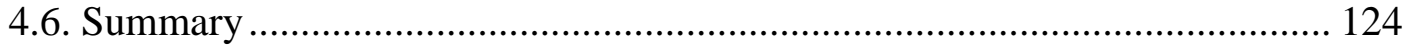

Chapter 5: Framework for Optimization of Finite Precision Implementations ......... 126

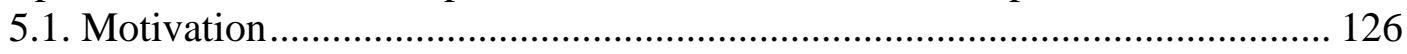

5.2. Multiobjective Optimization ......................................................................... 129

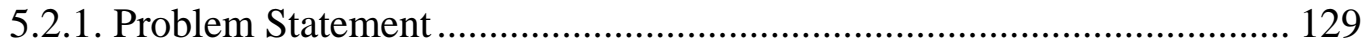

5.2.2. Parameterized Architectural Design ....................................................... 131

5.2.3. Multiobjective Optimization Framework ................................................ 134

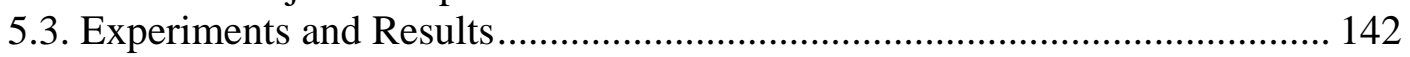

5.3.1. Metrics for Comparison of Pareto-optimized Solution Sets .................... 146

5.3.2. Accuracy of Image Registration ............................................................ 148

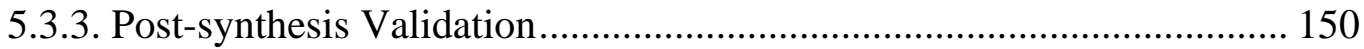

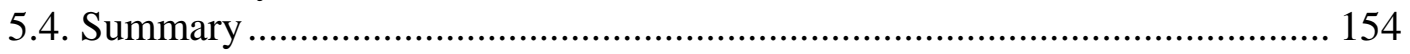

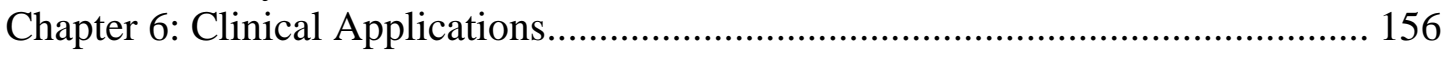

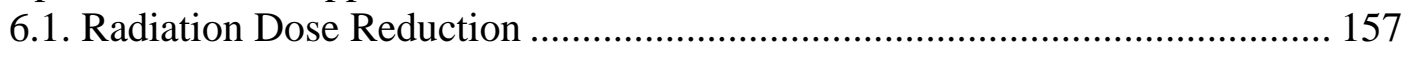

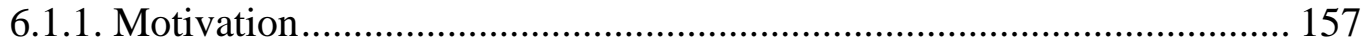

6.1.2. Dose Reduction Strategy....................................................................... 158

6.1.3. Evaluation of Registration Accuracy with Low-Dose CT ....................... 159

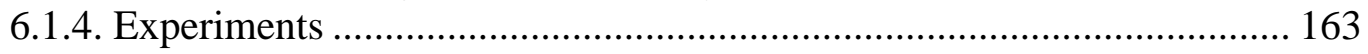

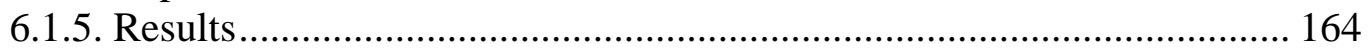

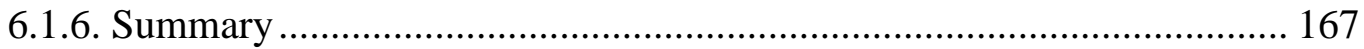

6.2. Incorporation of PET into CT-Guided Liver Radio-Frequency Ablation ..... 168 


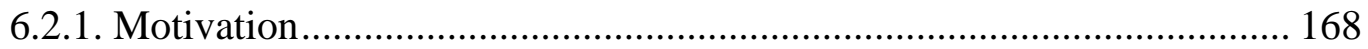

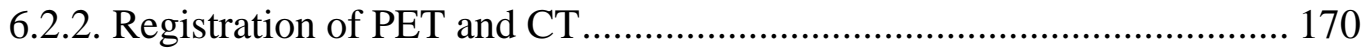

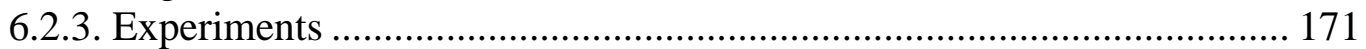

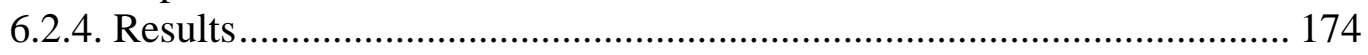

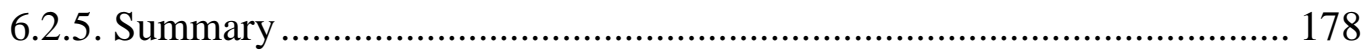

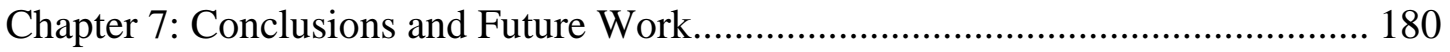

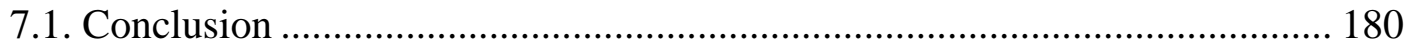

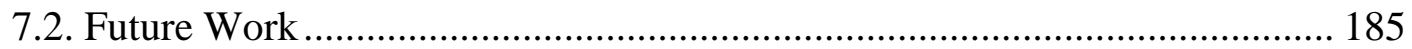

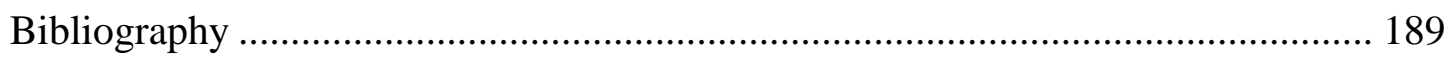




\section{List of Tables}

Table 2.1: $\quad$ Broad classification of image registration in the context of IGI......... 13

Table 3.1: $\quad$ Software execution time of 3D anisotropic diffusion filtering and 3D median filtering of 8-bit images for common kernel sizes $(N)$. 48

Table 3.2: $\quad$ Average error in intensity per voxel for a Gaussian filtered image resulting from fixed-point representation of Gaussian coefficients.... 69

Table 3.3: $\quad$ Average error per sample of diffusion function resulting from fixedpoint representation of diffusion coefficients employed in the presented architecture. 70

Table 3.4: Average error in intensity per voxel for anisotropic diffusion filtered resulting from fixed-point representation of Gaussian coefficients and the diffusion function.

Table 3.5: Hardware requirements of the architecture for real-time 3D image preprocessing.

Table 3.6: Hardware requirements for the components of the linear systolic implementation of the 3D median filtering. 74

Table 3.7: $\quad$ Execution time of 3D anisotropic diffusion filtering and 3D median

filtering. 75

Table 3.8: $\quad$ Performance comparison of the 3D anisotropic diffusion filtering

kernel. 76

Table 3.9: $\quad$ Performance comparison of the 3D median filtering kernel... 78

Table 4.1: Configurations of LUT-based entropy calculation module that were considered in the presented architecture. 106 
Table 4.2: Operational workflow for performing volume subdivision-based

deformable image registration using the presented architecture

Table 4.3: Comparison of mutual information calculation time for subvolumes at various levels in volume subdivision-based deformable registration

algorithm

Table 4.4: Execution time of deformable image registration. 116

Table 4.5: $\quad$ Performance comparison of the presented FPGA-based implementation of intensity-based deformable image registration with an equivalent software implementation and prior approaches for acceleration of intensity-based registration.

Table 5.1: Design variables for FPGA-based architecture. Integer wordlengths are determined based on application-specific range information, and fractional wordlengths are used as parameters in the multiobjective optimization framework 136

Table 5.2: $\quad$ Number of solutions explored by search methods............................. 142

Table 5.3: $\quad$ Parameters used for the EA-based search........................................... 143

Table 5.4: Validation of the objective function models using post-synthesis results. The wordlengths in a design configuration correspond to the FWLs of the design variables identified earlier. 151

Table 6.1: Execution time for deformable image registration using low-dose CT. 167

Table 6.2: $\quad$ Execution time for deformable image registration using intraprocedural CT and preprocedural PET images 176 
Table 6.3: Interexpert variability in landmark identification across 20 image pairs. $\mathrm{PET}_{\mathrm{ALGO}}$ corresponds to the software implementation of the

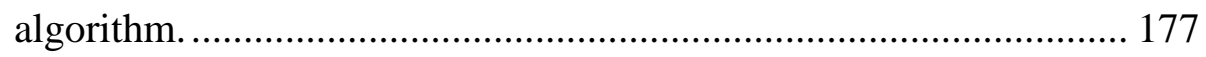

Table 6.4: Interexpert variability in landmark identification across 20 image pairs. PET $_{\mathrm{ALGO}}$ corresponds to the FPGA-based implementation of the

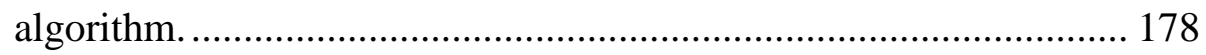




\section{List of Figures}

Figure 1.1: A typical IGI workflow and the scope of this dissertation work .......... 3

Figure 2.1: $\quad$ Two examples of pre- and intraprocedural image pairs. The arrows

indicate the targets that are visible in preprocedural images but not

visible in intraprocedural images. .............................................. 11

Figure 2.2: $\quad$ An example of volumetric image guidance using intraprocedural multislice CT and preprocedural MR.

Figure 2.3: Flowchart of image similarity-based image registration.

Figure 2.4: $\quad$ Example of preprocessing techniques employed prior to intensity-

based image registration.

Figure 2.5: Pareto front in the context of multiobjective optimization................. 36

Figure 3.1: A median filtering example using majority voting technique. ........... 52

Figure 3.2: Block diagram of the FPGA-based real-time 3D image preprocessing

system. 53

Figure 3.3: Typical voxel access pattern for neighborhood operations-based image processing. 54

Figure 3.4: Block diagram showing the input image memory and the input buffer configuration. 56

Figure 3.5: Pictorial representation of the notation used in the brick-caching

scheme. 57

Figure 3.6: Top-level block diagram of 3D anisotropic diffusion filtering. This

diagram indicates paths that are executed in parallel. 
Figure 3.7: $\quad$ Block diagram of the embedded Gaussian filter bank (for $\mathrm{N}=7$, corresponding Gaussian kernel size is 5).

Figure 3.8: $\quad$ Pipelined implementation of an individual Gaussian filter element

(Gaussian kernel size $=5)$.

Figure 3.9: A single stage (processing element) of the linear systolic median

filtering kernel 65

Figure 3.10: Linear systolic array architecture for median filter kernel using majority voting technique. 68

Figure 4.1: $\quad$ Pictorial representation of hierarchical volume subdivision-based deformable image registration and associated notation.

Figure 4.2: Pictorial representation of the acceleration approach. 86

Figure 4.3: $\quad$ Top-level block diagram of the architecture for accelerated implementation of deformable image registration. 88

Figure 4.4: Functional block diagram of voxel counter. 89

Figure 4.5: Functional block diagram of coordinate transformation unit. 91

Figure 4.6: Fundamentals of interpolation schemes. 92

Figure 4.7: Functional block diagram of partial volume interpolation unit. 94

Figure 4.8: Voxel access patterns of the reference and floating images encountered during image registration. 95

Figure 4.9: Organization of the reference image memory. 97

Figure 4.10: Organization of the floating image memory 98

Figure 4.11: Pipelined implementation of $\mathrm{MH}$ accumulation using dual port memory. 100 
Figure 4.12: $\quad$ Preaccumulate buffers to eliminate RAW hazards in $\mathrm{MH}$ accumulation pipeline 102

Figure 4.13: A flow diagram of steps involved in calculating $\mathrm{MH}_{\text {Rest }}$. 103

Figure 4.14: Error in entropy calculation corresponding to the two configurations of the multiple LUT-based implementation.

Figure 4.15: Qualitative validation of deformable registration between iCT and preCT images performed using the presented FPGA-based solution.

Figure 4.16: Qualitative validation of deformable registration between iCT and PET images performed using the presented FPGA-based solution.

Figure 5.1: $\quad$ Examples of parameterized architectural design style. 133

Figure 5.2: $\quad$ Framework for multiobjective optimization of FPGA-based image registration.

Figure 5.3: Comparison of the area values predicted by the adopted area models with those obtained after physical synthesis. 141

Figure 5.4: $\quad$ Pareto-optimized solutions identified by various search methods.... 144

Figure 5.5: Qualitative comparison of solutions found by partial search and EAbased search. 145

Figure 5.6: Quantitative comparison of search methods using the ratio of nondominated individuals (RNI). 147

Figure 5.7: Quantitative comparison of search methods using cover rate. 148

Figure 5.8: Relationship between MI calculation error and resulting image registration error. 
Figure 5.9: Results of image registration performed using the high-speed, FPGAbased implementation for design configurations offering various registration errors. 153

Figure 6.1: Integration of deformable registration into IGI workflow................. 156

Figure 6.2: Important steps for evaluating registration accuracy with low-dose CT.

Figure 6.3: Low-dose CT images generated by the dose-simulator...................... 160

Figure 6.4: Comparison of techniques for preprocessing low-dose CT images.. 162

Figure 6.5: Qualitative comparison of registration accuracy with low-dose CT. 165

Figure 6.6: $\quad$ Average registration error with respect to dose using software and FPGA-based implementations. 166

Figure 6.7: Graphic illustration of the quantitative validation approach used in the context of deformable registration between intraprocedural and preprocedural PET.

Figure 6.8: Registration of intraprocedural CT and preprocedural PET images using the FPGA-based implementation of deformable image registration. 176 


\section{Chapter 1: Introduction}

\subsection{Overview}

Image-guided interventions (IGIs), including surgeries, biopsies, and therapies, have the potential to improve patient care by enabling new and faster procedures, minimizing unintended damage to healthy tissue, improving the effectiveness of the procedures, producing fewer complications, and allowing for clinical intervention at a distance. As a result, IGIs has been identified by clinical experts to have a significant impact on the future of clinical care [1]. With further invention and development of imaging and image processing techniques, innovative minimally invasive image-guided inventions will replace conventional open and invasive techniques. Continuous three dimensional (3D) imaging and visualization for intraprocedural navigation, critically important to the success of IGI, has been technologically difficult until recently. However, the advances in medical imaging technology and visualization capabilities, leading to improved imaging speed and coverage, have prompted developments in imaging protocols and enabled volumetric image-guided procedures.

The efficiency and efficacy of IGIs is critically dependant on accurate and precise target identification and localization. Lack of clear target delineation could lead to lengthy procedures, larger than necessary safety margins and unintended

damage to healthy tissue-factors that undermine the very motivation behind IGIs. Intraprocedural imaging techniques provide a rich source of accurate spatial information that is crucial for navigation but often suffer from poor signal-to-noise 
ratio (SNR) and poor target definition from background healthy and/or benign tissue. As in most clinical protocols, IGIs are preceded by one or more preprocedural images, containing additional information, such as contrast-enhanced structures or functional details such as metabolic activity, which are used for diagnosis, treatment/navigation planning, etc. Combining this functional and/or contrast information with intraprocedural morphological and spatial information, through coregistration between pre- and intraprocedural images, has been shown to improve the intraprocedural target delineation [2-6].

Achieving this registration between intraprocedural and preprocedural images is a fundamental need during the IGI workflow. Moreover, given the on-demand nature of IGIs, this alignment should be achieved sufficiently fast so as not to affect the clinical workflow. Earlier approaches to meet this need primarily employed rigid body approximation, which can be less accurate because of non-rigid tissue misalignment between these images. Intensity-based deformable registration is a promising option to correct for this misalignment. These algorithms are automatic, which is an important aspect that enables their easy integration into many applications; However, the long execution times of these algorithms have prevented their use in clinical workflow. In addition, since this technique is based on intensitybased alignment between images, it is sensitive to the SNR of the images to be registered. Consequently, the images (in particular, intraprocedural images that are characterized with poor SNR) need to be preprocessed and de-noised before they can be registered. This workflow for providing improved visualization during IGIs is illustrated in Figure 1.1. 


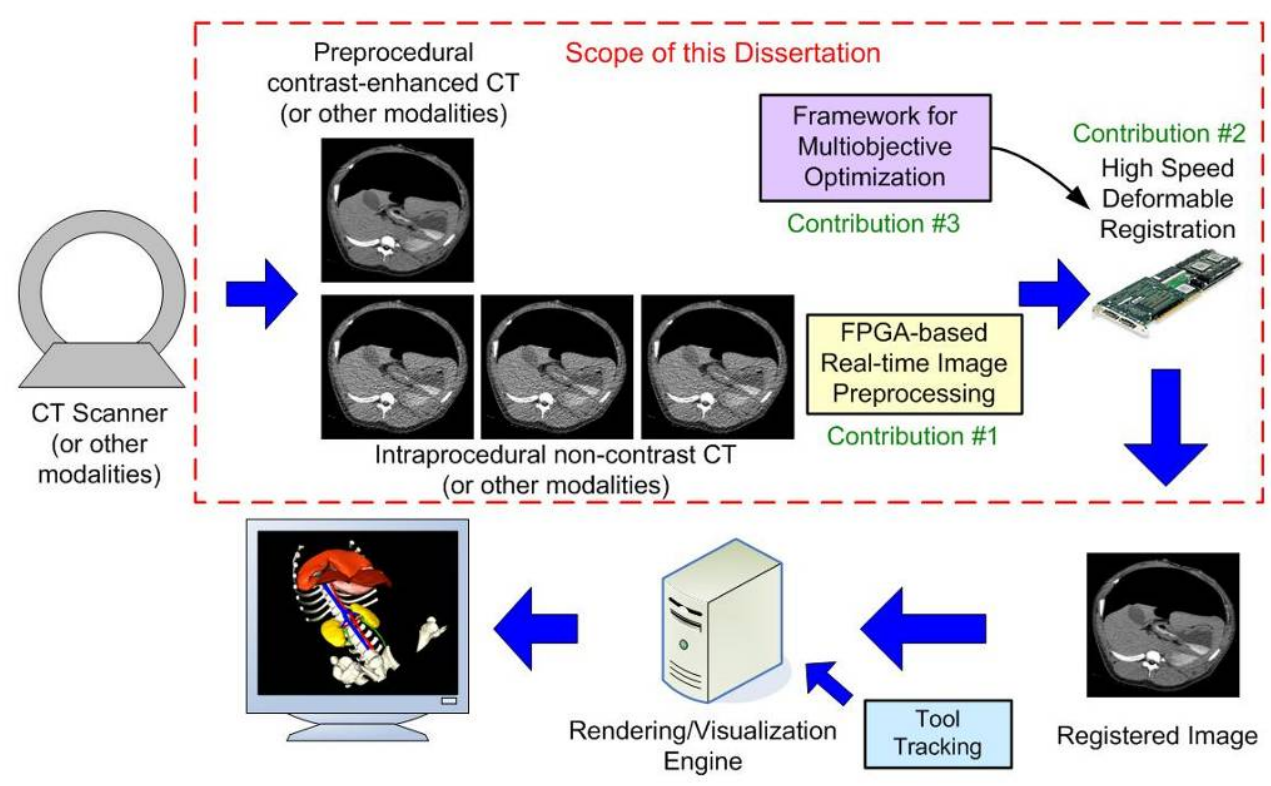

Figure 1.1: A typical IGI workflow and the scope of this dissertation work

The overall goal of this dissertation work is to improve the identification and localization of targets during image-guided interventions through automatic, fast, and accurate deformable image registration between preprocedural and intraprocedural images. With this accurate registration and fusion of complementary information an interventionist will be able to visualize accurately aligned anatomical structures (such as vasculature) and/or functional (metabolic) activity not natively present in the routine intraprocedural scans and thereby improving the targeting capability.

\subsection{Contributions of this Dissertation}

The specific goal of this dissertation work is to develop and validate the core components of this advanced image processing system, which will enable improved visualization and target-delineation during image-guided procedures. These core components are identified in Figure 1.1. First, we employ reconfigurable hardware platform to develop an architecture for real-time implementation of image 
preprocessing techniques commonly used in the context of IGI. Second, we develop an field-programmable gate array (FPGA)-based architecture for accelerated implementation of intensity-based deformable image registration. Third, we propose a multiobjective optimization framework to analyze conflicting tradeoffs between accuracy and hardware complexity of finite precision implementations of signal processing application, such as presented in this work. Finally, we demonstrate the feasibility of developing novel IGI applications leveraging the aforementioned components.

In the following sections we elaborate further on the main contributions of this dissertation.

\subsubsection{Real-time 3D Image Preprocessing}

Image preprocessing, which consists of filtering and de-noising, is a prerequisite step in many image processing applications. Especially in the context of IGI, where intraprocedural images are characterized by poor signal-to-noise ratio, image preprocessing is required prior to advanced image analysis operations such as registration, segmentation, and volume rendering. Moreover, the interactive nature of IGIs necessitates equivalent image processing speed so that these operation can be performed in a streamlined manner without any additional processing latency.

Most reported techniques for accelerated implementation of image processing algorithms have primarily focused on one-dimensional (1D) or two-dimensional (2D) cases [7-11]. These techniques do not adequately address the need for accelerating these operations in $3 \mathrm{D}$, which is required for providing volumetric image-guidance during minimally invasive procedures. Furthermore, some of the earlier techniques 
used for acceleration cannot be extended to 3D, whereas for some others the 3D extension is nontrivial.

This dissertation presents an FPGA-based novel architecture for accelerated implementation of common image preprocessing operations. This architecture is reconfigurable and supports multiple filtering kernels such as 3D median filtering, and 3D anisotropic diffusion filtering within the same framework. The architecture presented in this work is faster than earlier reported techniques, supports larger kernel dimensions, and is capable of meeting the real-time data processing need of most IGIs. Although developed in the context of IGIs, this architecture is general-purpose and can be applied to meet preprocessing needs of many medical as well as nonmedical applications.

\subsubsection{Hardware-Accelerated Deformable Image Registration}

Image registration between preprocedural images (acquired for diagnosis and treatment planning) and intraprocedural images (acquired for up-to-date spatial information) is an inherent need in the IGI workflow. Accurate and fast registration between these images will enable the fusion of complementary information from these two image categories and can enable improved treatment site identification and localization and navigation during the procedure.

Several fiducial or point-based, mechanical alignment-based and intensitybased rigid alignment techniques [12-16] have been proposed for this purpose. Some of these techniques are not automatic and almost all of them employ the rigid body approximation, which is often not valid due to tissue deformation between these two image pairs. Deformable image registration techniques can compensate for both local 
deformation and large-scale tissue motion and are the ideal solution for achieving the aforementioned image registration. Some studies, in particular, have independently underlined the importance of deformable image registration for IGIs [17-19]. However, despite their advantages, deformable image registration algorithms are seldom used in current clinical practice due to their computational complexity and associated long execution times (which can be up to several hours).

This dissertation presents a novel FPGA-based architecutre for accelerated implementation of a proven automatic and deformable image registration algorithm, specially geared toward improving target delineation during image-guided interventions. This architecture accelerates calculation of image similarity, a necessary and the most time consuming step in image registration, by greater than an order of magnitude and thereby reducing the time required for deformable registration time from hours to minutes. This design is tuned to offer registration accuracy comparable to that achievable using software implementation. Furthermore, we validate this high-speed design and demonstrate its feasibility in the context of clinical applications such as computed tomography (CT)-guided interventional applications. This accuracy, coupled with the speed and automatic nature of this approach represents a first significant step toward assimilation of deformable registration in the IGI workflow.

\subsubsection{Framework for Optimization of Finite Precision Implementations}

An emerging trend in image processing, and medical image processing, in particular, is custom hardware implementation of computationally intensive algorithms for achieving high-speed performance. The work presented in this 
dissertation has a similar spirit in the context of advanced image processing required during IGIs. For reasons of area-efficiency and performance, these implementations often employ finite-precision datapaths. Identifying effective wordlengths for these datapaths while accounting for tradeoffs between design complexity and accuracy is a critical and time consuming aspect of this design process. Having access to optimized tradeoff curves can equip designers to adapt their designs to different performance requirements and target specific devices while reducing design time.

This dissertation proposes a multiobjective optimization framework developed in the context of FPGA-based implementation of medical image registration. Within this framework, we compare several search methods and demonstrate the applicability of an evolutionary algorithm-based search for efficiently identifying superior multiobjective tradeoff curves. In comparison with some earlier reported techniques, this framework allows non-linear objective functions, multiple fractional precisions, supports a variety of search methods, and thereby captures more comprehensively the complexity of the underlying multiobjective optimization problem. We also demonstrate the applicability of this framework for the image registration application through synthesis and validation results using Altera Stratix II FPGAs. This strategy can easily be adapted to a wide range of signal processing applications, including areas of image and video processing beyond the medical domain.

\subsection{Outline of this Dissertation}

The rest of the dissertation is organized as follows: Chapter 2 provides background on image-guided interventions, image preprocessing, and image 
registration; and presents related work in the context of the contributions of this dissertation. In Chapter 3, FPGA-based architecture for real-time implementation of 3D image processing techniques such as median filtering and anisotropic diffusion filtering are presented. Chapter 4 deals with deformable image registration. We outline the intensity-based deformable image registration algorithm and present a novel architecture for accelerated implementation of this algorithm. In Chapter 5, a framework for multiobjective optimization of limited precision implementations of signal processing algorithms is presented. Chapter 6 introduces some novel imageguided procedures and demonstrates the feasibility of our approach in the context of these applications. Finally, in Chapter 7 conclusions and future work are presented. 


\section{Chapter 2: Background and Related Work}

\subsection{Image-Guided Interventions}

IGIs began to emerge in the last quarter of the 20th century, picked up pace in the 1990s, and may become routine in the 21st century. Minimal invasiveness is the defining characteristics of these procedures. This feature can lead to less patient morbidity, time and cost efficient procedures, faster recovery and improve the procedure outcomes. During these procedures, the internal anatomy is accessed through a single or few small holes on the patient's skin rather than though large incisions. The interventionist introduces the appropriate tool (electrode or biopsy needle, or/and endoscope) through this port and tries to navigate his/her way to the target (typically a malignant spot) in order to deliver a localized treatment or take out a sample for further investigation. Now, because the access to the internal anatomy is through a single port, the only way to visualize the location, orientation and the path of approach of the tool is by using external imaging techniques (that is there is no direct visual feedback).

Any intraprocedural imaging technique used must be near real-time and thus allow tracking underlying anatomy and flexible instruments and catheters as and when required (“on-demand” performance) during the procedure. 2D Ultrasound (US) and CT fluoroscopy have been conventionally used to guide placement of biopsy needles and therapy delivery devices during IGIs [18, 20, 21]. However, technological improvements such as multi-slice CT scanners, interventional MR, 3D ultrasound (US), isocentric C-arms and other advanced imaging systems have enabled 
the application of IGI to clinical domains such as interventional radiology, neurosurgery, orthopedics, ENT surgery, cranio- and maxillofacial surgery and other surgical specialties [22-24]. For example, Philips medical systems, one of the leading medical imaging equipment manufacturers, has announced a 256-slice CT scanner [25] which provides higher imaging speed (up to 8 volumes/s) and coverage $(8 \mathrm{~cm})$ is ideally suited for performing CT-guided procedures. Availability of easy access MR scanners, such as open-configuration MR scanner from GE Healthcare [26] along with its improved imaging speed has enabled development of MR-guided procedures. Image quality and acquisition speed of 3D ultrasound have also been enhanced through use of latest transducer technology and digital reconstruction and it can now be used for providing image-guidance during procedures. Moreover, real-time volumetric visualization capabilities, that enable interactive display of images during the procedure, are also now available [27, 28]. As a result, an emerging trend in IGI workflow is to use volumetric imaging modalities for providing real-time intraprocedural guidance. This dissertation, therefore, focuses on 3D image processing and registration in the context of IGIs.

\subsubsection{Role of Preprocedural Imaging}

Intraprocedural imaging techniques provide (or, are a rich source of) accurate spatial information which is crucial for navigation but offer poor target identification from the background healthy and/or benign tissue (see Figure 2.1). Most imageguided procedures are preceded by a preprocedural image which is used for diagnosis, treatment/navigation planning, etc. These preprocedural images are primarily acquired under different (often slow) imaging protocol and typically contain 
additional information, such as contrast-enhanced structures or functional information such as metabolic activity which is used for diagnosis and tissue differentiation prior to the treatment. Figure 2.1(a) shows contrast-enhanced structures in a preprocedural image which are not clearly visible in intraprocedural images. Figure 2.1(b) illustrates the metabolic activity shown in the PET scans which can be used to identify cancerous tumors. Availability of this functional and contrast information from the preprocedural images can be used to augment the purely morphological and spatial information from the intraprocedural images which will greatly improve the intraprocedural target delineation [2-6, 29]. Therefore, there is a clear need to combine this complementary information from the pre and intraprocedural images to facilitate this task.
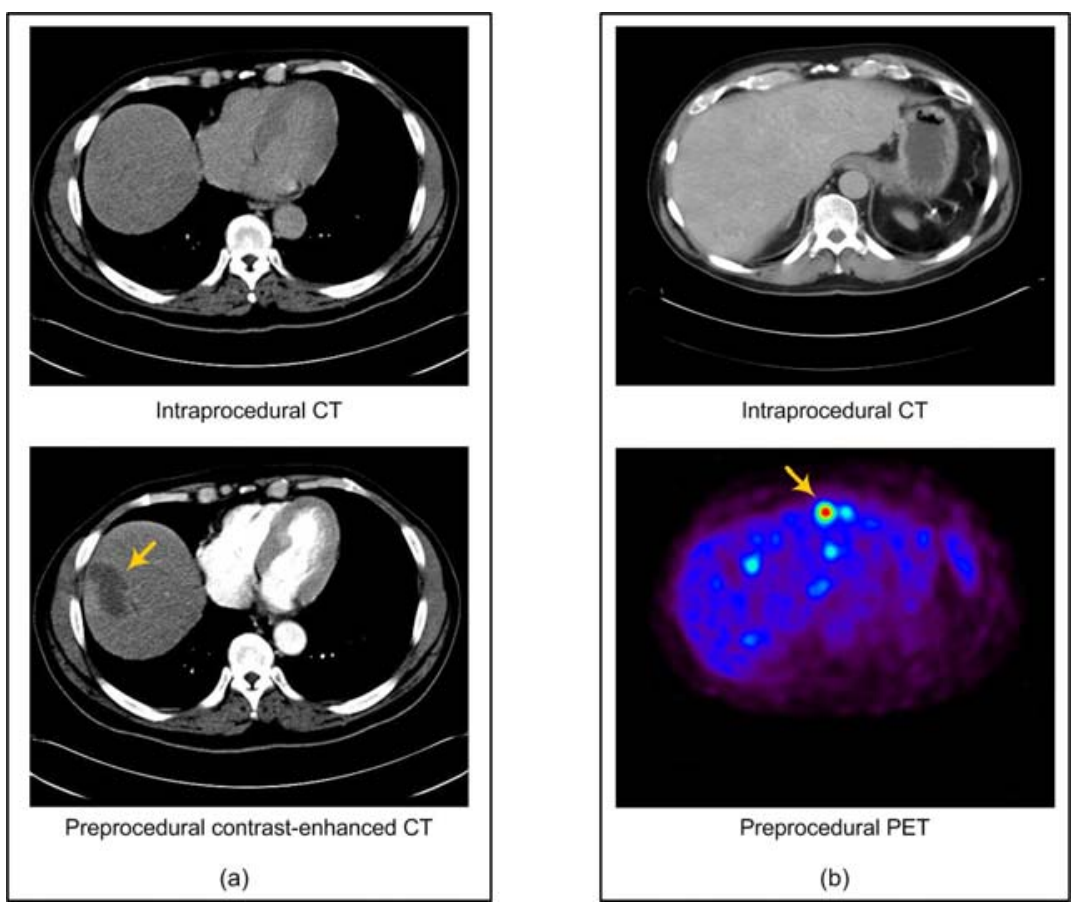

Figure 2.1: Two examples of pre- and intraprocedural image pairs. The arrows indicate the targets that are visible in preprocedural images but not visible in intraprocedural images. 


\subsubsection{Need for Image Registration}

Aligning or registering the intraprocedural images with the preprocedural image is a fundamental need in the IGI workflow. In fact, image registration has been identified as an enabling technology for image-guided surgical and therapeutic applications [30]. Figure 2.2 shows an example of volumetric image-guidance using image registration between volumetric CT and magnetic resonance imaging (MRI) scans for a neurosurgical application. There are, however, many technological and logistic challenges in achieving this image registration. First, the intra- and preprocedural images to be registered are acquired at different times and using different scanners. As a result, there is invariably misalignment of anatomical structures between these two images. This misalignment is caused because of the

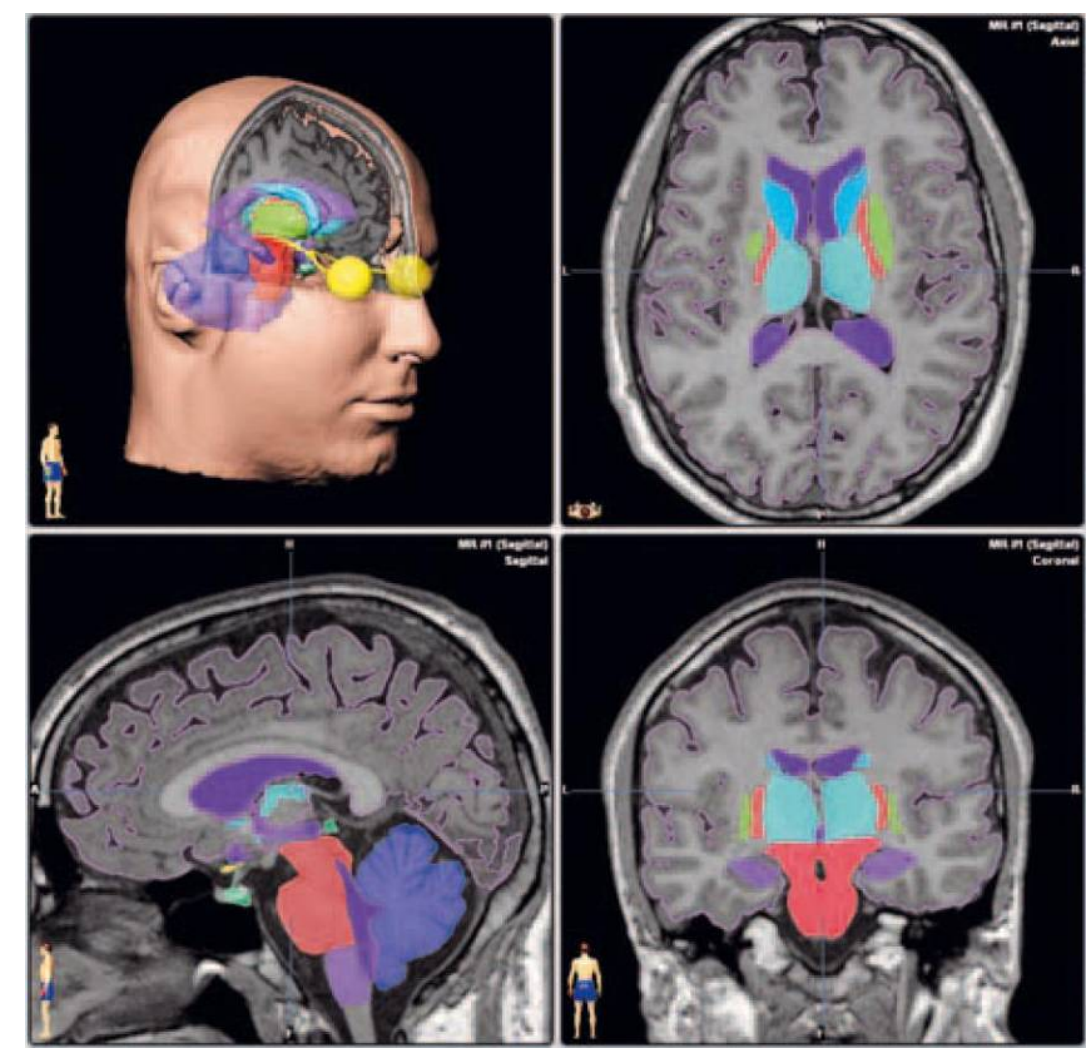

Figure 2.2: An example of volumetric image guidance using intraprocedural multislice CT and preprocedural MR. 
systemic offsets in scanner coordinate systems and due to non-rigid anatomical changes arising from pose and diurnal variations at the time of image acquisition. Second, the images to be combined can be of two completely different modalities (such as PET and CT). Furthermore, given the on-demand nature of IGI applications this registration should be achieved in a reasonably fast time. In summary, accurate, multi-modal, and fast image registration is essential for IGIs [17, 31]. The following section provides an overview of image registration.

\subsection{Classification of Image Registration}

Medical image registration is the process of aligning two images that represent the same anatomy at different times, from different viewing angles, or using different imaging modalities. Image registration is an active area of research and over the last several decades there have been numerous publications outlining various methodologies to perform image registration and its applications. Maintz and Viergever [32] and Hill et al. [33] have presented a comprehensive summaries of the entire gamut of the image registration domain. In general, image registration can be classified based on image dimensionality, nature of registration basis, nature of transformation models, type of modalities involved, etc. From the context of IGI,

Table 2.1: $\quad$ Broad classification of image registration in the context of IGI.

\begin{tabular}{cccccc}
\hline $\begin{array}{c}\text { Registration } \\
\text { Basis }\end{array}$ & $\begin{array}{c}\text { Method } \\
\text { based on }\end{array}$ & Retrospective & Automatic & Deformable & $\begin{array}{c}\text { Compute } \\
\text { Intensive }\end{array}$ \\
\hline $\begin{array}{c}\text { Extrinsic } \\
\text { Information }\end{array}$ & Fiducial & $\mathrm{N}$ & $\mathrm{Y}$ & $\mathrm{N}$ & $\mathrm{N}$ \\
\cline { 2 - 6 } & Stereotactic & $\mathrm{N}$ & $\mathrm{Y}$ & $\mathrm{N}$ & $\mathrm{N}$ \\
\hline \multirow{2}{*}{$\begin{array}{c}\text { Intrinsic } \\
\text { Information }\end{array}$} & \begin{tabular}{c} 
Landmark \\
\cline { 2 - 5 }
\end{tabular} & $\mathrm{Y}$ & $\mathrm{N}$ & $\mathrm{Y}$ & $\mathrm{N}$ \\
\cline { 2 - 6 } & Or Surface & $\mathrm{Y}$ & $\mathrm{N}$ & $\mathrm{Y}$ & $\mathrm{N}$ \\
\cline { 2 - 6 } & Intensity & $\mathrm{Y}$ & $\mathrm{Y}$ & $\mathrm{Y}$ & $\mathrm{Y}$ \\
\hline \hline
\end{tabular}


however, we broadly classify image registration into two main approaches. First, techniques based on extrinsic information and second, techniques based on information that is intrinsic to the image. We briefly describe these two techniques and outline some popular image registration methods in each category. A summary of this classification is also presented in Table 2.1.

\subsubsection{Image Registration using Extrinsic Information}

Methods based on extrinsic information rely on information that is not natively a part of the medical image. This includes artificial external objects that may be attached to the patient and are within the field of view of the image. These objects are designed such that they are clearly visible and accurately detectable in all of the pertinent modalities that are to be registered. As a result, the registration of the acquired images is usually easy, fast, and can be automated with relative ease. In addition, because the registration involves simply establishing correspondence between external objects, it can be achieved explicitly without a need for complex optimization techniques. One major limitation of these methods, however, is that they are not retrospective. This means that advanced planning is required and provisions must be made at the time of preprocedural imaging for that image to be used at a later point. Furthermore, due to the nature of the registration these methods are mostly limited to rigid transformation model only.

Stereotactic frame is another commonly used external object. There are many reported image registration applications, especially in the context of neurosurgery, that employ a stereotactic frame to establish spatial correspondence between images [34, 35]. These methods employ a frame screwed rigidly to the patient's skull that is 
usually fitted with imaging markers that are visible in imaging modalities such as CT, MRI, and X-ray. Visibility of these markers in both pre- and intraprocedural images will then allow registration of these images using a least-square based alignment technique. These techniques have been shown to be relatively accurate for rigid anatomy such as the brain [36], but are relatively more invasive. Less invasive techniques using markers attached to the skin have also been reported [37], but they tend to offer less accurate image registration because skin can move. More recently, there have also been efforts toward developing systems based on optical tracking methods that will allow frameless stereotaxy [38]. Despite these advances, these methods are fundamentally limited to providing only rigid alignment between a pair of images.

\subsubsection{Image Registration using Intrinsic Information}

These methods are based on intrinsic properties and contents of patientgenerated images. Registration may be based on a limited set of identified salient points (landmarks), on the alignment of segmented anatomical structures (segmentation or feature based) such as organ surfaces or directly based on the image intensity values (voxel property based).

Landmark-based registration [35, 39, 40] involves identification of the locations of corresponding points within different images and determination of the spatial transformation with these paired points. These landmarks are usually identified by a user in an interactive fashion. Landmark-based methods are often used to find rigid or affine transformations. However, if the sets of points are large enough, they may be used for more complex non-rigid transformations as well. Registration 
methods based on landmark identification can be retrospective, but they are not fully automatic becasue they require user interaction.

Segmentation-based image registration methods are based on extracting matching features and organ surfaces from the two images to be registered. These features and organ surfaces are then used as the only input for the alignment procedures. The alignment between the features/surfaces can be either based on rigid transformation models or achieved using deformable mapping. The rigid modelbased approaches are more popular and a 'head-hat' registration method based on this approach has been successfully applied to the registration of multimodal images such as PET, CT, and MR [41-43]. Popular segmentation-based techniques that involve deformable mapping of surfaces, such as the ones based on snakes or active contour models, have been shown to be effective in intersubject and atlas registration, as well as for registration of a template to a mathematically defined anatomical model [44, 45]. Segmentation-based techniques are retrospective, support multi-modal registration, and are computationally efficient. However, the accuracy of registration is dependant on the segmentation accuracy. Moreover, these methods are not fully automatic as the segmentation step is often performed semi-automatically.

Voxel property-based methods, which are based on image intensity values, are the most interesting methods in the current research. Theoretically, these are the most flexible of the registration methods since they use all of the available information throughout the registration process. In addition, these methods can be completely retrospective, fully automatic, allow multi-modal registration and generally are more accurate. The following section provides a detailed overview of intensity-based image 
registration. Although these methods have existed for a long time, their extensive use in clinical applications with 3D images has been limited because of associated computational costs. This dissertation work addresses this aspect through the use of hardware acceleration.

\subsection{Intensity-Based Image Registration}

Image registration that is based on voxel intensities is the most versatile, powerful, and inherently automatic way of achieving the alignment between two images. This method attempts to find the transformation $\hat{T}$ that optimally aligns a reference image RI, with coordinates $x, y$, and $z$, and a floating image FI under a image similarity measure $\mathbb{F}$. This process is summarized in the following equation and is represented pictorially in Figure 2.3.

$$
\hat{T}=\underset{T}{\arg \max } \mathbb{F}(R I(x, y, z), F I(T(x, y, z)))
$$

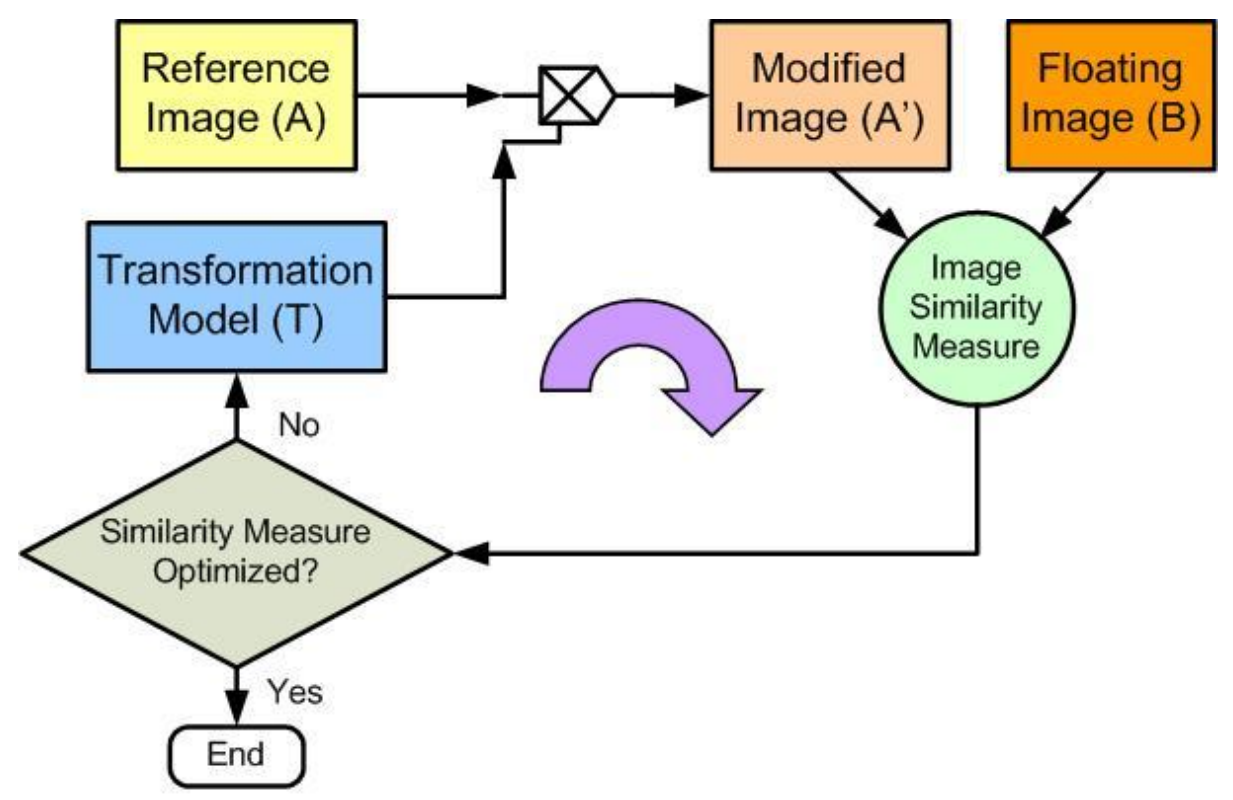

Figure 2.3: Flowchart of image similarity-based image registration. 
In the case of intensity based registration, the similarity measure $\mathbb{F}$, which provides a numerical value to indicate the degree of misalignment between the images, is completely based on voxel intensities in the reference and the floating images. Image transformation $T$ maps the reference image voxels into the floating image space. Depending on the transformation model employed, this mapping is either rigid, affine, or deformable. The optimization algorithm, on the other hand, searches for the best transformation parameters that optimally align the given two images. These three components form an integral part of intensity-based image registration and are described in the following sections.

\subsubsection{Transformation Models}

A transformation model provides a way to describe the misalignment between the reference and the floating images. The ability of image registration to accurately represent and recover this misalignment is fundamentally limited by the nature of the transformation model employed. For example, rigid transformation model typically offers inferior image registration accuracy as compared with computationally intensive, non-rigid transformation models, if the underlying misalignment is nonrigid. A comprehensive survey on image transformation models can be found in [46, 47]. The following subsections describe the transformations most commonly used in intensity-based image registration.

\subsubsection{Rigid and Affine Models}

Affine or linear registration is a combination of rotation, translation, scaling and shear parameters that map the reference image voxels into floating image space. 
Voxel scaling and shearing factors are constant for rigid registration, which is a special case of affine transformation, and as such are excluded from optimization process. Both these transformation models can be represented using a $4 \times 4$ transformation matrix. For example, a rigid transformation matrix $\mathrm{T}_{\text {global }}$ can be constructed as:

$$
T_{g l o b a l}=\left[\begin{array}{cccc}
r_{x x} & r_{x y} & r_{x z} & d_{x} \\
r_{y x} & r_{y y} & r_{y z} & d_{y} \\
r_{z x} & r_{z y} & r_{z z} & d_{z} \\
0 & 0 & 0 & 1
\end{array}\right],
$$

where $r_{i j}$ entries represents the components of the rotation matrix, while the $d_{i}$ entries represent the translation parameters. The coordinate transformation of a reference image voxel $\bar{v}_{r}$ into floating image space $\left(\bar{v}_{f}\right)$ can then simply be achieved through matrix multiplication:

$$
\bar{v}_{f}=T_{\text {global }} \cdot \bar{v}_{r} .
$$

Techniques based on rigid and affine transformation models have been successfully employed previously [47-49]. These techniques, however, offer limited degrees of freedom in the transformation model.

\subsubsection{Deformable Models}

The strength of the deformable transformation models comes from the large number of degrees of freedom they offer for representing the misalignment between images. This allows modeling of not only gross misalignment between the images, but also local deformations. As a result, image registration techniques based on deformable transformation models are inherently capable of correcting for local misalignments and therefore are more accurate. 
Methods based on physical models perform image transformation by considering a set of internal and external forces and obtaining the corresponding deformation by applying these forces to a given model based on differential equations. Some examples of physical models used for image transformation found in the literature are elastic body [50], viscous fluid [51] and incompressible flow (optical flow) [44]. Some methods based on finite element models have also been employed for image registration, which apply predefined physical models to represent deformation in the images $[52,53]$. The key idea is to divide the image into subsets, each with some defined physical properties. For example, a subset can be labeled as rigid, while some others can be labeled as fluid (elastic). During the transformation process, the shape of rigid tissues will not change, while the shape of fluid tissues will vary according to their corresponding properties such as viscosity. Image transformation techniques using physical models have been successfully applied to deformable image registration. However, most of these techniques involve solving partial differential equations and are particularly computationally complex.

Another popular method to represent deformable transformation model is to use mathematical basis functions. These transformation techniques use basis functions to define the correspondence between the original and the transformed image. The basis functions may be defined in either Fourier or Wavelet domain, and the deformation field is modeled using trigonometric or wavelet basis functions, respectively. Ashburner and Friston [54] have reported a method based on this approach. The deformation between the two images may also be modeled in the spatial domain using polynomials. Polynomial-based image transformation 
techniques use a global transformation function defined by a transformation matrix that contains the transformation coefficients and a polynomial vector that contains the components of the polynomial used to model the transformation. The simplest case of polynomial-based image transformation is the affine transformation, which uses firstdegree polynomials. By increasing the degree of the polynomials, it is possible to model complex non-rigid transformation as well. However, this method is seldom employed due to difficulty in modeling small local transformations and that higherdegree polynomials suffer from several artifacts [46]. These drawbacks are addressed by spline-based representation. Splines are inherently continuous and consist of piecewise-polynomial functions. Splines are a generalization of the polynomial-based approach to image transformation in the sense that a polynomial representation is a spline with just one segment. Using piecewise-polynomial functions allows modeling of local deformations accurately without using high-order polynomials. Two different spline families that have been used extensively in the literature to model 3D transformations are thin-plate splines and B-splines. Kim et al. [55] and Rohr et al. [56] have reported methods based on thin-plate splines to perform deformable image registration. However, one major drawback of thin-plate splines is that they have infinite support. This means that even small local changes are propagated throughout the entire image, an effect that is undesirable in medical image registration. In comparison, B-splines offer finite support. For this reason, B-splines are currently the preferred basis functions for modeling deformable transformations [57, 58]. A limitation with B-spline-based transformations is that they tend to fail at tracking rotation of local features. Moreover, algorithms based on B-splines tend to be 
computationally intensive due to additional complexity associated with B-spline interpolations.

More recently, some algorithms based on hierarchical image subdivision approaches have been reported [59, 60]. These algorithms achieve deformable registration through registering image subvolumes using a locally linear transformation and then applying quaternion-based interpolation to obtain the transformation field. Algorithms based on such transformation models allow the modeling of internal rotations better than the spline-based approaches. These algorithms are computationally efficient and yet are capable of recovering local deformations. The deformable registration algorithm considered in this dissertation is also based on hierarchical volume (3D image)-subdivision. This algorithm and the architecture for its accelerated implementation is describes in Chapter 4.

\subsubsection{Image Similarity Measures}

An important component of image registration is the metric that quantitatively determines how similar two images are. This metric can then be used to judge how well a pair of images is aligned and also to guide the optimization procedure during image registration. In the case of intensity-based image registration this metric, or image similarity measure, is computed using the voxel intensities of the images involved in registration. There are many reported intensity-based similarity measures. These can be broadly classified into measures using only image intensities (for example, mean of square difference of intensities), measures using spatial (or neighborhood) information (for example, pattern intensity or gradient-based measures) and measures based on information theory (mutual information). 
The following sections briefly describe some widely used similarity measures. We use the following notation for this description. The images to be aligned are reference image ( $R I)$ and the floating image (FI). A transformation $T$ is applied to the voxels of the reference image. An image similarity measure is calculated over the region of overlap ( $X_{0}$ ) between the RI and the FI and $\bar{x}$ represents the location of a voxel in RI. $N$ represents the number of RI voxel that belong to $X_{0}$. The notations $p_{R I}, p_{F I}$, and $p_{R I, F I}$ represent the individual probability distribution function (PDF) of RI, individual PDF of FI, and the mutual PDF of RI and FI, respectively.

\subsubsection{Sum of Squared Intensity Differences (SSD)}

One of the simplest ways to achieve image alignments is to minimize the intensity difference between the RI and FI. The sum of squared intensity differences (SSD) measure tries to achieve that. The SSD between the two images is defined as:

$$
S S D(R I, F I)=\sqrt{\frac{1}{N} \sum_{\bar{x} \in X_{0}}(R I(\bar{x})-F I(T(\bar{x})))^{2}} .
$$

As expected this measure will be minimized when two images are aligned well. However, this measure is limited to work with images with same intensity patterns, or in other words, for mono-modality image registration. Furthermore, Holden et al. [48] have shown this similarity measure to be error-prone in the presence of noise.

\subsubsection{Normalized Cross-Correlation (NCC)}

If the assumption that registered images differ only by Gaussian noise is replaced with a less restrictive one, namely that there is a linear relationship between the two images, then the optimum similarity measure is the normalized cross- 
correlation. Cross-correlation in both space and frequency domains has been used as a voxel similarity metric. Cross-correlation in the space domain is defined by:

$$
N C C(R I, F I)=\frac{1}{N^{2}} \frac{\sum_{\bar{x} \in X_{0}}(R I(\bar{x})-\overline{R I})(F I(T(\bar{x}))-\overline{F I})}{\sigma_{R I} \cdot \sigma_{F I}}
$$

where $\overline{R I}$ and $\overline{F I}$ are the mean intensities of the RI and FI respectively, whereas $\sigma_{R I}$ and $\sigma_{F I}$ represent the standard deviations of RI and FI, respectively:

$$
\begin{gathered}
\sigma_{R I}=\frac{1}{N} \sqrt{\sum_{\bar{x} \in X_{0}}(R I(\bar{x})-\overline{R I})^{2}}, \\
\sigma_{F I}=\frac{1}{N} \sqrt{\sum_{\bar{x} \in X_{0}}(F I(\bar{x})-\overline{F I})^{2}} .
\end{gathered}
$$

Computation of this similarity measure can be time consuming as it requires calculating the mean and the standard deviation as well as the cross-correlation coefficient for the entire 3D images. Because of this high computational cost of performing cross-correlation, spatial domain correlation is usually performed between a whole image and a small portion of the other image. Cross-correlation is an effective voxel similarity measure for images with low noise, but it high calculation requirements make it a poor choice for real-time applications. Furthermore, it may not yield optimal performance when applied to noisy images [46], such as ultrasound and low-dose CT.

\subsubsection{Mutual Information (MI)}

Mutual information is a popular image similarity metric based on information theory. The rationale behind this similarity measure is to consider image registration as the process of maximizing the amount of information common to RI and FI, or 
minimizing the amount of information present in the combined images. When the images are perfectly aligned, the corresponding structures from both images will overlap, minimizing the combined-information. The use of mutual information for image registration was introduced by Collignon et al. [61] and Viola and Wells [62]. The MI is defined by:

$$
M I(R I, F I)=h(R I)+h(F I)-h(R I, F I),
$$

where the individual and mutual entropies are calculated as:

$$
\begin{gathered}
h(R I)=-\sum p_{R I}(x) \cdot \ln \left(p_{R I}(x)\right), \\
h(F I)=-\sum p_{F I}(x) \cdot \ln \left(p_{F I}(x)\right), \\
h(R I, F I)=-\sum \sum p_{R I, F I}(x) \cdot \ln \left(p_{R I, F I}(x)\right) \cdot
\end{gathered}
$$

A comprehensive survey of MI-based registration was presented by Pluim et al. [47]. Mutual information is a very effective similarity measure for multimodal image registration because it can handle nonlinear information relations between data sets [63]. Holden et al. [48] have demonstrated that mutual information-based techniques are, in general, superior to other techniques for deformable image registration.

A broadly used variant of mutual information is called normalized mutual information. The advantage of this similarity measure over mutual information is its overlap-independence. It was introduced by Studholme et al. [64] as:

$$
N M I(R I, F I)=\frac{h(R I)+h(F I)}{h(R I, F I)}
$$

There are several other intensity-based similarity measures beyond the ones listed and described here. These include ratio of image uniformity, pattern intensity, entropy of the difference image, etc. Mutual information, in comparison, is versatile, 
inherently multimodal, and accurate; and hence has emerged as a popular choice for both rigid and deformable image registration. In particular, the deformable registration algorithm, being accelerated through custom hardware implementation in this dissertation work, is also based on MI. See Chapter 4, for additional details.

\subsubsection{Optimization Algorithms}

Optimization algorithms are used to navigate the search space of transformation parameters and to identify the optimal combination of transformation parameters that best aligns a pair of images. It must be noted, that most often the number of parameters to be searched for is more than one and this requires multidimensional optimization algorithms. Another desired feature of an optimization algorithm is that it requires fewer number of objective function evaluations. In the case of intensity-based image registration, the objective function to be optimized is the voxel similarity function. This calculation, usually, is compute intensive and hence faster convergence is ideal. We briefly summarize common multidimensional optimization scheme employed in the context of image registration.

The downhill simplex method, first introduced by Nelder and Mead [65] is an unconstrained nonlinear optimization technique. A simplex is a geometrical figure defined by $\mathrm{N}+1$ points in an $\mathrm{N}$-dimensional space. The simplex method starts by placing a regular simplex in the solution space and then moves its vertices gradually towards the optimum point through an iterative process. The downhill simplex algorithm searches for the optimum value through a series of geometrical operations on the simplex. Examples of these operations include reflection, reflection and expansion, contraction, multiple contractions etc. Shekhar et al. [49, 66] and Walimbe 
et al. [60,67] have reported successful use of this optimization technique for voxel similarity-based image registration.

Univariate optimization method tries to solve the multidimensional optimization problem by breaking it into multiple one-dimensional optimization problems. This is achieved by optimizing the variables, one variable at a time and then repeating this step until convergence. This method is simple, but can suffer from poor convergence in the presence of steep valleys in the search space. Powell's method builds upon the univariate method with an important distinction, that the search direction does not have to be parallel with any of the variable axes. Thus, it is possible to change multiple variables at the same time. This can achieve faster convergence and effectively eliminate the convergence problem of the univariate method. This algorithm has widely been used for optimizing intensity-based image registration $[47,68,69]$.

Optimization based on genetic algorithms is a technique that mimics the genetic processes of biological organisms. Over many generations, natural populations evolve according to the Darwinian principles of natural selection and the "survival of the fittest". Common operations involved in this method are crossover, mutation and fitness evaluation. By following this process, genetic algorithms are able to adapt starting solutions and ultimately find the optimal solution. These techniques are capable of efficiently searching a complex optimization space. However, representation of solutions in a genetic algorithm framework can be challenging and limit their effectiveness especially in the context of deformable 
registration (due to large number of parameters). Examples of genetic algorithmbased optimization for image registration can be found in [70, 71].

Optimization using simulated annealing techniques involves minimization methods based on the way crystals are generated when a liquid is frozen by slowly reducing its temperature [65]. These algorithms work distinctly from the techniques described earlier, in that they do not strictly follow the gradients of the similarity measure. Instead, they move randomly, depending on the "temperature" parameter. While the "temperature" is high, the algorithm allows greater variations in the variables to be optimized. As the "temperature" decreases, the algorithm further constrains the variation of the variables until a global optimum is reached. In general, simulated annealing techniques are more robust than earlier described methods. However, these techniques may require a large number of iterations to converge, especially in the presence of local minima. Some applications of simulated annealing techniques for image registration are described in [72, 73].

\subsection{Image Preprocessing}

As described in the previous section, intensity-based image registration (both rigid and deformable) utilizes similarity measures that are based on the voxel intensities of the images to be registered. As a consequence, these similarity measures are sensitive to quality of the images involved. Images with poor SNR can affect the calculation of a similarity measure and result into less-accurate image registration. While some similarity measures such as MI and NMI are less sensitive to noise, Holden et al. [48] have demonstrated that most intensity-based similarity measures 
(and resulting accuracy of image registration) are adversely affected in the presence of noise.

To address this aspect, several techniques for preprocessing images prior to image registration have been described. While some techniques focus on identifying a region or structures of interest in the images and exclude structures that may negatively influence the registration results [48, 49, 74], most preprocessing techniques rely on spatial-domain filtering operations on the images. Some reported techniques have employed low-pass filtering to remove speckle noise in ultrasound images, thresholding or filtering to remove noise, and blurring to correct for differences in the intrinsic resolution of the images [49, 64, 75]. All these spatial filtering techniques have shown to be effective in improving the image quality and the accuracy of image registration.

In the context of IGI, which is the primary application of the work presented in this dissertation, low-dose computed tomography (CT) and 3D ultrasound have emerged as the preferred intraprocedural volumetric imaging modalities. These modalities, although sufficiently fast for intraprocedural use, suffer from quantum noise and speckle noise respectively. Furthermore, due to presence of metallic tools

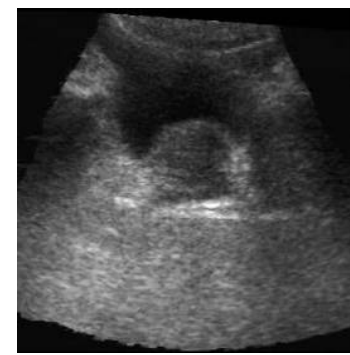

Ultrasound Image

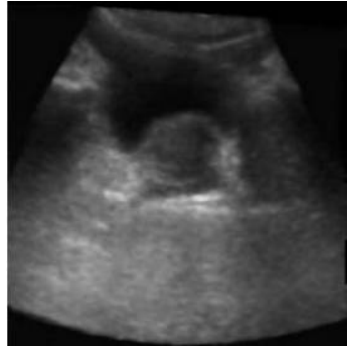

Filtered US Image Anisotropic Diffusion Filtering

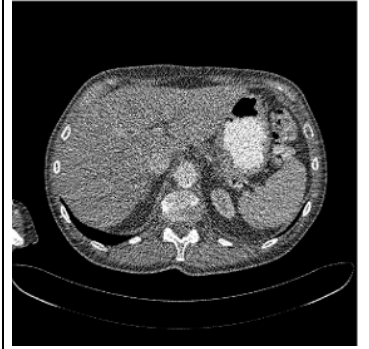

Low-dose CT Image

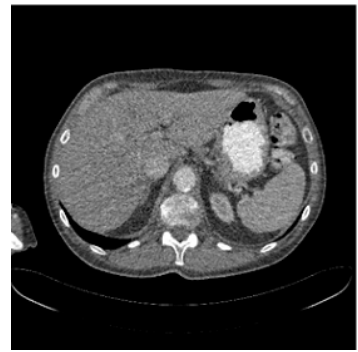

Filtered CT Image Median Filtering

Figure 2.4: Example of preprocessing techniques employed prior to intensitybased image registration. 
such as needles and catheters and associated photon scattering effects, intraprocedural CT images also suffer from metal artifacts. As a result, these images must be preprocessed and enhanced prior to registration with preprocedural images and subsequent visualization. Toward this end, anisotropic diffusion filtering and median filtering have been shown to be effective. Figure 2.4 shows an example application of these filtering operations. In particular, anisotropic diffusion filtering has been successfully applied for preprocessing of ultrasound, CT, and low-dose CT images [76-78]. Similarly, median filtering has been employed, both in spatial and sinogram domains, to reduce or eliminate metal artifacts and for filtering low-dose CT images $[79,80]$. In this dissertation we, therefore, focus on these two filtering techniques.

\subsubsection{Anisotropic Diffusion Filtering}

Anisotropic diffusion filtering is an iterative process which progressively smoothes an image while maintaining the significant edges. The nonlinear anisotropic diffusion algorithm for edge-preserving image smoothing was first proposed by Perona and Malik [81]. For a 3D image $I$ with intensities $I(\bar{v}, t)$, where $\bar{v}$ is a vector in the $3 \mathrm{D}$ space and $t$ is a given point in time (for the purposes of modeling the diffusion process), the diffusion process is described by the following equation:

$$
\frac{\partial I}{\partial t}=\operatorname{div}(c(\bar{v}, t) \cdot \nabla I(\bar{v}, t))
$$

where $c$ is the diffusion coefficient and takes a value between zero and 1 . In general, the diffusion coefficient is defined as a function of the image gradient (i.e., $c=f(|\nabla I|)$ ). For noisy images, Whitaker and Pizer [82] showed that gradient estimates taken from the image itself tend to be unreliable and proposed, instead, the 
use of a Gaussian-filtered version of the image to calculate the gradient values. Their proposed Gaussian filter has a standard deviation $\sigma(t)$ that decreases as the time ( $t$ ) increases, thus resulting in a multiscale approach. Dorati et al. [83] demonstrated the usefulness of Whitaker and Pizer's approach to 3D ultrasound image preprocessing. The diffusion coefficient that uses the Gaussian-filtered image (indicated as $G(\sigma(t))$ ) is then defined as:

$$
c=f(|\nabla G(\sigma(t)) \cdot I(\bar{v}, t)|) .
$$

Several diffusion functions have been proposed in the literature. The two most widely used are:

$$
c_{1}=\exp \left(-\left(\frac{|\nabla G(\sigma(t)) \cdot I(\bar{v}, t)|}{K}\right)^{2}\right),
$$

and

$$
c_{2}=\left(1+\left(\frac{|\nabla G(\sigma(t)) \cdot I(\bar{v}, t)|}{K}\right)^{1+\alpha}\right)^{-1} .
$$

These diffusion functions depend on the gradient of the Gaussian-filtered image, while the parameter $K$ adjusts the levels at which edges are diffused or preserved, to achieve the desired filtering effect.

\subsubsection{Median Filtering}

Median filtering is a nonlinear technique commonly used to eliminate speckle noise from ultrasound and impulse noise from other noisy images. This technique is called non-linear because it can not be represented as a direct convolution operation. 
This technique is based on rank-ordering of the intensity values present in an image. In addition, this filter is an edge-preserving filter. The advantage of this technique when compared to most linear (convolution-based) smoothing operators is that it smooths areas within a particular object, while preserving its edges. This feature is important, especially in the context of medical image registration, since it generally improves the accuracy of the image registration, segmentation, and visualization operations by preserving anatomical boundaries, while reducing random noise in the interiors of the structures. However, such filters, due to their nonlinear nature, tend to be computationally more intensive as compared with linear filtering operations.

The 3D realizations of these preprocessing operations, despite their effectiveness, can take several seconds when implemented in software. Consequently, for seamless integration into the IGI workflow these techniques must be accelerated so that their performance is comparable to the acquisition speed of intraprocedural images. This dissertation addresses this need through real-time implementation of 3D realizations of these operations as described in Chapter 3.

\subsection{Optimization of Finite Precision Implementations}

An emerging trend in image processing, and medical image processing, in particular, is custom hardware implementation of computationally intensive algorithms in the quest to achieve real-time performance. The work presented in this dissertation has a similar spirit in the context of advanced 3D image processing required during IGIs. For reasons of area (and power)-efficiency and performance, these implementations often employ limited-precision datapaths. In comparison, the original algorithms are often developed in software using the double-precision 
representation. Identifying effective wordlengths for these datapaths while accounting for tradeoffs between design complexity and accuracy is a critical and time consuming aspect of this hardware design process. This problem of converting floating-point implementations into fixed-point (or other limited precision representations) through identification of optimum wordlengths is an important problem in signal processing applications and has received considerable attention in the literature. Cantin et al. [84] and Todman et al. [85] provide a comprehensive review of techniques to identify optimal wordlengths. We briefly summarize some important approaches here.

\subsubsection{Optimal Wordlength Formulation}

Consider a system with $m$ internal variables and a wordlength $w_{i}$ associated with each variable. Further, each variable can take values between the lower $\left(w_{\text {min }_{i}}\right)$ and upper $\left(w_{\text {max }_{i}}\right)$ bound on the wordlength such that $w_{i} \in\left(w_{\text {mini }_{i}}, w_{\text {max }_{i}}\right)$. Each wordlength is an integer variable, and the wordlength configuration for the entire system can then be represented using a wordlength vector $W\left(W \in I^{m}\right)$ such as $\left\{w_{1}, w_{2}, \cdots, w_{m}\right\}$. Furthermore, $W \in\left(W_{\min }, W_{\max }\right)$; where $W_{\text {min }}=\left\{w_{\text {min }_{1}}, w_{\text {min }_{2}}, \cdots, w_{\text {min }_{m}}\right\}$ and $W_{\text {max }}=\left\{w_{\text {max }_{1}}, w_{\text {max }_{2}}, \cdots, w_{\text {max }_{m}}\right\}$. Consider a function $H$ associated with the system that defines the hardware implementation cost associated with a wordlength configuration $W$. Also, consider that the performance of this limited-precision, quantized system is characterized by function $p(W)$ and that the system must achieve a certain performance $P_{\min }$. The wordlength optimization problem can then be presented as: 


$$
\underset{W \in\left(W_{\min }, W_{\max }\right)}{\arg \min } H(W) \text {, such that } p(W) \geq P_{\min } \text {. }
$$

It must be noted that this formulation, for simplicity, considers only one objective function $H$ with respect to a predefined performance criterion $P_{\min }$. The more general multi-objective formulation is briefly described below and revisited in detail later in the context of FPGA-based implementation of deformable image registration (see Chapter 5).

\subsubsection{Simulation-Based Optimal Wordlength Search}

Optimal wordlength configuration that meets a certain performance criterion can be identified by solving analytical expressions, when the performance function $p$ can be represented analytically. Some earlier reported approaches have adopted this technique [86-90]. However, if the performance function can not be represented analytically, which is often the case for practical complex systems, simulation-based methods can be used to search for the optimal configuration. This involves searching the design space (defined by the wordlength vector ranges) and finding a solution that satisfies the design criteria. Some popular methods in this category are briefly described below. A detailed description of these methods can be found in [84, 91].

An exhaustive search attempts every possible combination of wordlengths between the predefined lower and upper bounds and evaluates the performance of each combination through simulation. The optimum wordlengths can then be selected from the simulation results. An exhaustive search is guaranteed to find the global optimal configuration, however, the number of solutions explored and the associated execution time increase exponentially as the number of variables increases. 
Another method, proposed by Sung and Kum [92] searches for the first solution that satisfies a given performance requirement or an error criterion. These method starts with an initial guess for the system configuration based on uni-variable simulations. The wordlength of each variable, as provided by this initial guess, is then sequentially incremented by one until a configuration that meets the error criterion is found. Although, this method is more efficient than the exhaustive search, finding the globally optimal configuration is not guaranteed.

A sequential search method $[84,93]$ that takes into account the performance sensitivity to determine the direction of the search is another way to approach this problem. This method starts with an initial guess based on uni-variable simulations; however, the further search direction is determined by the sensitivity of the performance to each variable. This sensitivity is estimated by calculating the gradient of the system performance with respect to all the variables and the search progresses in the direction of the variable (that is wordlength of that variable is incremented) that offers most improvement. It is also possible to consider hardware cost sensitivity instead of the performance sensitivity in this search method.

\subsubsection{Multiobjective Optimization}

One of the limitations of the optimization formulation described above is that search methods based on this formulation are limited to finding a single solution that satisfies a design objective. Most real-world problems (including the wordlength optimization problem), however, can have several objectives (that generally conflict with each other) that need to be achieved at the same time. For example, in the case of finite-precision implementations, hardware resource requirements and the 
implementation accuracy are two such conflicting objectives. Because of the conflicting nature of the involved objectives, multiobjective optimization problems do not normally have a single optimal solution and even necessitate a new definition of optimality.

The most commonly adopted notion in multiobjective optimization problems is that of Pareto optimality. A vector of decision variables $x^{*} \in \mathbb{F}$ is Pareto optimal if there does not exist another solution $x \in \mathbb{F}$ such that $f_{i}(x) \leq f_{i}\left(x^{*}\right)$, for all $i$, and $f_{j}(x)<f_{j}\left(x^{*}\right)$, for at least one $j$, where $f_{i}$ represents an objective function defined for every $x \in \mathbb{F}$. This definition of optimality almost always provides a set of solutions called the Pareto-optimal set. The set of vectors $x^{*}$ corresponding to the solutions in the Pareto-optimal set are called non-dominated solutions. This concept is pictorially illustrated in Figure 2.5.

Formulating the wordlength optimization as a multiobjective problem has merit because it allows finding a set of Pareto-optimal configurations representing strategically-chosen tradeoffs among the various objectives. This allows a designer to choose an efficient configuration that satisfies given design constraints and provides ease and flexibility in modifying the design configuration as the constraints change.

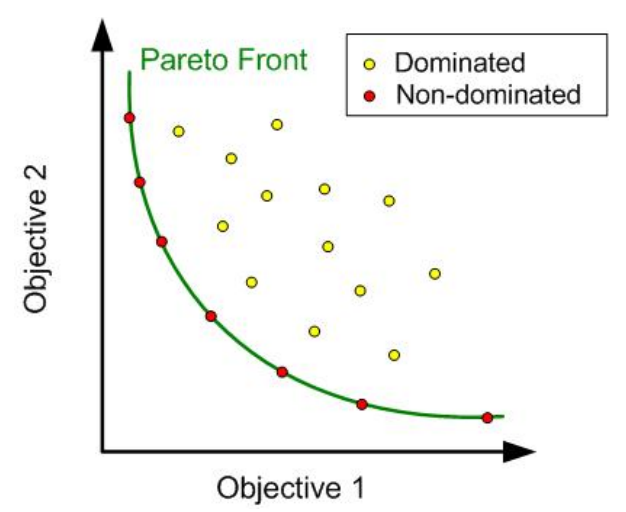

Figure 2.5: Pareto front in the context of multiobjective optimization. 
For example, Leban and Tasic [94] used error, delay, and area as objectives. Han and Evans [91] performed optimization of area and error through linear aggregation, while Givargis et al. [95] considered power and execution performance trade-off for system-on-chip architecture through series of monobjective optimizations. There are also some heuristic techniques that take into account tradeoffs between hardware cost and implementation error and enable automatic conversion from floating-point to fixed-point representations [96]. In this dissertation work, we develop a framework for multiobjective optimization of finite precision implementations. This framework has been developed for optimization of the FPGA-based image registration and has been validated through post-synthesis evaluation.

\subsection{Related Work}

\subsubsection{Real-Time Image Preprocessing}

Image preprocessing plays a crucial role in image understanding based systems, video processing, and in the medical imaging domain. Over last two decades much work was done on implementing image processing components in hardware. A detailed description of various single instruction multiple data (SIMD) and multiple instruction multiple data (MIMD) architectures can be found in [97, 98]. With availability of variety of computing platforms such as digital signal processors (DSPs), graphics processing units (GPUs), and FPGAs some of the image processing algorithms have also been mapped to these platforms for achieving superior performance. Most reported techniques, however, focus on $1 \mathrm{D}$ or $2 \mathrm{D}$ realizations and do not adequately address the need for accelerating these operations in 3D. Moreover, 
because of the larger 3D neighborhoods $\left(\mathrm{N}^{3}\right.$ as opposed to $\mathrm{N}$ or $\mathrm{N}^{2}$ ), data input requirements are increased and the performance achieved for $1 \mathrm{D}$ or $2 \mathrm{D}$ realizations may not translate to their corresponding 3D implementations. This additional complexity, coupled with the sheer size of 3D images (typically 2-5 million voxels), makes achieving real-time performance extremely challenging. Consequently, only a few designs for high-speed implementation of 3D image preprocessing techniques have been reported in the literature. We focus on implementations of anisotropic diffusion and median filtering and summarize those efforts here.

Rumph et al. [10] implemented the 2D nonlinear diffusion process on a graphics hardware. The primary focus of this work was to achieve acceleration through parallelism and better memory bandwidth. Gijbels et al. [8], on the other hand, have reported a VLSI architecture based on linear array technique for implementation of iterative diffusion process. A similar VLSI-based approach was also reported recently for $1 \mathrm{D}$ nonlinear signal processing [11]. Accelerated implementations of 3D anisotropic diffusion filtering using computing clusters have also been reported. Bruhn et al. [99, 100] have reported an approach using a 256-node Myrinet cluster, whereas Tabik et al. [101] have explored multiple parallel programming paradigms built on message passing and shared-memory architectures. Both these techniques have yielded near-linear speedups.

Accelerated implementations of median filters based on searching, sorting, and bit-level methods have previously been reported in the literature. We particularly focus on bit-level methods because they are well suited to finding the median of large 3D neighborhoods in hardware. Bit-level methods for median filtering can be 
classified into the bit-serial sorting, bit-serial searching, threshold decomposition, and majority voting-based methods. Bit-serial sorting is performed using sorting networks such as the odd-even exchange network and reduced bubble sort network [102, 103]. Bit-serial searching [104], also called the radix method, involves a bit-bybit search to find the median. The threshold decomposition method [105] provides a modular and parallel design, but the hardware requirements grow exponentially with the number of bits used to represent images. Majority voting methods are based on determining bit-wise majority starting from the most significant bits (MSBs). Lee and Jen $[9,106]$ have described a novel binary majority gate that can determine the majority of binary input signals using an inverter circuit. A compact majority voting circuit using an adder array to count the number of $1 \mathrm{~s}$ and a threshold comparator to determine an individual bit of the median is described by Benkrid et al.[7]. Variations on this approach have been described in the literature [107-109]. Systolic array architectures for bit-level sorting networks have been shown to improve concurrency of the bit-serial sorting designs [102, 103, 110-113]. The median filter design presented in this work is a combination and 3D extension of bit-serial searching and majority voting approaches.

In this dissertation work, we introduce a novel FPGA-based architecture of 3D anisotropic diffusion filtering. In addition, this work develops an architecture for 3D median filtering kernel, which is faster than existing solutions and is capable of supporting higher 3D kernel sizes. Our solution is compact, easily deployable and is capable of processing the intraprocedural images faster than their acquisition speeds. 


\subsubsection{Acceleration of Image Registration}

Intensity-based automatic image registration is a key component of modern medical imaging. Fast and accurate image registration can enhance many diagnostic and interventional applications. However, this task is also computationally intensive due to dimensionality of the images involved and memory-bound nature of the operation. It is this aspect that has limited the integration of intensity-based image registration (and that of deformable nature, in particular) in clinical applications. To address this aspect many researches have independently attempted to accelerate intensity-based image registration. Classification of these acceleration attempts has been reported by Plishker et al. [114]. We briefly summarize these attempts here.

\subsubsection{Multi-Processor and Supercomputer-Based Approaches}

Image registration problem lends itself well for acceleration through parallel implementation. Inherent data-parallel nature (same operations to be performed on every voxel of an image) of these algorithms makes them readily amenable to parallelization. The majority of earlier reported attempts to accelerate intensity-based deformable registration have primarily employed a multiprocessor approach. Ourselin et al. [115] reported a parallel implementation of affine registration using a 10processor cluster that provided a 6-fold speedup. Stefanescu et al. [116] implemented Demons algorithm [44] on a similar cluster of 15 2-GHz Pentium CPUs and achieved an 11-fold speedup for non-rigid image registration between a pair of magnetic resonance images. Similarly, Ino et al. [117] have reported a fast implementation of MI-based deformable registration using a 128-processor cluster. Another acceleration approach has been to use supercomputers, which offer a high degree of parallelism. 
Warfield et al. [118] performed deformable registration on a Sun supercomputer in 15 sec. However, interactive segmentation of the brain surface in the intraprocedural MR images took several minutes. Moreover, this implementation was specific to brain MR images because of high surface correspondence. Rohlfing et al. [57] have reported a speedup of 40 for a splines-based deformable registration algorithm using a 64-processor shared-memory supercomputer (SGI Origin 3800). Although these solutions delivered high performance by virtue of parallelization, the speedup achieved per processor was less than unity. Moreover, these solutions may not be cost effective, and because of their size, are unlikely to be suitable for clinical deployment.

\subsubsection{Graphics Processor (GPU)-based Approaches}

The recent emergence of powerful graphics processors (GPUs) has enabled a new direction for accelerating computationally intensive applications. Modern GPUs offer an array of processing elements that can offer customized data parallel processing. Many high level languages, such as Cg, Brook, CUDA, are emerging to aid the task of programming GPUs. This has enabled the use of GPUs for many other applications such as image registration beyond graphics domain. Strzodka et al. [119] reported the first implementation of image registration using the graphics hardware. This implementation accelerated a gradient flow-based image registration using graphics hardware. However, it was limited to registration of 2D images only and offered only limited speedup. Kohn et al. [120] have reported another implementation of gradient-flow based image registration that supports 3D images. Although, this implementation offered moderate speedup for rigid registration, the performance achieved for 3D deformable image registration was poor. Plishker et al. [121] have 
employed GPUs for applying transformations to images during rigid registration. This implementation achieved 3-fold improvement in execution time over a CPU-based implementation. More recently, Vetter et al. [122] have reported acceleration of MIbased multimodal registration using graphics hardware. Although, this implementation achieved accuracy comparable to that achieved using a software implementation, the speedup achieved was only about 5-fold. In summary, these reported solutions demonstrate how this promising platform can be utilized for certain image registration techniques. However, the architecture of GPUs along with their lack of efficient scatter operation is not optimally suitable for operations such as accumulation which is a prerequisite (accumulation of the mutual histogram [MH]) for calculation of MI. As a result, GPU-based solutions, despite being compact and low-cost, may not provide substantial acceleration for calculation of MI, which is the most versatile and robust image similarity measure.

\subsubsection{Other Approaches}

Emerging multi-core processors are able to accelerate medical imaging applications by exploiting the parallelism available in their algorithms. Ohara et al. [123] have implemented an MI-based 3D rigid registration algorithm on the Cell Broadband Engine (CBE) processor, which has nine processor cores on a chip and has a 4-way SIMD unit for each core. By exploiting the highly parallel architecture and its high memory bandwidth, this implementation with two CBE processors can compute MI around 11-times faster than a sequential implementation. However, this implementation does not support deformable image registration. 
General purpose hardware languages and compilers for transforming highlevel descriptions into hardware are becoming increasingly popular. Streams-C, Handel-C, Mitrion-C are examples of such tools. These tools allow direct translation of code developed using high-level languages such as C, Java, or Matlab into efficient hardware implementations. Although, these techniques have provided considerable speedup for applications involving matrix operations, linear algebra, and search, their performance in complex applications requiring architectural insights has been limited. For example, Jiang et al. [124] have reported a method for acceleration of splinesbased deformable image registration using Handel-C. The converted design, when implemented using a Xilinx device, could achieve speedup a of only 3.2 when compared with an equivalent software implementation.

In comparison with the techniques mentioned above, this dissertation work presents a novel FPGA-based architecture for high-speed implementation of MIbased deformable 3D image registration. This architecture is capable of accelerating MI calculation by a factor of 40 using a single computing element. Consequently, the execution time for deformable image registration is reduced from hours to a few minutes. Furthermore, this implementation is accurate, automatic, compact, and completely retrospective.

\subsubsection{Optimization of Finite Precision Implementations}

With the need for real-time performance in signal processing applications an increasing trend is to accelerate computationally intensive algorithms using custom hardware implementation. The architectures presented in this dissertation, for accelerated implementation of image preprocessing and image registration, fall into 
the same category. A critical step in going to a custom hardware implementation is converting floating-point implementations to fixed-point realizations for performance reasons. This conversion process is an inherently multidimensional problem, as several conflicting objectives, such as area and error, have to be simultaneously minimized. By systematically deriving efficient tradeoff configurations, one can not only reduce the design time [125] but can also enable automated design synthesis [96, 126]. Furthermore, these tradeoff configurations will allow designers to identify optimized, high quality designs for reconfigurable computing applications. The work presented in this dissertation develops a framework for optimizing tradeoff relations between hardware cost and implementation error in the context of FPGA-based image registration.

Earlier approaches to optimizing wordlengths used analytical approaches for range and error estimation [86-90]. Some of these have used the error propagation method (e.g., see [89]), whereas others have employed models of worst-case error [87, 90]. Although, these approaches are faster and do not require simulation, formulating analytical models for complex objective functions, such as MI, is difficult. Statistical approaches have also been employed for optimizing wordlengths [127, 128]. These methods employ range and error monitoring for identifying appropriate wordlengths. These techniques do not require range or error models. However, they often need long execution times and are less accurate in determining effective wordlengths.

Some published methods search for optimum wordlengths using error or cost sensitivity information. These approaches are based on search algorithms such as 
"Local," "Preplanned," and "Max-1" search [84, 93]. However, for a given design scenario, these methods are limited to finding a single feasible solution, as opposed to a multiobjective tradeoff curve. In contrast, the techniques we present in this dissertation are capable of deriving efficient tradeoff curves across multiple objective functions.

Other heuristic techniques that take into account tradeoffs between hardware cost and implementation error and enable automatic conversion from floating-point to fixed-point representations are limited to software implementations only [96]. Also, some of the methods based on heuristics do not support different degrees of fractional precision for different internal variables [87]. In contrast, the framework presented in this dissertation allows multiple fractional precisions, supports a variety of search methods, and thereby captures more comprehensively the complexity of the underlying multiobjective optimization problem.

Other approaches to solve this multiobjective optimization problem have employed weighted combinations of multiple objectives and have reduced the problem to mono-objective optimization [91]. This approach, however, is prone to finding suboptimal solutions when the search space is nonconvex [129]. Some methods have also attempted to model this problem as a sequence of multiple monoobjective optimizations [95]. The underlying assumption in this approximation, however, is that the design parameters are completely independent, which is rarely the case in complex systems. Modeling this problem as an integer linear programming formulation has also been shown to be effective [86]. But this approach 
is limited to cases in which the objective functions can be represented or approximated as linear functions of design variables.

Evolutionary algorithms (EAs) have been shown to be effective in solving various kinds of multiobjective optimization problems [130, 131] but have not been extensively applied to finding optimal wordlength configurations. An exception is the work of [94], which employs mono-objective EAs. In contrast, our work demonstrates the applicability of EA-based search for finding superior Paretooptimized solutions in an efficient manner, even in the presence of a non-linear objective function, such as MI. Moreover, our optimization framework supports multiple search algorithms and objective function models; and can easily be extended to a wide range of other signal processing applications. This optimization framework, which is developed and validated in the context of FPGA-based 3D image registration, is described in detail in Chapter 5 . 


\section{Chapter 3: Real-time 3D Image Processing}

This chapter presents an FPGA-based architecture for real-time implementation of 3D image pre-processing techniques commonly employed in IGI. First, we outline the filtering algorithms that are being accelerated in this work. Next, we present the architecture for their real-time implementation. Finally, we describe the realization of this architecture, analyze the effects of finite precision implementation, and compare the performance of this implementation with earlier reported efforts.

\subsection{Motivation}

Real-time and high-quality three-dimensional (3D) intraprocedural visualization is a critical need for IGIs. Recent advances in computer and transducer technology have made high-speed 3D imaging possible with high resolution and acquisition speed. Notably, low-dose computed tomography (CT) and 3D ultrasound have emerged as the preferred volumetric imaging modalities during many imageguided procedures [18, 132-135]. The advent of multislice CT allows high-resolution and high-frame-rate volumetric imaging of the operative field. In the continuous volumetric mode, multislice CT is capable of acquiring images with $256 \times 256 \times 64$ dimensions and resolutions of $0.625 \mathrm{~mm}, 8$ times per second. Similarly, advances in transducer technology have led to improvements in the field of 3D ultrasound imaging, which can now acquire images with $128 \times 128 \times 128$ dimensions and resolution of $1 \mathrm{~mm}, 20$ times per second. These intraprocedural images, acquired during the procedure for navigation, represent the most current anatomical 
information but often suffer from poor signal-to-noise ratio. To achieve desired accuracy for IGIs, these intraprocedural 3D images, therefore, must be preprocessed and enhanced before they can be used for advanced image processing operations such as segmentation, registration, and visualization. Toward this end, anisotropic diffusion filtering and median filtering have been shown to be effective in enhancing and improving the visual quality of these images. It is important to note that the interactive nature of IGIs necessitates equivalent image processing speed so that these procedures can be performed in a streamlined manner without any additional processing latency.

The aforementioned filtering techniques are based on neighborhood (window) operations. For volumetric (3D) images, these neighborhoods are considerably larger $\left(\mathrm{N}^{3}\right)$, thus increasing the complexity of filtering operations. This complexity, coupled with the sheer size of intraprocedural volumetric images, results in execution times of several seconds for software implementations running on general-purpose workstations (Table 3.1). This processing speed is only a fraction of the acquisition speed of the intraprocedural images and is clearly unacceptable to meet the real-time

Table 3.1: $\quad$ Software execution time of 3D anisotropic diffusion filtering and 3D median filtering of 8-bit images for common kernel sizes $(N)$.

\begin{tabular}{|c|c|c|c|c|}
\hline Filter kernel & $\begin{array}{l}\text { Kernel } \\
\text { size }(N)\end{array}$ & $\begin{array}{l}\text { Image size } \\
\text { (voxels) }\end{array}$ & $\begin{array}{l}\text { Execution } \\
\text { time } \\
\text { (seconds) }\end{array}$ & $\begin{array}{c}\text { Voxel } \\
\text { processing } \\
\text { rate } \\
(\mathbf{M H z}) \\
\end{array}$ \\
\hline \multirow{2}{*}{$\begin{array}{l}\text { 3D anisotropic } \\
\text { diffusion filter }\end{array}$} & \multirow{2}{*}{7} & $128 \times 128 \times 128$ & 2.28 & 0.92 \\
\hline & & $256 \times 256 \times 64$ & 4.58 & 0.92 \\
\hline \multirow{4}{*}{$\begin{array}{l}\text { 3D median } \\
\text { filter }\end{array}$} & \multirow{2}{*}{3} & $128 \times 128 \times 128$ & 0.85 & 2.46 \\
\hline & & $256 \times 256 \times 64$ & 1.59 & 2.63 \\
\hline & \multirow{2}{*}{5} & $128 \times 128 \times 128$ & 3.01 & 0.7 \\
\hline & & $256 \times 256 \times 64$ & 5.67 & 0.74 \\
\hline
\end{tabular}


requirements of IGIs. Previously reported techniques for accelerated implementation of these filtering operations primarily focused on one-dimensional (1D) or twodimensional (2D) filters [7-11], with only a few implementations attempting to accelerate these operations in 3D.

This dissertation presents an FPGA-based architecture for real-time processing of intraprocedural 3D images. Earlier attempts to accelerate 3D anisotropic diffusion filtering were targeted toward multiprocessor clusters [100, 101]. Despite the near-linear speedup offered by these techniques, the need to employ up to 256 processors to achieve real-time performance makes them less suitable for clinical deployment. In this dissertation, we introduce a novel FPGA-based implementation of 3D anisotropic diffusion filtering. The developed solution is compact, easily deployable, and capable of processing the intraprocedural images faster than acquisition speeds. Some researchers have recently reported high-speed implementations of 3D median filtering using graphics processing units [136] and FPGAs [137]. This work presents an FPGA-based 3D median filtering module that is faster than currently existing solutions and supports higher 3D kernel sizes $(3,5,7)$. The designed architecture can achieve a processing rate close to 200 Megavoxels per second for both the 3D anisotropic diffusion and 3D median filtering, which is equivalent to about 50 processing iterations or operations per second for images of size $256 \times 256 \times 64$. Consequently, this design is capable of meeting the real-time data processing need of most IGIs. 


\subsection{Filtering Algorithms}

This section briefly describes the 3D image preprocessing algorithms that are being accelerated in the current work. The architecture for their real-time implementation is presented in the subsequent section.

\subsubsection{Anisotropic Diffusion Filtering}

As described earlier, anisotropic diffusion filtering is an iterative process which progressively smoothes an image $(I(\bar{v}, t))$ while maintaining the significant edges. This process can be summarized by the following equation:

$$
\frac{\partial I}{\partial t}=\operatorname{div}(c(\bar{v}, t) \cdot \nabla I(\bar{v}, t))
$$

where $c$ is the diffusion coefficient. Since the intraprocedural images typically have poor SNR, the current implementation employs Gaussian-filtered version of the image to estimate the gradient values, as proposed by Whitaker and Pizer [82]. The corresponding discrete expression, which is implemented in our design, for this filtering operation (shown for a 2D case for simplicity) is:

$$
I(x, y, t+\Delta t)=I(x, y, t)+\Delta t \cdot\left(\begin{array}{c}
c\left(\left|I_{G}(x+1, y, t)-I_{G}(x, y, t)\right|\right) \cdot[I(x+1, y, t)-I(x, y, t)] \\
+c\left(\left|I_{G}(x-1, y, t)-I_{G}(x, y, t)\right|\right) \cdot[I(x-1, y, t)-I(x, y, t)] \\
+c\left(\left|I_{G}(x, y+1, t)-I_{G}(x, y, t)\right|\right) \cdot[I(x, y+1, t)-I(x, y, t)] \\
+c\left(\left|I_{G}(x, y-1, t)-I_{G}(x, y, t)\right|\right) \cdot[I(x, y-1, t)-I(x, y, t)]
\end{array}\right),
$$

where $I_{G}$ is the Gaussian-filtered version of the image, $c$ is the discrete realization of the chosen diffusion function, and the time step $\Delta t$ controls the rate and stability of the diffusion process. Gerig et al. [138] calculated maximum values for $\Delta t$ for different neighborhood structures. For a 3D realization, diffusion is calculated in a 3D 
space with 6-connected neighborhood, and that configuration corresponds to a maximum $\Delta t$ value of $1 / 7$, which is implemented by the presented design.

\subsubsection{Median Filtering}

The 3D median filter design presented in this dissertation is based on a combination of bit-serial searching and majority voting approaches. This section describes this median finding scheme by means of an example. The algorithm is executed in $b$ (for $b$-bit images) steps, where each step finds 1 bit of the resulting median value starting from the most significant to the least significant bit. Specifically, at the $j^{\text {th }}$ step, the majority bit (' 0 ' or ' 1 ') amongst the $j^{\text {th }}$ significant bits of all the input elements in the neighborhood is calculated and represents the $j^{\text {th }}$ bit of the median of the neighborhood $(0 \leq j \leq b-1)$.

At a given step, when a bit of a input element differs from the majority bit calculated at that step, the bit value for that element is fixed in subsequent steps and is considered to be masked with its current bit value. Bits already masked in a previous step are not altered in subsequent steps (i.e., if the $j^{\text {th }}$ bit of input value $n$, represented using $b$ bits as: $n_{b-1} n_{b-2} \cdots n_{0}$, is masked, then the algorithm considers $\left.n_{i}=n_{j}, \forall i<j\right)$. The process of finding the median using this approach is illustrated in Figure 3.1. In this example, for simplicity, we consider a small input neighborhood consisting of only five elements with four bits per voxel ( $b=4)$; therefore, only four processing steps are required. Processing starts at the MSB position of the data elements. The bits of the data elements being considered for calculating the majority bit at any step are indicated in gray in the figure. The masking operation that takes 


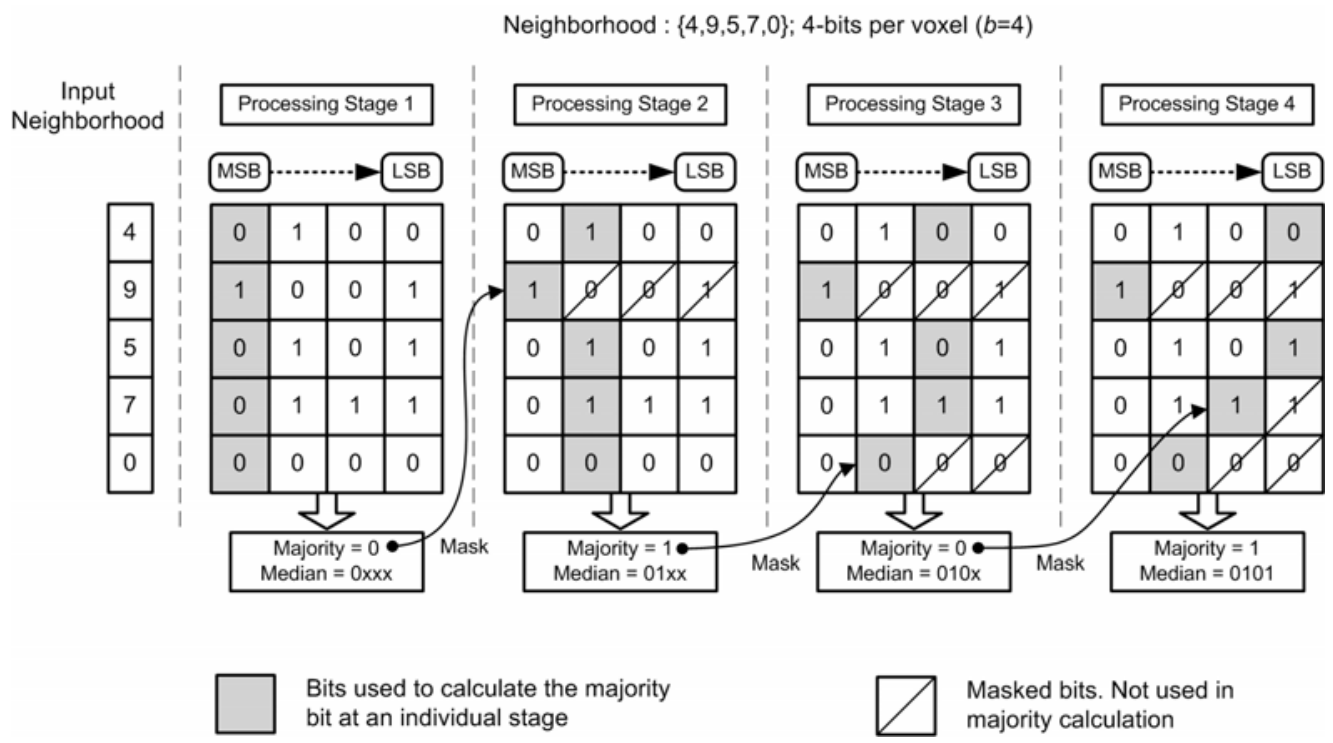

Figure 3.1: A median filtering example using majority voting technique.

place at the end of every step is indicated by arrows. The masked bits are shown to be crossed out. One bit of the median is determined at every processing stage starting from the MSB position, and the results from all the stages are combined to produce the final median value.

\subsection{Architecture}

We present an FPGA-based architecture that is capable of performing 3D anisotropic diffusion and 3D median filtering of intraprocedural images faster than their acquisition speed. A top-level block diagram of this architecture is shown in Figure 3.2. Input and output images are stored in two independent external memory banks, and the memory controller, input and output image buffers, and the filtering modules are implemented using an FPGA. The presented architecture supports two filtering modules, one for 3D anisotropic diffusion filtering and the other for 3D median filtering. The filtering module can be selected and reconfigured statically, whereas the memory controller and the image buffers are designed to be common to 


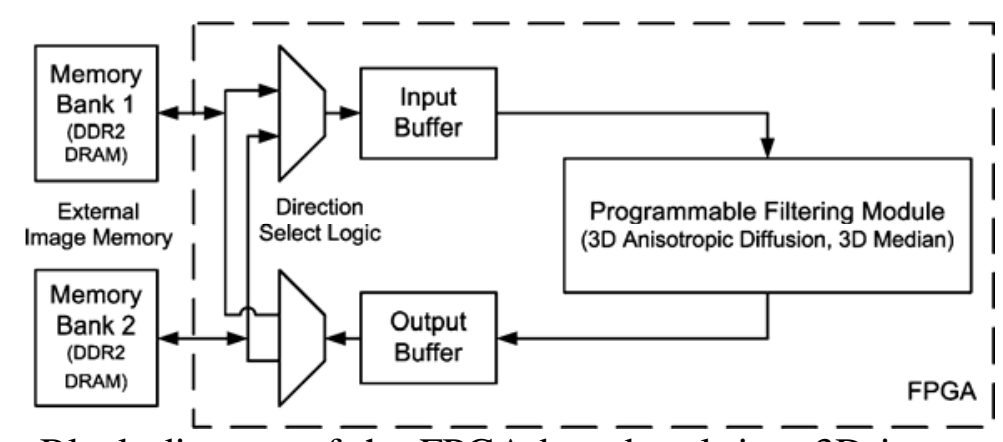

Figure 3.2: $\quad$ Block diagram of the FPG $\overline{A-b} \overline{\text { ase }} \overline{-} \overline{\text { real-time }} \overline{3 \mathrm{D}}$ image preprocessing system.

all supported filtering modules. The role of input and output memory banks can be switched at runtime, thus enabling execution of consecutive filtering operations (or iterations) without additional data transfers between the memory banks.

In order to achieve real-time performance, it is imperative to aim at a throughput of one processed (output) image voxel per clock cycle. Because both anisotropic diffusion and median filtering involve neighborhood operations, meeting this throughput requirement is challenging, given that an entire neighborhood $\left(N^{3}\right.$ voxels, where $N$ is the filter kernel size) must be accessed in order to compute one output voxel. Moreover, adjoining neighborhoods must be continuously fetched from the input memory bank as next output voxels are computed sequentially. These neighborhoods are read by the memory controller from the input image memory bank and are stored into the input buffer in an $N \times N \times N$ arrangement.

The filtering module receives the neighborhood to be processed in a pipelined fashion: $N \times N$ new voxels every clock cycle. Once the filtering module pipeline is full (after $N$ clock cycles), the filtering module computes one output voxel per clock cycle. The sequential input neighborhoods are continuously processed, and the resulting output voxels are stored into the output buffer. The memory controller then 
transfers these resultant voxels to the output image memory in a burst of one image row at a time. The memory controller uses a brick-caching scheme, specifically devised to meet the high input data rate required by this task. This brick-caching scheme takes advantage of the fact that adjacent neighborhoods share $N \times N \times(N-1)$ voxels and only $N \times N$ new voxels need to be supplied every clock cycle for continuous neighborhood processing. The implementation of this scheme is described in the following sections. For the remainder of this chapter, we use the following notations: image dimensions are represented as $N_{x} \times N_{y} \times N_{z}$. The parameter $b$ indicates the number of bits used to represent the voxel intensity in the image, and $N$ is the filter kernel size with corresponding neighborhood size of $N^{3}$. .Input and output images are arranged in the memory banks along the $z-y-x$ order, with rows of the memory aligned with the $z$ direction of the image. The output voxels are also calculated in $z-y-x$ order.

\subsubsection{Memory Controller and Brick-caching Scheme}

Memory organization and neighborhood access techniques are often the limiting factors in 3D image processing systems [139-142]. However, most practical filtering techniques employ standard neighborhood operations that require block-
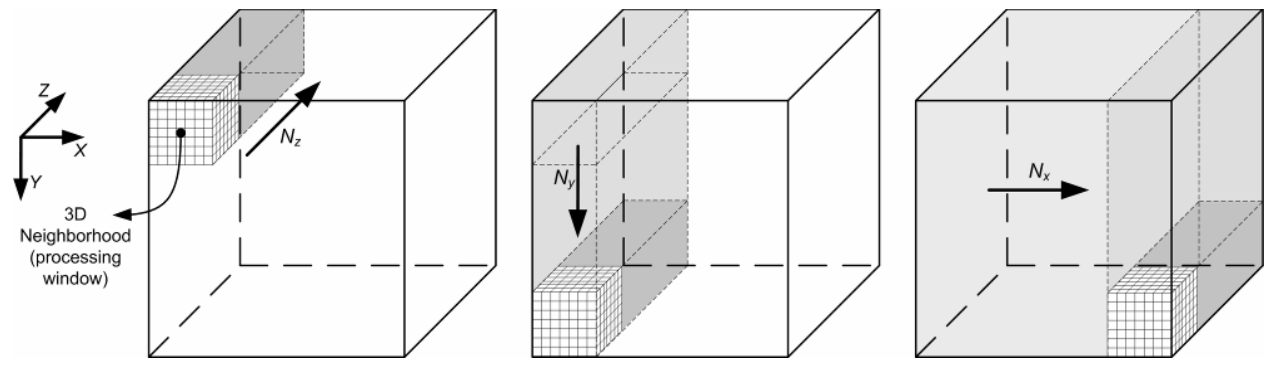

Figure 3.3: Typical voxel access pattern for neighborhood operations-based image processing. 
sequential voxel access, as shown in Figure 3.3. The presented FPGA-based architecture uses a raster scan order distribution of voxels in the image memory, along with a brick-caching scheme to take advantage of this block-sequential access pattern. For every output voxel calculation, an entire neighborhood of $N \times N \times N$ voxels must be accessed. This neighborhood cannot be retrieved in a single burst access of a sequentially organized image memory. Moreover, in a pipelined implementation, data must be continuously fetched for successive neighborhood operations. To sustain the high data rate required to achieve real-time processing speeds, this architecture employs a brick-caching scheme that loads the image into the input buffer that stores an $N \times(N+1)$ array of image rows (i.e., it stores up to $N \times(N+1) \times N_{z}$ voxels). This input buffer is implemented using high-speed and dualported memory blocks internal to the FPGA. The input buffer can be accessed in a single clock cycle, which enables fast updates and reads. Figure 3.4 shows the block diagram of the input image memory and the input buffer, which consists of an $N \times(N+1)$ array of internal memory blocks, each holding $N_{z}$ voxel intensity values. We use the following terminology: a brick is an $N \times N \times N_{z}$ block of image intensity values stored in the internal buffer. A brick plane is an $N \times 1 \times N_{z}$ section of a brick. A brick slice is an $N \times N \times 1$ section of a brick. A brick row (or simply row) is a $1 \times 1 \times N_{z}$ section of a brick. Each row corresponds to and contains one input image row containing $N_{z}$ voxels. The pictorial representation of this terminology is shown in Figure 3.5. Bricks are loaded into the buffer one brick row at a time for an available brick plane and are then fed to the filtering pipeline one brick slice at a time. 


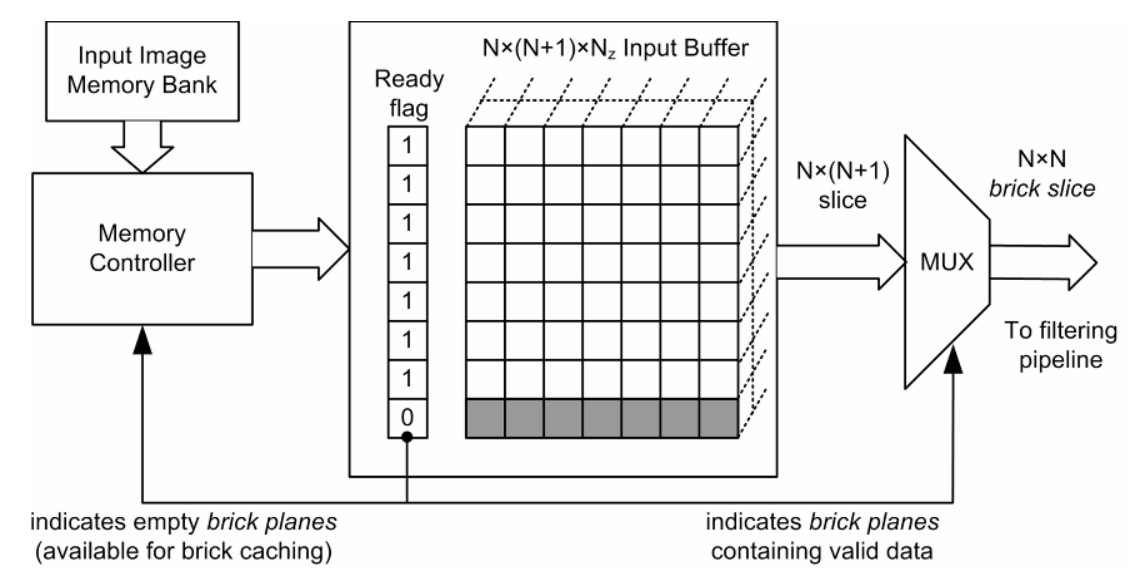

Figure 3.4: Block diagram showing the input image memory and the input buffer configuration.

The input buffer, therefore, can store a whole brick plus an extra brick plane. The brick-caching operation is described below.

The memory controller fills up the input buffer row by row. Each row contains $N_{z}$ voxels, and thus transfer of each row takes $t_{\text {Row }}=t_{\text {lat }}+N_{z} / W$ clock cycles, where $t_{\text {lat }}$ is the number of clock cycles necessary to start a burst memory transfer and $W$ is the effective data bus width in terms of number of voxels (e.g., double-data-rate [DDR] dynamic random access memory [DRAM] will offer twice the effective bus width of single-data-rate DRAM with a similar configuration). After the first row is cached in, the controller starts caching the row next to it in the $x$ direction. A complete brick plane ( $N$ image rows, $N \times 1 \times N_{z}$ voxels) can be loaded in $t_{\text {Plane }}=N \cdot t_{\text {Row }}$ clock cycles. Associated with every brick plane is a ready flag. This flag serves a dual purpose; when ' 1 ', it indicates the availability of data for that particular brick plane, and, when ' 0 ', it indicates that the brick plane is empty and available for caching image voxels. After one brick plane is loaded into the input buffer, the memory controller sets the corresponding ready flag and starts loading the 


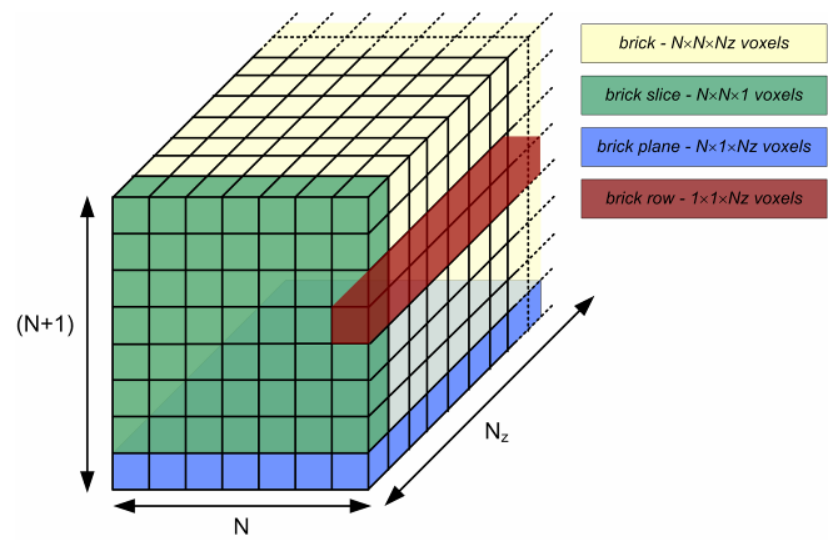

Figure 3.5: Pictorial representation of the notation used in the brick-caching scheme.

next brick plane (along the $y$ direction). Once a complete $N \times N \times N_{z}$ brick is available in the input buffer, it is fed into the filtering module pipeline one brick slice $(N \times N$ voxels) at a time.

The filtering module pipeline operates on one $N \times N \times N$ neighborhood at a time and is fed with a new brick slice every clock cycle. Loading an entire $N \times N \times N_{z}$ brick into the filtering module pipeline thus takes $N_{z}$ clock cycles. While this operation is in progress, the memory controller loads the next brick plane (along the $y$ direction) into the buffer plane that is not being used for processing (indicated by the ready flag), which requires $t_{\text {Plane }}$ clock cycles. After processing of all the neighborhoods in the $N \times N \times N_{z}$ brick is complete, the processing window shifts along the $y$ dimension of the image, and the processing of the new neighborhoods begins. Simultaneously, the ready flag corresponding to the brick plane that is no longer used is set to ' 0 '. This available brick plane in the input buffer is then used for caching the next image rows (along $y$ direction). In this fashion all brick planes in the input buffer are cyclically used for brick caching during processing. These steps 
continue until the processing window reaches the end of the column (i.e., until $y=$ $N_{y}$ ). At this point, the processing window moves along the $x$ direction. To accomplish this, data in the internal buffers are invalidated, and the complete $N \times N \times N_{z}$ brick in the next column is cached, which requires a pipeline stall. After the initial brick in the new $x$ coordinates is loaded, the processing continues as described earlier. The processing of the entire 3D image is completed accordingly. For continuous pipelined operation with minimum stalls, the memory controller must provide the next brick plane before the processing of the previous brick is completed. Therefore, the relationship expressed in the following equation must be met:

$$
N \cdot\left(t_{\text {lat }}+N_{z} / W\right) \cdot T_{\text {mem }} \leq N_{z} \cdot T_{\text {proc }} \text {, }
$$

where $T_{m e m}$ is the clock period of the external memory clock and $T_{\text {proc }}$ is the clock period of the internal filtering pipeline. The left-hand side of Eq. (3.3) refers to the total time required to load a new brick plane. The right-hand side refers to the total time required to process a whole brick. Assuming that efficient burst accesses (supported by most modern dynamic memories) are being used (which implies: $t_{\text {lat }} \ll N_{z} / W$ ), the following relationship must be maintained to minimize pipeline stalls:

$$
N \cdot T_{\text {mem }} \leq W \cdot T_{\text {proc }} \cdot
$$

\subsubsection{D Anisotropic Diffusion Filtering}

This architecture supports 3D anisotropic diffusion filtering by pipelined implementation of the 3D extension of the formulation shown in Eq. (3.2). As indicated by that formulation, we use a Gaussian-filtered version of the image for 
improved diffusion coefficient estimation. Our design implements this Gaussian filtering at runtime using an embedded 3D Gaussian filtering module. Figure 3.6 shows a top level block diagram of the 3D anisotropic diffusion filtering module. This filtering pipeline operates on $N \times N \times N$ voxel neighborhoods. On each clock cycle, the input data buffer feeds an $N \times N$ voxel neighborhood (brick) slice into the pipeline. The center voxel intensity value is passed to the delay element for accumulation at the end of the pipeline per Eq. (3.2). The $3 \times 3$ voxel neighborhood located at the center of the incoming $N \times N$ neighborhood is passed to the image gradient calculator, which calculates the image gradients with respect to each of the 6-connected neighbors of the center voxel. The embedded Gaussian filtering module calculates, in parallel, the Gaussian-filtered values for each of the six-connected neighbors and passes them to the diffusion coefficient calculator, which calculates the diffusion coefficients $c_{0.5}$ corresponding to each of the input gradients. Taking advantage of the parallelism native to FPGAs, these operations are executed in

parallel, and, as a result, this filtering module can calculate the output at the rate of one voxel per clock cycle. The resulting voxel intensity values are fed into the output buffer, and the memory controller then stores them in the output memory bank.

\subsubsection{Embedded Gaussian Filtering Module}

Equation (3.5) shows the formula to calculate the coefficients of a 3D Gaussian filter kernel, where $\sigma$ is the standard deviation of the Gaussian function and $d$ is the Euclidean distance between the desired coefficient location and the kernel center. For a chosen $\sigma$, the coefficient values depend exclusively on the Euclidean 


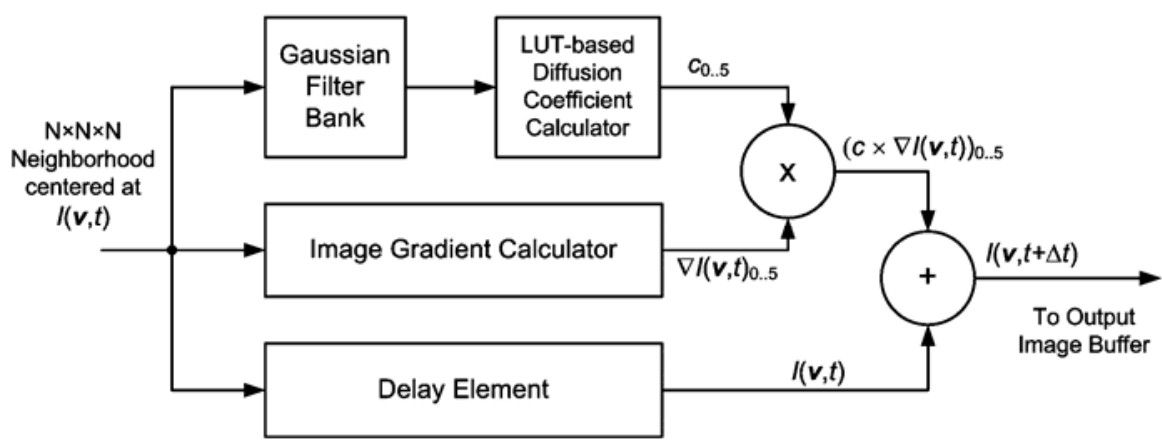

Figure 3.6: Top-level block diagram of 3D anisotropic diffusion filtering. This diagram indicates paths that are executed in parallel.

distances from the kernel center; thus, the Gaussian filter kernel exhibits symmetries with respect to its center (i.e. it is radially symmetric).

$$
G_{d}(\sigma)=\exp \left(-\frac{d^{2}}{2 \sigma^{2}}\right)
$$

Our architecture takes advantage of these symmetries to reduce the number of multipliers needed for implementing the 3D Gaussian kernel to $k \times(k+1) \times(k+2) / 6$ from $(N-2)^{3}$; where $N$ is the size of anisotropic diffusion filtering kernel, the corresponding size of the embedded Gaussian filtering kernel is $(N-2)$, and $k=(N-1) / 2$. For example, a $5 \times 5 \times 5$ embedded Gaussian kernel that arises in anisotropic diffusion filtering with $N=7$ can be implemented using only 10 multipliers (as opposed to 125) as we reported previously [143]. For this kernel, each individual slice ( $5 \times 5$ plane of the kernel) has six isodistance regions and the whole 3D kernel has 10. During filtering operation, all voxels that are equidistant from the kernel center are multiplied against the same Gaussian coefficient. The intensities corresponding to these voxels in the same isodistance region can, therefore, be preadded before being multiplied against the Gaussian coefficients. Because a $5 \times 5 \times 5$ 
Gaussian kernel contains 10 isodistance regions, the minimum number of multipliers necessary to implement this filter kernel is, therefore, 10.

A block diagram of the Gaussian filter bank is shown in Figure 3.7. On each clock cycle, the input buffer feeds an $N \times N$ voxel neighborhood into the bank. This neighborhood is decomposed into five $(N-2) \times(N-2)$ neighborhoods by the input demux, and these neighborhoods are then passed to five embedded 3D Gaussian filters. Figure 3.8 shows a block diagram of an embedded 3D Gaussian filter. The pre-adder accumulates values corresponding to the isodistance groups in the incoming $(N-2) \times(N-2)$ neighborhood, thus compressing the neighborhood based on the intraneighborhood plane isodistance criterion (e.g., each single $5 \times 5$ slice of a $5^{3}$ Gaussian neighborhood has 6 isodistance regions). These pre-added values are then passed to $(N-2)$ pipeline buffers, which make values corresponding to the entire $(N-2)^{3}$ neighborhood available in parallel. The sorter-accumulator aggregates these values corresponding to the isodistance groups between the slices,

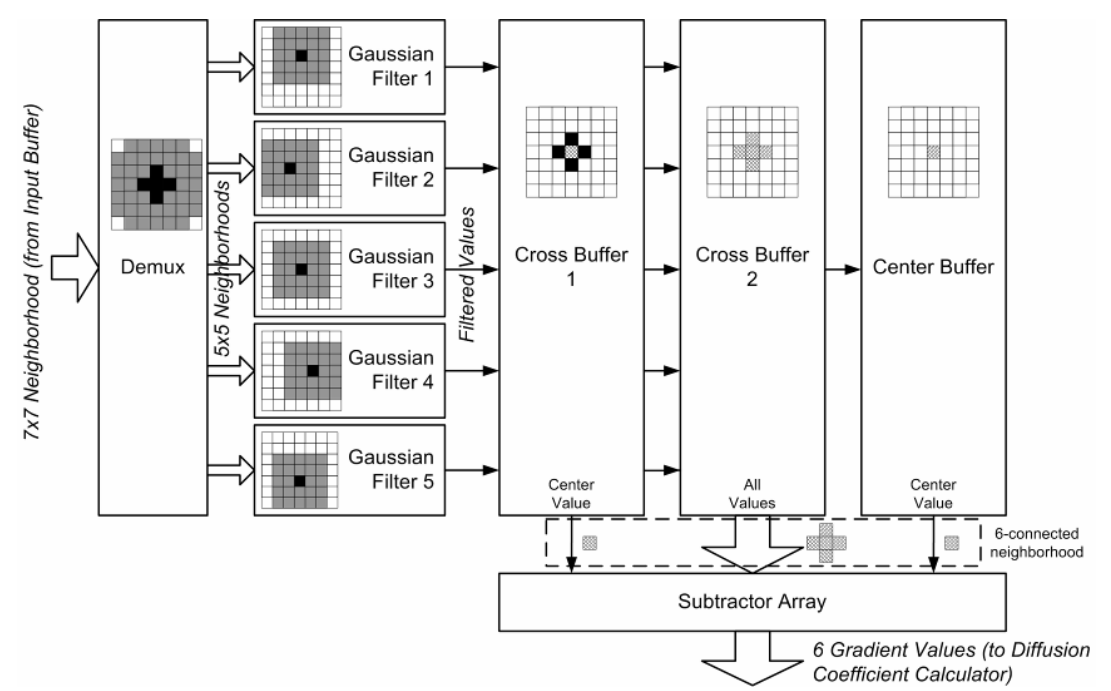

Figure 3.7: Block diagram of the embedded Gaussian filter bank (for $\mathrm{N}=7$, corresponding Gaussian kernel size is 5). 


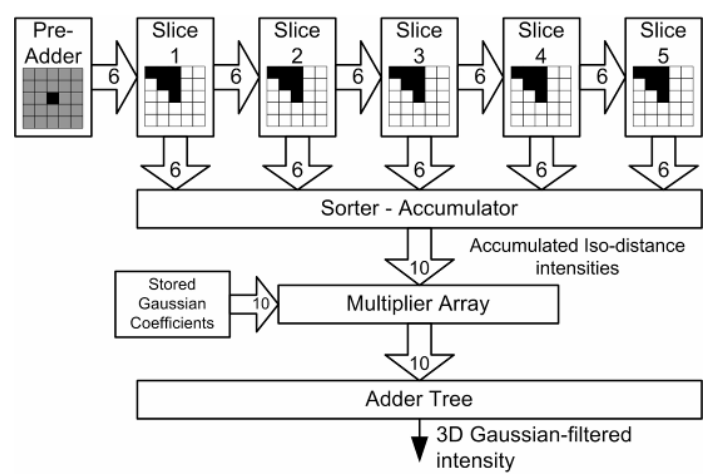

Figure 3.8: Pipelined implementation of an individual Gaussian filter element (Gaussian kernel size $=5$ ).

compressing them further using the isodistance criterion for the entire neighborhood (e.g., 10 values that correspond to the 10 unique coefficients in a $5 \times 5 \times 5$ Gaussian neighborhood). These values are passed to the multiplier array, where they are multiplied against their corresponding Gaussian coefficients. The adder tree then adds the resulting values and outputs the result for the current 3D neighborhood. The results for the subsequent neighborhoods are produced continuously as a result of pipelined implementation of the operation. The Gaussian coefficients are precomputed for a given value of $\sigma$ and are stored in internal registers using fixedpoint representation. The effect of this finite precision representation is analyzed in the flowing section.

The results from the five individual Gaussian filters correspond to a crossshaped region of a neighborhood slice. In order to operate on the 3D six-connected neighborhood, these results are passed to pipelined registers composed of two cross buffers and the center buffer. The cross buffers store all five values in a neighborhood slice, whereas the center buffer stores only the center value. As a result, the entire sixconnected neighborhood is available between these buffers. The buffers then send the Gaussian-filtered, six-connected 3D voxel neighborhood to the subtractor array, 
which calculates the six corresponding gradient values and passes them to the diffusion coefficient calculator.

\subsubsection{Diffusion Coefficient Calculation}

As noted previously, gradients calculated after Gaussian filtering are used to estimate the diffusion coefficients. For a $b$-bit image, the absolute value of the gradient is limited to the range 0 and $2^{b}-1$. Taking advantage of this fact the desired diffusion function is discretized in $2^{b}$ steps and implemented using a lookup table (LUT). The use of a LUT allows an efficient implementation of any diffusion function. It must be noted that, because the dynamic range of all diffusion functions is limited to $[0,1]$, there is no significant loss in precision by a using a fixed-point representation. The effect of this finite precision representation is further analyzed in the following section.

\subsubsection{Image Gradient and Result Calculation}

Image gradient calculation is performed by an array of six parallel subtractors. These subtractors calculate the difference between the intensity of the voxel located in the center of the kernel against its six-connected neighbors. These values are then multiplied against their corresponding diffusion coefficients (supplied by the diffusion coefficient calculator) using an array of six parallel multipliers. The resulting filtered intensity is then obtained by adding the six results from the multipliers to the original center voxel intensity. After rounding and truncation, this result is then sent to the output buffer and is then further saved into output memory bank. 


\subsubsection{Median Filtering}

The 3D median filtering design presented in this work is an extension of majority finding-based implementation proposed by Benkrid et al.[7]. That design was reported for a $2 \mathrm{D}$ realization and computed only one bit of the median value per clock cycle. All bits of the median value were obtained using a feedback loop and hence for $b$-bit images, this approach required $b$ clock cycles to compute the resulting median value. The implementation presented in this dissertation extends that design to 3D and unrolls the feedback loop by using multiple processing stages. Moreover, our implementation exploits the regularity of this median finding algorithm with a systolic array architecture that allows a pipelined implementation and, therefore, can achieve a throughput of one median value per clock cycle. Thus, our implementation can achieve a voxel processing speed $b$ times higher than the previously reported architecture [7]. Our linear systolic array employs $b$ identical processing stages for filtering a $b$-bit image. Figure 3.1 illustrates execution of this algorithm for a small example and can be used to gain further insights into its hardware implementation. Each processing stage of our systolic array implementation corresponds to one step of the algorithm execution. Starting from the MSB, each stage generates one bit of the resulting median value of the neighborhood being processed. We first describe the operation of an individual processing element and then explain the functioning of the entire linear systolic pipeline, which contains $b$ processing elements. 


\subsubsection{Processing Element}

The processing element is the atomic unit of the proposed linear systolic array design. A functional block diagram of the processing element at the $j^{\text {th }}$ stage is shown in Figure 3.9. The data inputs to this processing element are Data_Bits $j_{j}$ and Next_Data_Bits , the $\mathrm{N}^{3}$ bits used considered for majority calculation and the $(j+1)^{\text {th }}$ significant bits (from MSB) of the $N^{3}$ neighborhood elements, respectively. It must be noted that, although Next_Data_Bits j are corresponding image intensity bits, Data_Bits $\mathrm{j}$ are provided by the $(\mathrm{j}-1)^{\mathrm{th}}$ processing stage and may have been masked in the earlier stages. The accompanying input Mask_Bits $\mathrm{j}$ is a binary flag that indicates the bits in Data_Bits that have been masked in the prior stages. A processing element performs two important tasks. First, it computes the majority bit within the $\mathrm{N}^{3}$ input data bits (Data_Bits ); second, it performs the masking operation based on the majority bit calculated and outputs masked data bits (Data_Bits ${ }_{j+1}$ ) and the corresponding binary flag (Mask_Bits ${ }_{j+1}$ ) to be used in the next processing stage. The units that perform these two operations are described below.

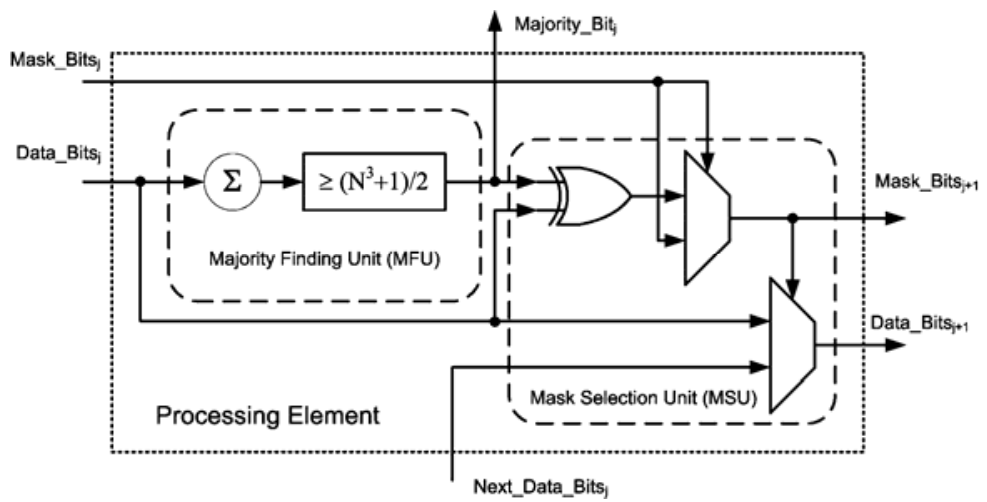

Figure 3.9: A single stage (processing element) of the linear systolic median filtering kernel. 


\section{Majority Finding Unit (MFU)}

The MFU consists of a bit-counting circuit that counts the number of $1 \mathrm{~s}$ in the input bits that are considered for majority calculation (Data_Bits $\mathrm{j}_{\mathrm{j}}$ ). This counting is performed using a bit adder tree customized for a chosen neighborhood size. This count is then compared against a threshold, which is programmed to be half of the number of elements contained in the neighborhood. The binary result of this

comparison is the $j^{\text {th }}$ significant (from MSB) bit of the output median value. The highly compact, pipelined, and customized implementation of the MFU minimizes the combinational delay within the processing element.

Mask Selection Unit (MSU)

After the median bit has been calculated, the MSU performs the masking operation. It computes the mask bit for each bit of Data_Bits, based on whether it matches with the majority bit or not. In addition, it considers and preserves the bits that were masked in the prior stages (Mask_Bits $\mathrm{j}$ ). Thus, the mask calculated at the $\mathrm{j}^{\text {th }}$ stage (Mask_Bits $j_{j+1}$ ) is a combination of the mask bits from the prior stages and the mask calculated at the current stage. This masking operation is implemented using an exclusive OR (XOR) operation and two multiplexing operations for each data bit. The calculated mask (Mask_Bits $j_{j+1}$ ) is then used to selectively generate Data_Bits $j_{j+1}$ from the input Next_Data_Bitsj, while ensuring that values corresponding to the masked bits are preserved. Data_Bits $\mathrm{s}_{\mathrm{j}+1}$ is then used in the next processing stage to calculate the $(j+1)^{\text {th }}$ significant bit of the median value. 


\subsubsection{Linear Systolic Design for Median Finding}

The proposed linear systolic design is realized by cascading $b$ processing elements for filtering $b$-bit images. On every clock cycle, a complete neighborhood containing $\mathrm{N}^{3}$ voxels, $b$-bits each, is fed to this linear systolic array. However, the processing stage $(\mathrm{j}+1)$ can not perform its operation until stage $\mathrm{j}$ finishes its processing and provides Data_Bits $j_{j+1}$ and Mask_Bits $\mathrm{j}_{\mathrm{j}+1}$. Similarly, stage j produces its output ( $\mathrm{j}^{\text {th }}$ significant bit of the median) one clock cycle earlier than the corresponding output by the stage $(\mathrm{j}+1)$. In order to compensate for these delay and processing latencies and to provide synchronized operation, additional line delay units and data shift registers must be inserted at the input and output of the systolic array. Figure 3.10 shows a diagram of this configuration with $b$ processing elements and required delay buffers. These delays are introduced for synchronization only, and it must be noted that as long as the input sequential neighborhoods are continuously supplied (new $\mathrm{N}^{3}$ voxels every clock cycle), the systolic array design is capable of computing one median result per clock cycle. This result is then sent to the output buffer and subsequently saved into the output memory bank by the memory controller.

For correct operation, the Mask_Bits ${ }_{1}$ input of the first processing stage (stage 1, MSB) is grounded (set to "0"), indicating that no bits from the input data (Data_Bits ${ }_{1}$ ) are masked. Also, in the final processing stage (stage b, LSB), Mask_bits $\mathrm{b}_{\mathrm{b}+1}$ and Data_Bits $\mathrm{b}_{\mathrm{b}+1}$ do not need to be calculated, because the next stage does not exist. Consequently, the MSU is not needed in the final stage, which contains only an MFU to compute the last median bit. In general, for large 3D neighborhoods, the speed of the MFU is the limiting factor of the systolic array 


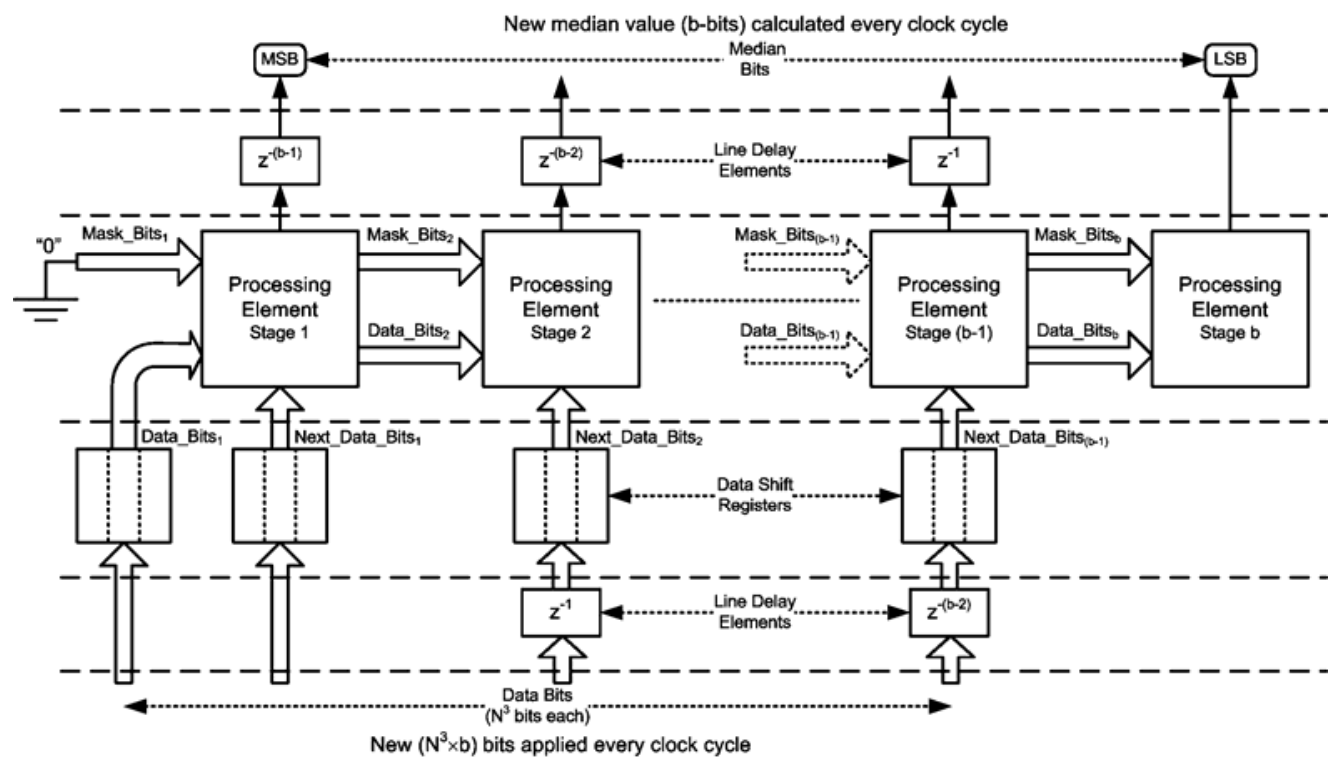

Figure 3.10: Linear systolic array architecture for median filter kernel using majority voting technique.

performance. In applications requiring high voxel throughput and large filtering kernels, the operation of the MFU can be pipelined. However, in those cases the depth of the delay elements used to synchronize the inputs and outputs of the different processing elements must be adjusted.

\subsection{Implementation and Results}

The architecture described above was implemented using an Altera Stratix II EP2S180F1508C4 FPGA (Altera Corp., San Jose, CA) with two external memory banks to serve as input and output image memory. The memory banks used were 1GB DDR2 small-outline dual-inline memory DRAM modules with 64-bit data bus (i.e., $W=16$, for $b=8$ ) running at a $200-\mathrm{MHz}$ clock speed. The architecture was designed using VHSIC hardware description language (VHDL) and synthesized using Altera Quartus II 6.1. The memory controller was also implemented using VHDL and was built around the DDR2 DRAM controller megacore supplied with Altera Quartus 
II. Both filtering modules were custom designed using VHDL, as per the design description in provided earlier. Functional verification and postsynthesis timing simulation for the entire system were performed using Modelsim SE 6.2 (Mentor Graphics, San Jose, CA). For this purpose, DDR2 DRAM simulation models provided by Micron (www.micron.com) were used. The presented design was then realized to support 8-bit images $(b=8)$ and, consequently, all results in this section are presented for 8-bit images. The execution speed of the presented architecture was obtained from postsynthesis timing simulation of the design.

\subsubsection{Effects of Finite Precision Representation}

Real-time filtering performance offered by the presented design is critical for the time-sensitive nature of IGIs, but of equal importance is the accuracy of the filtering process. Most software implementations represent the arithmetic operations involved in the filtering algorithms using a double precision floating-point format. This format offers high dynamic range and precision, which may or may not be required depending on the filtering technique to be implemented. Median filtering, for example, is performed exclusively using integer data (because digital images are represented using $b$-bit integer data), and, hence, there is no loss in precision by using

Table 3.2: $\quad$ Average error in intensity per voxel for a Gaussian filtered image resulting from fixed-point representation of Gaussian coefficients.

\begin{tabular}{cccc}
\hline \hline $\begin{array}{c}\sigma \\
\sigma\end{array}$ of the \\
Gaussian kernel & \multicolumn{3}{c}{$\begin{array}{c}\text { Average error in intensity per voxel resulting from } \\
\text { fixed-point representation of Gaussian kernel coefficients }\end{array}$} \\
\cline { 2 - 4 } & 8-bits & 12-bits & 16-bits \\
\hline 0.3 & $0.20 \pm 0.47$ & $0.07 \pm 0.26$ & $0.004 \pm 0.06$ \\
0.5 & $0.63 \pm 0.68$ & $0.02 \pm 0.11$ & $0.004 \pm 0.07$ \\
0.7 & $0.42 \pm 0.81$ & $0.03 \pm 0.16$ & $0.003 \pm 0.06$ \\
1.0 & $0.21 \pm 0.47$ & $0.001 \pm 0.04$ & $0.001 \pm 0.03$ \\
\hline
\end{tabular}


a fixed-point implementation with sufficient dynamic range (i.e., using $b$ bits for $b$-bit images). Our implementation of the 3D median filtering uses $b$-bit integer representation for $b$-bit images, and therefore provides identical results to those provided by a software implementation.

Anisotropic diffusion filtering, however, involves operations with the data in real format. Our implementation, for the sake of efficiency in area and execution speed, used fixed-point representation to implement these arithmetic operations. A general framework for analyzing optimized tradeoff relationships between hardware resources and implementation accuracy for finite-precision designs is presented later (see Chapter 5). For this implementation, however, given the simplicity of the arithmetic operations and relatively minor impact of the fixed-point datapath on the total hardware resource requirement, we employed simulation-based wordlength search techniques. We analyzed the effect of the number of bits used for this fixedpoint representation on the filtering accuracy, by treating a software $(\mathrm{C}++)$ implementation employing double-precision floating-point representation as a reference. This analysis was performed with an 8-bit image with dimensions $256 \times 256 \times 64$. There are two sources at which error resulting from fixed-point precision can affect the accuracy of the filtering operation: embedded Gaussian

Table 3.3: Average error per sample of diffusion function resulting from fixedpoint representation of diffusion coefficients employed in the presented architecture.

\begin{tabular}{cccc}
\hline \multirow{2}{*}{$\boldsymbol{c}$} & \multicolumn{3}{c}{$\begin{array}{c}\text { Average error per sample of diffusion function resulting from } \\
\text { fixed-point representation of diffusion coefficients }\end{array}$} \\
\cline { 2 - 4 } & 8-bits & 12-bits & 16-bits \\
\hline 10 & $42 \times 10^{-5} \pm 97 \times 10^{-5}$ & $3 \times 10^{-5} \pm 7 \times 10^{-5}$ & $<10^{-5}$ \\
20 & $78 \times 10^{-5} \pm 114 \times 10^{-5}$ & $6 \times 10^{-5} \pm 8 \times 10^{-5}$ & $<10^{-5}$ \\
30 & $121 \times 10^{-5} \pm 125 \times 10^{-5}$ & $9 \times 10^{-5} \pm 8 \times 10^{-5}$ & $<10^{-5}$ \\
50 & $196 \times 10^{-5} \pm 116 \times 10^{-5}$ & $13 \times 10^{-5} \pm 7 \times 10^{-5}$ & $<10^{-5}$ \\
\hline \hline
\end{tabular}


filtering and diffusion function calculation. To gain additional insight, we evaluated accuracy for these individual sources and the accuracy of the anisotropic diffusion filtering as their combined effect.

Table 3.2 presents the average error in intensity per voxel after embedded Gaussian filtering (kernel size, $N=5$ ) where the Gaussian kernel coefficients are represented using the fixed-point format with the designated number of bits. Because the Gaussian kernel was normalized, all coefficients were within the range $[0,1]$, and, hence, we used one bit to represent the integer part and the rest for the fractional part. We performed this analysis for typical choices of $\sigma$ for a Gaussian kernel size of 5, which corresponds to the anisotropic diffusion filtering kernel size of 7 . The average error for various choices of $\sigma$ with 8-bit representation is less than one intensity value, and, as expected, the average error reduces with the increasing number of bits. It must be noted, however, that embedded Gaussian filtering is used only to estimate the diffusion coefficients, and, hence, small errors introduced in this operation may not have a significant impact on the final anisotropic diffusion filtered intensity value. Because this design supported 8-bit images, a 256-entry LUT was used to implement the diffusion function described in the background section. We implemented this function for reasonable choices of the parameter $K$, which controls the level of the gradient at which edges are diffused or preserved. The value of $K$ depends on the image modality and the amount of edge preservation desired. For ultrasound and lowdose CT images, however, its value is typically less than $20 \%$ of the intensity range. As the selected diffusion function takes values in the range $[0,1]$, we used one bit to represent the integer part and the rest for the fractional part. Table 3.3 presents the 
average error per sample of diffusion function resulting from fixed-point representation with the designated number of bits for various choices of $K$. Although the average error increases with the choice of $K$, its mean and standard deviations are consistently less than $0.1 \%$ of the data range, even with representation using 8-bits. Finally, Table 3.4 reports average error in intensity per voxel resulting from the combined effect of finite precision implementation of both the Gaussian coefficients and the diffusion function. For this analysis, we used the same number of bits for fixed-point representation of both entities, with one bit for the integer part and the rest for the fractional component. The kernel size $(N)$ of the anisotropic diffusion filter was chosen to be 7, with embedded Gaussian filtering with $\sigma=0.5$, and the diffusion function shown described earlier was implemented with $K=20$. To evaluate error accumulation over multiple iterations of anisotropic diffusion filtering, we performed this analysis up to 5 iterations, which is typical for filtering of intraprocedural images. The average error in intensity increases with the number of iterations, but its mean and standard deviations are consistently less than $0.04 \%$ of the intensity range, even with 8-bit representation.

Overall, our precision analysis indicates that even when using 8-bit fixed-

Table 3.4: $\quad$ Average error in intensity per voxel for anisotropic diffusion filtered resulting from fixed-point representation of Gaussian coefficients and the diffusion function

\begin{tabular}{cccc}
\hline \hline $\begin{array}{c}\text { Number of } \\
\text { filtering } \\
\text { iterations }\end{array}$ & \multicolumn{2}{c}{$\begin{array}{c}\text { Average error in intensity per voxel resulting from fixed-point } \\
\text { representation of the Gaussian kernel and diffusion coefficients }\end{array}$} \\
\cline { 2 - 4 } & 8-bits & 12-bits & 16-bits \\
\hline 1 & $0.008 \pm 0.092$ & $0.001 \pm 0.018$ & $<0.001$ \\
3 & $0.021 \pm 0.144$ & $0.001 \pm 0.031$ & $<0.001$ \\
5 & $0.030 \pm 0.171$ & $0.002 \pm 0.039$ & $<0.001$ \\
\hline \hline
\end{tabular}


point representation to perform Gaussian filtering and diffusion function calculation, the average error in intensity is only a very small percentage of the intensity range. Such small errors in intensity may not be significant for advanced operations such as registration, segmentation, and visualization and are unlikely to affect the accuracy and precision of IGIs. Our implementation, therefore, uses 8-bit fixed-point representation for these operations.

\subsubsection{Hardware Requirements}

Table 3.5 lists the significant hardware requirements for the important modules in the proposed architecture, parameterized on filter kernel size $(N)$ and the number of bits used to represent the voxel intensity $(b)$. The parameter $k$, introduced in the context of 3D anisotropic diffusion filtering, represents the number of unique isodistances in the Gaussian kernel and is related to the filter kernel size $N$ (usually odd) as:

$$
k=\frac{(N-1)}{2} .
$$

Table 3.5: Hardware requirements of the architecture for real-time 3D image preprocessing.

\begin{tabular}{ccc|ccc}
\hline \multirow{2}{*}{$\begin{array}{c}\text { Hardware } \\
\text { module }\end{array}$} & \multicolumn{2}{c|}{ Significant hardware resources } & \multicolumn{3}{c}{$\begin{array}{c}\text { Logic resources and performance } \\
\text { (as implemented) }\end{array}$} \\
\cline { 2 - 6 } & $\begin{array}{c}\text { Multipliers } \\
(b \times b \text { bit })\end{array}$ & $\begin{array}{c}\text { Internal memory } \\
\text { (bits) }\end{array}$ & $N$ & $\begin{array}{c}\text { Number of ALUTs } \\
(\% \text { utilization) }\end{array}$ & $\begin{array}{c}f_{\max } \\
(\mathbf{M H z})\end{array}$ \\
\hline $\begin{array}{c}\text { Input buffer } \\
\text { and controller }\end{array}$ & - & $\left(N \times(N+1) \times N_{z}\right) \times b$ & 7 & $1957(1.5 \%)$ & 233 \\
$\begin{array}{c}\text { Output buffer } \\
\text { and controller }\end{array}$ & - & $\left(2 \times N_{z}\right) \times b$ & 7 & $1743(1.5 \%)$ & 233 \\
$\begin{array}{c}\text { Anisotropic } \\
\text { diffusion filter }\end{array}$ & $5 \times \frac{k \times(k+1) \times(k+2)}{6}$ & $\left(3 \times 2^{b}\right) \times b$ & 7 & $3824(3 \%)$ & 236 \\
Median filter & - & - & 5 & $11308(8 \%)$ & 224 \\
\hline \hline
\end{tabular}


The linear systolic array implementation of the 3D median filter requires logic resources only, and the resource requirements for important components of this filter kernel are listed separately in Table 3.6. These two tables indicate how the hardware requirements of our architecture scale with the parameters $N$ and $b$. As dictated by the resource limitations imposed by the target device (Altera Stratix II EP2S180F1508C4) and real-time speed requirements, our current implementation can support filter kernel sizes $(N)$ from the list $\{5,7\}$ and $\{3,5\}$ for anisotropic diffusion filtering and median filtering, respectively. The corresponding kernel sizes for the embedded Gaussian filtering in the case of anisotropic diffusion filter supported by our architecture are $\{3,5\}$. Table 3.5 also lists the absolute and percentage logic resources consumed by the important modules in the architecture and the maximum operating frequency $\left(f_{\max }\right)$ at which these modules can run for a specific instantiation (choice of $N$ ). The percentage logic resources are reported in reference to the target device Altera Stratix II EP2S180F1508C4. The images used for this performance and logic consumption analysis were 8-bit images $(b=8)$. The choices for the value of $N$ for this analysis represent common kernel size choices and are listed in the fourth column of the table.

Table 3.6: Hardware requirements for the components of the linear systolic implementation of the 3D median filtering.

\begin{tabular}{cc}
\hline \hline Hardware component & Number required \\
\hline Processing elements & $b$ \\
Data registers & $b \times N^{3}$ \\
Mask select registers & $b \times N^{3}$ \\
Data pipeline registers & $(b-1) \times N^{3}$ \\
Line delay elements & $(b-2) \times(b-1) \times\left(N^{3}+1\right) / 2$ \\
\hline \hline
\end{tabular}




\subsubsection{Filtering Performance}

The 3D median filtering module and 3D anisotropic diffusion filtering module were synthesized for kernel sizes of $\{3,5\}$ and 7 , respectively, for filtering 8-bit images. The rest of the system, including the memory controller and input and output buffers, was parametrically synthesized to support the desired filtering operation and kernel size. The entire system was clocked at $200 \mathrm{MHz}$, which also corresponds to the filtering pipeline frequency (i.e., $T_{\text {proc }}=5 \mathrm{~ns}$ ). The image memories were also clocked at $200 \mathrm{MHz}\left(T_{\text {mem }}=5 \mathrm{~ns}\right)$. For this configuration, Table 3.7 reports the execution time for 3D anisotropic diffusion filtering and 3D median filtering as obtained during postsynthesis timing simulation of the entire system. The image sizes used for this measurement correspond to typical dimensions of intraprocedural images.

As indicated in Table 3.7, our implementation of 3D anisotropic diffusion filtering and 3D median filtering can easily achieve a processing rate of 46 frames per second (fps) for images of size $256 \times 256 \times 64$ voxels, which is a typical size of an intraprocedural volumetric CT scan. The corresponding processing rate for intraprocedural 3D ultrasound scan with typical dimensions of $128 \times 128 \times 128$ voxels

Table 3.7: Execution time of 3D anisotropic diffusion filtering and 3D median filtering.

\begin{tabular}{ccccc}
\hline Filter kernel & $\begin{array}{c}\text { Kernel } \\
\text { size } \\
\text { (N) }\end{array}$ & $\begin{array}{c}\text { Image size } \\
\text { (voxels) }\end{array}$ & $\begin{array}{c}\text { Execution } \\
\text { time } \\
\text { (ms) }\end{array}$ & $\begin{array}{c}\text { Voxel } \\
\text { processing rate } \\
\text { (MHz) }\end{array}$ \\
\hline 3D anisotropic & 7 & $128 \times 128 \times 128$ & 10.90 & 192 \\
diffusion filter & 7 & $256 \times 256 \times 64$ & 21.63 & 194 \\
\hline & 3 & $128 \times 128 \times 128$ & 10.75 & 195 \\
3D median & & $256 \times 256 \times 64$ & 21.44 & 196 \\
\cline { 2 - 5 } filter & 5 & $128 \times 128 \times 128$ & 10.82 & 194 \\
& & $256 \times 256 \times 64$ & 21.58 & 194 \\
\hline
\end{tabular}


is around 92 fps. For iterative operations such as anisotropic diffusion filtering or sequential filtering operations, this processing rate translates to $18 \mathrm{fps}$ with five iterations (or sequential operations) per frame, which is sufficient to meet the realtime needs of most IGIs.

Table 3.8 and Table 3.9 compare the execution speed of the presented architecture for 3D anisotropic diffusion filtering and 3D median filtering, respectively, against a corresponding software implementation and previously reported high-speed implementations using different computing platforms. The execution time has been normalized by the image dimensions for all implementations, and the performance is presented in terms of voxel processing rate to facilitate a fair comparison independent of image dimensions. The software implementation was developed using $\mathrm{C}++^{+}$, and its performance was measured on an Intel Xeon $3.6 \mathrm{GHz}$ workstation with 2 gigabytes of DDR2 $400 \mathrm{MHz}$ main memory. Although this architecture can support various kernel sizes for the filtering operations, for consistency the performance has been compared for a kernel size $(N)$ common to all implementations: $N=3$ for the median filtering and $N=7$ for anisotropic diffusion filtering.

Table 3.8: $\quad$ Performance comparison of the 3D anisotropic diffusion filtering kernel.

\begin{tabular}{ccccc}
\hline \hline Implementation & Platform & $\begin{array}{c}\text { Filter } \\
\text { kernel }\end{array}$ & $\begin{array}{c}\text { Voxel } \\
\text { processing rate } \\
\text { (MHz) }\end{array}$ & Speedup \\
\hline $\begin{array}{c}\text { Software (C++) } \\
\text { Bruhn et al. }\end{array}$ & Xeon workstation & 3D & 0.92 & 208 \\
$\quad$ 256-processor cluster & 3D & 105 & 1.83 \\
$\begin{array}{c}\text { Tabik et al. [101] } \\
\text { Dandekar } \\
\text { et al. [144] }\end{array}$ & 16-processor cluster & 3D & 5.66 & 33.9 \\
\hline \hline
\end{tabular}


As indicated by Table 3.8, our implementation of 3D anisotropic diffusion filtering provides more than two orders of magnitude speedup over the software implementation using a single workstation. Moreover, the performance of the current architecture represents an improvement over a corresponding implementation using a 256-processor computing cluster reported previously [100]. Our work presented a novel FPGA-based implementation of 3D anisotropic diffusion filtering. Salient features of this filtering module are an embedded Gaussian filtering implementation that minimizes the number of multipliers and a pipelined design that allows throughput of one output voxel per clock cycle. This filtering module offers the flexibility to support several anisotropic diffusion techniques previously reported in the literature. For example, the multiscale approach proposed by Whitaker and Pizer [82] can be implemented by changing the embedded Gaussian filter coefficients at the end of each iteration, and a time-dependent diffusion function [145] can be implemented by reprogramming the values in the diffusion function LUT. One limitation of this filtering module is the limit on the size of the embedded Gaussian filter kernel; implementing Gaussian kernels larger than 7 would result in prohibitively high hardware requirements. Such large kernels, however, are uncommon in most applications. Although this architecture performs some of the arithmetic functions using fixed-point representation, the variation in the output intensity values is only a small fraction of the intensity range, and these variations are unlikely to affect the accuracy and precision of IGIs.

Table 3.9 compares the performance of the FPGA-based 3D median filtering operation described in this work with previously reported high-speed 
Table 3.9: $\quad$ Performance comparison of the 3D median filtering kernel.

\begin{tabular}{ccccc}
\hline \hline Implementation & Platform & $\begin{array}{c}\text { Filter } \\
\text { kernel }\end{array}$ & $\begin{array}{c}\text { Voxel processing } \\
\text { rate (MHz) }\end{array}$ & Speedup \\
\hline Software (C++) & Xeon workstation & 3D & 2.63 & 74 \\
Viola et al. [136] & GPU & 3D & 0.76 & 257 \\
Gallegos-Funes and & DSP & 2D & 4.5 & 43 \\
Ponomaryov [146] & & & & \\
Jiang and Crookes & FPGA & 3D & 50 & 3.9 \\
[137] & FPGA & 3D & 195 & - \\
Dandekar et al. [144] & & &
\end{tabular}

implementations. The present implementation provides more than an order of magnitude speedup over software- and GPU-based 3D implementations and DSPbased 2D implementation. Jiang and Crookes [137] recently reported an FPGA-based 3D implementation that is capable of achieving a voxel processing rate of $50 \mathrm{MHz}$. That design, however, was based on a partial sorting technique and cannot be easily extended to kernel sizes beyond 3. Our implementation, in contrast, achieved a superior voxel processing rate and is sufficiently compact to allow implementation of kernel sizes up to 7, which is sufficient for most common image processing tasks. The logic resources required by the described systolic array-based median filter indeed scale up as kernel sizes get larger; but as modern FPGAs become denser and offer improved logic capacity, this requirement is still a small percentage of the total available resources (see Table 3.5).

\subsection{Summary}

This chapter presented an FPGA-based architecture for real-time preprocessing of volumetric images acquired during IGIs. The developed architecture enables 3D anisotropic diffusion filtering and 3D median filtering of intraprocedural 
images at the rate of $50 \mathrm{fps}$, which is faster than current acquisition speeds of most imaging modalities. The solution presented offers real-time performance, is compact and accurate, and, hence, suitable for integration into IGI workflow. As IGI applications become increasingly popular, intraprocedural imaging modalities continue to offer wider coverage and higher imaging speed. Thus, there is a corresponding need for real-time processing of these images. The real-time performance of our design along with the throughput of one voxel per cycle can cater to these $4 \mathrm{D}(3 \mathrm{D}+$ time) image processing needs. 


\section{Chapter 4: Hardware-Accelerated Deformable Image}

\section{Registration}

Intensity-based deformable image registration plays a critical role in many diagnostic and interventional applications requiring image combination. Despite the advantages (such as accuracy, automation, and retrospective nature) of this approach, these algorithms find limited use in clinical applications due to their computational complexity. This chapter presents a novel FPGA-based architecture for accelerated implementation of MI-based deformable registration. This architecture is capable of reducing the execution time of MI-based deformable registration from hours to a few minutes. First, we describe the registration algorithm that is being accelerated. Next, we present the architecture for its high-speed implementation. Finally, we characterize the execution performance of this architecture and provide qualitative validation results. The optimization of this architecture for accuracy and hardware resources is presented in Chapter 5. The quantitative validation and the clinical applicability of this architecture are presented later, in Chapter 6.

\subsection{Motivation}

Combining complementary information from intraprocedural and preprocedural images is a fundamental need in IGI applications. These images, however, are acquired at different times and using different imaging scanners and protocols and as a result are usually misaligned. Therefore, they need to be registered (or aligned) for a meaningful combination and fusion of the information they contain. Deformable image registration techniques can compensate for both local deformation 
and large-scale tissue motion and are the ideal solution for achieving the aforementioned image registration. Some studies, in particular, have independently underlined the importance of deformable registration and/or soft tissue modeling for IGIs [18, 19]. However, despite their advantages, deformable registration algorithms are seldom used in current clinical practice. The large number of degrees of freedom that these algorithms employ makes them extremely computationally intensive. On a modern workstation most deformable registration algorithms can take several hours, which is clearly unacceptable for IGIs requiring on-demand performance. As a result, most earlier reported techniques for aligning preprocedural and intraprocedural images employ rigid body approximation, which is often not valid because of underlying nonrigid tissue deformation. In addition, some of these techniques are not retrospective (i.e. they require some advanced planning at the time of preprocedural imaging), which further limits their applicability.

Mutual information (MI)-based deformable registration has been shown to be effective in multimodality image registration because of the robustness of the similarity measure [69]. Moreover, MI-based image registration is automatic and completely retrospective because it uses image intensities to achieve the alignment. Walimbe and Shekhar [60, 67] have earlier reported an MI-based deformable registration algorithm that utilizes volume subdivision. Hierarchical volume subdivision-based image registration techniques are inherently faster than most other deformable registration techniques and are more amenable to hardware acceleration. This algorithm has been used and rigorously validated in the context of PET-CT registration [66]. This clinical validation has demonstrated the registration accuracy 
of the aforementioned algorithm to be comparable to a group of clinical experts and the mean registration accuracy for the abdominal region to be superior to an earlier reported free-form deformation (FFD)-based technique [147]. This algorithm is theoretically general and has been shown to be effective for various applications employing multimodal deformable registration [66, 77, 148-151]. Although computationally efficient, software implementation of this algorithm can take several hours, which is still slow for direct integration into the IGI workflow. It is, therefore, necessary to accelerate this algorithm and reduce the processing time to the order of minutes and ultimately to seconds for its assimilation into clinical workflow. Although, accelerated implementations of MI-based deformable registration algorithms using very large multiprocessor clusters have been proposed earlier [57, 115, 117, 118], their per-node performance does not compare favorably with our implementation. Furthermore, these solutions may not be cost effective and are unlikely to be suitable for clinical deployment.

The chapter presents a novel field-programmable gate array (FPGA)-based accelerated implementation of the aforementioned deformable registration algorithm, specially geared toward improving target delineation during image-guided interventions. The reported solution provides a speedup of about 40 for MI calculation, thus reducing the deformable registration time from hours to minutes. In Chapter 6, we demonstrate fast and successful registration of intraprocedural abdominal CT scans with preprocedural CT and PET scans using the developed architecture. We further demonstrate that the registration accuracy of the hardware implementation is comparable with that using a software implementation and is on 
the order of a few millimeters. This registration accuracy coupled with the execution speed and compact implementation of the reported solution makes it suitable for integration in the IGI workflow.

\subsection{Algorithm for Deformable Image Registration}

Hierarchical volume subdivision-based deformable image registration techniques are inherently faster than most intensity-based deformable registration techniques (e.g., FFD-based techniques) and are more amenable to acceleration through hardware implementation. 3D image registration using volume subdivision has been proposed earlier, but the earlier implementations were limited to a locally translation-based model. Walimbe and Shekhar [60,67] enhanced this model by incorporating local rotations and reported a quaternion-based scheme for interpolating multiple 3D rigid-body transformations for deformable registration using the volume subdivision approach. For a pair of images, one treated as reference and the other as floating, this algorithm performs deformable registration using a series of hierarchical, locally rigid-body registrations. The six-parameter rigid registration at

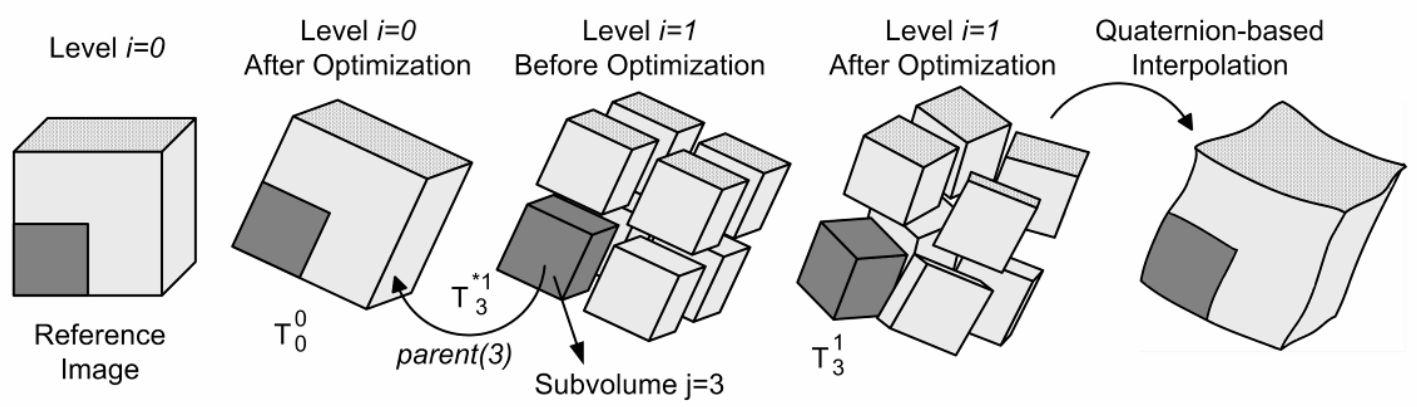

Figure 4.1: Pictorial representation of hierarchical volume subdivision-based deformable image registration and associated notation. 
each level is optimized by maximizing the MI between the reference and floating images (RI and FI, respectively). This hierarchical registration scheme is shown in Figure 4.1.

The initial optimal rigid alignment (at the root level) between RI and FI can be represented using a transformation matrix $T_{0}^{0}$ (where $T_{j}^{i}$ represents the cumulative optimal transformation at level $i$ for subvolume $j$ ). Next, the algorithm uses a hierarchical octree-based subdivision scheme. At each subdivision level $i$, the RI is divided into $8^{i}$ subvolumes, numbered from 0 to $8^{i}-1$. Each of these subvolumes is then individually registered with the FI, under transformation range constraints derived from the transformation of its parent subvolume at the earlier level $T_{\text {parent }(j)}^{i-1}$. The notation parent $(j)$ refers to the subvolume at the previous subdivision level $i-1$, which contains the current subvolume $j$. For example, at the root level $(i=0)$, there is a single subvolume (entire image) numbered $j=0$. After one level of subdivision ( $i=1$ ), there will be eight subvolumes numbered from $j=0$ to $j=7$. At level $i=1$, parent(3) refers to subvolume numbered 0 at level $i=0$, because it contains subvolume $j=3$ at the current level $(i=1)$ of subdivision (see Figure 4.1). The optimal alignment of the subvolume $j$ within the FI is also determined by maximizing MI under a six-parameter rigid-body transformation model.

Volume subdivision and subvolume registration continue until the voxel count for an individual subvolume remains above a predefined limit (usually $16^{3}$ ) to yield a statistically significant similarity measure. Thus, this algorithm achieves hierarchical refinement of the localized matching between RI and FI. The final cumulative non- 
rigid alignment between the image pairs is computed by quaternion-based direct interpolation of the individual subvolume transformations at the final subdivision level.

\subsubsection{Calculating MI for a Subvolume}

Registration of a subvolume during the hierarchical refinement process is based on maximization of the MI, which is a statistical measure. With progressive subdivision, subvolumes at every level become increasingly smaller. The mutual histogram (MH) corresponding to an individual subvolume becomes sparse, thus rendering MI unreliable. The aforementioned algorithm addresses this issue by using the $\mathrm{MH}$ of the entire image (all the subvolumes) to calculate MI during the registration of a subvolume. The contribution of the current subvolume $k$ at level $i$ to the $\mathrm{MH}$ is computed under the current candidate transformation $T_{k}^{*_{i}}\left(T^{*}\right.$ denotes a candidate transformation during the optimization process). The contribution to the $\mathrm{MH}$ from the rest of the subvolumes remains constant during this registration process and is derived from their parent subvolumes. Thus, MI is computed over the entire image with local variations corresponding to the subvolume under optimization. Equations (4.1)-(4.3) summarize this process. The function Accumulate(T), contributes to the $\mathrm{MH}$ using the voxels in a given subvolume $k$, using the mapping provided by the given transformation $T$. The detailed description of this deformable registration algorithm can be found in [60].

$$
\begin{gathered}
M H_{\text {Total }_{k}}^{i}=M H_{\text {Subvolume }_{k}}^{i}+M H_{\text {Rest }_{k}}^{i} \\
M H_{\text {Subvolume }_{k}}^{i}=\text { Accumulate }\left(T_{j}^{* i}\right)
\end{gathered}
$$




$$
M H_{\text {Rest }_{k}}^{i}=\operatorname{Accumulate}\left(T_{\text {parent(j) }}^{i-1}\right)
$$

\subsection{Acceleration Approach}

The aforementioned algorithm uses MI as a measure of image similarity. MI is an intensity-based similarity measure and calculation of MI requires processing of all the voxels in the RI. Registration through maximization of MI attempts to find the transformation that best aligns an FI with an RI. This MI-based registration typically requires thousands of iterations (MI evaluations), depending on the image complexity and the degree of initial misalignment between the images. Repeated MI computation, which requires accessing both the images (RI and FI), is memory access intensive, and in particular, the memory access in the FI is completely governed by the transformation applied. This operation, therefore, does not benefit from the cachebased memory architectures present in most modern PCs (the caches are too small to fit 3D images). Because memory speed has not evolved at the same rate as microprocessor speed, introduction of faster microprocessors is not expected to significantly speed up image registration. Thus, a factor limiting the performance of software implementations is calculating MI for different candidate transformations. Castro-Pareja et al. [139] have shown that, for typical medical images, accumulating

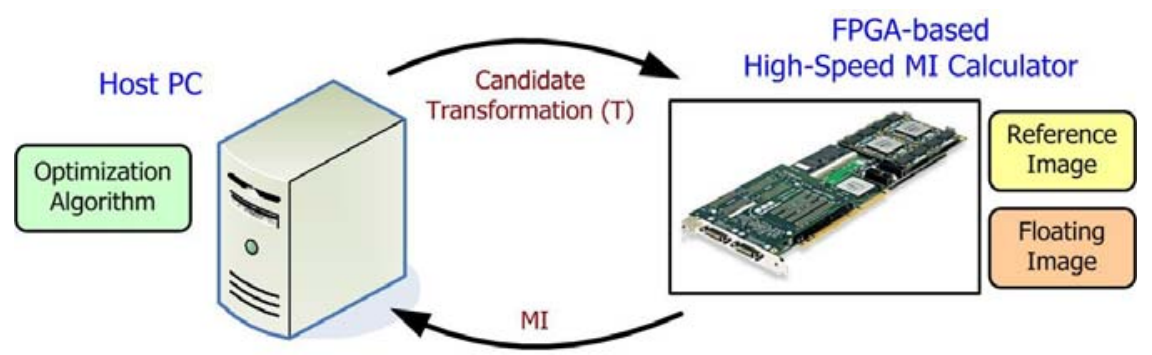

Figure 4.2: $\quad$ Pictorial representation of the acceleration approach. 
the MH and calculation of MI can take up to 99\% of the total image registration time in software. Our efforts for acceleration of this algorithm, consequently, are targeted toward optimized and pipelined implementation of $\mathrm{MH}$ accumulation and $\mathrm{MI}$ calculation. This approach is pictorially represented in Figure 4.2.

In general, the execution time $T$ required by a pipelined implementation (with n-stages) of an operation is given as:

$$
T=t \cdot\left(\frac{N}{m}+n\right)
$$

where $t$ is the latency of each stage, $N$ is the amount of data to be processed, and $m$ is the number of data units processed in parallel at each stage. In the case of MI calculation, $N$ represents the number of RI voxels to be processed. For typical volumetric images (which are usually larger than $128^{3}$ ) it can be assumed that $\frac{N}{m} \gg n$, thus the main parameters that control the MI calculation time are then $t$ and $m$. Supporting $m>1$ requires a superscalar architecture and multiple processing pipelines with individual image memory access, which is not practical. Therefore, to provide maximum pipeline performance, our architecture focuses on reducing $t$, the latency of each pipeline stage. The lower bound on $t$ is the period of the system clock and achieving this bound means that all the pipeline stages (including image memory access) complete their operations in one clock cycle. The following section describes an architecture geared toward meeting this goal for accelerated calculation of MI. 


\subsection{Architecture}

MI-based image registration can be thought of as an optimization problem of finding the best alignment between two images. During the execution of the algorithm, the optimization process is executed from the host workstation. This host provides a candidate transformation, while the presented FPGA-based solution applies it to the images and performs corresponding MI computation. The computed MI value is then further used by the host to update the candidate transformation and eventually find the optimal deformable alignment between the RI and the FI. This workflow is indicated in Figure 4.2. The top-level block diagram of the FPGA-based architecture for accelerated implementation of volume subdivision-based image registration is shown in Figure 4.3. The important modules in this architecture are described below.

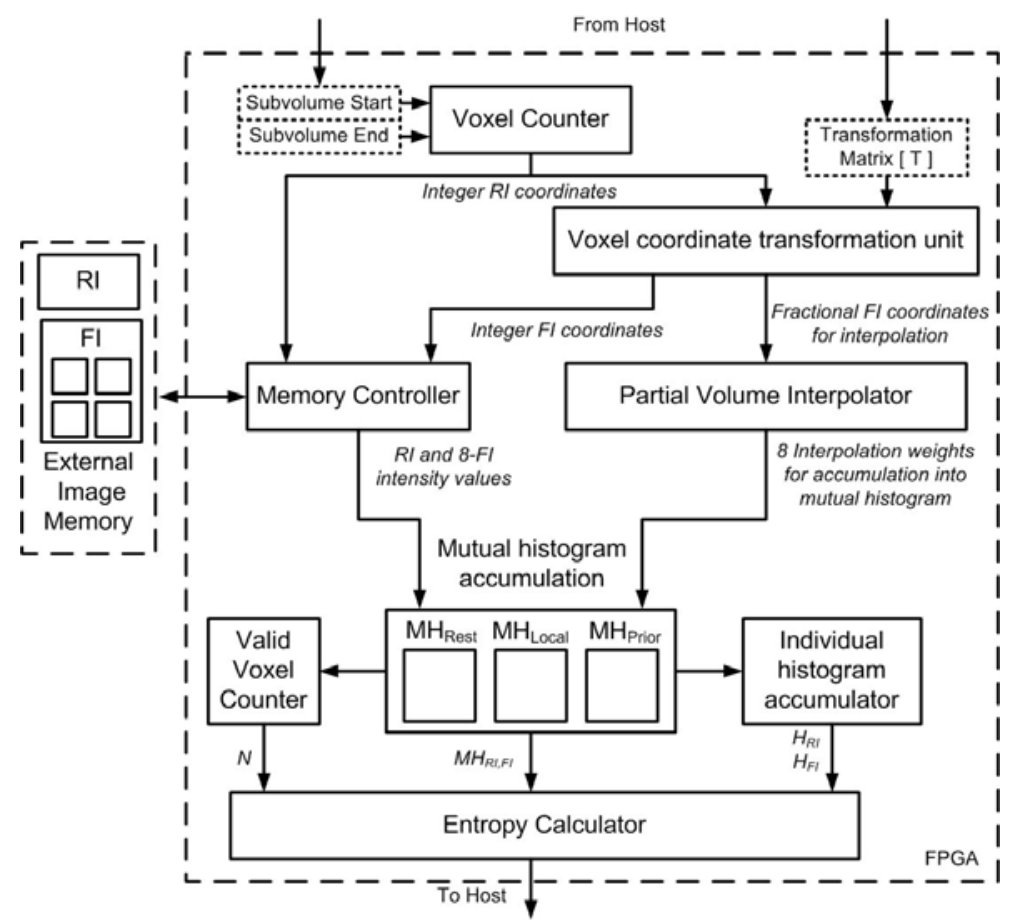

Figure 4.3: Top-level block diagram of the architecture for accelerated implementation of deformable image registration. 


\subsubsection{Voxel Counter}

Calculation of MI requires processing every voxel in the RI. This can be achieved by sequentially cycling though all the voxels in the RI. In addition, because the implemented algorithm is based on volume-subdivision, RI voxels within a 3D neighborhood corresponding to an individual subvolume must also be processed sequentially. This is implemented as follows: the host programs the FPGA-based MI calculator with subvolume start and end addresses. In case of the entire image (as required for rigid registration), the start address is $(0,0,0)$ whereas the end address is equal to the dimensions of the RI. When a subvolume needs to be processed (for example, during subvolume optimization after image subdivision), the start and end addresses programmed by the host correspond to that of the subvolume. The voxel counter sequentially computes the addresses corresponding to all voxels within a given neighborhood range (a subvolume, for example) in $z-y-x$ order. This is implemented using three synchronized counters, one for each dimension. A functional diagram of this module is presented in Figure 4.4. Through pipelined implementation, this module is capable of generating address for one RI voxel per clock cycle. This

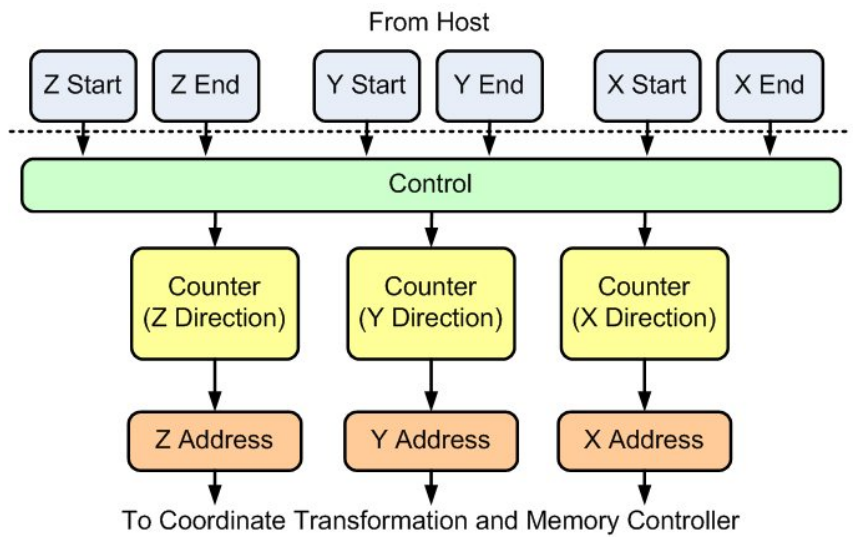

Figure 4.4: Functional block diagram of voxel counter. 
module operates in two modes: in the reference image processing mode the address generated is used to fetch the RI image voxels from the external image memory, whereas in the floating image processing mode the RI address generated is transformed to the floating image space for further processing.

\subsubsection{Coordinate Transformation}

The initial step in MI calculation involves applying a candidate transformation $\left(T_{j}^{* i}\right)$, to each voxel coordinate $\left(\vec{v}_{r}\right)$ in a subvolume $j$ of the RI to find the corresponding voxel coordinates in the FI (represented using $\vec{v}_{f}$ ). This is mathematically represented as shown in (4.5). Because the algorithm is linear at every subvolume, this is implemented using the six-parameter rigid transformation model.

$$
\vec{v}_{f}=T_{j}^{* i} \cdot \vec{v}_{r}
$$

An interesting aspect of this coordinate transformation and subsequent operations is that they involve operations in both real (millimeter) space as well as voxel address (image index) space. For example, the transformation (translations and rotations) is defined in the real space, whereas the voxels to be fetched from RI and FI are identified in voxel address space. Thus, there is a need to convert between these address spaces during the calculation of MI. This conversion can be performed by utilizing the voxel size information associated with each image. To circumvent the need for performing this conversion in our FPGA-based architecture, we appropriately scale the transformation matrix (in millimeter space) and represent it using a mathematically equivalent matrix in voxel address space. This conversion is performed in software by the host just prior to MI calculation. This matrix is then fed 
to the FPGA-based MI calculator and the MI calculator performs all its operations in voxel address space. This converted transformation can be represented as:

$$
\left[\begin{array}{c}
p \\
q \\
r
\end{array}\right]=\left[\begin{array}{cccc}
r_{x x} \cdot \frac{v r_{x}}{v f_{x}} & r_{x y} \cdot \frac{v r_{x}}{v f_{y}} & r_{x z} \cdot \frac{v r_{x}}{v f_{z}} \\
r_{y x} \cdot \frac{v r_{y}}{v f_{x}} & r_{y y} \cdot \frac{v r_{y}}{v f_{y}} & r_{y z} \cdot \frac{v r_{y}}{v f_{z}} \\
r_{z x} \cdot \frac{v r_{z}}{v f_{x}} & r_{z y} \cdot \frac{v r_{z}}{v f_{y}} & r_{z z} \cdot \frac{v r_{z}}{v f_{z}}
\end{array}\right] \cdot\left[\begin{array}{c}
i-R I_{c x} \\
j-R I_{c y} \\
k-R I_{c z}
\end{array}\right]+\left[\begin{array}{c}
\frac{T_{x}}{v f_{x}} \\
\frac{T_{y}}{v f_{y}} \\
\frac{T_{z}}{v f_{z}}
\end{array}\right]+\left[\begin{array}{c}
F I_{c x} \\
F I_{c y} \\
F I_{c z}
\end{array}\right] .
$$

In this equation, the tuple (i, j, k) represents the address of a voxel in RI space. This address is calculated by the voxel counter. The $r_{i j}$ s represent the components of the rotation matrix, whereas $T_{i} \mathrm{~s}$ represent the components of the translation vector. $v r_{i} \mathrm{~s}$ and $v f_{i}$ s represent the voxel sizes (in millimeters) of the reference image and the floating image, respectively. $\left(R I_{c x}, R I_{c y}, R I_{c z}\right)$ and $\left(F I_{c x}, F I_{c y}, F I_{c z}\right)$ represent the centers of the reference image and floating image in voxel address space, respectively.

This transformation model is represented using a $3 \times 3$ rotation matrix and a

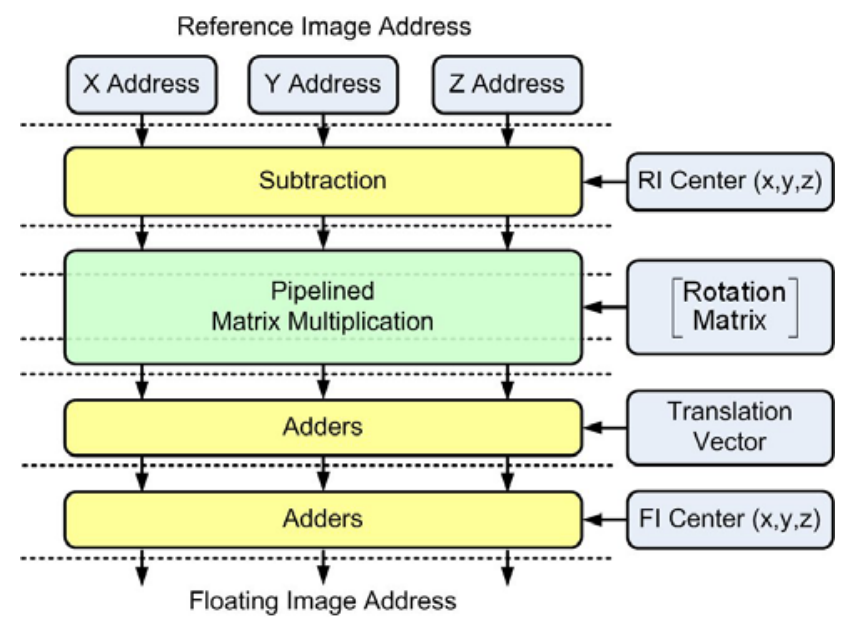

Figure 4.5: Functional block diagram of coordinate transformation unit. 
$3 \times 1$ translation vector. The host calculates these transformation components by appropriate scaling (as described above) of the current candidate transformation provided by the optimization routine and sends it to the MI calculator. Fixed-point representation is used to store the individual elements of these transformation components. The coordinate transformation is accomplished by simple arithmetic computations such as additions and multiplications as illustrated in Figure 4.5. The pipelined implementation of this module allows transformation of one RI voxel address per clock cycle.

\subsubsection{Partial Volume Interpolation}

The coordinates mapped in the FI space $\left(\vec{v}_{f}\right)$ do not normally coincide with a grid point (integer location), thus requiring interpolation. This scenario is illustrated in Figure 4.6 (a). In this figure, for simplicity, we have presented a 2-dimensional case; but similar scenario presents itself in three dimensions as well. The calculation of interpolation weights associated with the neighborhood (again, for a 2-dimensional case), in which the mapped coordinate lands, is illustrated in Figure 4.6 (b). In comparison, a 3-dimensional case will have 8-interpolation weights. Nearestneighbor, linear, and partial volume (PV) interpolation schemes have been

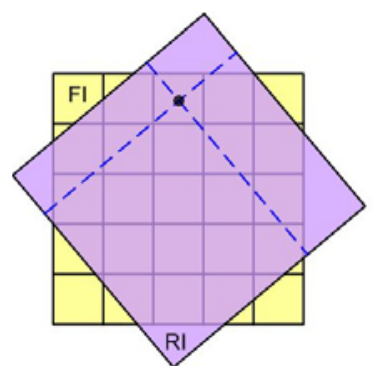

(a)

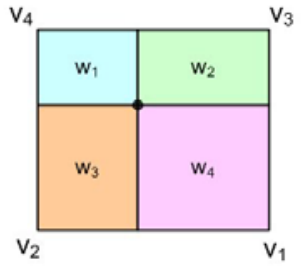

(b)

\begin{tabular}{|c|c|c|}
\hline $\begin{array}{c}\text { Interpolation } \\
\text { Scheme }\end{array}$ & Output Intensity(s) & Output Weight(s) \\
\hline Nearest & $\mathrm{v} 4$ & 1 \\
\hline Linear & $\begin{array}{c}\mathrm{v} 1 . \mathrm{w} 1+\sqrt{2} \cdot \mathrm{w} 2+ \\
\sqrt{3} \cdot \mathrm{w} 3+\mathrm{v} 4 . \mathrm{w} 4\end{array}$ & 1 \\
\hline Partial Volume & $(\mathrm{v} 1, \sqrt{ } 2, \mathrm{v} 3, \mathrm{v} 4)$ & $(\mathrm{w} 1, \mathrm{w} 2, \mathrm{w} 3, \mathrm{w} 4)$ \\
\hline
\end{tabular}

(c)

Figure 4.6: Fundamentals of interpolation schemes. 
traditionally used for this purpose. Mathematical formulation for calculation of the interpolation weights using these techniques is presented in Figure 4.6 (c). The primary difference between PV and linear interpolation is that, PV interpolation does not consolidate the weights and associated intensity values through weightedsumming. Instead, it preserves the intensity-interpolation weight pairs and updates the mutual histogram $(\mathrm{MH})$ at corresponding 8-locations (for a $3 \mathrm{D}$ case). PV interpolation scheme increases the memory access requirement of the $\mathrm{MH}$ accumulation operation, but is capable of providing smooth changes in the $\mathrm{MH}$ values (and associated smoother optimization curve) with small changes in transformation [69].

Consequently, our architecture implements PV interpolation as the choice of interpolation scheme. $\vec{v}_{f}$, in general, will have both fractional and integer components and will land within an FI neighborhood of size $2 \times 2 \times 2$. The interpolation weights required for the PV interpolation are calculated using the fractional components of $\vec{v}_{f}$. These weights can be calculated as follows:

$$
\begin{aligned}
& W_{0}=(1-x f) \cdot(1-y f) \cdot(1-z f) \\
& W_{1}=(1-x f) \cdot(1-y f) \cdot z f \\
& W_{2}=(1-x f) \cdot y f \cdot(1-z f) \\
& W_{3}=(1-x f) \cdot y f \cdot z f \\
& W_{4}=x f \cdot(1-y f) \cdot(1-z f) \\
& W_{5}=x f \cdot(1-y f) \cdot z f \\
& W_{6}=x f \cdot y f \cdot(1-z f) \\
& W_{7}=x f \cdot y f \cdot z f
\end{aligned}
$$

In this equation, the tuple $(x f, y f, z f)$ correspond to fractional components of $\vec{v}_{f}$ in $\mathrm{x}, \mathrm{y}$, and $\mathrm{z}$ dimensions respectively. This interpolation scheme is implemented in this architecture by implementing the above formulation using simple arithmetic 
computations such as additions and multiplications as illustrated in Figure 4.7. The pipelined implementation of these operations allows processing of one transformed RI voxel per clock cycle. Fixed-point arithmetic is used to perform these operations. The corresponding floating voxel intensities are fetched by the image controller in parallel using the integer component of $\vec{v}_{f}$. The image controller also fetches the voxel intensity corresponding to $\vec{v}_{r}$. The MH, then must to be updated for each pair of reference and floating voxel intensities (8 in all), using the corresponding weights computed by the PV interpolator.

\subsubsection{Image Memory Access}

The dimensions of typical 3D medical images are in the range of $128 \times 128 \times$ 128 to $512 \times 512 \times 512$ voxels. Because intensity-based image registration typically

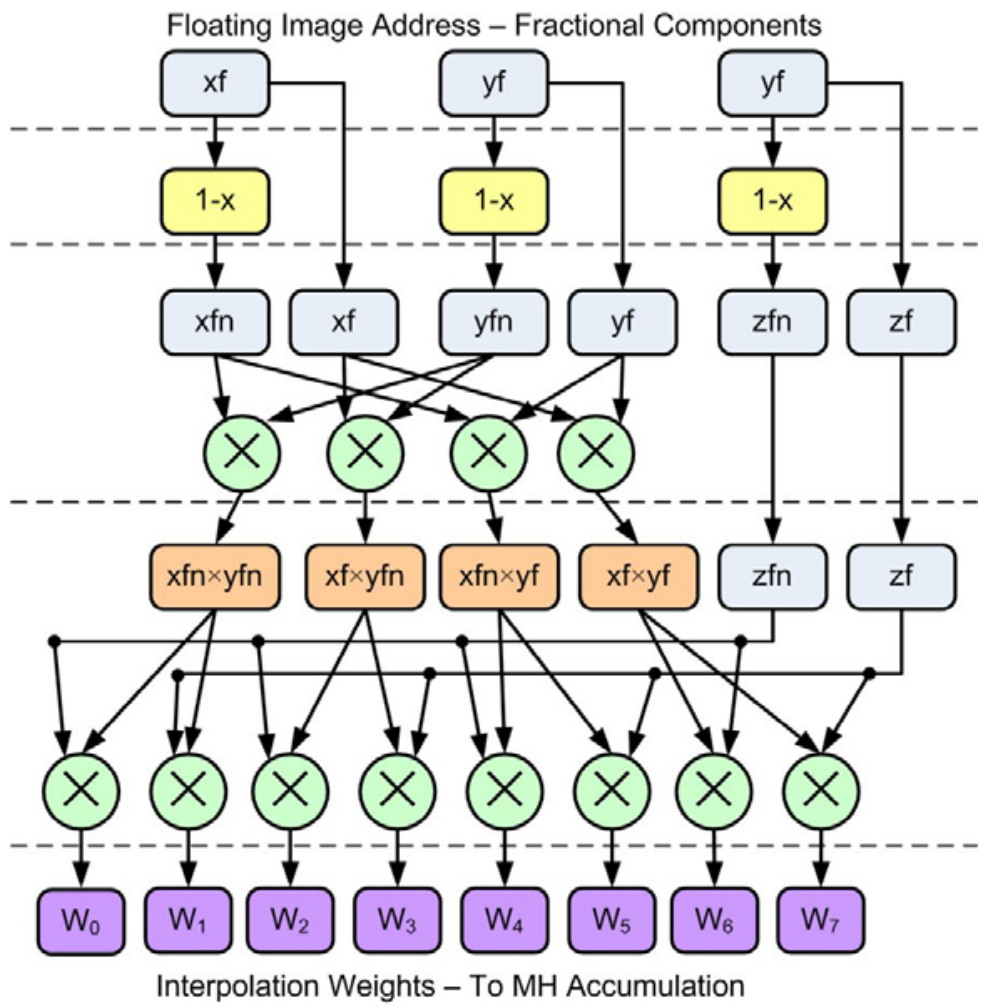

Figure 4.7: $\quad$ Functional block diagram of partial volume interpolation unit. 
operates on images with 8-bit intensity values per voxel, this corresponds to image sizes ranging from 2 to 128 Megabytes. In comparison, the internal, high-speed memory provided by modern FPGAs is limited to 16 Megabits (or equivalent to 2 Megabytes). Consequently, the images to be registered can not be stored using the memory that is internal to the FPGA and external storage must be employed for this purpose.

Because of the size of the medical images, use of static random access memory (SRAM) modules (which are fast, but offer poor density and capacity) is not very efficient. Dynamic random access memory (DRAM) modules, in comparison, offer the necessary density to store the large images that are typically encountered in medical imaging. Although, these memories do not allow random accesses throughout the entire memory module with a uniform latency, they support burstmode accesses that allow efficient random accesses within a single row of the memory module. Our architecture takes advantage of this feature and uses external DRAM memories to store the images to be registered (in other words, the RI and FI).

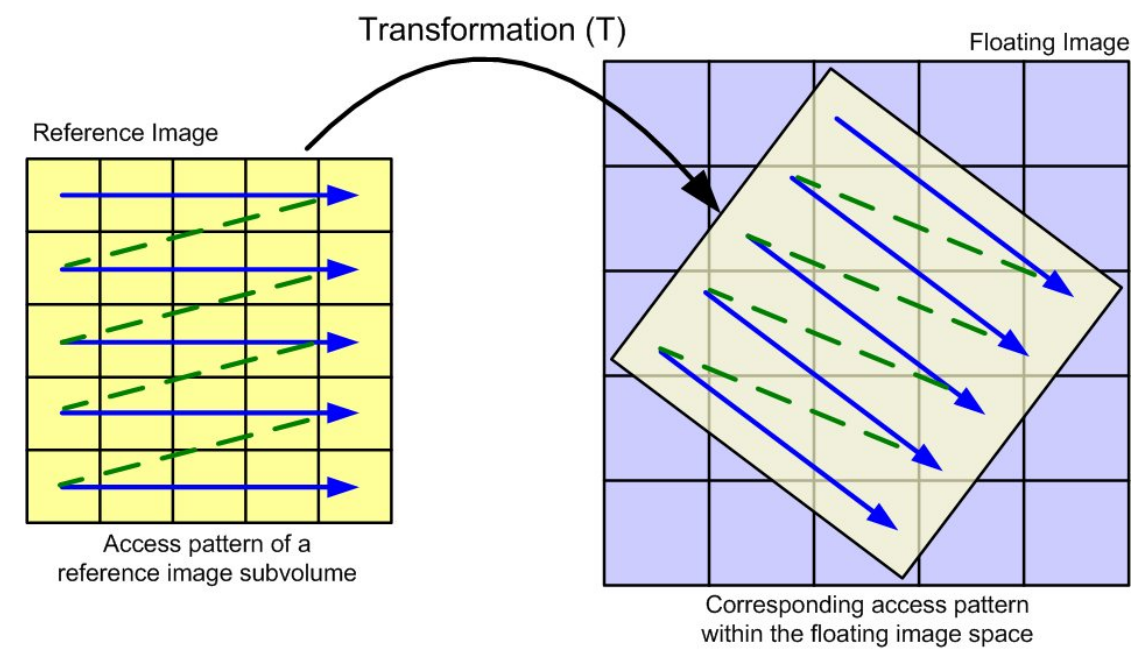

Figure 4.8: Voxel access patterns of the reference and floating images encountered during image registration. 
During calculation of MI for a given voxel location in the RI, the reference image memory must be accessed only once, whereas a complete $2 \times 2 \times 2$ neighborhood (as required for PV interpolation) must be fetched from the floating image memory. Thus, there is a disparity between the memory access needs for these two images. The following subsections briefly describe the access requirements for both these images and present the strategies we adopted to meet those requirements.

\subsubsection{Reference Image}

An example of memory access pattern for the RI during image registration is illustrated in Figure 4.8. Between the two images, the RI has more relaxed access requirements, because it is accessed in a sequential manner (in $z-y-x$ order) and only one voxel must be fetched from the RI for a given RI voxel address. This kind of access benefits from burst accesses and memory caching techniques. Furthermore, the implemented algorithm is based on volume-subdivision and requires traversing through the RI on a subvolume basis. This means that the voxels that belong to a subvolume need to be consecutively accessed.

An interesting feature of modern DRAMs is that they are logically partitioned into rows and columns. As a result, these memories incur additional latency of several clock cycles when switching between different rows of the memory. Accessing different columns within a given row, however, is efficiently supported through burst accesses and can be performed within one clock cycle. Our architecture takes advantage of this feature and stores the RI in such a way that each subvolume is aligned with a row of the DRAM memory. This memory organization is illustrated in Figure 4.9. This allows efficient access to the voxels that belong to a subvolume. 


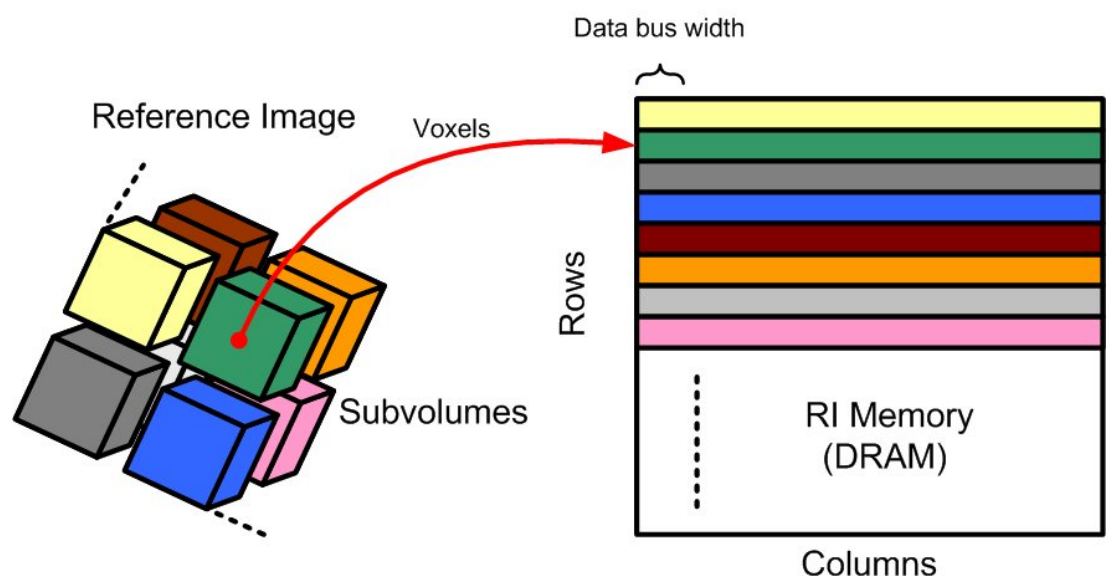

Figure 4.9: Organization of the reference image memory.

For the architecture presented, both the RI and FI are stored in separate logical partitions of the same DRAM module. However, during MI calculation both these memories must be accessed simultaneously over a single bus. This can lead to memory access conflicts and result into poor performance. To address this issue, our architecture uses internal memory of the FPGA to cache a sub-block of RI voxels. Thus, during the processing of that block of RI voxels, the image controller has parallel access to both RI and FI voxels. The RI voxels are fetched from the internal FPGA memory (with access time of 1 clock cycle), whereas the FI voxels are fetched directly from the external memory.

\subsubsection{Floating Image}

An example of memory access pattern for the FI during image registration is illustrated in Figure 4.8. The FI has much higher memory access requirements when compared to the RI. There are two primary reasons for this: 
1. For every RI voxel address to be processed, 8 FI voxels (corresponding to a $2 \times 2 \times 2$ neighborhood) must be fetched. This neighborhood is then further used for performing PV interpolation and $\mathrm{MH}$ accumulation.

2. Although, the RI is accessed in a sequential manner, the corresponding access pattern in the FI is completely governed by the transformation $\left(T_{j}^{*_{i}}\right)$ that is currently being applied. For example, in Figure 4.8 the access pattern in FI is not aligned with the natural dimensions $(x, y$, or $z)$ of the image.

The first aspect of this memory access requirement is similar to that encountered in the context of volume rendering. In the graphics domain, this problem has been solved by developing techniques that allow parallel access to the entire $2 \times 2 \times 2$ voxel neighborhood. One way to provide this parallel neighborhood access is through the use of cubic addressing [140]. Cubic addressing employs eight memory modules that are accessed in parallel at different addresses, through arithmetic manipulation of the corresponding single "neighborhood address". These parallel memory modules are thus capable of providing the entire 3D neighborhood in one

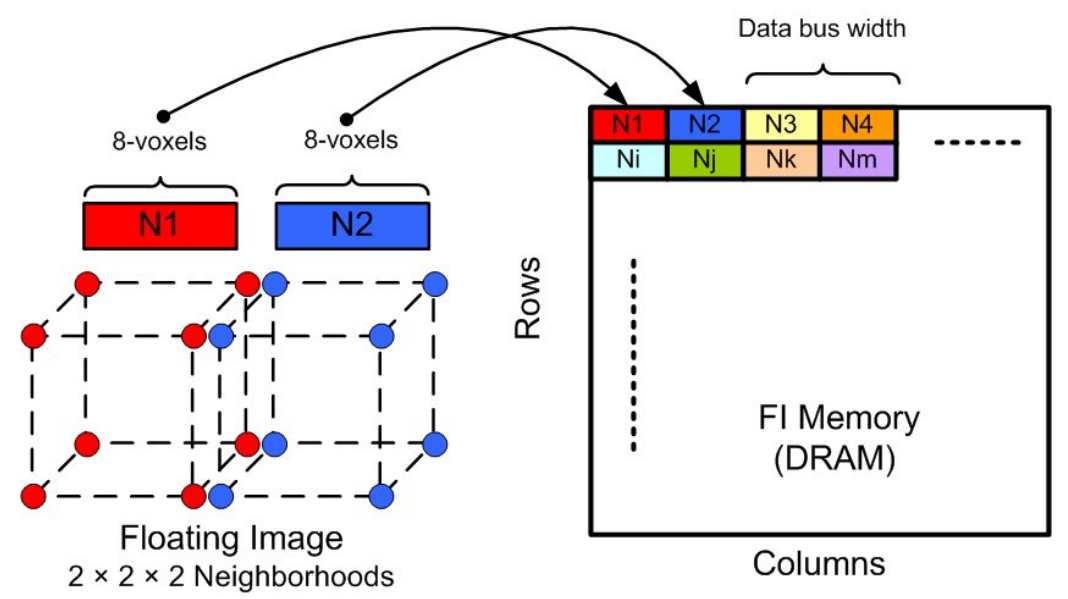

Figure 4.10: Organization of the floating image memory. 
clock cycle. This approach, however, requires custom addressing of the memory modules. Castro-Pareja et al. [139] have reported a method to implement cubic addressing using standard DRAMs, by storing multiple copies of the image. This approach does not require special addressing and take advantage of burst-mode accesses provided by DRAMs. The presented architecture implements a similar scheme by storing eight copies of the FI. This scheme is illustrated in Figure 4.10. The FI voxels are arranged sequentially such that, performing a size 2 burst fetches two adjacent $2 \times 2$ neighborhood planes, thus making the entire neighborhood available simultaneously. Although this approach increases the image storage, it enables accessing the entire 3D neighborhood in one clock cycle. Further, the density of DRAM chips allows storing multiple copies of the FI.

The second aspect of the FI memory access requirement is addressed through the following strategy: we group the neighborhoods that belong to an image block (within the FI), and assign those neighborhoods to a single row in the DRAM module. Thus, as long as the transformed address location $\left(\vec{v}_{f}\right)$ stays within a FI subvolume, the corresponding neighborhoods can be fetched within one clock cycle. Because, the RI is traversed one subvolume at a time, it is highly likely that the transformed location of that subvolume is confined to one or few FI subvolumes. Our architecture takes advantage of this spatial locality of reference.

\subsubsection{Updating Mutual Histogram}

For a given RI voxel $R V$, there are eight intensity pairs $\left(R V, F V_{0}: F V_{7}\right)$ and corresponding interpolation weights. Because the MH must be updated (read- 


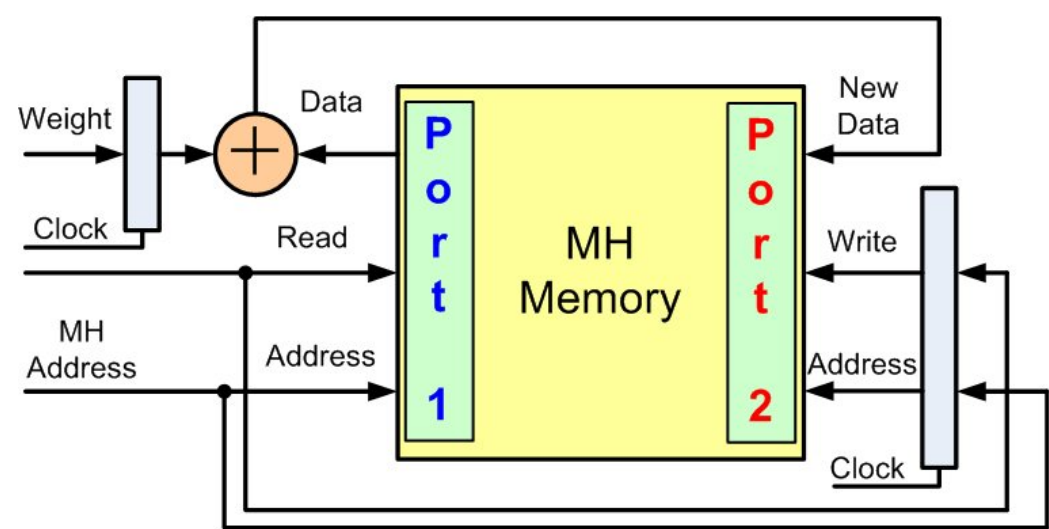

Figure 4.11: Pipelined implementation of $\mathrm{MH}$ accumulation using dual port memory.

modify-write) at these eight locations, this amounts to 16 accesses to $\mathrm{MH}$ memory for each RI voxel. This means that $\mathrm{MH}$ memory has more stringent access requirements when compared with the floating image memory. Moreover, the locations that need to be accessed within the MH for a given RI voxel, may not have any relationship with each other (such as a neighborhood-relation in the context of FI memory) and can be completely random. Fortunately, the size of the $\mathrm{MH}$ memory is typically much smaller (less than $128 \times 128$ ) than that of the images $(256 \times 256 \times 256)$. As a result, the internal (on-chip), high-speed memory can be used for storing the MH.

Our first strategy to meet this high memory access requirement is to use highspeed, dual-ported memory internal to the FPGA to store the MH. Because this memory is dual-ported, it can be read and written simultaneously (using different ports), an operation that is integral to $\mathrm{MH}$ accumulation. The overview of pipelined $\mathrm{MH}$ accumulation design, which takes advantage of this feature, is presented in Figure 4.11. Furthermore, because this memory can work at high speeds, the MH accumulation pipeline can work at twice the clock rate of the voxel processing 
pipeline. Thus, a pipelined implementation of MH accumulation can process (readmodify-write) two voxels per every clock of the main pipeline. Our second strategy is multiple parallel $\mathrm{MH}$ accumulation pipelines to support the high-memory access requirement. We employ four copies of the $\mathrm{MH}$ and associated parallel pipelines that independently and concurrently update the partial MHs. The partial MHs, stored in each $\mathrm{MH}$ copy, are eventually combined when the $\mathrm{MH}$ is read during the entropy calculation. Similar strategy is used for storing the individual histogram for the FI. These two strategies, when combined together, provide sufficient memory access speed to meet the requirement of $\mathrm{MH}$ accumulation.

While the $\mathrm{MH}$ is being computed, the individual histogram accumulator unit computes the histograms for the RI and the FI. These individual histograms are also stored using internal, dual-ported memories. The valid voxel counter module keeps track of the number of valid voxels accumulated in the $\mathrm{MH}$ and calculates its reciprocal value. The resulting value is then used by the entropy calculation unit for calculating the individual and joint probabilities.

\subsubsection{Data Hazards and Preaccumulation Buffers}

The presented architecture implements the operation of updating the $\mathrm{MH}$ in a pipelined fashion. This means that for each $\mathrm{MH}$ accumulation pipeline (there are four such pipelines) one interpolation weight must be accumulated per clock cycle. The MH address, which must be updated with this interpolation weight, is determined by the RI-FI intensity pair. Furthermore, the realization of this operation using dualported memories (as described above) has a latency of three clock cycles (one clock cycle each for read, modify, and write operations). As a result, read-after-write 


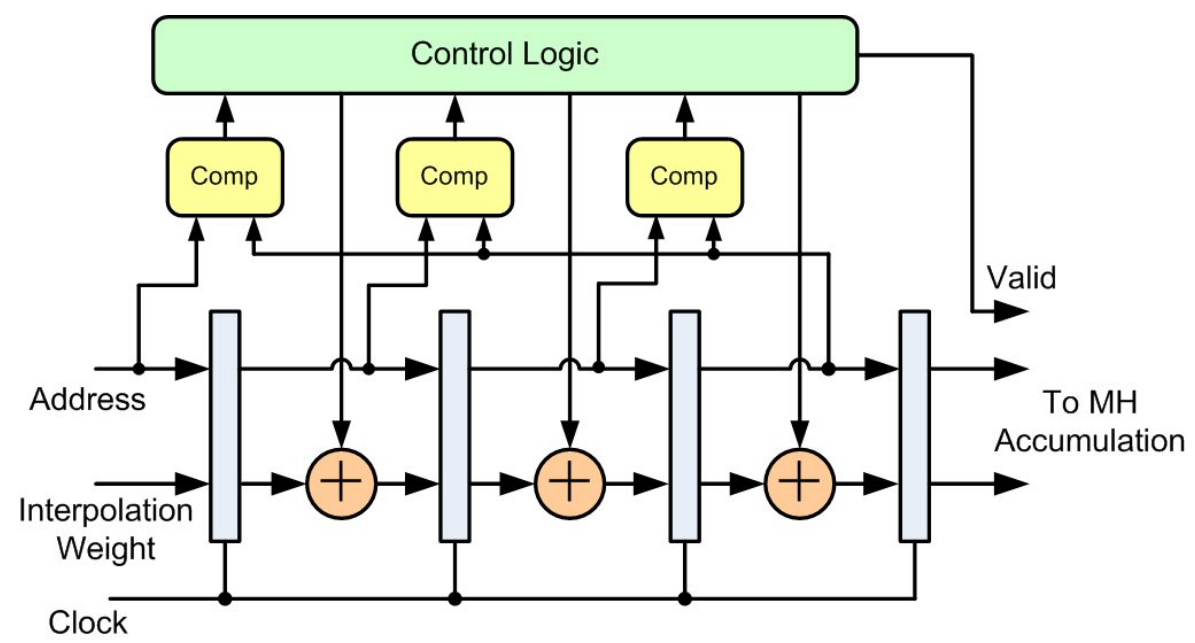

Figure 4.12: Preaccumulate buffers to eliminate RAW hazards in $\mathrm{MH}$ accumulation pipeline.

(RAW) hazards could arise if sequential $\mathrm{MH}$ update transactions that attempt to access identical locations (or in other words, transactions with same RI-FI intensity combination) are separated by fewer than three clock cycles.

To detect and eliminate these RAW hazards, the presented architecture employs a preaccumulate buffer in each pipeline. The functional block diagram of this buffer is shown in Figure 4.12. This buffer compares the address of the current transaction with that of three (equal to the latency of the operation) prior transactions. If conflicting transactions are detected, the buffer invalidates the current transaction and adds the interpolation weight of the current transaction to that of the first conflicting transaction. In summary, this is equivalent to aggregation of the weights from all the conflicting transactions and all the transactions that constitute a RAW hazard are converted into a single update to the $\mathrm{MH}$. This scheme entirely eliminates any possible RAW hazards. 


\subsubsection{Calculating $M H_{\text {Rest }}$}

During the optimization process of finding the best alignment for a given subvolume $k$ at level $i, M H_{\text {Total }_{k}}$ is computed as shown in equation (4.1). For this calculation, it is necessary to compute the contribution of the remaining subvolumes to $M H_{\text {Total }_{k}}$ using the registration information at the previous level of subdivision ( $T_{\text {parent }(j)}^{i-1}, \forall j \neq k$ ). This process must be repeated for every subvolume at the current level $i$, and the contents of $M H_{\text {Rest }_{k}}$ will be different every time depending on the subvolume under consideration. Computing $M H_{\text {Rest }_{k}}$, from scratch, for every iteration of each subvolume will not be efficient. To avoid this repeated calculation, we introduce $\mathrm{MH}$ buffers $\left(M H_{\text {Prior }}, M H_{\text {Rest }}\right.$, and $\left.M H_{\text {Local }}\right)$, which store the previous-level $\mathrm{MH}$ and partial $\mathrm{MH}$ during various stages of the algorithm. A flow diagram depicting the interplay between these MH buffers during various steps of calculating $M H_{\text {Rest }}$ is shown in Figure 4.13, and the detailed description is provided here.

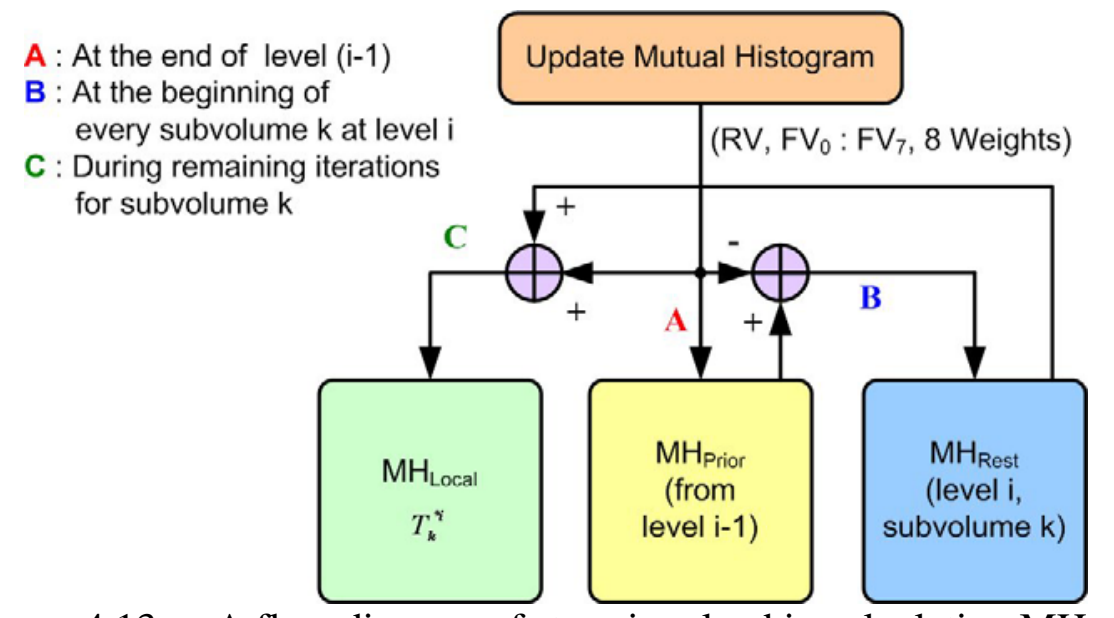

Figure 4.13: A flow diagram of steps involved in calculating $\mathrm{MH}_{\text {Rest }}$. 
At a given level $i, M H_{\text {Prior }}$ contains the $\mathrm{MH}$ for the entire image, which is computed using all the subvolumes at the earlier level $i-1$ and corresponding transformations $T_{j}^{i-1}$. At the beginning of the registration of each subvolume $k$ at the current level $i, M H_{\text {Local }}$ is cleared (thus, all its entries are set to 0). Next, the transformation of its parent from the previous level, $T_{\text {parent }(k)}^{i-1}$, is applied to the current subvolume $k$ and the resultant $\mathrm{MH}$ is accumulated in $M H_{\text {Local }}$. $M H_{\text {Local }}$ now contains the contribution of the subvolume $k$ to $M H_{\text {Prior }}$. $M H_{\text {Local }}$ is then subtracted from $M H_{\text {Prior }}$, and the resultant $\mathrm{MH}$ is stored in the buffer $M H_{\text {Rest }}$. This step is mathematically equivalent to computing $M H_{\text {Restk }_{k}}$ (as in equation (4.3)) for the subvolume $k$. For every subsequent optimization iteration involving this subvolume, $M H_{\text {Local }}$ is initially cleared. The candidate transformation $T_{k}^{*_{i}}$ is then applied to the current subvolume $(k)$ only, and the resultant histogram (contribution of this subvolume) is accumulated in $M H_{\text {Local }}$. It is then added to $M H_{\text {Restk }}$, to form the $\mathrm{MH}$ for the entire image ( $M H_{\text {Total }_{k}}$ in equation (4.1)) for the current optimization step. This final histogram is then further used for computing the image similarity measure (MI) corresponding to the current transformation $T_{k}^{*_{i}}$. This process is repeated for all the subvolumes at the current level. At the end of each level (after optimizing all the subvolumes at that level), the $\mathrm{MH}$ for the entire image is computed using the updated transformations $T_{k}^{i}, \forall k$ and is stored in $M H_{\text {Prior }}$, which will then subsequently be used at the next level of subdivision, $i+1$. 


\subsubsection{Entropy Calculation}

Acceleration of $\mathrm{MH}$ accumulation contributes to the most of the performance improvement during calculation of MI. However, the final step in MI calculation, which is entropy calculation, must also be implemented in the hardware to eliminate the overhead of copy the MH back to the host. The mutual and individual entropies are computed by using the probability distributions represented by the mutual and individual histograms, respectively.

To calculate entropy, it is necessary to evaluate the function $f(p)=p \cdot \ln (p)$ for all the probabilities. As probability $p$ takes values between $[0,1]$, the corresponding range for the function $\ln (p)$ is $[-\infty, 0]$. In addition, $\ln (p)$ is undefined for $p=0$. In comparison, the corresponding range for $f(p)$ is $\left[-e^{-1}, 0\right]$. Thus, $f(p)$ has a finite dynamic range and is defined for all values of $p$. Several methods for calculating logarithmic functions in hardware have been reported earlier [152-154], but of particular interest is the multiple lookup table (LUT)-based approach introduced by Castro-Pareja et al. [155]. This approach minimizes the error in representing $f(p)$ for a given number and size of LUTs and, hence, is accurate and efficient. Moreover, this approach preserves the shape of the MI curve and the location of the extrema and thus does not significantly affect the outcome of the optimization process. Following this approach, the presented design implements $f(p)$ using multiple LUT-based piecewise polynomial approximation. We provide a brief overview of this method here and identify the multiple LUT configuration adopted by our architecture. 


\subsubsection{Multiple LUT-Based Approach for Entropy Calculation}

A LUT-based approach represents the target function (such as $f(p)$ ) using a piecewise-polynomial approximation with $n$ segments. In particular, approximation using Chebyshev polynomials is employed because: 1) the Chebyshev approximation is simple to calculate for continuous functions such as $f(p)$ and 2) it is very close to the true minimax approximation, the most accurate polynomial approximation. The polynomials used can be of arbitrarily high order. Higher degree of polynomials typically offer better approximation, but require higher degree of arithmetic computation (and associated hardware resources) for function evaluation. For this reason, only $1^{\text {st }}$ order polynomials are considered.

In case of a single-LUT based approach, all the segments are stored in a single large LUT. Although this approach is simple and allows easy decoding and addressing during function evaluation, it suffers from several drawbacks. First, the precision of the LUT $(\Delta p)$ is constant throughout the entire table. As a result, segments of the approximated function that require finer precision can not be accurately represented. Second, the size of the LUT to achieve a desired approximation error is usually quite large and sometimes not practical. For example,

Table 4.1: Configurations of LUT-based entropy calculation module that were considered in the presented architecture.

\begin{tabular}{ccccccc}
\hline \hline \multirow{2}{*}{ LUT No. } & \multicolumn{2}{c}{ LUT Range } & \multicolumn{2}{c}{ Configuration \#1 } & \multicolumn{2}{c}{ Configuration \#2 } \\
\cline { 2 - 7 } & $p_{\min }$ & $p_{\max }$ & $\Delta p$ & Entries & $\Delta p$ & Entries \\
\hline 1 & 0 & $2^{-13}$ & $2^{-23}$ & 1024 & $2^{-24}$ & 2048 \\
2 & $2^{-13}$ & $2^{-6}$ & $2^{-16}$ & 1024 & $2^{-17}$ & 2048 \\
3 & $2^{-6}$ & $2^{-2}$ & $2^{-12}$ & 1024 & $2^{-13}$ & 2048 \\
4 & $2^{-2}$ & 1 & $2^{-10}$ & 1024 & $2^{-11}$ & 2048 \\
\hline \hline
\end{tabular}


to keep the error in entropy calculation less than $10^{-6}$, a single LUT with $16 \mathrm{~K}$ entries will be required.

The multiple LUT-based approach introduced by Castro-Pareja et al. [155] attempts to address these limitations. In this approach, each segment is stored in a different LUT. In addition, each LUT has a different precision ( $\Delta p)$ and uses a different polynomial coefficients to represent the individual segments of the function $f(p)$. Thus, this approach allows superior approximation of the target function and thereby keeping the approximation error below the allowable maximum. Furthermore, this method provides a realization that is optimized for approximation accuracy and hardware resources [155].

Using this approach the $1^{\text {st }}$-order polynomial, 4-LUT configuration was designed. We realized two such configurations each with a different LUT precision $(\Delta p)$. These configurations are listed in Table 4.1. The choice of these configurations

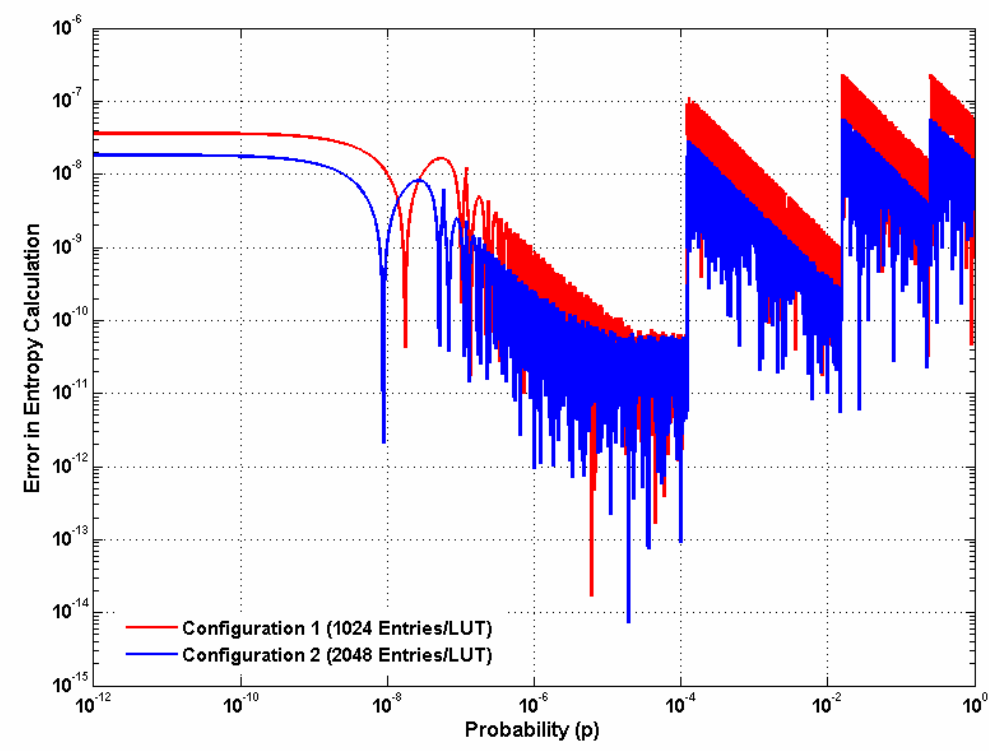

Figure 4.14: Error in entropy calculation corresponding to the two configurations of the multiple LUT-based implementation. 
was based on meeting the error constraints on the entropy calculation and the availability of resources in the target FPGA device. We then evaluated the approximation error offered by these two realizations. This was done by comparing the entropy values calculated by using these designs against that calculated using a double-precision software implementation. Figure 4.14 shows a plot of the error magnitude vs. $p$ for both these realizations. The maximum error was of the order of $10^{-8}$ for a both these configurations, which is sufficient for MI calculation in the context of image registration [155]. For our architecture we selected realization with $8 \mathrm{~K}$ entries, as it offers superior error performance at a relatively moderate increase in the LUT size. As described earlier, using the lower-order polynomials has the advantage of reducing the LUT data width and the number of required arithmetic operations for function evaluation.

\subsubsection{Operational Workflow}

The sections above described the design and functional behavior of the important modules in the architecture we developed for accelerated calculation of MI. Our ultimate goal, however, is to accelerate deformable image registration algorithm described earlier. This algorithm utilizes this high-speed implementation to efficiently calculate the image similarity measure (MI), the most computationally complex step within the algorithm. This deformable registration algorithm is based on volume subdivision, and depending on the operational stage of the algorithm, image similarity is calculated in a slightly different manner. For example, steps to calculate MI during rigid registration are slightly different than those taken during optimization of a subvolume after volume subdivision. This section identifies different operational 
stages of the algorithm and describes how the architecture described above is utilized to calculate MI during those stages. The summary of this description is provided in Table 4.2.

\subsubsection{Rigid Registration}

Rigid registration is the first step in the aforementioned deformable registration algorithm. This step attempts to recover any gross misalignment between the RI and FI. During this step, the algorithm repeatedly calculates MI for various candidate transformations between RI and FI. To implement this operation using the described architecture, we first clear all the mutual histograms internal to the architecture $\left(M H_{\text {Prior }}, M H_{\text {Rest }}\right.$, and $\left.M H_{\text {Local }}\right)$. Next, the host provides the current candidate transformation along with the subvolume address range (equal to the RI dimensions, since MI is calculated for the entire image) to the architecture. After this operation, the $\mathrm{MH}$ corresponding to the given transformation is calculated and stored

Table 4.2: Operational workflow for performing volume subdivision-based deformable image registration using the presented architecture.

\begin{tabular}{cc}
\hline \multicolumn{1}{c}{ Operation } & Workflow \\
\hline Rigid Registration & Clear All MHs $\rightarrow$ Accumulate (entire RI) $\rightarrow$ Calculate \\
Entropy using $\mathrm{MH}_{\text {Local }}$
\end{tabular}


in $M H_{\text {Local }}$. Later on, the individual and joint entropies are calculated using $M H_{\text {Local }}$.

\subsubsection{Calculating $M H_{\text {Prior }}$}

Calculating $M H_{\text {Prior }}$ requires applying transformations to all the subvolumes at a given level of subdivision and calculating the cumulative $\mathrm{MH}$ corresponding to all subvolumes with their associated transformations. To implement this operation using the described architecture, we first clear $M H_{\text {Local }}$, which is used for subvolume $\mathrm{MH}$ accumulation. Next, the host provides the address range for the first subvolume and the transformation associated with that subvolume from the previous level. The MH corresponding to the given transformation is calculated and stored in $M H_{\text {Local }}$. This step is repeated for all the subvolumes at a given level of subdivision, with a difference that $M H_{\text {Local }}$ is cleared only during the first subvolume. This allows the calculation of the cumulative $\mathrm{MH}$ for all the subvolumes. After all the subvolumes are processed, $M H_{\text {Local }}$ is copied to $M H_{\text {Prior }}$ (an internal $\mathrm{MH}$ buffer). No entropy calculation is required during this step. This step is repeated after each level of image subdivision.

\subsubsection{Calculating $M H_{\text {Rest }}$}

Calculation of $M H_{\text {Rest }}$ is a prerequisite for optimizing subvolumes after volume subdivision. As described earlier, we utilize $M H_{\text {Prior }}$ to efficiently calculate $M H_{\text {Rest }}$ for a given subvolume. To implement this operation using our architecture, we first clear $M H_{\text {Local }}$, which is used for subvolume MH accumulation. Next, the host 
provides the address range for the current subvolume and the transformation associated with that subvolume from the previous level. The MH corresponding to the given transformation is calculated and stored in $M H_{\text {Local }}$. Following this operation, we subtract $M H_{\text {Local }}$ from $M H_{\text {Prior }}$ and store the resulting $\mathrm{MH}$ into $M H_{\text {Rest }}$ (another internal MH buffer). This step is repeated prior to registration of every subvolume at a given level of subdivision.

\subsubsection{Subvolume Registration}

After image subdivision, the resulting subvolumes are individually registered with the FI. However, the image registration algorithm also considers the contribution of the rest of the image for improved statistical reliability. Our architecture supports this operation by taking advantage of the $M H_{\text {Rest }}$ computed in the previous step. First, we clear $M H_{\text {Local }}$, which is used for subvolume $\mathrm{MH}$ accumulation. Next, the host provides the address range for the current subvolume and the current candidate transformation for that subvolume. The $\mathrm{MH}$ corresponding to the given candidate transformation is calculated and stored in $M H_{\text {Local }}$. Later on, the individual and joint entropies are calculated using $\left(M H_{\text {Local }}+M H_{\text {Rest }}\right)$, thus taking into account the contribution of the rest of the image. This step is repeated for each iteration during the registration of a subvolume.

\subsection{Implementation and Results}

The architecture presented above was implemented using an Altera Stratix II EP2S180F1508C4 FPGA (Altera Corp., San Jose, CA) in a PCI prototyping board 
(DN7000K10PCI) manufactured by the Dini Group (La Jolla, CA). The board featured 1 GB double-data-rate (DDR2) DRAM in a small-outline dual-inline memory module (SoDIMM) external to the FPGA. This memory was used to store the RI and the FI. The board provided a 64-bit bus interface between the memory and the FPGA running at $200 \mathrm{MHz}$ clock speed. The architecture was designed using VHSIC hardware description language (VHDL) and synthesized using Altera Quartus II 6.1. The memory controller was also implemented using VHDL and was built around the DDR2 DRAM controller megacore supplied with Altera Quartus II. All the modules in the architecture were custom designed using VHDL, per the design description provided earlier. Functional verification and postsynthesis timing simulation for the entire system were performed using Modelsim SE 6.2 (Mentor Graphics, San Jose, CA). For this purpose, DDR2 DRAM simulation models provided by Micron (www.micron.com) were used. The resource availability of the target FPGA, primarily internal memory, which is used for MH accumulation, limited the synthesis of the reported design to support 7-bit mutual histogram and, consequently, all results in this section are presented for this configuration. In comparison, the software implementation uses 8-bit mutual histogram. In spite of this difference, we demonstrate that the registration accuracy for both these implementations is comparable (see Chapter 6), which further confirms the findings of Studholme et al. [64].

To verify the functional correctness and evaluate the performance of this implementation we considered image registration between intraprocedural noncontrast CT (iCT) with preprocedural contrast-enhanced CT (preCT) and positron 
emission tomography (PET) images. The registration between these image-pair combinations is clinically relevant and routinely encountered in the context of CTguided interventions. We considered five abdominal iCT-preCT and five abdominal iCT-PET image pairs for this evaluation. The image size for iCT and preCT was 256 $\times 256 \times 200-350$ with a voxel size of $1.4-1.7 \mathrm{~mm} \times 1.4-1.7 \mathrm{~mm} \times 1.5 \mathrm{~mm}$, whereas the typical image size for PET was $128 \times 128 \times 154-202$ voxels with a voxel size of $5.15 \mathrm{~mm} \times 5.15 \mathrm{~mm} \times 5.15 \mathrm{~mm}$. The iCT, preCT, and PET images were converted to 8 bits and 7 bits, respectively, for software and FPGA-based implementations. This conversion was performed using adaptive reduction in intensity levels [156]. The converted iCT and preCT images were then preprocessed using 3D anisotropic diffusion filtering. No preprocessing steps were used for the PET images. The realtime implementation of anisotropic diffusion filtering that we presented earlier can facilitate this preprocessing without adding any significant latency to IGI workflow [144]. For the hardware implementation, the RI was stored in a sequential format organized by subvolumes, while eight interleaving copies of the FI were arranged in memory to facilitate cubic addressing. This arrangement allows simultaneous access to an entire 3D neighborhood within the FI, as described earlier. The execution speed of the reported architecture was obtained from postsynthesis timing measurements using the entire system.

The design achieved a maximum internal frequency of $200 \mathrm{MHz}$, with a theoretical maximum RI voxel processing rate of $100 \mathrm{MHz}$. The coordinate transformation, PV interpolation, and $\mathrm{MH}$ accumulation operations were implemented using fixed-point representation. Entropy calculation was implemented 
using the 4-LUT, first-order polynomial configuration as described earlier. The precision employed for this fixed-point datapath can affect both the implementation accuracy and the hardware resource requirement of this architecture. An optimization framework to systematically explore this effect is presented in the following chapter. This framework is capable of identifying the optimized tradeoff relationship between the hardware resources and the accuracy. The optimization of our architecture by using this framework is presented in Chapter 5. We further evaluated the accuracy of deformable image registration offered by that optimized architectural configuration and those results are presented in Chapter 6. In this section, however, we focus on the execution performance of our architecture and demonstrate its functional correctness through qualitative validation of image registration. For this purpose we used a designer identified architectural configuration which was not optimized for areaimplementation error tradeoff. Consequently, all the results presented in this section are corresponding to that design configuration.

\subsubsection{Execution Speed}

The presented architecture is targeted toward accelerating the calculation of MI for a hierarchical volume subdivision-based deformable registration algorithm. During the execution of this algorithm, MI must be repeatedly calculated under a candidate transformation for every subvolume at every level of subdivision. Moreover, as described earlier, $M H_{\text {Rest }}$ must be calculated once for every subvolume. To analyze the speedup offered by the presented FPGA-based solution for calculation of MI, we compare its calculation time with that of a software $(\mathrm{C}++)$ implementation running on an Intel Xeon 3.6 GHz workstation with 2 GB of RAM. Table 4.3 details 
Table 4.3: Comparison of mutual information calculation time for subvolumes at various levels in volume subdivision-based deformable registration algorithm.

\begin{tabular}{|c|c|c|c|c|}
\hline \multirow{2}{*}{$\begin{array}{l}\text { Subdivision } \\
\text { level }\end{array}$} & \multirow{2}{*}{$\begin{array}{l}\text { Subvolume } \\
\text { size }\end{array}$} & \multicolumn{2}{|c|}{$\begin{array}{c}\text { Mutual information } \\
\text { calculation time (ms) }\end{array}$} & \multirow{2}{*}{ Speedup } \\
\hline & & $\begin{array}{c}\text { Software } \\
\text { implementation }\end{array}$ & $\begin{array}{c}\text { FPGA-based } \\
\text { implementation }\end{array}$ & \\
\hline $0^{\mathrm{a}}$ & $256 \times 256 \times 256$ & 9410 & 225.42 & 41.74 \\
\hline 1 & $128 \times 128 \times 128$ & 1209 & 30.19 & 40.05 \\
\hline 2 & $64 \times 64 \times 64$ & 166 & 4.16 & 39.90 \\
\hline 3 & $32 \times 32 \times 32$ & 18 & 0.78 & 23.08 \\
\hline 4 & $16 \times 16 \times 16$ & 10 & 0.46 & 21.74 \\
\hline
\end{tabular}

${ }^{\mathrm{a}}$ This corresponds to rigid registration between the reference and floating images.

this performance comparison for a iCT-preCT image pair with dimensions of $256 \times$ $256 \times 256$. The last column of the table shows the speedup offered by the reported solution over the software implementation for a given level of subdivision. The time to calculate MI primarily depends on the size of the subvolume and is independent of the imaging modality and voxel size. This calculation time, however, may vary slightly based on the actual value of the transformation used to calculate MI. These variations are caused by differences in access patterns to the FI memory under different transformation values. To compensate for this effect and report average MI calculation time at a given level of subdivision, we measured the MI calculation times for a subvolume using 100 randomly generated transformations (within the range of \pm 30 voxels for translations and $\pm 20^{\circ}$ for rotations).

The same set of transformations was used for both software and hardware implementations. The MI calculation time reported in Table 4.3 is averaged over all the transformations. Hardware timings reported in Table 4.3 also include the communication time, required for writing the transformation matrix and reading back 
the calculated entropy values, between the host and the MI calculator. Furthermore, consistent with the scenario during the execution of the registration algorithm, the time to compute $M H_{\text {Rest }}$ (once per subvolume) is also included in the hardware and software MI calculation time. Our acrchitecture offers a speedup of about 40 for calculating MI up to subvolumes of size $64^{3}$; whereas for smaller subvolumes the speedup achieved is around 20. This drop in achieved speedup is explained by taking into account overheads incurred during computation of MI. Calculating MI requires accumulation of $\mathrm{MH}$, which in turn, requires initial clearing of the MH memory. Because the current implementation employs an MH with size $128 \times 128$ (to support 7-bit images), the process of clearing $\mathrm{MH}$, which involves writing '0's to all $\mathrm{MH}$ entries, can consume more than 16,000 clock cycles. For smaller subvolumes, the time required to clear $\mathrm{MH}$ memory becomes comparable to or larger than that required to process a subvolume (images are processed at the rate of approximately one voxel per clock cycle). In addition, the communication time between the host and the MI calculator, required for exchanging the transformation matrix and the calculated MI value, becomes comparable to the computation time. These two factors limit the net speedup achieved for smaller subvolumes.

Table 4.4 compares the total execution time for deformable registration using a software $(\mathrm{C}++)$ implementation running on an Intel Xeon $3.6 \mathrm{GHz}$ workstation with

Table 4.4: Execution time of deformable image registration.

\begin{tabular}{cccc}
\hline \hline Image pair & \multicolumn{2}{c}{ Execution time (s) } & \\
\cline { 2 - 3 } $\begin{array}{c}\text { used for } \\
\text { registration }\end{array}$ & $\begin{array}{c}\text { Software } \\
\text { implementation }\end{array}$ & $\begin{array}{c}\text { FPGA-based } \\
\text { implementation }\end{array}$ & \\
\hline iCT-preCT & 11520 & 371 & 31.05 \\
iCT-PET & 11146 & 339 & 32.88 \\
\hline \hline
\end{tabular}


2 GB of RAM against the presented FPGA-based architecutre. This execution time was measured for deformable registration between five iCT-preCT and five iCT-PET image pairs, described earlier. The maximum number of global and local iterations was set to 200 and 100 respectively. The same optimization algorithm was used for both the software and hardware implementations, and volume subdivision continued until the subvolume size was larger than $16^{3}$. For each image modality pair, the execution time reported is the average of execution times of the five cases. The execution time of intensity-based image registration is directly proportional to the size of the RI. iCT image was used as the RI for both the image modality pairs and, hence, the execution time for deformable registration is similar for these two image modalities, despite the fact that PET images are smaller than preCT images $(128 \times$ $128 \times 154-202$ and $256 \times 256 \times 200-350$, respectively). For both image modality pairs, our architecture provided a speedup of about 30 over an equivalent software implementation and achieved an execution time of around 6 minutes. This speedup is a direct outcome of acceleration of MI calculation using the presented architecture.

\subsubsection{Performance Comparison}

Intensity-based image registration has been identified to be a computationally intensive problem. Given the impact the acceleration of image registration can have on a variety of diagnostic and interventional applications, there have been significant efforts towards realizing high-speed implementations of image registration algorithms. Further, given that this problem is extremely compute-extensive and multi-faceted; researchers have applied a range of computing platforms and acceleration strategies at different levels of this problem to improve its execution 
performance. In Chapter 2, we provided a survey of some acceleration approaches that are somewhat related to the work presented in this dissertation. In this section we compare the performance improvement provided by the FPGA-based implementation we developed in this dissertation work against that provided by the earlier reported solutions.

For this comparison we focused on acceleration approaches involving intensity-based image registration, since these approaches can be retrospective, automatic and have the potential to be integrated into the clinical workflow. The algorithms considered included FFD-based, and gradient flow-based approaches as well as those based on the demon's algorithm. Moreover, these implementations used different similarity measures (MI, NMI, SSD etc.), transformation models (splinebased, rigid, volume-subdivision based) and acceleration strategies as dictated by the computational platform (GPUs, clusters, FPGAs etc.) used for their implementation. Thus, there is a substantial variation in the implementation details (both, for original and accelerated implementation) of each of these realizations, even though they try to address the same fundamental problem of accelerating intensity-based registration. To account for these variations and provide a fair comparison, we consider the net speedup offered by these accelerated implementations against their corresponding unaccelerated versions. This comparison can be used to judge the efficiency of various strategies to accelerate intensity-based image registration. To compare the raw computational performance these realizations, we normalize their corresponding execution times by the dimensions of reference image used for registration and calculate the effective voxel processing rate (throughput) offered. In some cases, 
where sufficient implementation details (such as number of iterations, number of voxels processed etc.) were not provided the voxel processing rate could not be calculated.

Table 4.5 presents the results of this analysis and compares the performance of the presented architecture for high-speed implementation of deformable image registration, against a corresponding software implementation and previously reported acceleration approaches. The software implementation refers to the implementation of the volume subdivision-based algorithm. This implementation was developed using $\mathrm{C}++$, and its performance was measured on an Intel Xeon $3.6 \mathrm{GHz}$ workstation with 2 gigabytes of DDR2 $400 \mathrm{MHz}$ main memory. The presented implementation of FPGA-based MI calculation provides more than an order of magnitude speedup and superior voxel processing rate when compared to this software implementation.

The majority of earlier reported attempts to accelerate intensity-based image registration have primarily employed a multiprocessor or supercomputer approach (see [57, 116, 117], for example). Although these solutions delivered high performance and speedup by virtue of parallelization and high-speed network interconnects, the speedup achieved per processor was less than unity. Also, in some cases [116] the voxel processing rate achieved was substantially less than that offered by the presented implementation. In addition, these solutions may not be cost effective and, because of their size, are unlikely to be suitable for clinical deployment. Some solutions based on graphics processors [120, 122] attempt to provide performance improvement by exploiting data parallelism native to this platform. 
Although this platform yielded reasonable speedups for rigid registration, the performance gain and voxel throughput for deformable registration and in particular, for that based on MI, were poor. Ohara et al. [123] attempted to accelerate MI-based rigid registration using a pair of cell processors, by exploiting the inherent parallelism offered by multiple cores (8 per processor) and high memory bandwidth. Using these 16 computing cores, this implementation achieved speedup of only 11 for rigid registration. The voxel throughput of this implementation (6 MHz), however, was slightly better when compared with other multiprocessor implementations. This can be attributed to the high memory bandwidth and additional designer optimizations to take advantage of spatial locality. Jiang et al. [124] employed a high-level description language (Handel-C) and mapped B-spline computation (a common component in FFD-based image registration) to Xilinx FPGA devices. This implementation leveraged elimination of nested loops and pipelined implementation to achieve performance improvement. Despite the semi-automated nature of this approach and high clock rate of the synthesized design, the speedup and the voxel throughput achieved were limited to 3.2 and $4 \mathrm{MHz}$ respectively.

In comparison with these acceleration approaches, the FPGA-based implementation developed in this dissertation addresses the fundamental memory access bottleneck in intensity-based image registration. Furthermore, voxel-level parallelism is achieved through pipelined implementation and this implementation can offer voxel processing rate close to one voxel per clock cycle. Thus, this solution is not only compact, but offers high speedup (around 30) and superior voxel throughput (> $70 \mathrm{MHz}$ ) using a single processing element. 
Table 4.5: Performance comparison of the presented FPGA-based implementation of intensity-based deformable image registration with an equivalent software implementation and prior approaches for acceleration of intensity-based registration.

\begin{tabular}{|c|c|c|c|c|}
\hline Implementation & Algorithm & Platform & Speedup & $\begin{array}{c}\text { Voxel } \\
\text { processing } \\
\text { rate (MHz) } \\
\text { (if available) }\end{array}$ \\
\hline $\begin{array}{l}\text { Stefanescu } \\
\text { et al. [116] }\end{array}$ & $\begin{array}{l}\text { Demon's } \\
\text { algorithm }\end{array}$ & $\begin{array}{l}\text { 15-processor } \\
\text { cluster }\end{array}$ & 11 & 1.8 \\
\hline $\begin{array}{l}\text { Rohlfing } \\
\text { et al. [57] }\end{array}$ & $\begin{array}{l}\text { FFD-based } \\
\text { deformable, } \\
\text { using MI }\end{array}$ & $\begin{array}{l}\text { 64-processor } \\
\text { shared-memory } \\
\text { supercomputer }\end{array}$ & 40 & - \\
\hline $\begin{array}{c}\text { Ino } \\
\text { et al. [117] }\end{array}$ & $\begin{array}{c}\text { FFD-based } \\
\text { deformable, using } \\
\text { MI }\end{array}$ & $\begin{array}{c}\text { 128-processor } \\
\text { cluster, Myrinet } \\
\text { connectivity }\end{array}$ & 90 & - \\
\hline $\begin{array}{c}\text { Kohn } \\
\text { et al. [120] }\end{array}$ & $\begin{array}{l}\text { Gradient flow, } \\
\text { rigid/deformable }\end{array}$ & $\begin{array}{l}\text { GPU (6800) } \\
\text { (using GLSL) }\end{array}$ & 12 & 2.15 \\
\hline $\begin{array}{c}\text { Vetter } \\
\text { et al. [122] }\end{array}$ & $\begin{array}{l}\text { MI-based } \\
\text { deformable }\end{array}$ & $\begin{array}{l}\text { GPU (7800) } \\
\text { (using GLSL) }\end{array}$ & 6 & 3.21 \\
\hline $\begin{array}{c}\text { Ohara } \\
\text { et al. [123] }\end{array}$ & $\begin{array}{l}\text { MI-based } \\
\text { rigid }\end{array}$ & $\begin{array}{l}2 \text { cell broadband } \\
\text { engine processors }\end{array}$ & 11 & 5.75 \\
\hline $\begin{array}{c}\text { Jiang } \\
\text { et al. [124] }\end{array}$ & $\begin{array}{c}\text { B-spline } \\
\text { interpolation }\end{array}$ & $\begin{array}{c}\text { FPGA } \\
\text { (using Handel-C) }\end{array}$ & 3 & 4.19 \\
\hline $\begin{array}{l}\text { Software } \\
(\mathrm{C}++)\end{array}$ & $\begin{array}{c}\text { MI-based } \\
\text { deformable }\end{array}$ & Xeon workstation & - & 1.75 \\
\hline $\begin{array}{l}\text { Dandekar } \\
\text { et al. [157] }\end{array}$ & $\begin{array}{c}\text { MI-based } \\
\text { deformable }\end{array}$ & FPGA & 33 & 74.43 \\
\hline
\end{tabular}




\subsubsection{Qualitative Evaluation of Deformable Image Registration}

Acceleration of deformable registration, as offered by the presented architecture, is critical for the time-sensitive nature of IGIs; however the accuracy of the registration process is of equal importance. As described earlier, we present the results of qualitative validation in this section, which demonstrate the functional correctness of our architecture. The results of the quantitative validation are presented later, in Chapter 6. The qualitative validation of deformable registration was performed using five iCT-preCT and five iCT-PET image pairs. For all the cases visually correct alignment was achieved after deformable image registration. Furthermore, the results of the registration performed using our architecture was qualitatively similar to that obtained using the software implementation.

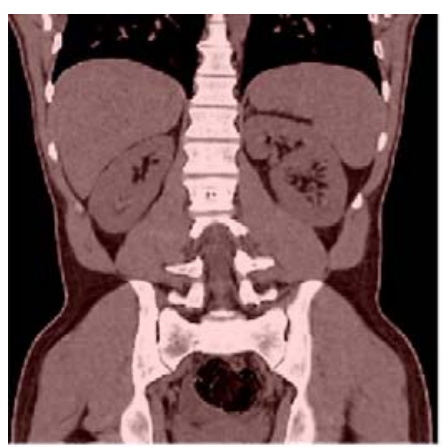

(a)

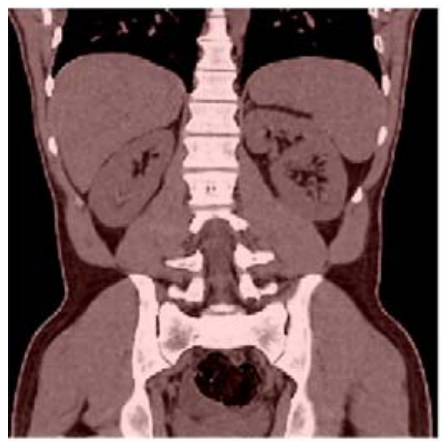

(d)

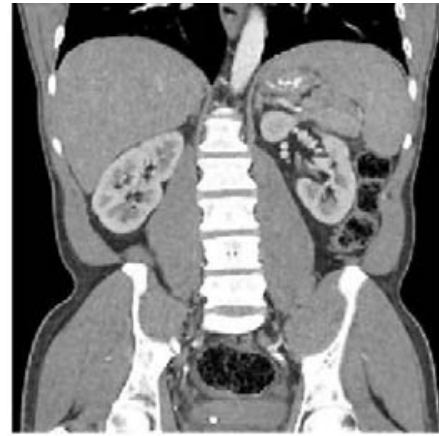

(b)

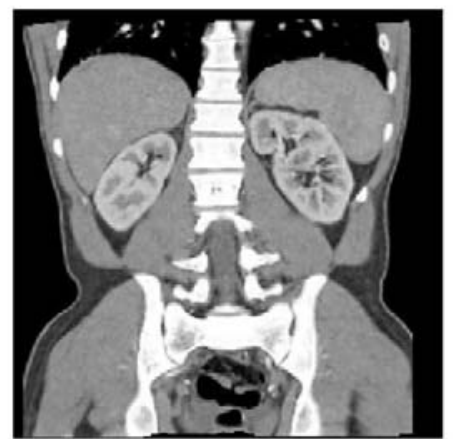

(e)

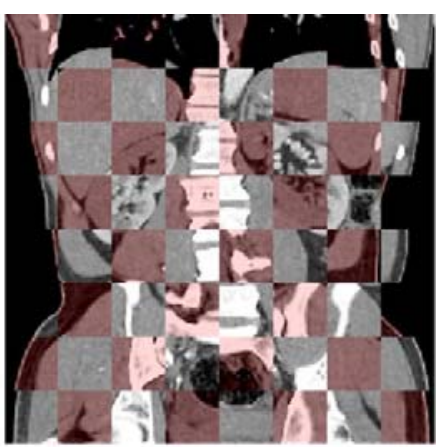

(c)

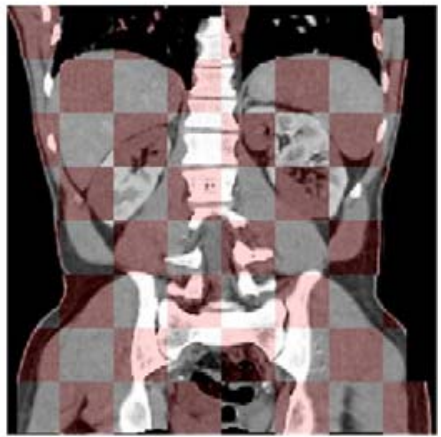

(f)

Figure 4.15: Qualitative validation of deformable registration between iCT and preCT images performed using the presented FPGA-based solution. 
Figure 4.15 shows an example of deformable registration between a representative pair of iCT and preCT images. This registration was performed using the presented FPGA-based solution. The initial seed alignment between these images was obtained using a single-click operation to compensate for different scanner coordinate systems and ensure reasonable overlap of common regions between two images. Subfigures (a) and (b) show coronal slices from iCT and preCT images, respectively. Subfigure (c) shows the overlay of these two images using the checkerboard pattern. In this checkerboard overlay, blocks from both the images are displayed alternately. The structural misalignment between iCT and preCT images is evident from mismatches at the boundaries of these blocks. Subfigure (e) shows a coronal slice from the preCT image registered to the iCT image, and subfigure (f) shows the overlay of this image with the original iCT image. Better structural alignment after deformable registration is evident from improved matching at the boundaries of the blocks of the checkerboard overlay.

Similarly, Figure 4.16 shows an example of deformable registration between a representative pair of iCT and PET images. This registration was also performed using the reported FPGA-based solution. The initial alignment between these two

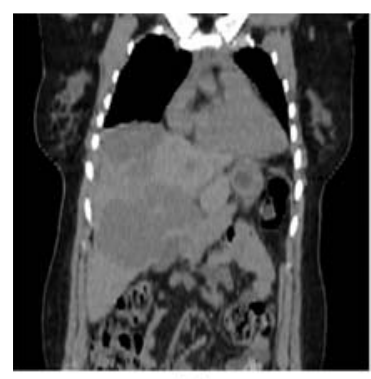

(a)

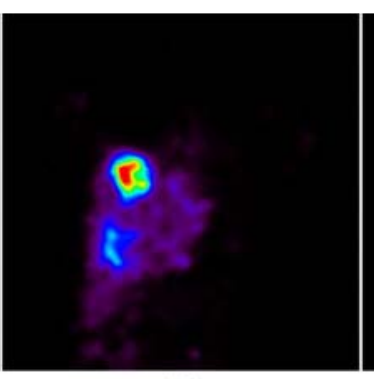

(b)

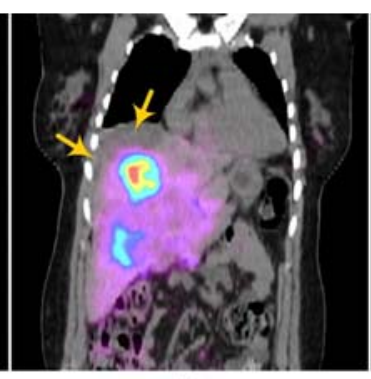

(c)

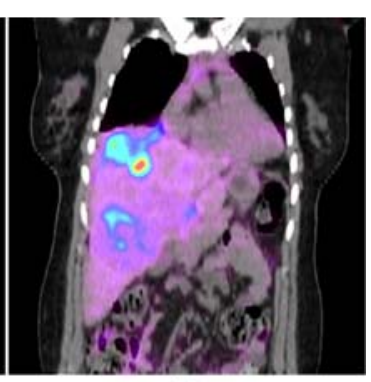

(d)

Figure 4.16: Qualitative validation of deformable registration between iCT and PET images performed using the presented FPGA-based solution. 
images was obtained as for the previous case. Subfigures (a) and (b) show coronal slices from iCT and PET images, respectively. Subfigure (c) shows the fusion of these two images. Subfigure (d) shows the fusion of the registered PET image with the original iCT image. Improved alignment after deformable registration is evident from better matching of structures in the fusion image.

\subsection{Summary}

In this chapter we presented a novel FPGA-based architecture for high-speed implementation of MI-based deformable image registration. This architecture achieved voxel-level parallelism through pipelined implementation and employed several strategies to address the fundamental bottleneck in the intensity-based image registration, namely memory access management. As a result of these enhancements, the presented architecture is capable of achieving high voxel processing rate and a speedup of about 30 and consequently reduces the execution time of deformable registration from hours to only a few minutes. The results of the qualitative validation indicate that this performance improvement does not significantly compromise the accuracy of deformable registration. As qualitatively presented in this chapter and demonstrated quantitatively later (see Chapter 6), this implementation enables improved target delineation during image-guided interventions through deformable registration with preprocedural images. The robustness, speed, and accuracy offered by this architecture, in conjunction with its compact implementation, make it ideally suitable for integration into IGI workflow.

Accurate, robust, and real-time deformable image registration between intraand preprocedural images is an unmet need, critical to the success of image-guided 
procedures. The work presented in this chapter of the dissertation represents an important first step toward meeting this goal. With further algorithmic and hardware improvements, geared toward enhancing its accuracy and performance, this approach has the potential to elevate the precision of current procedures and expand the scope of IGI to moving and deformable organs. 


\section{Chapter 5: Framework for Optimization of Finite Precision}

\section{Implementations}

Computationally intensive algorithmic components are routinely accelerated by mapping them to custom or reconfigurable hardware platforms. The work presented in the earlier chapters of this dissertation has a similar flavor. An important consideration for these implementations, which often employ finite precision datapaths, is the tradeoff between hardware resources and implementation accuracy. This chapter presents a methodology for systematically exploring this tradeoff and identifying design configurations that efficiently balance these two conflicting objectives. First, we formulate this problem as a multiobjective optimization problem. Next, we present a multiobjective optimization framework developed in the context of FPGA-based architecture for image registration presented in the previous chapter. Finally, we demonstrate the applicability of the proposed approach through simulation and post-synthesis validation results.

\subsection{Motivation}

An emerging trend in real-time signal processing systems is to accelerate computationally intensive algorithmic components (for example, MI calculation as described in the previous chapter) by mapping them to custom or reconfigurable hardware platforms, such as ASICs and FPGAs. Most of these algorithms are initially developed in software using floating-point representation and later migrated to hardware using finite precision (e.g., fixed-point representation) for achieving improved computational performance and reduced hardware cost. These 
implementations are often parameterized, so that a wide range of finite precision representations can be supported [158] by choosing an appropriate wordlength for each internal variable. As a consequence, the accuracy and hardware resource requirements of such a system are functions of the wordlengths used to represent the internal variables. Determining an optimal wordlength configuration has been shown to be NP-hard [159] and can take up to 50\% of the design time for complex systems [125]. Moreover, a single optimal solution may not exist, especially in the presence of multiple conflicting objectives, as is the case in the current work. In addition, a new configuration generally needs to be derived when the design constraints or application requirements are altered.

An exhaustive search of the entire design space is guaranteed to find Paretooptimal configurations. Execution time for such exhaustive search, however, increases exponentially with the number of design parameters, making it unfeasible for most practical systems. Some earlier reported techniques have attempted to solve this problem through the use of analytical modeling [86-90], gradient-based search [93], linear programming strategies [86], or through the use of linear aggregation of objective functions. These approaches, although successfully demonstrated, may have limited applicability for complex designs, with non-linear objective functions and a non-convex search space [129]. For example, in the case of FPGA-based implementation of image registration, analytically representing the error induced in MI-calculation will be non-trivial. In addition, MI is a non-linear objective function. Techniques based on evolutionary methods have been shown to be effective in searching large search spaces with complex objective functions in an efficient manner 
[130, 131]. Furthermore, these techniques are inherently capable of performing multipoint searches. As a result, techniques based on evolutionary algorithms (EAs) have been employed in the context of multiobjective optimization (see SPEA2 [160], NSGA-II [161]).

We formulate this problem of finding optimal wordlength configurations as a multiobjective optimization, where different objectives — for example, accuracy and area - generally conflict with one another. Although this approach increases the complexity of the search, it can find a set of Pareto-optimized configurations representing strategically-chosen tradeoffs among the various objectives. This novel multiobjective optimization strategy is developed and validated in the context of FPGA-based implementation of image registration. The tradeoff between FPGA resources (area and memory) and implementation accuracy is systematically explored, and Pareto-optimized solutions are identified such that a designer could use these solutions to satisfy given design constrains or meet image registration accuracy required by an application. This analysis is performed by treating the wordlengths of the internal variables of the architecture presented in the previous chapter as design variables. We also compare several search methods for finding Pareto-optimized solutions and demonstrate in our context the applicability of search based on evolutionary techniques for efficiently identifying superior multiobjective tradeoff curves. This optimization strategy can easily be adapted to a wide range of signal processing applications, including applications for image and video processing. 


\subsection{Multiobjective Optimization}

The architecture presented in the previous chapter is designed to accelerate the calculation of MI for performing intensity-based image registration. We have demonstrated this architecture to be capable of offering execution performance superior to that of a software implementation [157]. The accuracy of MI calculation (and by extension, that of image registration) offered by this implementation, however, is a function of the wordlengths chosen for the internal variables of the design. Similarly, these wordlengths also control the hardware implementation cost of the design. For medical imaging applications, the ability of an implementation to achieve the desired level of accuracy is of paramount importance. It is, therefore, necessary to understand the tradeoff between accuracy and hardware implementation cost for a design and to identify wordlength configurations that provide effective tradeoffs between these conflicting criteria. This multiobjective optimization allows a designer to systematically maximize accuracy for a given hardware cost limitation (imposed by a target device, for example) or minimize hardware resources to meet the accuracy requirements of a medical application.

The following section provides a formal definition of this problem and the subsequent section describes a framework developed for multiobjective optimization of FPGA-based medical image registration.

\subsubsection{Problem Statement}

Consider a system $Q$ that is parameterized by $N$ parameters $n_{i}(i=1,2, \ldots, N)$, where each parameter can take on a single value from a 
corresponding set of valid values $\left(v_{i}\right)$. Let the design configuration space corresponding to this system be $S$, which is defined by a set consisting of all $N$ tuples generated by the Cartesian product of the sets $v_{i}, \forall i$ :

$$
S=v_{1} \times v_{2} \times v_{3} \times \cdots \times v_{N}
$$

The size of this design configuration space is then equal to the cardinality of the set $S$, or in other words, the product of the cardinalities of the sets $v_{i}$ :

$$
|S|=\left|v_{1}\right| \times\left|v_{2}\right| \times\left|v_{3}\right| \times \cdots \times\left|v_{N}\right|
$$

For most systems, not all configurations that belong to $S$ may be valid or practical. We therefore define a subset $\mathfrak{I}(\mathfrak{I} \subseteq S)$, such that it contains all the feasible system configurations. Now consider $m$ objective functions $\left(f_{1}, f_{2}, \ldots, f_{m}\right)$ defined for system $Q$, such that each function associates a real value for every feasible configuration $c \in \mathfrak{J}$.

The problem of multiobjective optimization is then to find a set of solutions that simultaneously optimizes the $m$ objective functions according to an appropriate criterion. The most commonly adopted notion of optimality in multiobjective optimization is that of Pareto optimality. According to this notion, a solution $c^{*}$ is Pareto optimal if there does not exist another solution $c \in \mathfrak{I}$ such that $f_{i}(c) \leq f_{i}\left(c^{*}\right)$, for all $i$, and $f_{j}(c)<f_{j}\left(c^{*}\right)$, for at least one $j$. The solution $c^{*}$ is also called a non-dominated solution, because no other solution dominates (or is superior than) solution $c^{*}$ as per the Pareto-optimality criteria. The set of Pareto optimal solutions, therefore, comprises of all non-dominated solutions. 
Given a multiobjective optimization problem and a heuristic technique for this problem that attempts to derive Pareto-optimal or near-Pareto-optimal solutions, we refer to solutions derived by the heuristic as "Pareto-optimized" solutions.

\subsubsection{Parameterized Architectural Design}

Implementations of signal processing algorithms using microprocessor- or DSP-based approaches are characterized by a fixed datapath width. This width is determined by the hard-wired datapath of the underlying processor architecture. Reconfigurable implementation based on FPGAs, in contrast, allows the size of datapath to be customized to achieve better tradeoffs among accuracy, area, and power. The use of such custom data representation for optimizing designs is one of the main strengths of reconfigurable computing [85]. To take advantage of the described multiobjective optimization strategy, the architecture being optimized must be able to support various design configurations as identified by the optimization scheme. It has even been contended that the most efficient hardware implementation of an algorithm is one that supports a variety of finite precision representations of different sizes for its internal variables [158]. In this spirit, many commercial and research efforts have employed parameterized design style for intellectual property (IP) cores [162-166]. This parameterization capability not only facilitates reuse of design cores, but also allows them to be reconfigured to meet design requirements.

During the design of the aforementioned architecture for accelerated computation of MI, we adopted a similar design style that allows configuration of the wordlengths of the internal variables. Hardware design languages such as VHDL and Verilog natively support hierarchical parameterization of a design through use of 
generics and parameters, respectively. This design style takes advantage of these language features and is employed for the design of all the modules described earlier. We highlight the main features of this design style using illustrative examples and design senarios. Consider a design module with two input variables that computes an output variable through arithmetic manipulation of the input variables. The wordlength of the input variables (denoted by IP1_WIDTH, IP2_WIDTH) and that of the output variable (denoted by OP_WIDTH) are the design parameters for this module. The module can then be parameterized for these design variables as illustrated in Figure 5.1a.

In a pipelined implementation of an operation, a module may have multiple internal pipeline stages and corresponding intermediate variables. Wordlengths chosen for these intermediate variables can also impact the accuracy and hardware requirements of a design. In our implementation scheme, we do not employ any rounding or truncation for the intermediate variables, but deduce their wordlengths based on the wordlengths of the input operands and the arithmetic operation to be implemented. For example, multiplication of two 8-bit variables will, at the most, require a 16-bit wide intermediate output variable. A parameterized implementation of this scenario is illustrated in Figure 5.1c. Sometimes, it is also necessary to instantiate a vendor-provided or a third-party IP core, such as a FIFO module or an arithmetic unit, within a design module. In such cases, we simply pass the wordlength parameters down the design hierarchy to configure the IP core appropriately and thereby maintain the parameterized design style (see Figure 5.1b for example). 
When signals cross module boundaries, the output wordlength and format (position of the binary point) of the source module should match the input wordlength and format of the destination module. This is usually achieved through use of a rounding strategy and right- or left-shifting of the signals. Adopting "rounding toward the nearest” strategy to achieve wordlength-matching is expected to introduce the smallest error, but requires additional logic resources. In our design, we therefore

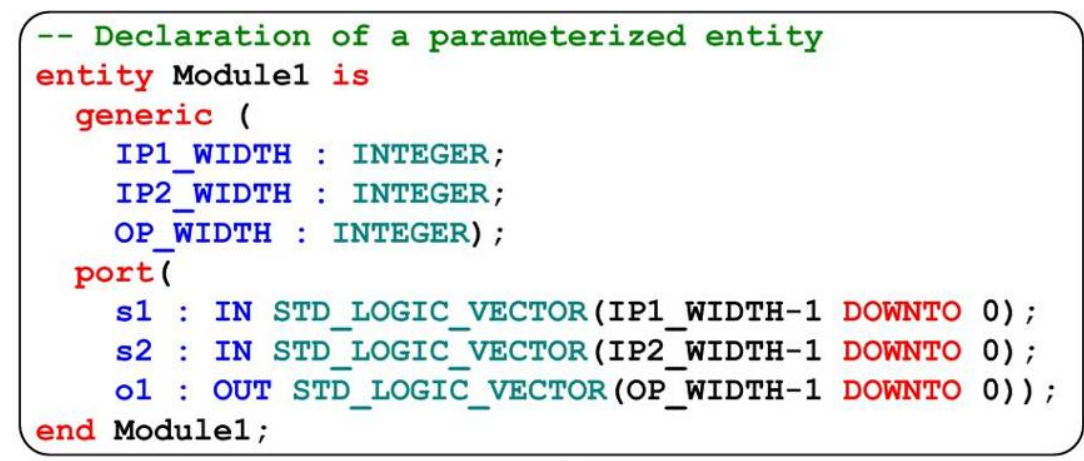

(a)

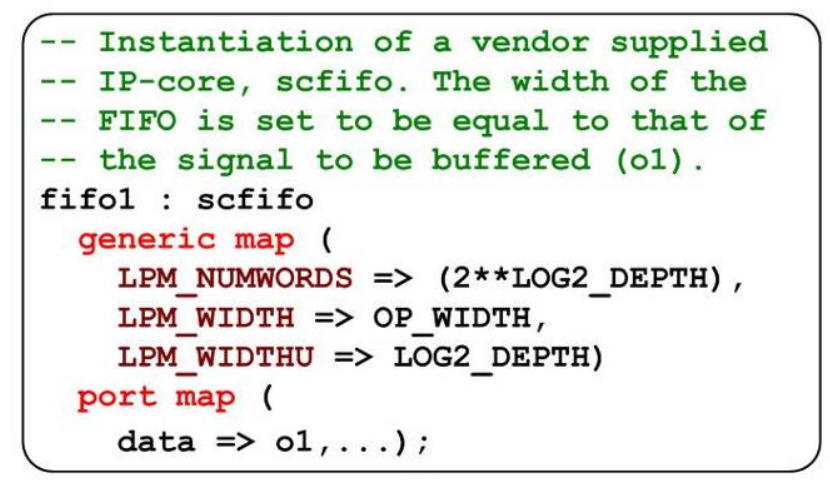

(b)

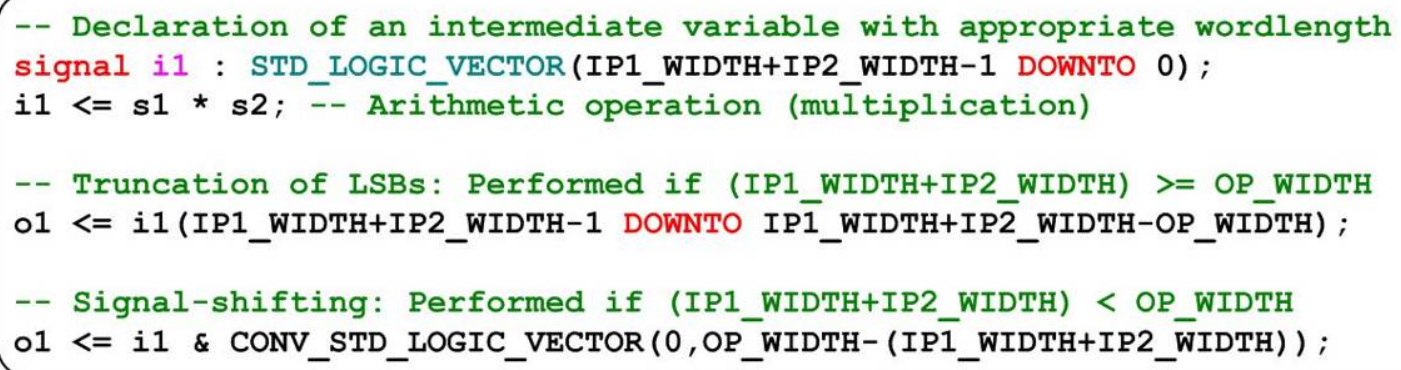

(c)

Figure 5.1: Examples of parameterized architectural design style. 
implement truncation (or "rounding toward zero" strategy), while the signal shifting is achieved through zero-padding. Both these operations are parameterized and take into account the wordlengths and the format at the module boundaries (see Figure 5.1c for example). Thus, this parameterized design style enables the architecture to support multiple wordlength configurations for its internal variables. The parameters of this architecture, and in particular the fractional wordlengths of the internal variables, are being treated as design variables in this multiobjective optimization framework. These design variables are identified in the following section.

\subsubsection{Multiobjective Optimization Framework}

Figure 5.2 illustrates the framework that we have developed for multiobjective optimization of the architecture for high-speed implementation of image registration

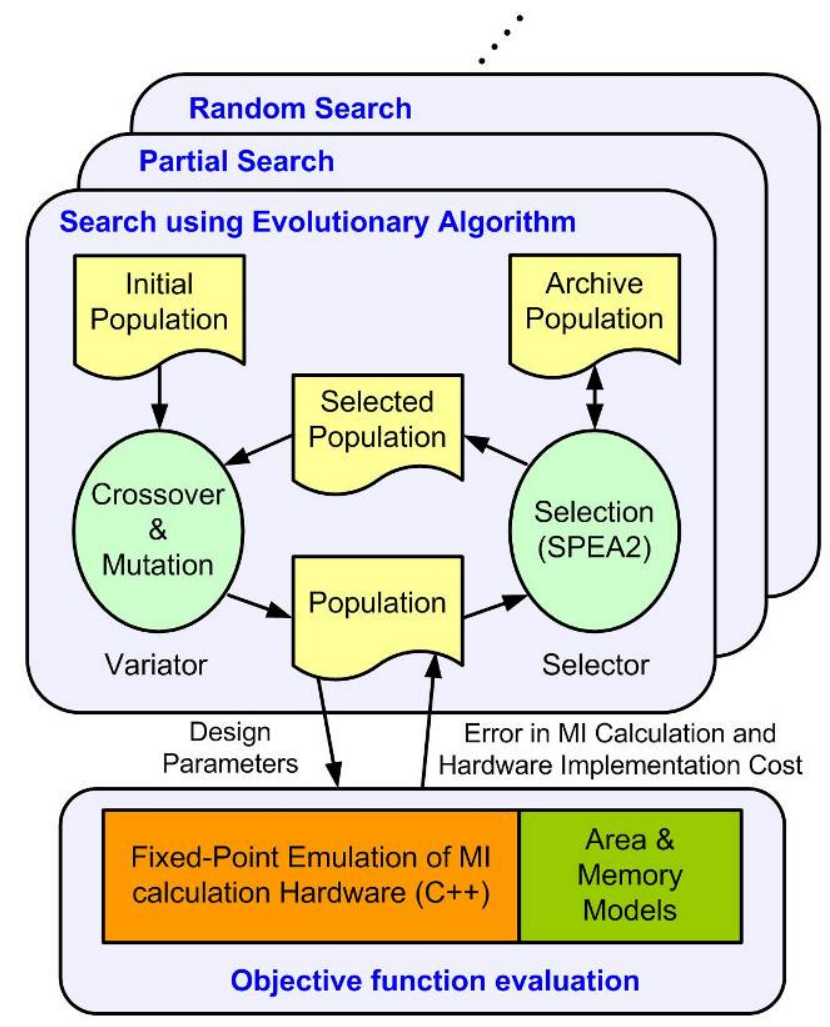

Figure 5.2: Framework for multiobjective optimization of FPGA-based image registration. 
described in the previous chapter. There are two basic components of this framework. The first component is the search algorithm that explores the design space and generates feasible candidate solutions; and the second component is the objective function evaluation module that evaluates candidate solutions. The solutions and associated objective values are fed back to the search algorithm so that they can be used to refine the search. These two components are loosely coupled so that different search algorithms can be easily incorporated into the framework. Moreover, the objective function evaluation module is parallelized using a message passing interface (MPI) on a 32-processor cluster. With this parallel implementation, multiple solutions can be evaluated in parallel, thereby increasing search performance. These components are described in detail in the following sections.

\subsubsection{Design Parameters}

As described in the earlier section, the architecture performs MI calculation using a fixed-point datapath. As a result, the accuracy of MI calculation depends on the precision (wordlength) offered by this datapath. The design parameters in this

datapath define the design space and are identified and listed along with the corresponding design module (see Figure 4.3) in Table 5.1.

A fixed-point representation consists of an integer part and a fractional part. The numbers of bits assigned to these two parts are called the integer wordlength (IWL) and fractional wordlength (FWL), respectively. The individual numbers of bits allocated to these parts control the range and precision of the fixed-point representation. 
Table 5.1: Design variables for FPGA-based architecture. Integer wordlengths are determined based on application-specific range information, and fractional wordlengths are used as parameters in the multiobjective optimization framework.

\begin{tabular}{cccc}
\hline \hline $\begin{array}{c}\text { Architectural } \\
\text { Module }\end{array}$ & $\begin{array}{c}\text { Design } \\
\text { Variable }\end{array}$ & $\begin{array}{c}\text { Integer } \\
\text { wordlength } \\
\text { (IWL ) (bits) }\end{array}$ & $\begin{array}{c}\text { Fractional } \\
\text { wordlength (FWL) } \\
\text { range (bits) }\end{array}$ \\
\hline $\begin{array}{c}\text { Voxel coordinate } \\
\text { transformation }\end{array}$ & Translation vector & 7 & {$[1,32]$} \\
\cline { 2 - 4 } & Rotation matrix & 4 & {$[1,32]$} \\
\hline $\begin{array}{c}\text { Partial volume } \\
\text { interpolation }\end{array}$ & Floating image address & 9 & {$[1,32]$} \\
\hline $\begin{array}{c}\text { Mutual histogram } \\
\text { accumulation }\end{array}$ & $\begin{array}{c}\text { Mutual histogram } \\
\text { bin }\end{array}$ & 25 & {$[1,32]$} \\
\hline \hline
\end{tabular}

$$
\begin{aligned}
\text { Range }(R) & =\left[-2^{I W L}, 2^{I W L}\right) & \ldots & \text { for signed numbers } \\
& =\left[0,2^{I W L}\right) & \ldots & \text { for unsigned numbers }
\end{aligned}
$$

For this architecture, the IWL required for each design parameter can be deduced from the range information specific to the image registration application. For example, in order to support translations in the range of $[-64,63]$ voxels, 7 bits of IWL (with 1 bit assigned as a sign bit) are required for the translation parameter. Similarly, since the current architecture supports images with dimensions up to 512, 9 bits $\left(\log _{2} 512\right)$ of IWL is required for the floating image address. We used similar range information to choose the IWL for all the parameters, and these values are reported in Table 5.1. The precision required for each parameter, which is determined by its FWL, is not known a priori. We, therefore, determine this by performing multiobjective optimization using the FWL of each parameter as a design variable. In our experiments, we used the design range of [1,32] bits for FWLs of all the parameters. The optimization framework can support different wordlength ranges for different parameters, which can be used to account for additional design 
constraints, such as, for example, certain kinds of constraints imposed by third-party intellectual property.

The entropy calculation module is implemented using a multiple-LUT-based approach and also employs fixed-point arithmetic. However, this module has already been optimized for accuracy and hardware resources, as described in [155]. The optimization strategy employed in [155] uses an analytical approach that is specific to entropy calculation and is distinct from the strategy presented in this work. This module, therefore, does not participate in the multiobjective optimization framework presented in this work, and we simply use the optimized configuration identified earlier. This further demonstrates the flexibility of our optimization framework to accommodate arbitrary designer- or externally-optimized modules.

\subsubsection{Search Algorithms}

An exhaustive search that explores the entire design space is guaranteed to find all Pareto-optimal solutions. However, this search can lead to unreasonable execution time, especially when the objective function evaluation is computationally intensive. For example, with four design variables, each taking one of 32 possible values, the design space consists of $32^{4}$ solutions. If the objective function evaluation takes 1 minute per trial (which is quite realistic for multiple MI calculations using large images), the exhaustive search will take 2 years. Consequently, we have considered alternative search methods, as described below.

The first method is partial search, which explores only a portion of the entire design space. For every design variable, the number of possible values it can take is reduced by half by choosing every alternate value. A complete search is then 
performed in this reduced search space. This method, although not exhaustive, can effectively sample the breadth of the design space. The second method is random search, which involves randomly generating a fixed number of feasible solutions. For both of these methods, Pareto-optimized solutions are subsequently identified from the set of solutions explored.

The third method is performing a search using evolutionary techniques. EAs have been shown to be effective in efficiently exploring large search spaces [130, 131]. In particular, we have employed SPEA2 [160], which is very effective in sampling from along an entire Pareto-optimal front and distributing the solutions generated relatively evenly over the optimal tradeoff surface. Moreover, SPEA2 incorporates a fine-grained fitness assignment strategy and an enhanced archive truncation method, which further assist in finding Pareto-optimal solutions. The flow of operations in this search algorithm is shown in Figure 5.2.

For the EA-based search algorithm, the representation of the system configuration is mapped onto a "chromosome" whose "genes" define the wordlength parameters of the system. Each gene, corresponding to the wordlength of a design variable $i$, is represented using an integer allele that can take values from the set $v_{i}$, described earlier. Thus, every gene is confined to wordlength values that are predefined and feasible for a given design variable. The genetic operators for crossover and mutation are also designed to adhere to this constraint and always produce values from set $v_{i}$, for a gene $i$ within a chromosome. This representation scheme is both symmetric and repair-free and, hence, is favored by the schema theory [167], and is computationally efficient, as described in [168]. 


\subsubsection{Objective Function Models and their Fidelity}

Search for Pareto-optimized configurations requires evaluating candidate solutions and determining Pareto-dominance relationships between them. This can be achieved by calculating objective functions for all the candidate solutions and by relative ordering of the solutions with respect to the values of their corresponding objective functions. We consider the error in MI calculation and the hardware implementation cost to be the conflicting objectives that must be minimized for our FPGA implementation problem. We model the FPGA implementation cost using two components: the first is the amount of logic resources (number of LUTs) required by the design, and the second is the internal memory consumed by the design. We treat these as independent objectives in order to explore the synergistic effects between these complementary resources. Because of the size of the design space and limitations due to execution time, it is not practical to synthesize and evaluate each solution. We, therefore, employ models for calculating objective functions to evaluate the solutions. The quality of the Pareto-optimized solutions will then depend on the fidelity of these objective function models.

The error in MI calculation can be computed by comparing the MI value reported by the limited-precision FPGA implementation against that calculated by a double-precision software implementation. For this purpose, we have utilized a bittrue emulator of the hardware. This emulator was developed in $\mathrm{C}++$ and uses fixedpoint arithmetic to accurately represent the behavior of the limited-precision hardware. It supports multiple wordlengths for internal variables and is capable of accurately calculating the MI value corresponding to any feasible configuration. We 
have verified its equivalence with the hardware implementation for a range of configurations and image transformations. This emulator was used to compute the MI calculation error. The MI calculation error was averaged for three distinct image pairs (with different image modality combinations) and for 50 randomly generated image transformations. The same sets of image pairs and image transformations were used for evaluating all feasible configurations.

The memory required for a configuration is primarily needed for intermediate FIFOs, which are used to buffer internal variables, and the MH memory. For example, a 64-word deep FIFO used to buffer a signal with a wordlength of $b$ will require $64 \times b$ bits of memory. In our architecture, the depth of the FIFOs and the dimensions of the $\mathrm{MH}$ are constant, whereas their corresponding widths are determined by the wordlength of the design parameters. Using these insights, we have developed an architecture-specific analytical expression that accurately represents the cumulative amount of memory required for all internal FIFOs and MH. We used this expression to calculate the memory requirement of a configuration.

For estimating the area requirements of a configuration, we adopt the area models reported in $[86,169]$. These are high-level models of common functional units such as adders, multipliers, delays. These models are derived from the knowledge of the internal architecture of these components. Area cost for interconnects and routing is not taken into account in this analysis. These models have been verified for the Xilinx Virtex series of FPGAs and are equally applicable to alternative FPGA families and for ASIC implementations. These models have also been previously used in the context of wordlength optimization [86, 91, 169]. 
We further evaluated the fidelity of these area models using a representative module, PV Interpolator, from the aforementioned architecture. This module receives the fractional components of the floating image address and computes corresponding interpolation weights. We varied the FWL of the floating image address from 1 to 32 bits and synthesized the module using the Altera Stratix II and Xilinx Virtex 5 as target devices. For a meaningful comparison, the settings for the analysis, synthesis, and optimization algorithms (for example, settings to favor area or speed) for the design tools (Altera Quartus II and Xilinx ISE) were chosen to be comparable. After complete synthesis, routing, and placement, we recorded the area (number of LUTs) consumed by the synthesized design. This process was automated by using the Tcl scripting feature provided by the design tools and through the parameterized design style described earlier. We then compared the consumed area against that predicted by the adopted area models for all FWL configurations. The results of this experiment are presented in Figure 5.3. These results indicate that the area estimates (number of

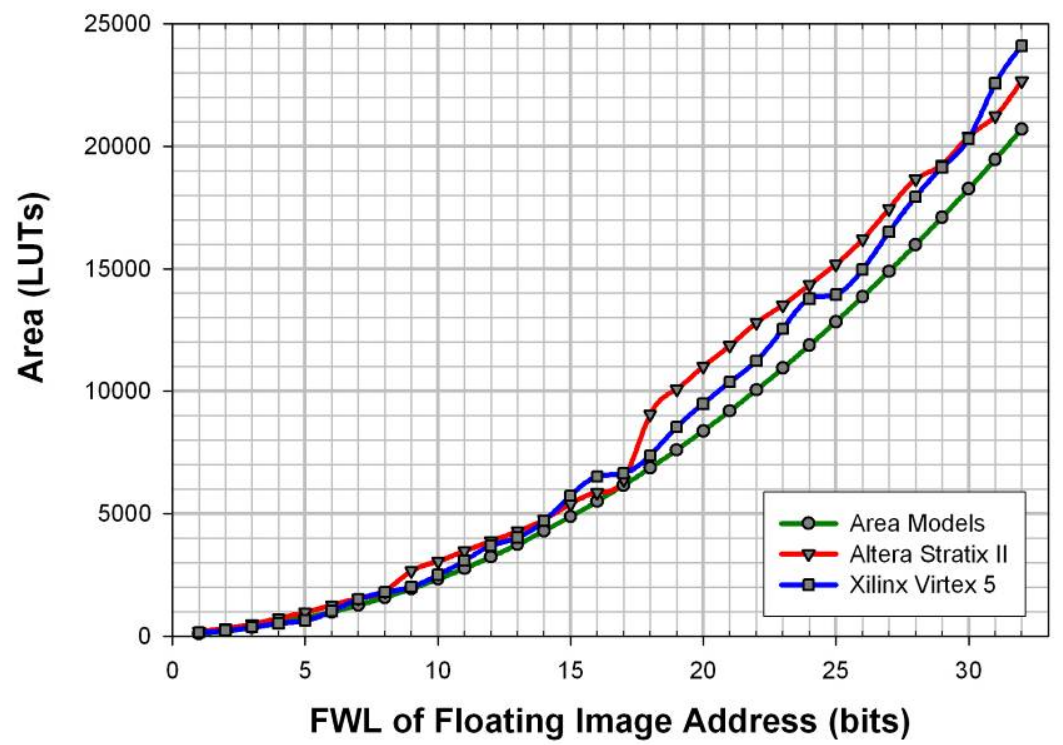

Figure 5.3: Comparison of the area values predicted by the adopted area models with those obtained after physical synthesis. 
LUTs) predicted by the model are comparable to that obtained through physical synthesis for both the target devices. For quantitative evaluation, the fidelity of the area models was calculated as below:

$$
\text { Fidelity }=\frac{2}{N(N-1)}\left(\sum_{i=1}^{N-1} \sum_{j=i+1}^{N} F_{i j}\right),
$$

where

$$
F_{i j}= \begin{cases}1 & \text { if } \operatorname{sign}\left(S_{i}-S_{j}\right)=\operatorname{sign}\left(M_{i}-M_{j}\right) . \\ 0 & \text { otherwise }\end{cases}
$$

In this equation, the $M_{i} \mathrm{~s}$ represent the values predicted by the area models; and the $S_{i} \mathrm{~s}$ represent the values obtained after physical synthesis. The fidelity of the area models when evaluated with respect to the synthesis results obtained for both Altera and Xilinx devices was 1, which corresponds to maximum ("perfect”) fidelity. This indicates that the relative ordering of FWLs with respect to their area requirements is consistent for the model and synthesized designs. These results further validate the applicability of using the aforementioned area models for multiobjective optimization.

\subsection{Experiments and Results}

We performed multiobjective optimization of the aforementioned architecture using the search algorithms outlined in the previous section. To account for the

Table 5.2: N Number of solutions explored by search methods.

\begin{tabular}{cc}
\hline \hline Search Method & $\begin{array}{c}\text { Number of } \\
\text { solutions explored }\end{array}$ \\
\hline Partial search & 65,536 \\
Random search & 6,000 \\
EA-based search & 6,000 \\
\hline \hline
\end{tabular}


Table 5.3: $\quad$ Parameters used for the EA-based search.

\begin{tabular}{cc}
\hline Parameter & Value \\
\hline Population size & 200 \\
Number of generations & 30 \\
Crossover probability & 1.0 \\
Mutation probability & 0.06 \\
\hline \hline
\end{tabular}

effects of random number generation, the EA-based search and random search were repeated five times each, and the average behavior from these repeated trials is reported. The number of solutions explored by each search algorithm in a single run is reported in Table 5.2. The execution time of each search algorithm was roughly proportional to the number of solutions explored, and the objective function evaluation for each solution took approximately 1 minute using a single computing node. As expected, the partial search algorithm explored the largest number of solutions. The parameters used for the EA-based search are listed in Table 5.3. The crossover and mutation operators were chosen to be one-point crossover and flip mutator, respectively. For a fair comparison, the number of solutions explored by the random search algorithm was set to be equal to that explored by the EA-based algorithm.

The solution sets obtained by each search method were then further reduced to corresponding nondominated solution sets using the concept of Pareto optimality. As described earlier, the objectives considered for this evaluation were the MI calculation error and the memory and area requirements of the solutions. Figure 5.4 shows the Pareto-optimized solution set obtained for each search method. Qualitatively, the Pareto front identified by the EA-based search is denser and more widely distributed and demonstrates better diversity than other search methods. 
(a)

Partial Search

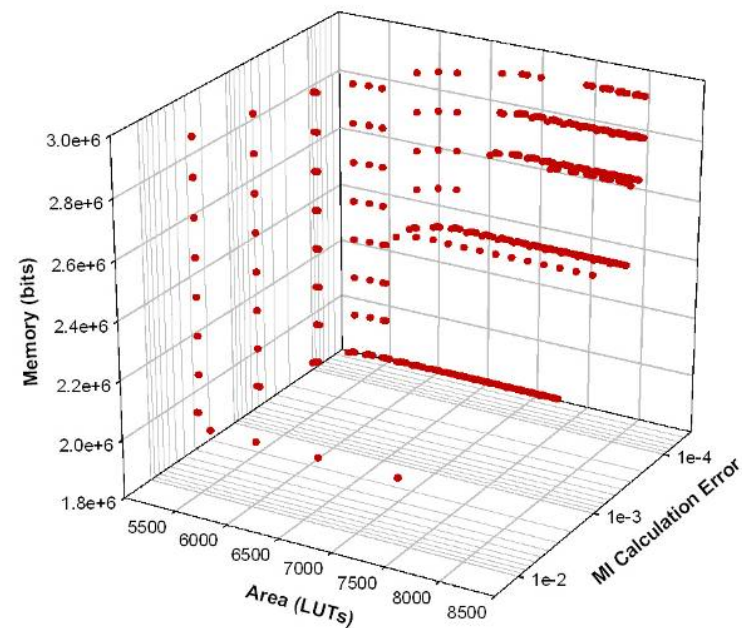

(b)

EA-based Search

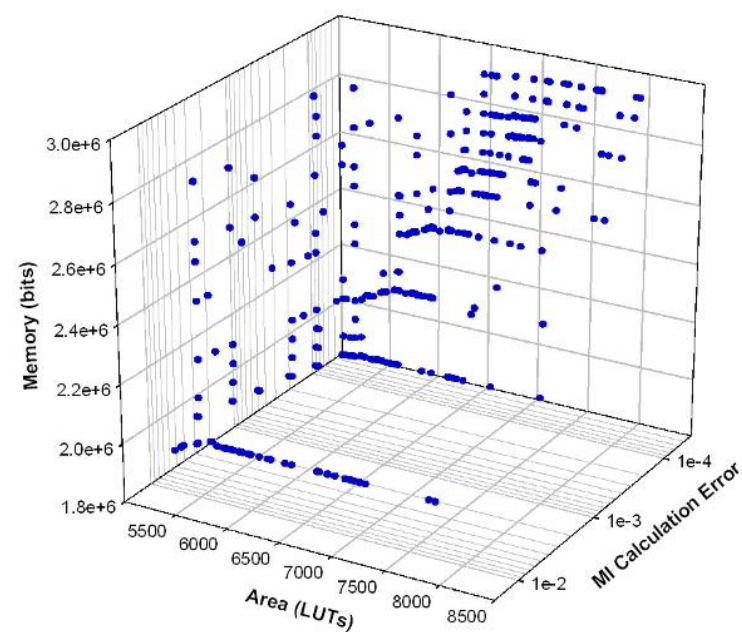

(c)

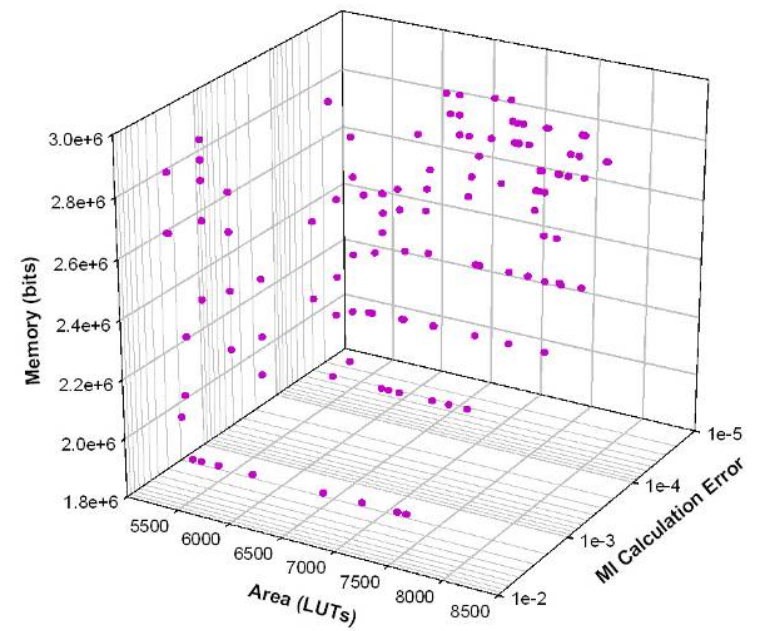

Figure 5.4: $\quad$ Pareto-optimized solutions identified by various search methods. 
Figure 5.5 compares the Pareto fronts obtained by partial search and EA-based search by overlaying them and illustrates that the EA-based search can identify better Paretooptimized solutions, which indicates the superior quality of solutions obtained by this search method. Moreover, it must be noted that the execution time required for the EA-based search was over 10-times faster than that required for the partial search.

(a) Area vs. MI calculation error

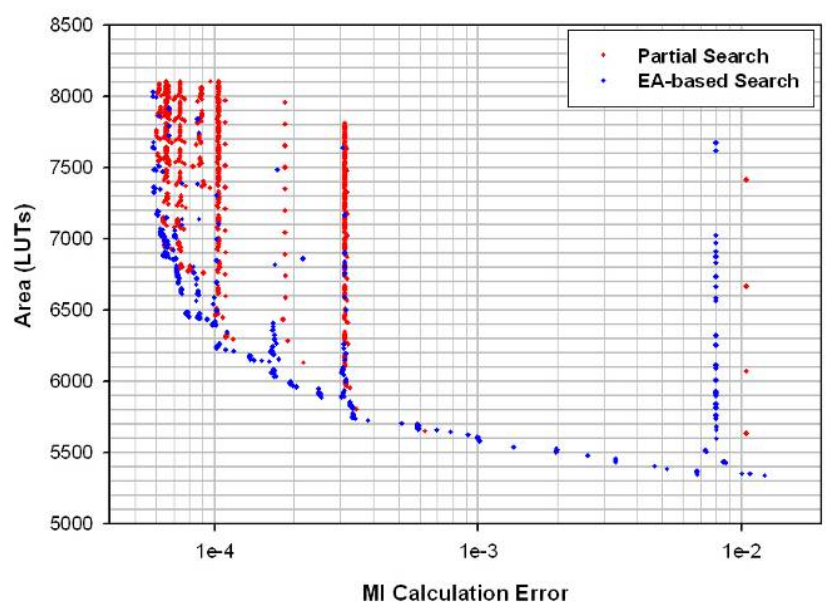

(b) Memory vs. MI calculation error

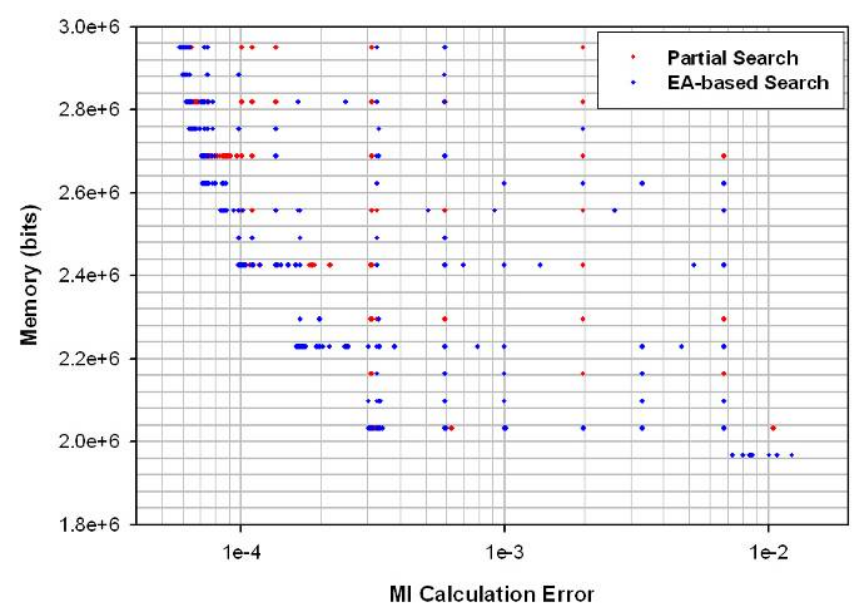

Figure 5.5: Qualitative comparison of solutions found by partial search and EAbased search. 


\subsubsection{Metrics for Comparison of Pareto-optimized Solution Sets}

Quantitative comparison of the Pareto-optimized solution sets is essential in order to compare more precisely the effectiveness of various search methods. As with most real-world complex problems, the Pareto-optimal solution set is unknown for this application. We, therefore, employ the following two metrics to perform quantitative comparison between different solution sets. We use the ratio of nondominated individuals (RNI) to judge the quality of a given solution set, and the diversity of a solution set is measured using the cover rate. These performance measures are similar to those reported in [170] and are described below.

The RNI is a metric that measures how close a solution set is to the Paretooptimal solution set. Consider two solution sets $\left(P_{1}\right.$ and $\left.P_{2}\right)$ that each contain only non-dominated solutions. Let the union of these two sets be $P_{U}$. Furthermore, let $P_{N D}$ be a set of all non-dominated solutions in $P_{U}\left(P_{N D} \subseteq P_{U}\right)$. The RNI for the solution set $P_{i}$ is then calculated as:

$$
R N I_{i}=\frac{\left|P_{i} \cap P_{N D}\right|}{\left|P_{N D}\right|},
$$

where $|\cdot|$ is the cardinality of a set. The closer this ratio is to $100 \%$, the more superior the solution set is and the closer it is to the Pareto-optimal front. We computed this metric for all the search algorithms previously described, and the results are presented in Figure 5.6. Our EA-based search offers better RNI and, hence, superior quality solutions to those achieve7d with either the partial or random search. 

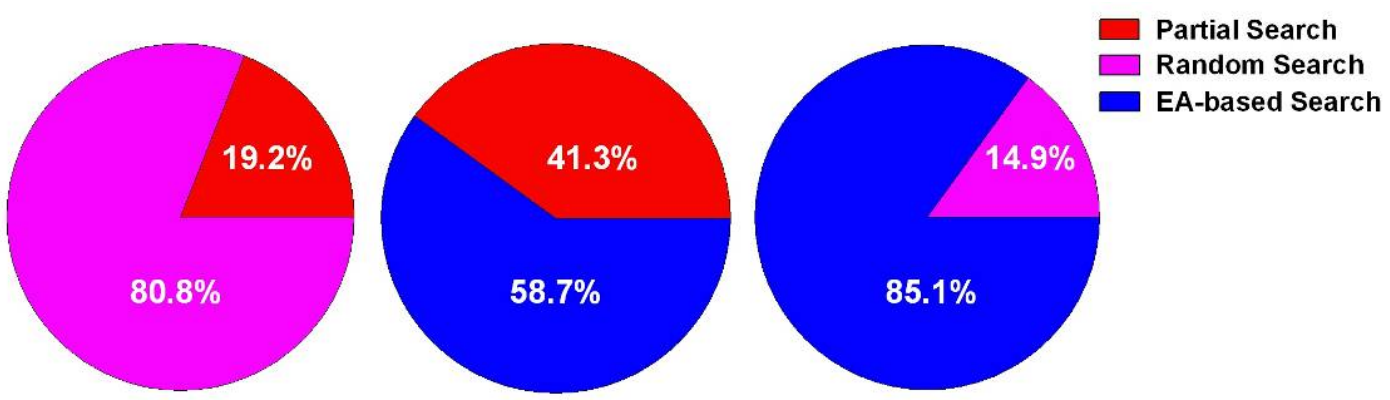

Figure 5.6: Quantitative comparison of search methods using the ratio of nondominated individuals (RNI).

The cover rate estimates the spread and distribution (or diversity) of a solution set in the objective space. Consider the region between the minimum and maximum of an objective function as being divided into an arbitrary number of partitions. The cover rate is then calculated as the ratio of the number of partitions that is covered (that is, there exists at least one solution with an objective value that falls within a given partition) by a solution set to the total number of partitions. The cover rate $\left(C_{k}\right)$ of a solution set for an objective function $\left(f_{k}\right)$ can then be calculated as:

$$
C_{k}=\frac{N_{k}}{N},
$$

where $N_{k}$ is the number of covered partitions and $N$ is the total number of partitions. If there are multiple objective functions ( $m$, for example), then the net cover rate can be obtained by averaging the cover rates for each objective function as:

$$
C=\frac{1}{m} \sum_{k=1}^{m} C_{k} .
$$

The maximum cover rate is 1 , and the minimum value is 0 . The closer the cover rate of a solution set is to 1 , the better coverage and more even (more diverse) distribution it has. Because the Pareto-optimal front is unknown for our targeted application, the 


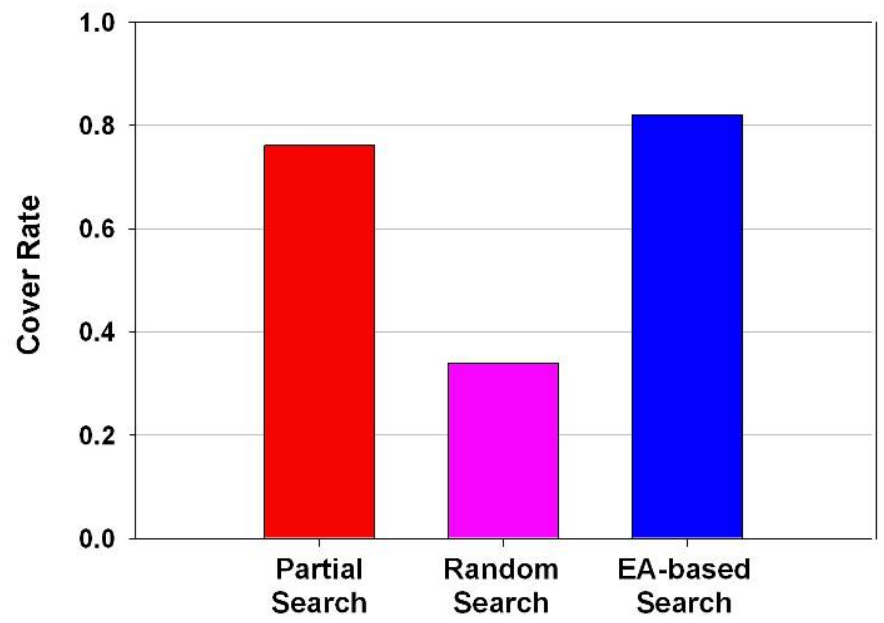

Figure 5.7: Quantitative comparison of search methods using cover rate.

minimum and maximum values for each objective function were selected from the solutions identified by all the search methods. We used 20 partitions/decade for MI calculation error (represented using a logarithmic scale), 1 partition for every 50 LUTs for the area requirement, and 1 partition for every 50 Kbits of memory requirement. The cover rate for all the search algorithms described earlier was calculated using the method outlined above, and the results are illustrated in Figure 5.7. The EA-based search offers a better cover rate, which translates to better range and diversity of solutions when compared with either partial or random searches. In summary, our EA-based search outperforms the random search and is capable of offering more diverse and superior quality solutions when compared with the partial search, using only $10 \%$ of the execution time.

\subsubsection{Accuracy of Image Registration}

An important performance measure for any image registration algorithm, especially in the context of medical imaging, is its accuracy. We did not choose 


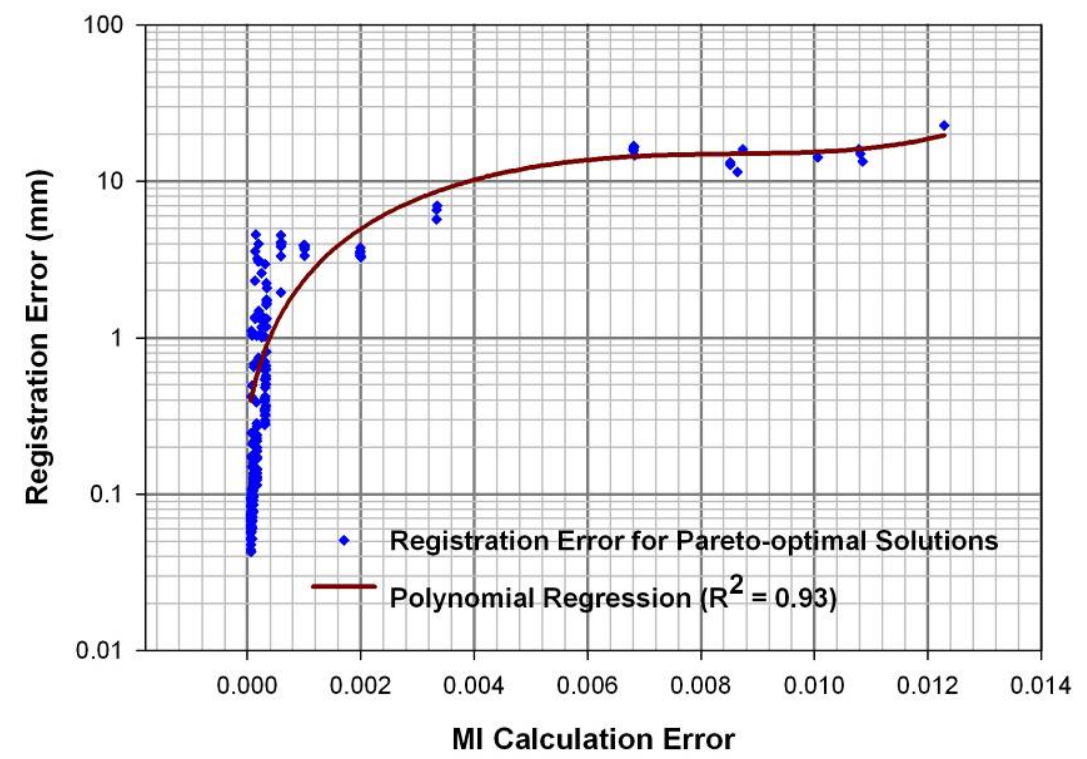

Figure 5.8: Relationship between MI calculation error and resulting image registration error.

registration accuracy as an objective function because of its dependence on data (image pairs), the degree of misalignment between images, and the behavior of the optimization algorithm that is used for image registration. These factors, along with its execution time, in our experience, may render registration accuracy as an unsuitable objective function, especially if there is non-monotonic behavior with respect to the wordlength of design variables.

Instead, we evaluated the affect of error in MI calculation on the image registration accuracy for a set of image pairs. This analysis was performed using three computed tomography image pairs for the Pareto-optimized solutions identified by all of the search algorithms that we experimented with. Image registration was performed using limited-precision configurations corresponding to each solution using the aforementioned bit-true simulator. The result of registration was then compared with that obtained using double-precision software implementation. Registration accuracy was calculated by comparing deformations at the vertices of a 
cuboid (with size equal to half the image dimensions) located at the center of the image. The results of this analysis are illustrated in Figure 5.8. As expected, there is a good correlation between the MI calculation error and the accuracy of image registration. This demonstrates that optimized tradeoff curves between MI calculation error and hardware cost, as identified by our reported analysis, can be used to represent the relationships between registration accuracy and hardware cost with high fidelity. This analysis also provides better insight about the sensitivity of image registration accuracy to various design parameters.

\subsubsection{Post-synthesis Validation}

We performed further validation of the presented multiobjective optimization strategy through physical design synthesis. We identified three solutions from the Pareto-optimized set obtained using the EA-based search and synthesized the aforementioned architecture with configurations corresponding to these solutions. These three configurations, which offer gradual tradeoff between hardware resource requirement and error in MI calculation, are listed in the first column of Table 5.4. The wordlengths associated with each configuration correspond to the FWLs of the design variables identified in Table 5.1. The design was synthesized for these configurations and the resulting realizations were implemented using an Altera Stratix II EP2S180F1508C4 FPGA (Altera Corporation, San Jose, CA) on a PCI prototyping board (DN7000K10PCI) manufactured by the Dini Group (La Jolla, CA). We then evaluated the performance of the synthesized designs and compared it with that predicted by the objective function models. The results of this analysis are summarized in Table 5.4 and are described below. 
Table 5.4: Validation of the objective function models using post-synthesis results. The wordlengths in a design configuration correspond to the FWLs of the design variables identified earlier.

\begin{tabular}{ccccc}
\hline \hline Design & \multicolumn{3}{c}{$\begin{array}{c}\text { Objective Functions } \\
\text { Post-Synthesis Value } \\
\text { (Predicted Value) }\end{array}$} & $\begin{array}{c}\text { Image } \\
\text { Registration } \\
\text { Error } \\
\text { (mm) }\end{array}$ \\
\cline { 2 - 4 } & $\begin{array}{c}\text { MI Calculation } \\
\text { Error }\end{array}$ & $\begin{array}{c}\text { Area } \\
\text { (No. of LUTs) }\end{array}$ & $\begin{array}{c}\text { Memory } \\
\text { (Mbits) }\end{array}$ & \\
\hline \multirow{2}{*}{$5,6,4,9$} & $2.4 \times 10^{-3}$ & 6527 & 2.23 & \multirow{2}{*}{3.82} \\
& $\left(2.1 \times 10^{-3}\right)$ & $(5899)$ & $(2.23)$ & \\
$\{8,9,7,12\}$ & $5.3 \times 10^{-4}$ & 7612 & 2.45 & 1.57 \\
$\{9,12,10,17\}$ & $\left(5.2 \times 10^{-4}\right)$ & $(6754)$ & $(2.45)$ & \\
\hline \hline
\end{tabular}

The error in MI calculation was computed by comparing the MI value reported by the limited-precision FPGA implementation against that calculated by a double-precision software implementation. The MI calculation error was averaged for three distinct image pairs and for 50 randomly generated image transformations for each pair. These image pairs and the associated transformations were identical to those employed in the objective function calculation. In this case, the average MI calculation error obtained by all the design configurations was identical to that predicted by the objective function model. This is expected due to the bit-true nature of the simulator used to predict the MI calculation error. We repeated this calculation with a different set of three image pairs and 50 randomly generated new transformations associated with each image pair. The MI calculation error corresponding to this setup is reported in the second column of Table 5.4. The small difference when compared to the error predicted by the models is explained by the different sets of images and transformations used. The area and memory requirement corresponding to each configuration after synthesis are reported in columns three and 
four of Table 5.4, respectively. For comparison, we have also included the values predicted by the corresponding objective function models in parenthesis. It must be noted that for all the three configurations, the relative ordering based on Paretodominance relationships with respect to each objective function is identical for both post-synthesis and model-predicted values.

We also evaluated the accuracy of image registration performed using the implementation corresponding to each design configuration. For this analysis, we considered five computed tomography image pairs. The image registration results for a representative image-pair are illustrated in Figure 5.9. Subfigures (a) and (b) show two distinct poses for the same subject; and (c) shows fusion of (a) and (b) using a checkerboard pattern. The misalignment between images is evident at the edges of the squares within the checkerboard pattern. Subfigures (d)-(f) show fusion images after registration using the identified design configurations. These configurations offer progressively reducing image registration error $(3.82 \mathrm{~mm}, 1.57 \mathrm{~mm}$, and $0.45 \mathrm{~mm}$, respectively), and result into correspondingly improved image alignment. The arrows indicate representative regions with misalignment that are better-aligned after registration. The registration error was calculated by comparing the obtained registration results with that obtained using double-precision software implementation. The average registration error for each configuration is reported in the last column of Table 5.4. There is a good correlation between the MI calculation error and the registration error, reinforcing the results presented in the previous section. 


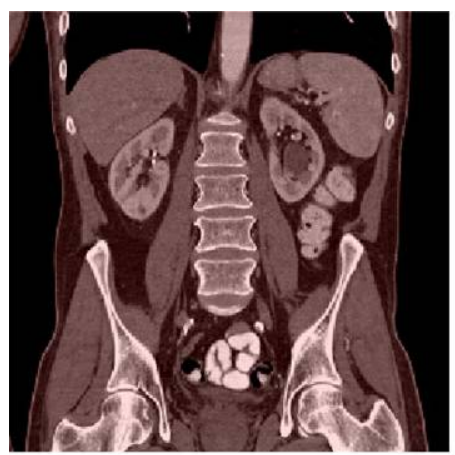

(a)

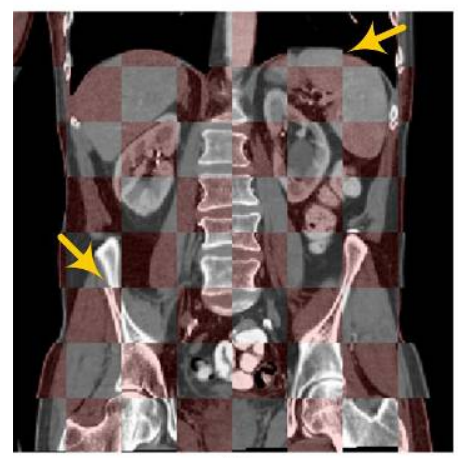

(d)

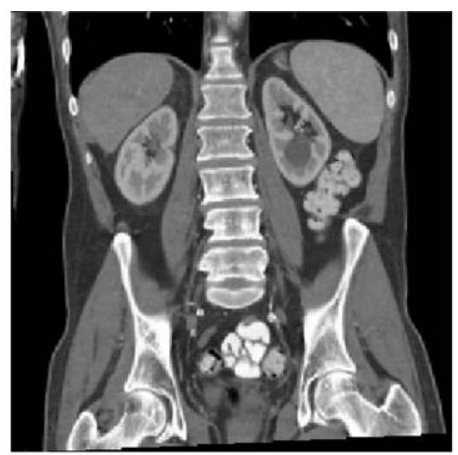

(b)

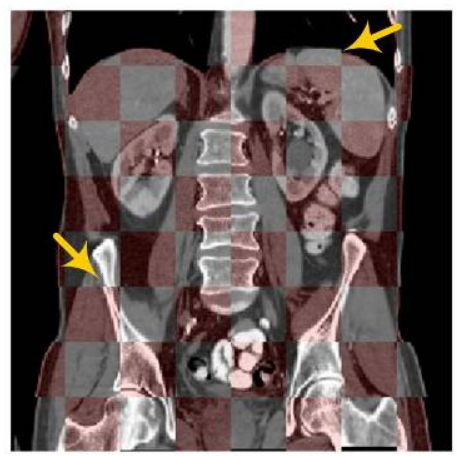

(e)

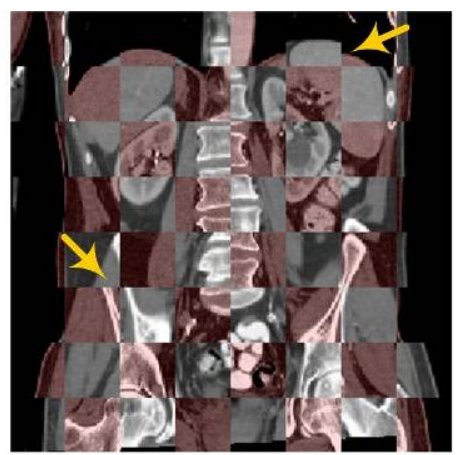

(c)

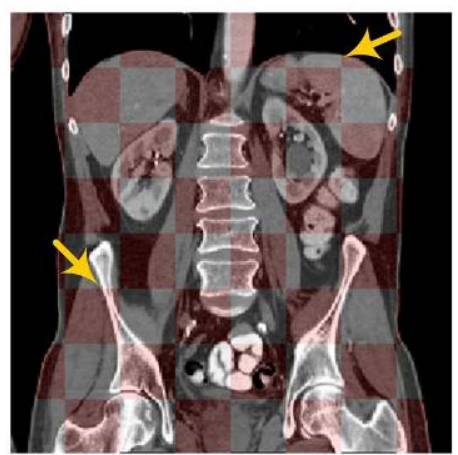

(f)

Figure 5.9: Results of image registration performed using the high-speed, FPGAbased implementation for design configurations offering various registration errors.

This post-synthesis validation further demonstrates the efficacy of the presented optimization approach for reconfigurable implementation of image registration. It also further demonstrates how the approach enables a designer to systematically choose an efficient system configuration to meet the registration accuracy requirements for a reconfigurable implementation.

As described previously, we used error in calculation of MI as one of the objective functions. The Pareto-optimized solutions identified by the search schemes employed in this multiobjective optimization framework can then be used to select a design configurations that offer best (lowest) error in calculation of MI for various hardware resource requirements. Through additional experiments, both simulationbased and post-synthesis validation-based, we have further demonstrated that there is 
a good correlation between error in MI calculation and the image registration error. In general, reducing the error in the calculation of MI will translate into improved image registration accuracy. This is expected, since MI is used as a similarity measure for performing image registration. Although, the image registration algorithm being accelerated in the current work is of deformable nature, it achieves deformable alignment through a series of hierarchical, locally rigid registrations that use MI as a similarity measure. We, therefore, contend that the accuracy of the deformable registration is directly dependant on and highly correlated with the accuracy of MI calculation. Consequently, the design configuration offering better accuracy (lower error) in MI calculation will lead to superior image registration accuracy. This is also supported by the data presented in Table 5.4. As accuracy of image registration can play a crucial rule in IGI applications (and other medical applications, in general), we selected the system configuration that offered lowest error in MI calculation (and by extension lowest image registration error) from Table 5.4. This system configuration $(\{9,12,10,17\})$ was then used for validation of deformable registration in the context of novel IGI applications described in the following chapter.

\subsection{Summary}

One of the main strengths of reconfigurable architectures over generalpurpose processor-based implementations is their ability to utilize more streamlined representations for internal variables. This ability can often lead to superior performance, as demonstrated by our architectures (for performing 3D image preprocessing and deformable image registration) presented in the previous chapters and by other researchers in the context of myriad of other applications. Furthermore, 
this approach can result into optimized utilization of FPGA resources, by employing just enough precision for each of its internal variables to satisfy design requirements of a given application. Given this advantage, it is highly desirable to automate the derivation of optimized design configurations that offer varying degree of tradeoff between implementation accuracy and hardware resources. Toward that end, this work has presented a framework for multiobjective optimization of finite precision, reconfigurable implementations. This framework considers multiple conflicting objectives, such as hardware resource consumption and implementation accuracy, and systematically explores tradeoff relationships among the targeted objectives. This work has also further demonstrated the applicability of EA-based techniques for efficiently identifying Pareto-optimized tradeoff relations in the presence of complex and non-linear objective functions. The evaluation that we have performed in the context of the architecture for FPGA-based deformable image registration demonstrates that such an analysis can be used to enhance automated hardware design processes, and efficiently identify a system configuration that meets given design constraints. This approach may also be applied in the context of reconfigurable computing for identifying suitable design configurations that can be switched among at runtime. Furthermore, the multiobjective optimization approach that we have presented is quite general, and can be extended to a multitude of other signal processing applications. 


\section{Chapter 6: Clinical Applications}

Earlier chapters of this dissertation have identified, described, and optimized the core components of an advanced image-guidance system for IGI applications. These components, namely real-time image processing and high-speed deformable image registration, enable the use of 3D image processing in the IGI workflow. This workflow is illustrated pictorially in Figure 6.1. For this example, we considered CT to be the intraprocedural imaging modality (labeled as iCT) and PET to be the preprocedural image modality (labeled as PET). However, it must be noted, that this workflow is only representative and can be extended to incorporate multiple image modality combinations (such as CT and contrast-enhanced CT, or MRI, etc.) for various IGI applications. This chapter validates the high-speed implementation of deformable image registration and demonstrates the feasibility of using these components to improve existing procedures and enable development of novel imageguided applications. We consider two such clinical applications. First, we propose a strategy for intraprocedural radiation dose reduction in the context of CT-guided

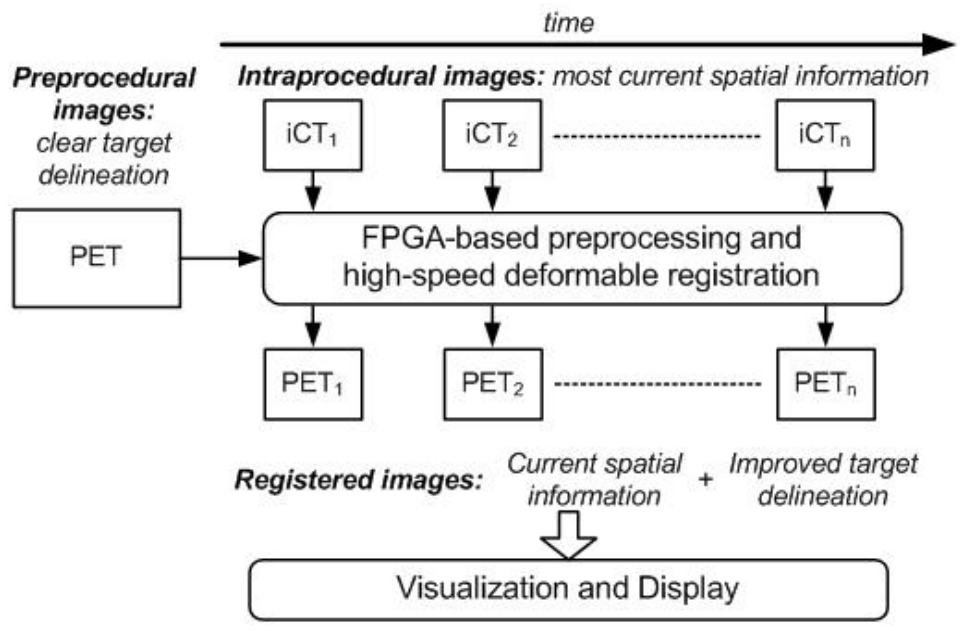

Figure 6.1: Integration of deformable registration into IGI workflow. 
procedures. Second, we demonstrate the feasibility of incorporating PET into liver radiofrequency ablations, a common procedure for treating liver tumors.

\subsection{Radiation Dose Reduction}

\subsubsection{Motivation}

$3 \mathrm{D}$ visualization is a critical need of image guided interventions. To perform true 3D visualization, a volumetric image of the operative field is essential, the type of data which is native to modern CT/MRI, but not to 2D-ultrasound or fluoroscopic imaging which are conventionally used for image-guided interventions [18, 20, 21] . Continuous real-time 3D imaging in an interventional suite is the first step to equip interventionists with enhanced visualization capabilities. However, continuous 3D imaging has been technologically difficult until recently. MRI remains slow and while real-time 3D ultrasonography was recently released, its image quality remains suboptimal compared with that of CT and MRI. Recently introduced multi-slice CT does not suffer from these limitations and can, in fact, image the operative field at a high resolution and a very high frame rate (sub-seconds). As a result of this, many interventional procedures such as liver biopsies, cryo-and radio-frequency ablations are now being routinely carried out under volumetric CT-guidance [171-173]. With time, its speed, image resolution and coverage will only improve, making it even more desirable for live intraprocedural imaging. Several imaging equipment manufacturers, Toshiba and Philips, for example, have announced availability of 256slice scanners $[25,174]$ providing sufficient coverage $(8-12 \mathrm{~cm})$ for most image guided interventions. Radiation exposure to the patient and the interventionist, 
however, continues to be a concern with use of continuous or on-demand CT. It is, therefore, necessary to acquire the intraprocedural images at a lower dose, such that the net radiation dose is within the safe limits. There are some previous studies which have reported use of low-dose CT [175-177], however, these techniques were primarily employed for diagnostic and computer-aided detection applications and did not involve image registration.

\subsubsection{Dose Reduction Strategy}

Our primary radiation dose reduction strategy is to acquire a standard-dose preprocedural CT image and scan the dynamic operative field subsequently using low-dose CT. Using the high-speed deformable registration technique described earlier, the preprocedural CT image can then be registered to low-dose intraprocedural CT images. Registered preprocedural CT will then show the dynamic intraprocedural anatomy and will substitute the low-dose CT images. These diagnostic quality images can then be $3 \mathrm{D}$ rendered and used for intraprocedural guidance and navigation. Capability of viewing hidden vasculature using preprocedural contrast-enhanced CT together with the additional capability of virtually inserting intraprocedural tools in the 3D renderings will add a new dimension to CT-guided interventions.

This proposed dose reduction strategy necessitates the determination of the threshold radiation dose for intraprocedural CT, which permits its accurate image registration with preprocedural CT. The following sections describe a preliminary study to evaluate the registration accuracy with low-dose CT. 


\subsubsection{Evaluation of Registration Accuracy with Low-Dose CT}

Quantifying the accuracy of deformable registration, in general, is a very difficult task due to the lack of a well known gold standard. It is, however, necessary to judge the registration accuracy in the proposed application to determine the optimal radiation dose that does not sacrifice the precision of an image-guided procedure. Since our images are to be registered in the present case, our validation strategy has been to test how well the deformable registration algorithm recovers a userintroduced, known non-rigid misalignment. Our overall strategy can then be described in following main steps: 1) Generate images representing the same anatomy at varying radiation doses, 2) introduce the same known deformation in lowdose CT images, 3) preprocess the deformed low-dose CT images to improve SNR prior to the registration, 4) register the preprocedural standard-dose image with the deformed intraprocedural (simulated) low-dose images using deformable image

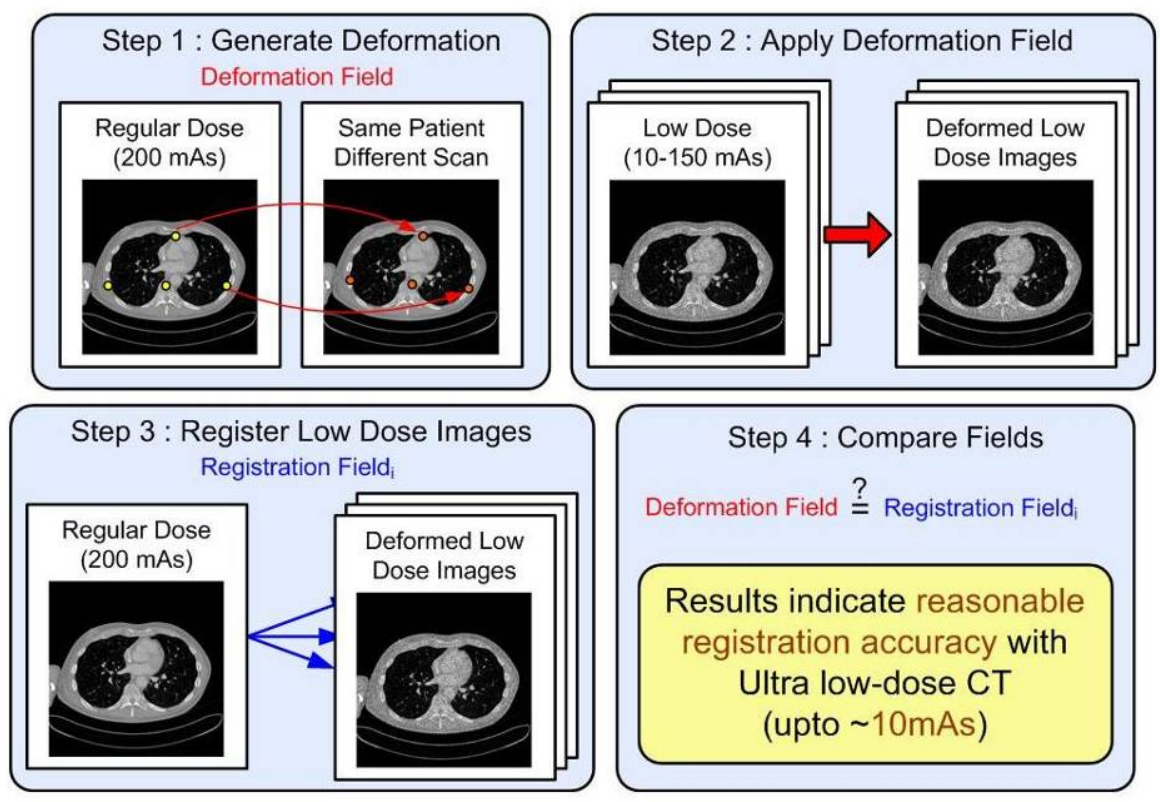

Figure 6.2: Important steps for evaluating registration accuracy with low-dose CT. 
registration and finally, 5) compare the transformation field obtained after image registration with the original, user-introduced deformation field to calculate the registration accuracy at various doses. The important steps in this workflow are illustrated in Figure 6.2 and are described below.

\subsubsection{Generating Low-Dose CT Images}

Low dose images corresponding to a standard dose abdominal scan were generated using syngo-based Somaris/5 simulator from Siemens. This simulator models the noise and attenuation effects at lower radiation doses and can generate low-dose equivalent images from tomographic projection data corresponding to an input standard-dose image. The performance and accuracy of this simulator has been previously reported [178]. This approach ensures that scans at all radiation doses represent exactly the same anatomy. Example low-dose images generated using this simulator are shown in Figure 6.3. Subfigure (a) shows a coronal slice for an input image at a standard dose (200 mAs), and subfigures (b-d) show the corresponding coronal slice for the low-dose images generated using the dose simulator at various doses.

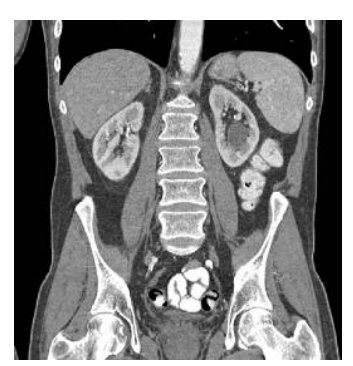

a) $200 \mathrm{mAs}$

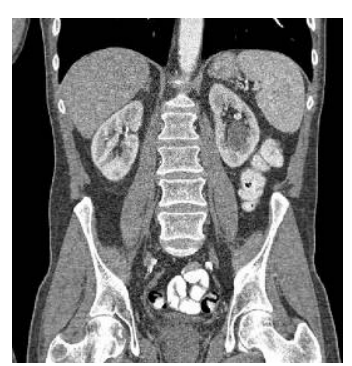

b) $50 \mathrm{mAs}$

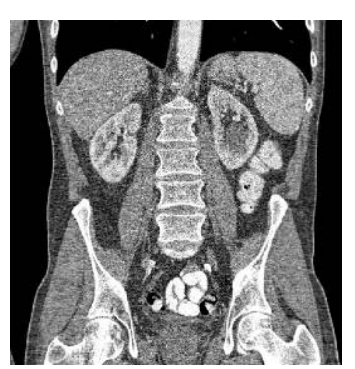

c) $20 \mathrm{mAs}$

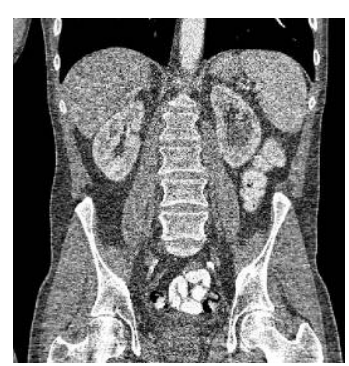

d) $10 \mathrm{mAs}$

Figure 6.3: Low-dose CT images generated by the dose-simulator. 


\subsubsection{Creating Anatomically Realistic Deformations}

Human body, and abdominal organs and tissues in particular, undergo nonrigid deformation during day-to-day activities, respiratory and cardiac cycles, etc. These deformations manifest as misalignment between preprocedural and intraprocedural scans. Further misalignments are introduced due to differences in patient position during imaging as well as different scan parameter settings. In order to create a realistic deformation that incorporates all these effects, it is necessary to estimate this deformation from scans of the same anatomy taken on different days, thus ensuring sufficient temporal separation.

We, therefore, consider CT images for the same subject acquired at different times as a suitable image-pair. We then identify several significant anatomical landmarks from both these images. Deformation vectors between homologous anatomical landmarks in such two images represent the local misalignment at those landmarks. Using these vectors, deformation field for the entire anatomy can then approximated using thin-plate spline (TPS)-based interpolation [179]. TPS is an

interpolation scheme based on radial basis functions and is capable of providing smooth 2D or 3D interpolation for non-uniformly spaced sample points. This technique has been applied extensively for various medical imaging applications $[180,181]$.

\subsubsection{Image Preprocessing}

Ultra low-dose CT scans acquired during the procedure show the exact same anatomy as a standard dose scan would but are characterized by high level of quantum noise. These scans may not be acceptable for diagnostic purposes, but 
contain sufficient information regarding the current anatomical state. From the perspective of MI-based deformable image registration, using these low-dose images as is will cause the dispersion of the mutual histogram (due to noise, in an otherwise uniform structure) leading to poor image registration. Anisotropic diffusion filtering has been shown to be an effective processing step prior to advanced image processing [76, 83, 182, 183]. Figure 6.4 compares the performance of anisotropic diffusion filtering against other standard preprocessing techniques. Higher value of mutual information between the original and the filtered image after preprocessing, which represents increased structural similarity, indicates superior filtering performance for a given pair of images. We, therefore, enhance the low-dose CT images through use of anisotropic diffusion filtering prior to image registration. Anisotropic diffusion filtering, however, is an iterative process which can take up to minutes on a modern CPU. The FPGA-based real-time implementation of this operation, which has been described earlier, can be employed to accelerate the execution of this step.

\subsubsection{Applying Deformation and Image Registration}

The deformation field generated using TPS-based interpolation is applied to the low-dose images for all the simulated doses. This involves resampling of the low-

\begin{tabular}{|c|c|c|c|c|}
\hline $\begin{array}{c}\text { Standard Dose } \\
(200 \mathrm{mAs})\end{array}$ & $\begin{array}{c}\text { Ultra Low Dose } \\
(10 \mathrm{mAs})\end{array}$ & $\begin{array}{c}\text { Median } \\
\text { Filtered }\end{array}$ & $\begin{array}{c}\text { Gaussian } \\
\text { Filtered }\end{array}$ & $\begin{array}{c}\text { Anisotropic } \\
\text { Diffusion Filtered }\end{array}$ \\
\hline & & & \\
\hline
\end{tabular}

Figure 6.4: Comparison of techniques for preprocessing low-dose CT images. 
dose image on to a regular grid using the anatomical mapping provided by the TPSgenerated deformation field. The image registration between these deformed lowdose images and the original standard-dose image yields a registration field, which attempts to estimate and recover the induced anatomically realistic deformation. The mean-squared difference between the deformation vectors at every voxel corresponding to the registration field and the induced deformation field provides a measure of registration accuracy at a particular radiation dose.

\subsubsection{Experiments}

An abdominal scan acquired under clinical settings at the standard dose (200 mAs) was selected for this study. Dose correction feature of the scanner, which automatically modulates the radiation dose based on the anatomy to be scanned, was turned off for this scan to keep the dose consistent across all the slices. The CT image acquired measured $256 \times 256 \times 300$ voxels, with voxel dimensions of $1.56 \mathrm{~mm} \times 1.56$ $\mathrm{mm} \times 1.5 \mathrm{~mm}$ and field of view restricted mostly to lower thorax and abdomen.

Four different CT scans of the same subject acquired at different times within a span of one to sixty days were used for creating anatomically realistic deformations. These prior scans had dimensions and resolution of $256 \times 256 \times 280-315$ and 1.48 $\mathrm{mm} \times 1.48 \mathrm{~mm} \times 1.5 \mathrm{~mm}$ respectively. Based on a predetermined, well-described list of 32 anatomical landmarks, a clinical expert identified and marked a set of homologous points in all the CT scans (one standard-dose scan and four older scans). Based on the expert-defined landmarks, TPS-based starting deformation fields $\left(\mathrm{DF}_{1}, \mathrm{DF}_{2}, \mathrm{DF}_{3}\right.$, and $\mathrm{DF}_{4}$ ) corresponding to each prior scans were generated. 
Treating the standard-dose scan as a reference, we generated eleven low-dose scans (at 10, 15, 20, 25, 30, 40, 50, 70, 85, 100, $150 \mathrm{mAs}$ ) using the Somaris/5 simulator. 10mAs was the lowest setting possible for the current version of the simulator. Each of these scans (including the standard dose scan) was deformed using the realistic deformation fields $\mathrm{DF}_{1}, \mathrm{DF}_{2}, \mathrm{DF}_{3}$, and $\mathrm{DF}_{4}$ described above.

We registered these deformed and preprocessed low-dose images (reference image) with the original standard-dose image (floating image) using the deformable registration algorithm and its accelerated implementation described earlier. Alignment between the floating and reference after deformable image registration yields a registration field $\left(\mathrm{RF}_{\mathrm{i}}\right)$ which maps each reference image voxel into floating image coordinate space. Comparison of this registration field $\left(\mathrm{RF}_{\mathrm{i}}\right)$ with the originally introduced deformation field $\left(\mathrm{DF}_{\mathrm{j}}\right)$ was used to judge the registration accuracy for each dose.

\subsubsection{Results}

Result of the deformable registration using the hardware implementation of the registration algorithm for a representative image-pair generated using one of the deformations $\left(\mathrm{DF}_{1}\right)$ is shown in Figure 6.5. Columns a) and b) show the two starting poses, and column c) the starting pose difference that the image registration must recover. The difference images after image registration at various CT doses are shown in columns d) to f). The top row shows coronal slices of the image and the corresponding axial slices are shown in the bottom row. The software implementation produced qualitatively similar registration results. Visually correct registration of the standard-dose image with the deformed images at various low doses (evident from the 


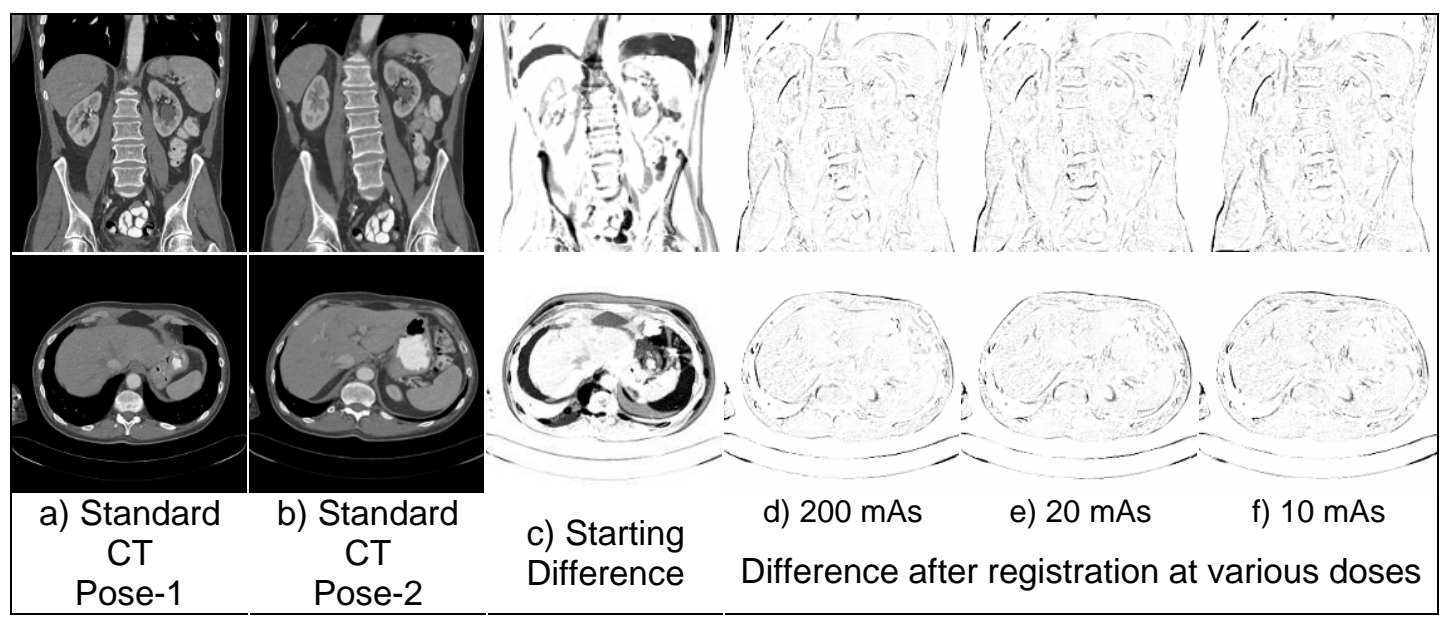

Figure 6.5: Qualitative comparison of registration accuracy with low-dose CT.

reduced features in the difference image) demonstrates the feasibility of deformable registration at low CT doses.

The process of deformable registration attempts to recover any misalignment between the reference and floating images. A perfect registration will completely recover this misalignment and yield a non-rigid transformation field that is identical to the deformation field representing the original misalignment. A comparison between these two fields can be used as a performance index for the registration accuracy.

For this experiment, the deformation field introduced $\left(\mathrm{DF}_{\mathrm{i}}\right)$ is known at every voxel. The volume subdivision-based deformable registration algorithm generates the transformation field $\left(\mathrm{RF}_{\mathrm{j}}\right)$, which provides the transformation at every voxel in scan with dose $\mathrm{j}$. The average of the magnitude of the vector differences between these two fields at all doses for the software implementation is reported in Figure 6.6. This average was calculated over the region of the image which contains sufficient part of the subject and hence information to yield meaningful registration. The regions of the image which contain no information (very low entropy) (e.g. black areas surrounding 
the subject) are masked out using a simple threshold operation. The results show a maximum error of about $10 \%$ at the doses of $10 \mathrm{mAs}$ and $20 \mathrm{mAs}$, respectively. Moreover the maximum registration error at the lowest dose (10 mAs) is less than 2.5 mm, which is within acceptable limits of IGI applications. As expected, the average error improves steadily with dose.

We repeated the experiment with the high-speed implementation of the deformable registration algorithm described earlier, and compared the registration field produced by the hardware implementation with the user induced deformation. The same optimization algorithm (downhill Simplex) was used for these two implementations and equal number of optimization iterations were used. Figure 6.6 also shows the comparison of the average registration accuracy achieved using the software and hardware implementations. The blue curve corresponds to the average registration accuracy using the software implementation and the red curve corresponds to the average registration accuracy of the hardware implementation.

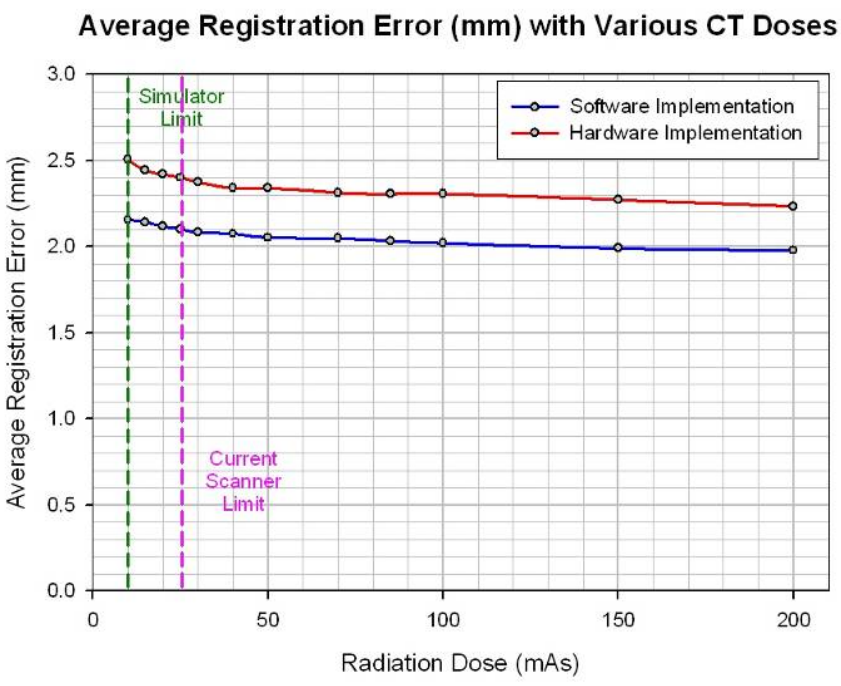

Figure 6.6: Average registration error with respect to dose using software and FPGA-based implementations. 
Table 6.1: $\quad$ Execution time for deformable image registration using low-dose CT. Execution Time (s)

\begin{tabular}{ccc}
\hline $\begin{array}{c}\text { Software } \\
\text { Implementation }\end{array}$ & $\begin{array}{c}\text { FPGA-based } \\
\text { Implementation }\end{array}$ & Speedup \\
\hline 11,976 & 392 & 30.55 \\
\hline
\end{tabular}

This comparison of the registration accuracies indicates that the hardware implementation is capable of achieving comparable registration accuracy. The average execution time for these two implementations is reported in Table 6.1. The hardware implementation achieved a speedup of about 30 and offers comparable registration accuracy. Moreover, both the implementations offer acceptable registration accuracy for most IGI applications and demonstrate the applicability of deformable image registration to lower the radiation dose during intraprocedural imaging.

\subsubsection{Summary}

In this study, we have demonstrated successful registration of standard-dose abdominal CT images with lower-dose images of the same anatomy. Even at $10 \mathrm{mAs}$, the smallest dose achievable using the simulator, the registration accuracy achieved was comparable to that achieved at the standard dose. Our results demonstrate ten- to twenty fold reduction in radiation dose with the use of low-dose CT. Reduction of radiation dose to safe levels is highly significant in that it enables navigating interventions using more powerful, multislice CT. Moreover, through the use of hardware accelerated implementation, deformable image registration (which allows this reduction in radiation dose) can be performed in a matter of minutes. This enables assimilation of this novel dose reduction strategy in the IGI workflow. The 
introduction of true 3D visualization possible through safe, volumetric CT imaging of the intraprocedural anatomy may enable development of novel image-guided interventional applications such as CT-guided minimally invasive surgery [149, 184].

\subsection{Incorporation of PET into CT-Guided Liver Radio-Frequency}

\section{Ablation}

I would like to thank Mr. Peng Lei from Prof. Shekhar's research group for his help in the quantitative validation aspect of the deformable registration presented in this section.

\subsubsection{Motivation}

The liver is a common site of both primary and metastatic cancers. Performing resection of malignant liver tumors has been shown to increase the 5-year survival rate of patients with liver cancer [185]. However, the majority (85\%-90\%) of hepatic tumors are considered unresectable at diagnosis [186], either because of their anatomic location, size, or number or because of inadequate viable liver tissue and morbidity. Radiofrequency ablation (RFA) as a minimally invasive procedure is often the treatment of choice for patients who are not suitable candidates for resection [187]. RFA is a treatment technique that uses high-frequency alternating electrical current to destroy tissue cells by heating [188]. For small lesions ( $<5 \mathrm{~cm}$ in diameter), RFA has been reported to achieve 4-year survival rates, comparable with those achieved with resection $[189,190]$.

RFA has conventionally been performed under fluoroscopic or ultrasound guidance. With the advent of multislie CT, many of these procedures are being 
carried out under intraprocedural volumetric CT guidance. The volumetric CT scan provides better 3D orientation and a detailed anatomic structural map. However, some lesions (particularly small untreated masses or recurrent or residual tumors in a large treated mass) are not clearly visible (see Figure 2.1) because intraprocedural CT scanning is usually not contrast enhanced. Even in contrast-enhanced diagnostic CT images, hepatic lesions with abnormal metabolic activity are sometimes overlooked [191]. Consequently, local recurrence after liver RFA as a result of these missed tumors remains one of the major factors in relapse [192], which is in the range of 3\%-39\% [193]. Thus, precise targeting of lesions remains a challenging task when guided solely by volumetric CT with or without contrast enhancement.

Because malignancies are better characterized by increased metabolic activity than CT number variations, PET (as a functional imaging modality) has higher sensitivity and specificity than CT for tumor localization. Active lesions show up clearly as regions of high uptake in a conventional PET scan. However, compared with CT, PET is devoid of structural tissue details, which are also important for intraprocedural targeting and needle placement. Moreover, PET is also challenged by slow scanning speed, radiation exposure risks, logistic challenges, etc. Currently PET remains primarily a preprocedural imaging modality, used to identify a treatment site rather than provide intraprocedural guidance during an ablative procedure. To combine the strengths and overcome disadvantages of both PET and CT as intraprocedural imaging modalities, registration between PET and CT is essential. In a clinical study by Veit et al. [186], PET and CT fusion images were reported to greatly improve recognition and localization of liver masses. Our work, therefore, is 
focused on demonstrating fast, automatic, and accurate deformable image registration between preprocedural PET and intraprocedural CT to improve localization of liver malignancies during RFA.

\subsubsection{Registration of PET and CT}

As described earlier, combining of PET and CT images is crucial during liver RFA. PET, however, cannot be repeated intraprocedurally because of time and logistic challenges, as well as radiation risks [194]. Consequently, the interventionist must mentally correlate preprocedural PET data onto intraprocedural CT images to localize the lesion into which the RFA needle will be advanced, a subjective task dependent on operator expertise. Combined PET/CT scanners, which are based on mechanically achieved rigid registration, have emerged in recent years. They have provided significant improvement over separate CT and PET scanning. But in abdominal procedures such as liver RFA (unlike thoracic or brain surgery), non-rigid misalignment resulting from tissue deformation and respiration motion can be significant, so rigid registration approaches such as combined PET/CT scanning and fiducial marker-based registration may misrepresent the actual transformation of the liver. Combined PET/CT scanners have been shown to be unable to fully compensate for involuntary non-rigid motions, such as those from respiration [185], and the resulting images have been found to have significant misregistration in areas close to the diaphragm. Moreover, as mentioned previously, PET has challenges including slow scanning speed and radiation exposure risks. A combined PET/CT scanner is not an appropriate choice as an intraprocedural imaging modality in this application or any other image-guided procedure. 
Intensity-based, retrospective deformable image registration and fusion together form the only alternative, which is also proposed here. To be clinically practical, the registration algorithm must be automatic, which means that manual segmentation-based registration is excluded. Moreover, as mentioned before, the registration must be achieved sufficiently fast so as not to obstruct the clinical workflow. The deformable registration algorithm described in the dissertation has been previously validated for intensity based registration of whole-body PET and CT images [66] and PET/CT images for liver RFA [78, 148]. In this work, we validate this algorithm and its FPGA-based high-speed implementation of this algorithm in the context of the aforementioned clinical application.

\subsubsection{Experiments}

This study was geared toward demonstrating the feasibility of image registration between preprocedural PET and intraprocedural CT in the context of liver RFA using the earlier described high-speed image registration solution. This retrospective study involved 20 CT-PET image pairs of patients who had undergone percutaneous abdominal RFA under CT guidance. The preprocedural PET scans had size and resolution of $150 \times 150 \times 187-240$ and $4.0 \times 4.0 \times 4.0 \mathrm{~mm}$, respectively. Noncontrast-enhanced intraprocedural abdominal CT scans were acquired for guiding needle placement during RFA procedures. The intraprocedural CT scans had size and resolution of $512 \times 512 \times 35-62$ and $0.78-1.17 \times 0.78-1.17 \times 5 \mathrm{~mm}$, respectively. 


\subsubsection{Image Preprocessing}

The intraprocedural CT images were acquired during the RFA with the RFA needle in the field of view. As a result, some metal artifacts were present in the CT images. We preprocessed the CT images using 3D median filtering and 3D anisotropic diffusion filtering in order to minimize these artifacts. To achieve a trade-

off between maintaining CT resolution and obtaining nearly isotropic voxels, we resampled the intraprocedural CT images for all cases to have the dimensions of 256 $\times 256 \times 128$. Resampling reduced the spatial resolution of CT images in $x$ and $y$ dimensions; however, the resulting images still had better spatial resolution than the preprocedural PET images (the lower resolution image controls the accuracy of intensity-based image registration in general). No preprocessing steps were used for the preprocedural PET images.

\subsubsection{Validation of Image Registration}

We evaluated the accuracy of the deformable registration between PET and CT by comparing the alignment of several anatomic landmarks (3D locations within the images) as predicted by the algorithm (both software and FPGA-based implementations) against a reference. Because of the lack of a gold standard for validation of deformable registration algorithms, we assumed the ability of clinical experts to locate landmarks in both intraprocedural CT and preprocedural PET images as a suitable benchmark performance. We then contend that comparing the variability in landmark matching between algorithm- and expert-defined registrations with the variability among the three expert-defined correspondence between the 


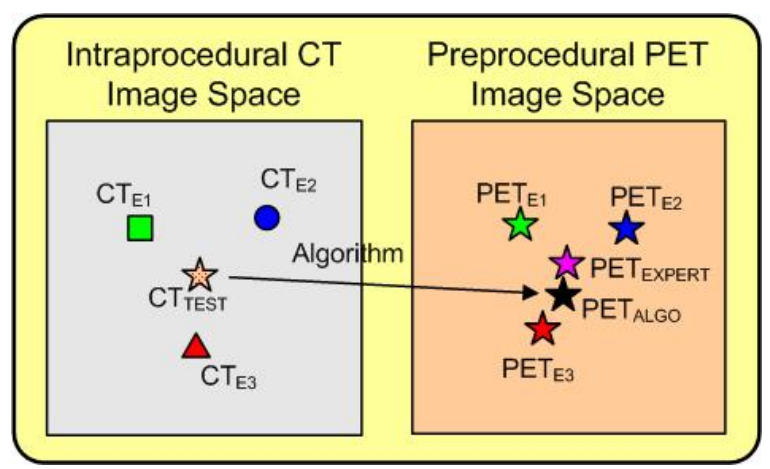

Figure 6.7: Graphic illustration of the quantitative validation approach used in the context of deformable registration between intraprocedural and preprocedural PET.

landmarks is a reasonable way to evaluate the registration accuracy of the algorithm and assess whether its performance is comparable to that of the experts.

Our validation scheme is graphically represented in Figure 6.7. Three clinical experts, experienced in interpreting CT and PET images, were involved in the validation procedure. Each expert was asked to identify and mark anatomic landmarks identifiable in both PET and CT images. Because the location of a specific landmark as marked by an expert can vary slightly from expert to expert, a set of "test landmarks” were created for each case separately. This was achieved by defining the location of each landmark as the centroid of the expert-defined locations for that landmark in intraprocedural CT (represented by $\mathrm{CT}_{\mathrm{TEST}}$ in Figure 6.7). The expertdefined transformation fields were then used to determine distinct sets of homologous preprocedural PET landmarks ( $\mathrm{PET}_{\mathrm{E} 1}, \mathrm{PET}_{\mathrm{E} 2}$, and $\mathrm{PET}_{\mathrm{E} 3}$, respectively), each representing the transformed locations of the test landmark $\left(\mathrm{CT}_{\mathrm{TEST}}\right)$ according to the manual registration performed by each expert. The average expert-defined transformed location (PET $\mathrm{PXPERT}_{\text {) }}$ was calculated as the centroid of $\mathrm{PET}_{\mathrm{E} 1}, \mathrm{PET}_{\mathrm{E} 2}$, and $\mathrm{PET}_{\mathrm{E} 3}$. The algorithm-determined transformation field was used to determine a 
set of landmarks in the PET image ( $\mathrm{PET}_{\mathrm{ALGO}}$ ) representing the transformed locations of the test landmarks after automatic deformable registration. These locations were calculated for both the software and FPGA-based high-speed implementation of the algorithm.

For each case, the mean error between PET $_{\text {EXPERT }}$ and $\mathrm{PET}_{\mathrm{ALGO}}$ was then evaluated to quantify the registration accuracy for that case. To further evaluate the algorithm performance in the context of inter-expert variability, we allocated the 4 sets of PET landmark points to separate groups of 3 sets each: reference group ( $\mathrm{PET}_{\mathrm{E} 1}, \mathrm{PET}_{\mathrm{E} 2}$, and $\mathrm{PET}_{\mathrm{E} 3}$ ); test group $1\left(\mathrm{PET}_{\mathrm{ALGO}}, \mathrm{PET}_{\mathrm{E} 2}\right.$, and $\left.\mathrm{PET}_{\mathrm{E} 3}\right)$; test group 2 ( $\mathrm{PET}_{\mathrm{E} 1}, \mathrm{PET}_{\mathrm{ALGO}}$, and $\left.\mathrm{PET}_{\mathrm{E} 3}\right)$; and test group 3 ( $\mathrm{PET}_{\mathrm{E} 1}, \mathrm{PET}_{\mathrm{E} 2}$, and $\left.\mathrm{PET}_{\mathrm{ALGO}}\right)$. For each group, the mean difference (Euclidean distance) in the transformed location of corresponding landmarks was obtained for all pair wise combinations of sets of PET points within that group. The mean difference for each group was determined by averaging over all the cases. The variability of locations was then calculated for each

group. If the group variability of test groups 1,2 , and 3 is statistically similar to that of the reference group, we can conclude that the algorithm and experts agree on the PET location of a specific landmark in CT. If the group variability is statistically different, the algorithm differs significantly from that of the experts. As mentioned earlier, we performed this analysis for both software and the FPGA-accelerated implementation of the deformable registration algorithm.

\subsubsection{Results}

All the cases were successfully registered when evaluated both qualitatively and quantitatively. Both the software and hardware implementations yielded 
qualitatively similar results. The average execution time for these two implementations is reported in Table 6.2. The hardware implementation provides a speedup of around 30 and offers comparable registration accuracy, as presented later. Qualitative evaluation included visual assessment of improvement in image alignment by the clinical experts. No visually apparent gross misregistration was found in any case. Example of registration using FPGA-based high-speed implementation of the intensity-based deformable registration is presented in Figure 6.8. For this example, axial, coronal, and sagittal views through the intraprocedural CT and preprocedural PET, as well as fused PET-CT images before and after deformable image registration, are shown. The first row shows the original preprocedural CT image. The second row shows the unregistered preprocedural PET image. The third row presents the fusion of intraprocedural CT and preprocedural PET prior to image registration. Misalignment of anatomical structures in this fusion image is indicated by overlaid arrows. The final row illustrates the fusion of intraprocedural CT with registered (through FPGA-based deformable image registration) PET image. In all three views, deformable image registration between PET and CT images provided superior image alignment when compared with unregistered fusion images. Deformable registration recovered the misalignment between anatomical structures and lesions. For example, in Figure 6.8, edges of anatomical structures in PET and CT agree quite well. 
(a)

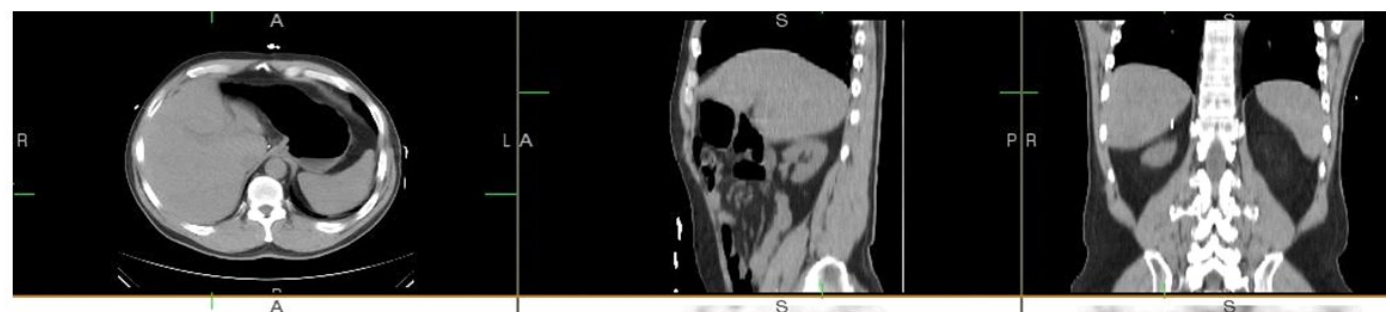

(c)

(b) ${ }^{\text {P }}$
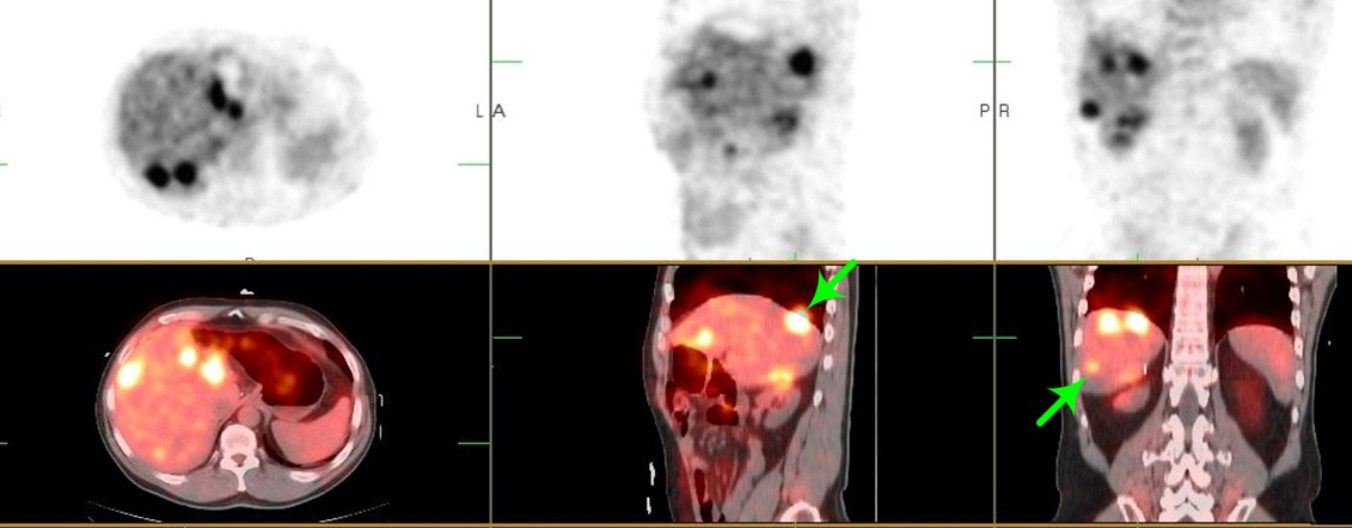

(d)

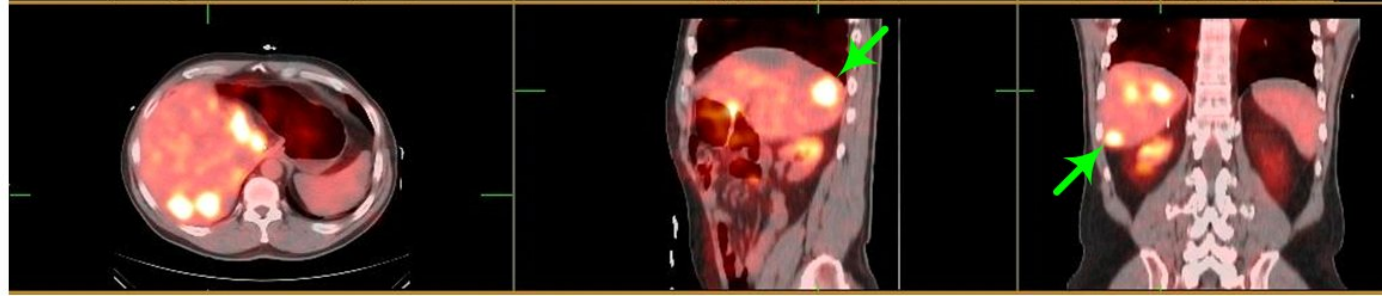

Figure 6.8: $\quad$ Registration of intraprocedural CT and preprocedural PET images using the FPGA-based implementation of deformable image registration.

We performed the quantitative validation of deformable registration using the validation strategy described earlier. Table 6.3 presents the results of this validation for software implementation. The interexpert variability (i.e., group variability) in the identification of each of the four landmarks was averaged for all the 20 image pairs after software-based registration between intraprocedural CT and preprocedural PET.

Table 6.2: $\quad$ Execution time for deformable image registration using intraprocedural CT and preprocedural PET images.

\begin{tabular}{ccc}
\hline \hline \multicolumn{2}{c}{ Execution Time (s) } & Speedup \\
$\begin{array}{ccc}\text { Software } \\
\text { Implementation }\end{array}$ & $\begin{array}{c}\text { FPGA-based } \\
\text { Implementation }\end{array}$ & \\
\hline \hline 5217 & 164 & 31.81 \\
\hline
\end{tabular}


The t-tests showed no statistically significant difference between the reference group and any test group for any of the four landmarks (the reference group value lies within the 95\% confidence intervals of all test groups), indicating that the algorithm’s solutions were statistically similar to those of experts. For quantitative validation of the FPGA-based implementation, we repeated the above validation procedure, replacing the software implementation by the FPGA-based implementation. Table 6.4 presents the results of this quantitative validation. The TRE derived from the mean of $\left|\mathrm{PET}_{\text {EXPERT }}-\mathrm{PET}_{\mathrm{ALGO}}\right|$ over all cases and landmarks was $6.7 \mathrm{~mm}$ and $7.0 \mathrm{~mm}$ for software and hardware-accelerated implementations, respectively. This result is comparable with the accuracy reported earlier for deformable registration using whole-body 3D PET-CT images [66]. This indicates that the accuracy of the deformable image registration algorithm is approximately independent of the anatomy.

The TRE obtained for the FPGA-based, high speed implementation of

Table 6.3: Interexpert variability in landmark identification across 20 image pairs. PET $_{\mathrm{ALGO}}$ corresponds to the software implementation of the algorithm.

\begin{tabular}{|c|c|c|c|}
\hline \multicolumn{4}{|c|}{$\begin{array}{c}\text { Mean Difference in Landmark Location (mm) ( 95\% Confidence } \\
\text { Interval (mm) ) } \\
P \text { Value }\end{array}$} \\
\hline $\begin{array}{l}\text { Reference group } \\
\left(\mathrm{PET}_{1}, \mathrm{PET}_{2} \text {, }\right. \\
\left.\mathrm{PET}_{3}\right)\end{array}$ & $\begin{array}{c}\text { Group } 1 \\
\left(\mathrm{PET}_{\mathrm{ALGO}}, \mathrm{PET}_{2} \text {, }\right. \\
\left.\mathrm{PET}_{3}\right)\end{array}$ & $\begin{array}{c}\text { Group } 2 \\
\left(\mathrm{PET}_{1}, \mathrm{PET}_{\mathrm{ALGO}}\right. \\
\left.\mathrm{PET}_{3}\right)\end{array}$ & $\begin{array}{c}\text { Group 3 } \\
\left(\mathrm{PET}_{1}, \mathrm{PET}_{2} \text {, }\right. \\
\left.\text { PET }_{\mathrm{ALGO}}\right)\end{array}$ \\
\hline 6.7 & $\begin{array}{c}6.8(6.2,7.4) \\
P=0.76\end{array}$ & $\begin{array}{c}7.2(6.6,7.7) \\
P=0.11\end{array}$ & $\begin{array}{c}7.0(6.4,7.6) \\
P=0.34\end{array}$ \\
\hline 6.5 & $\begin{array}{c}6.4(5.9,6.9) \\
P=0.76\end{array}$ & $\begin{array}{c}6.2(5.7,6.7) \\
P=0.22\end{array}$ & $\begin{array}{c}6.5(6.0,7.0) \\
P=0.95\end{array}$ \\
\hline 6.1 & $\begin{array}{c}6.0(5.4,6.6) \\
P=0.74\end{array}$ & $\begin{array}{c}6.2(5.5,6.8) \\
P=0.76\end{array}$ & $\begin{array}{c}6.3(5.7,7.0) \\
P=0.52\end{array}$ \\
\hline 5.8 & $\begin{array}{c}5.6(5.1,6.1) \\
P=0.43\end{array}$ & $\begin{array}{c}5.7(5.2,6.2) \\
P=0.64\end{array}$ & $\begin{array}{c}6.0(5.5,6.5) \\
P=0.39\end{array}$ \\
\hline
\end{tabular}


Table 6.4: Interexpert variability in landmark identification across 20 image

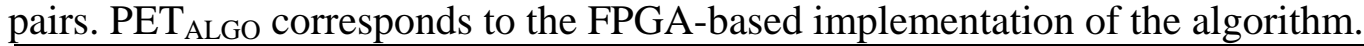

\section{Mean Difference in Landmark Location (mm) ( 95\% Confidence Interval (mm))}

$P$ Value

\begin{tabular}{|c|c|c|c|c|}
\hline Group & $\begin{array}{c}\text { Reference group } \\
\left(\mathrm{PET}_{1}, \mathrm{PET}_{2},\right. \\
\left.\mathrm{PET}_{3}\right) \\
\end{array}$ & $\begin{array}{c}\text { Group } 1 \\
\left(\mathrm{PET}_{\mathrm{ALGO}, \mathrm{PET}_{2},}\right. \\
\left.\mathrm{PET}_{3}\right) \\
\end{array}$ & $\begin{array}{c}\text { Group } 2 \\
\left(\mathrm{PET}_{1}, \mathrm{PET}_{\mathrm{ALGO}}\right. \\
\left.\mathrm{PET}_{3}\right) \\
\end{array}$ & $\begin{array}{c}\text { Group } 3 \\
\left(\mathrm{PET}_{1}, \mathrm{PET}_{2} \text {, }\right. \\
\left.\mathrm{PET}_{\mathrm{ALGO}}\right) \\
\end{array}$ \\
\hline $\begin{array}{l}\text { Dome } \\
\text { of Liver }\end{array}$ & 6.7 & $\begin{array}{c}7.1(6.5,7.8) \\
P=0.16\end{array}$ & $\begin{array}{c}7.3(6.6,8.0) \\
P=0.08\end{array}$ & $\begin{array}{c}7.2(6.5,7.9) \\
P=0.13\end{array}$ \\
\hline $\begin{array}{l}\text { Inferior Tip } \\
\text { of Liver }\end{array}$ & 6.5 & $\begin{array}{c}6.8(6.3,7.4) \\
P=0.32\end{array}$ & $\begin{array}{c}6.5(5.9,7.0) \\
P=0.88\end{array}$ & $\begin{array}{c}6.8(6.2,7.4) \\
P=0.38\end{array}$ \\
\hline $\begin{array}{l}\text { Right Kidney } \\
\text { Upper Pole }\end{array}$ & 6.1 & $\begin{array}{c}6.3(5.6,7.0) \\
P=0.50\end{array}$ & $\begin{array}{c}6.5(5.7,7.2) \\
P=0.32\end{array}$ & $\begin{array}{c}6.4(5.7,7.1) \\
P=0.36\end{array}$ \\
\hline $\begin{array}{l}\text { Right Kidney } \\
\text { Lower Pole }\end{array}$ & 5.8 & $\begin{array}{c}5.7(5.2,6.2) \\
P=0.70\end{array}$ & $\begin{array}{c}5.9(5.4,6.4) \\
P=0.67\end{array}$ & $\begin{array}{c}6.2(5.8,6.7) \\
P=0.06\end{array}$ \\
\hline
\end{tabular}

deformable image registration is slightly inferior to that obtained using the software implementation. However, the t-tests indicate no statistically significant difference between the reference group and any test group for any of the four landmarks (the reference group value lies within the $95 \%$ confidence intervals of all test groups). This further indicates that the registration solutions provided by the FPGA-based implementation were statistically similar to those of experts. Thus, the hardware solution developed in this dissertation not only reduces the execution time of deformable registration to a few minutes, but also offers accuracy that is statistically similar to that of a group of clinical experts. This fast and accurate implementation can, therefore, be used to incorporate preprocedural PET images during CT-guided liver RFA.

\subsubsection{Summary}

In this study, we have demonstrated successful registration of intraprocedural abdominal CT images acquired during liver RFA with preprocedural PET images. 
This can enable incorporation of PET into RFA procedures through algorithmic image registration. The hardware-accelerated implementation of image registration, developed as part of this dissertation work, can perform this image registration task in a matter of minutes and yet offers comparable accuracy. This represents a first significant step toward integration deformable registration in RFA procedures. With further technological advances, such as integration with the imaging equipment, this approach can be made routine under clinical settings. This would lead to precise ablation of the area of malignant activity, as indicated on PET, and avoid unnecessary ablation of healthy tissues. For large and multiple lesions, this technique may shorten procedure time and minimize post-procedural morbidity. Precise targeting of lesions could allow definitive and complete treatment, potentially reducing relapse rates and the number of repeat procedures. 


\section{Chapter 7: Conclusions and Future Work}

\subsection{Conclusion}

Minimally invasive IGIs are time and cost efficient, minimize unintended damage to healthy tissue, and lead to faster patient recovery. Consequently, these procedures are becoming increasingly popular. With the availability of high-speed volumetric imaging devices there is an increasing thrust on using $3 \mathrm{D}$ images for navigation and target delineation during IGIs. However, processing and analysis of these volumetric images, while meeting the on-demand performance requirements of IGI applications remains a challenging task. To address this problem, this dissertation has presented core components of an advanced image-guidance system that will allow high-speed processing and analysis of these images. The execution performance along with the accuracy and compact and reconfigurable nature of these components enables their integration into clinical applications.

Image preprocessing and enhancement is an important prerequisite step in the IGI workflow prior to advanced image analysis and visualization. Chapter 3 presented a novel FPGA-based architecture for real-time preprocessing of volumetric images acquired during IGIs. We presented a brick-caching scheme that allows efficient, low-latency access to sequential 3D neighborhoods, a scenario common to most filtering operations. We introduced an FPGA-based implementation of 3D anisotropic diffusion filtering. This design takes advantage of the symmetries present in the Gaussian kernel and implements this filtering kernel with a reduced number of multipliers. We further presented a linear systolic array-based architecture for 
accelerated implementation of 3D median filtering. The developed architecture enables 3D anisotropic diffusion filtering and 3D median filtering of intraprocedural images at the rate of $50 \mathrm{fps}$, which is faster than current acquisition speeds of most intraprocedural imaging modalities. The solution presented offers real-time performance, is compact and accurate, and, hence, suitable for integration into IGI workflow. Furthermore, the additional filtering kernels that are based on neighborhood operations (for example, general purpose convolution) can be easily incorporated into the same framework.

As IGI applications become increasingly popular, intraprocedural imaging modalities continue to offer wider coverage and higher imaging speed. Thus, there is a corresponding need for real-time processing of these images. The real-time performance of our design along with the throughput of one voxel per cycle can cater to these $4 \mathrm{D}(3 \mathrm{D}+$ time $)$ image processing needs.

Image registration between intra- and preprocedural images is a fundamental need in the IGI workflow. To facilitate that, Chapter 4 presented a novel FPGA-based architecture for high-speed implementation of MI-based deformable image registration. This architecture achieved voxel-level parallelism through pipelined implementation and employed several strategies to address the fundamental bottleneck in the intensity-based image registration, namely memory access management. As a result of these enhancements, the presented architecture is capable of achieving high voxel processing rate and a speedup of about 30 and consequently reduces the execution time of deformable registration from hours to only a few minutes. The results of the qualitative and quantitative validation demonstrate that 
this performance improvement does not significantly compromise the accuracy of deformable registration. Further clinical validation performed in the context of novel IGI applications illustrated the potential of this implementation to enable improved target delineation during image-guided interventions through deformable registration with preprocedural images. The robustness, speed, and accuracy offered by this architecture, in conjunction with its compact implementation, make it ideally suitable for integration into IGI workflow.

Accurate, robust, and real-time deformable image registration between intraand preprocedural images has been an unmet need, critical to the success of imageguided procedures. The work presented in this dissertation constitutes an important first step toward meeting this goal. With further algorithmic and hardware improvements, geared toward enhancing its accuracy and performance, this approach has the potential to elevate the precision of current procedures and expand the scope of IGI to moving and deformable organs.

The work presented in this dissertation achieved superior performance through custom design on a reconfigurable computing platform, by addressing the fundamental bottlenecks in the considered computationally intensive applications. One of the primary strengths of reconfigurable architectures over general purpose processor-based implementations is their ability to utilize more streamlined representations for internal variables. Furthermore, this approach can result into optimized utilization of FPGA resources, by employing just enough precision for each of its internal variables to satisfy design requirements of a given application. Given this advantage, it is highly desirable to automate the derivation of optimized 
design configurations that offer varying degree of tradeoff between implementation accuracy and hardware resources. Toward that end, this dissertation has presented a framework for multiobjective optimization of finite precision, reconfigurable implementations. This framework considered multiple conflicting objectives, such as hardware resource consumption and implementation accuracy, and systematically explored tradeoff relationships among the targeted objectives. This dissertation has also further demonstrated the applicability of EA-based techniques for efficiently identifying Pareto-optimized tradeoff relations in the presence of complex and nonlinear objective functions. The evaluation that this work has conducted in the context of the FPGA-based architecture for deformable image registration demonstrated that such an analysis can be used to enhance automated hardware design processes, and to efficiently identify a system configuration that meets given design constraints. This approach may also be applied in the context of reconfigurable computing for identifying suitable design configurations that can be switched among at runtime. Furthermore, the multiobjective optimization approach presented in this dissertation is quite general, and can be extended to a multitude of other signal processing applications.

We have extensively validated the applicability of our approach in the context of CT-guided interventions. In the first clinical application, we demonstrated successful registration of standard-dose abdominal CT images with lower-dose images of the same anatomy. Our results demonstrated ten- to twenty fold reduction in intraprocedural radiation dose through the use of low-dose CT. Reduction of radiation dose to safe levels is highly significant in that it enables navigating 
interventions using more powerful, multislice CT. Moreover, through the use of hardware accelerated implementation, deformable image registration (which allows this reduction in radiation dose) can be performed in a matter of minutes. This enables assimilation of this novel dose reduction strategy in the IGI workflow. In the second IGI application, we have further demonstrated successful registration of intraprocedural abdominal CT images acquired during liver RFA with preprocedural PET images. This can enable incorporation of PET into RFA procedures through algorithmic image registration. We demonstrated that the hardware-accelerated implementation of image registration, developed in this dissertation work, can perform this image registration task in a matter of minutes and yet offers comparable accuracy.

Our approach represents a first significant step toward integration of 3D image processing and deformable image registration in image-guided procedures. This approach can not only improve target delineation and reduce radiation dose but can also trigger the development of novel image-guided procedures. With further technological advances, such as integration with the imaging and visualization equipment, and additional enhancements in speed and accuracy, this approach can be made routine under clinical settings. This approach has the potential to elevate the precision of current IGI procedures, expand the scope of IGI to moving and deformable organs, and thereby to provide a new level of sophistication and accuracy during IGIs. 


\subsection{Future Work}

The FPGA-based architecture for real-time implementation of image preprocessing operations is capable of providing a high voxel throughput and a volumetric processing rate higher than the acquisition speed of most current generation imaging modalities. This architecture presented implementation of 3D anisotropic diffusion filtering and 3D median filtering, the preprocessing steps most commonly used in the context of IGI. However, the core components of this architecture, in particular the memory controller and the brick-caching scheme, are general enough to allow real-time realization of a range of image processing kernels based on neighborhood operations. For example, a kernel for general-purpose 3D convolution reported by Venugopal et al. [195] can easily be incorporated in the same image processing framework. Similarly, certain morphological and contrastenhancement operations that are based on neighborhood operations can also be supported by the same framework while providing equivalent voxel throughput. The current implementation of this framework supports sequential image processing operations through static reconfiguration of the filtering kernels. With the advent of modern FPGAs that are capable of runtime reconfiguration, it is possible to support adaptive image preprocessing by changing the preprocessing steps or tuning the filtering kernel parameters at run-time. This will enable the preprocessing system to adapt to the requirements of the subsequent advanced image processing applications (for example, registration, volume rendering, or segmentation).

The architecture presented for accelerated calculation of MI, is capable of achieving deformable registration between a pair of images with size $256 \times 256 \times 256$ 
in about 6 minutes, while providing accuracy comparable to a software implementation. This represents a significant first step toward enabling integration of deformable registration in the IGI workflow. Further acceleration of the aforementioned registration algorithm to satisfy the interactive requirement of IGIs can be achieved through additional strategies. First, the current architecture uses the same external memory module to store both the RI and FI. Storing these images in two separate memory modules will allow their independent parallel access. This will eliminate the need to prefetch the RI voxels and thus provide speedup. In addition, using high-speed static random access memory (SRAM) modules for storing the randomly accessed FI is likely to provide further speedup by providing faster access to the FI with minimal latencies. Second, as showed by Studholme et al. [64], varying MH size between $32 \times 32$ and $256 \times 256$ does not significantly affect the accuracy of MI-based registration. Based on this observation, the size of the $\mathrm{MH}$ within the FPGA-based implementation can be adaptively reduced with every level of subdivision. This will reduce the overhead of clearing the $\mathrm{MH}$ for smaller subvolumes, thereby lending additional speedup. Third, the overhead of communication time required for exchanging the transformation matrix and the calculated MI value between the host and the MI calculator can be minimized by reducing the communication latency. This will provide additional speedup, especially at finer levels of image subdivision where the computation time becomes comparable to the communication time. Most modern FPGAs support an embedded hard- or softprocessor core that can be utilized to implement the optimization algorithm. Thus, both the components of the registration routine will be located on the same platform, 
thereby reducing the communication latency. Finally, as described earlier, the registration algorithm optimizes the individual subvolumes at a given level of subdivision sequentially, but independently of each other. Thus, using multiple FPGA modules in parallel it is possible to simultaneously optimize these subvolumes. This multi-FPGA implementation will likely provide near-linear speedup. This architecture can also be incorporated in a broader, heterogeneous computing framework such as the one described by Plishker et al. [121]. All these strategies, in combination, can further reduce the execution time of deformable image registration and ultimately achieve near-real time performance for its seamless integration into IGI applications.

The framework we presented for optimization of finite-precision implementations considers multiple conflicting objectives, such as hardware resource consumption and implementation accuracy, and systematically explores tradeoff relationships among the targeted objectives. Although this framework was developed and validated in the context of FPGA-based image registration, the presented optimization approach is quite general and can be extended to many signal processing applications beyond medical image processing domain. However, for demonstration of this capability, further validation of this optimization strategy must be performed in the context of multiple signal processing applications with known benchmarks. The hardware area models we adopted while developing this framework, model the FPGA area consumption using the number of look-up tables required. Modern FPGA families, however, provide a large number of special functional units that may be utilized for performing arithmetic operations such as multiplication, addition, etc. 
Although we demonstrated the high fidelity of the adopted area models, enhancing these models to take into account the utilization of specialized functional units will better represent the area requirements of a design configuration and, in general, provide more accurate area estimation. In addition to the approaches mentioned above, the framework for optimization of finite precision implementations can be enhanced in the following ways: first, the search methods could be refined further to efficiently explore the search space. For example, the multi-objective optimization could be preceded by univariable simulations that can help to reduce the size of the search space. Also, the parameters used for EA-based search, such as representation scheme, population size, crossover and mutation operators, etc. can be optimized as well. Second, the framework could be tuned for automated selection of search methods that are ideally suited for a given problem. For example, for small design search spaces a technique based on exhaustive search could be utilized. Further, EAbased search schemes could be enhanced further by exploring selection schemes based on techniques such as NSGA-II, epsilon dominance and quality indicators. Finally, this framework and/or the Pareto-optimized solutions identified by this framework can be incorporated into automated design optimization flows. This will enable selection of optimized design configurations that balance the tradeoff between implementation accuracy and hardware cost at run-time. Further, additional objective functions such as power requirement and operating frequency could also be incorporated in this framework to comprehensively capture the effects of various design configurations. 


\section{Bibliography}

[1] W. Charboneau, "Image-guided Therapy is New Pillar in Patient Care," Radiology Society of North America, New Horizons Lecture, 2006.

[2] C. Fujioka, J. Horiguchi, M. Ishifuro, H. Kakizawa, M. Kiguchi, N. Matsuura, M. Hieda, T. Tachikake, F. Alam, T. Furukawa, and K. Ito, "A feasibility study: Evaluation of radiofrequency ablation therapy to hepatocellular carcinoma using image registration of preoperative and postoperative CT," Academic Radiology, vol. 13(8), pp. 986-994, Aug 2006.

[3] D. E. Heron, R. S. Andrade, and R. P. Smith, "Advances in image-guided radiation therapy--the role of PET-CT," Medical Dosimetry, vol. 31(1), p. 3, 2006.

[4] P. Veit, C. Kuehle, T. Beyer, H. Kuehl, A. Bockisch, and G. Antoch, "Accuracy of combined PET/CT in image-guided interventions of liver lesions: an ex-vivo study," World journal of gastroenterology, vol. 12(15), pp. 2388-2393, 2006.

[5] V. Vilgrain, "Tumour detection in the liver: role of multidetector-row CT," European radiology, p. D85, 2005.

[6] J. T. Yap, J. P. J. Carney, N. C. Hall, and D. W. Townsend, "Image-guided cancer therapy using PET/CT," Cancer journal, vol. 10(4), pp. 221-233, 2004.

[7] K. Benkrid, D. Crookes, and A. Benkrid, "Design and implementation of a novel algorithm for general purpose median filtering on FPGAs," Proc. of the IEEE International Symposium on Circuits and Systems, ISCAS, vol. 4(pp. 425-428, 2002.

[8] T. Gijbels, P. Six, L. Van Gool, F. Catthoor, H. De Man, A. Oosterlinck, J. Rabaey, P. M. Chau, and J. Eldon, "A VLSI-architecture for parallel non-linear diffusion with applications in vision," Proc. IEEE Workshop on VLSI Signal Processing, pp. 398-407, 1994.

[9] C. L. Lee and C. W. Jen, "A bit-level scalable median filter using simple majority circuit," Proc. of IEEE International Symposium on VLSI Technology, Systems and Applications, pp. 174-177, 1989.

[10] M. Rumpf and R. Strzodka, "Nonlinear Diffusion in Graphics Hardware," Proceedings of IEEE TCVG Symposium on Visualization, pp. 75-84, 2001.

[11] K. Wiehler, J. Heers, C. Schnorr, H. S. Stiehl, and R. R. Grigat, "A onedimensional analog VLSI implementation for nonlinear real-time signal preprocessing," Real-Time Imaging, vol. 7(1), pp. 127-142, 2001.

[12] C. Kremser, C. Plangger, R. Bosecke, A. Pallua, F. Aichner, and S. R. Felber, "Image registration of MR and CT images using a frameless fiducial marker system," Magnetic Resonance Imaging, vol. 15(5), pp. 579-585, 1997.

[13] J. Weese, G. P. Penney, P. Desmedt, T. M. Buzug, D. L. G. Hill, and D. J. Hawkes, "Voxel-based 2-D/3-D registration of fluoroscopy images and CT scans for image-guided surgery," IEEE Transactions on Information Technology in Biomedicine, vol. 1(4), pp. 284-293, 1997.

[14] R. C. Susil, J. H. Anderson, and R. H. Taylor, "A single image registration method for CT guided interventions," in Medical Image Computing and ComputerAssisted Intervention - MICCAI, Berlin, Germany, 1999, pp. 798 - 808. 
[15] G. P. Penney, J. M. Blackall, M. S. Hamady, T. Sabharwal, A. Adam, and D. J. Hawkes, "Registration of freehand 3D ultrasound and magnetic resonance liver images," Medical Image Analysis, vol. 8(1), pp. 81-91, 2004.

[16] B. J. Wood, H. Zhang, A. Durrani, N. Glossop, S. Ranjan, D. Lindisch, E. Levy, F. Banovac, J. Borgert, S. Krueger, J. Kruecker, A. Viswanathan, and K. Cleary, "Navigation with electromagnetic tracking for interventional radiology procedures: A feasibility study," Journal of vascular and interventional radiology, vol. 16(4), pp. 493-505, 2005.

[17] G. Dorfman, J. Emond, B. Hamilton, J. Haller, T. Smith, and L. Clarke, "Report of the NIH/NSF Group on Image-Guided Interventions.," 2004.

[18] D. J. Hawkes, J. McClelland, C. Chan, D. L. G. Hill, K. Rhode, G. P. Penney, D. Barratt, P. J. Edwards, and J. M. Blackall, "Tissue deformation and shape models in image-guided interventions: A discussion paper," Medical Image Analysis, vol. 9(2), p. 163, 2005.

[19] J. C. Timothy, S. Maxime, M. C. David, C. B. Dean, Christine Tanner, and J. H. David, "Application of soft tissue modelling to image-guided surgery," Medical Engineering \& Physics, vol. 27(10), pp. 893-909, 2005.

[20] J. de Mey, W. Vincken, B. Op de Beeck, M. Osteaux, M. De Maeseneer, M. Noppen, M. Meysman, and M. Vanhoey, "Real time CT-fluoroscopy: Diagnostic and therapeutic applications," European Journal of Radiology, vol. 34(1), p. 32, 2000.

[21] B. D. Fornage, H. M. Kuerer, G. V. Babiera, A. N. Mirza, F. C. Ames, N. Sneige, M. I. Ross, B. S. Edeiken, and L. A. Newman, "Small $(<$ or $=2-\mathrm{cm})$ breast cancer treated with US-guided radiofrequency ablation: feasibility study," Radiology, vol. 231(Apr), p. 215, 2004.

[22] R. L. Galloway, "The process and development of image-guided procedures," Annual Review of Biomedical Engineering, vol. 3(pp. 83-108, 2001.

[23] Peters, "Image-guided surgery: From X-rays to virtual reality," Computer Methods in Biomechanics and Biomedical Engineering, vol. 4(1), p. 27, 2000.

[24] T. M. Peters, "Image-guidance for surgical procedures," Physics in Medicine \& Biology, vol. 51(14), p. R505, 2006.

[25] Philips Medical Systems, "256-slice Brilliance iCT Scanner," http://www.medical.philips.com/main/news/content/file_1650.html.

[26] GE Healthcare, "Signa OpenSpeed $0.7 T$ "

http://www.gehealthcare.com/usen/mr/open_speed/index.html.

[27] Terarecon Inc., "Aquarius iNtuition," www.terarecon.com.

[28] Vital Images Inc., "Vitrea ${ }^{\circledR}$ Software," www.vitalimages.com.

[29] M. Das, F. Sauer, U. J. Schoepf, A. Khamene, S. K. Vogt, S. Schaller, R. Kikinis, E. vanSonnenberg, and S. G. Silverman, "Augmented reality visualization for CT-guided interventions: system description, feasibility, and initial evaluation in an abdominal phantom," Radiology, vol. 240(1), p. 230, 2006.

[30] F. Sauer, "Image registration: Enabling technology for image guided surgery and therapy," in IEEE International Conference on Engineering in Medicine and Biology, 2005, pp. 7242-7245.

[31] W. Hendee, "Special Report: Biomedical Imaging Research Opportunities Workshop III. A Summary of Findings and Recommendations," Medical Physics, vol. 33(2), pp. 274-277, 2006. 
[32] J. B. A. Maintz and M. A. Viergever, "A survey of medical image registration," Medical Image Analysis, vol. 2(1), pp. 1-36, 1998.

[33] D. L. G. Hill, "Medical image registration," Physics in Medicine \& Biology, vol. 46(1), p. 1, 2001.

[34] L. Lemieux, N. D. Kitchen, S. W. Hughes, and D. G. Thomas, "Voxel-based localization in frame-based and frameless stereotaxy and its accuracy," Medical Physics, vol. 21(8), p. 1301, 1994.

[35] T. Peters, B. Davey, P. Munger, R. Comeau, A. A. Evans, and A. A. Olivier, "Three-dimensional multimodal image-guidance for neurosurgery," IEEE Transactions on Medical Imaging, vol. 15(2), pp. 121-128, 1996.

[36] C. R. Maurer, Jr., J. M. Fitzpatrick, M. Y. Wang, R. L. Galloway, Jr , R. J. Maciunas, and G. S. Allen, "Registration of head volume images using implantable fiducial markers," IEEE Transactions on Medical Imaging, vol. 16(4), pp. 447-462, 1997.

[37] R. D. Bucholz, K. R. Smith, J. M. Henderson, L. L. McDurmont, and D. W. Schulze, "Intraoperative localization using a three-dimensional optical digitizer," in Clinical Applications of Modern Imaging Technology, Los Angeles, CA, USA, 1993, pp. 312-322.

[38] S. A. Tebo, D. A. Leopold, D. M. Long, S. J. Zinreich, and D. W. Kennedy, "An optical 3D digitizer for frameless stereotactic surgery," IEEE Computer Graphics and Applications, vol. 16(1), pp. 55-64, 1996.

[39] S. Fang, R. Raghavan, and J. T. Richtsmeier, "Volume morphing methods for landmark-based 3D image deformation," in SPIE Medical Imaging 1996: Image Processing, 1996, pp. 404-415.

[40] Y. Ge, J. M. Fitzpatrick, J. R. Votaw, S. Gadamsetty, R. J. Maciunas, R. M. Kessler, and R. A. Margolin, "Retrospective registration of PET and MR brain images: an algorithm and its stereotactic validation," Journal of Computer Assisted Tomography, vol. 18(5), p. 800, 1994.

[41] D. N. Levin, C. A. Pelizzari, G. T. Chen, C. T. Chen, and M. D. Cooper, "Retrospective geometric correlation of MR, CT, and PET images," Radiology, vol. 169(3), p. 817, 1988.

[42] H. Rusinek, W. Tsui, A. V. Levy, M. E. Noz, and M. J. de Leon, "Principal Axes and Surface Fitting Methods for Three-Dimensional Image Registration," Journal of Nuclear Medicine, vol. 34(11), pp. 2019-2024, 1993.

[43] T. G. Turkington, J. M. Hoffman, R. J. Jaszczak, J. R. MacFall, C. C. Harris, C. D. Kilts, C. A. Pelizzari, and R. E. Coleman, "Accuracy of surface fit registration for PET and MR brain images using full and incomplete brain surfaces," Journal of Computer Assisted Tomography, vol. 19(1), p. 117, 1995.

[44] J. P. Thirion, "Image matching as a diffusion process: an analogy with Maxwell's demons," Medical Image Analysis, vol. 2(3), p. 243, 1998.

[45] P. M. Thompson, "A surface-based technique for warping 3-dimensional images of the brain," IEEE Transactions on Medical Imaging, vol. 15(4), p. 1, 1996.

[46] J. V. Hajnal, D. J. Hawkes, and D. L. G. Hill, Medical image registration. Boca Raton: CRC Press, 2001. 
[47] J. P. W. Pluim, J. B. A. Maintz, and M. A. Viergever, "Mutual-informationbased registration of medical images: a survey," IEEE Transactions on Medical Imaging, vol. 22(8), pp. 986-1004, 2003.

[48] M. Holden, D. L. G. Hill, E. R. E. Denton, J. M. Jarosz, T. C. Cox, T. Rohlfing, J. Goodey, and D. J. Hawkes, "Voxel similarity measures for 3-D serial MR brain image registration," IEEE Transactions on Medical Imaging, vol. 19(2), pp. 94-102, 2000.

[49] R. Shekhar and V. Zagrodsky, "Mutual information-based rigid and nonrigid registration of ultrasound volumes," IEEE Transactions on Medical Imaging, vol. 21(1), pp. 9-22, 2002.

[50] C. Davatzikos, "Nonlinear registration of brain images using deformable models," in Mathematical Methods in Biomedical Image Analysis, 1996., Proceedings of the Workshop on, 1996, pp. 94-103.

[51] B. Morten and G. Claus, "Fast Fluid Registration of Medical Images," in Proceedings of the 4th International Conference on Visualization in Biomedical Computing: Springer-Verlag, 1996.

[52] A. Hagemann, K. Rohr, H. S. Stiehl, U. Spetzger, and J. M. Gilsbach, "Biomechanical modeling of the human head for physically based, nonrigid image registration," IEEE Transactions on Medical Imaging, vol. 18(10), pp. 875-884, 1999. [53] S. K. Kyriacou, C. Davatzikos, S. J. Zinreich, and R. N. Bryan, "Nonlinear elastic registration of brain images with tumor pathology using a biomechanical model," IEEE Transactions on Medical Imaging, vol. 18(7), p. 580, 1999.

[54] J. Ashburner and K. Friston, "Nonlinear spatial normalization using basis functions," Human Brain Mapping, vol. 7(4), p. 254, 1999.

[55] B. Kim, J. Boes, K. A. Frey, and C. R. Meyer, "Mutual Information for Automated Multimodal Image Warping," in Proceedings of the 4th International Conference on Visualization in Biomedical Computing: Springer-Verlag, 1996.

[56] K. Rohr, H. S. Stiehl, R. Sprengel, B. Wolfgang, T. Buzug, J. Weese, and M. H. Kuhn, "Point-Based Elastic Registration of Medical Image Data Using Approximating Thin-Plate Splines," in Proceedings of the 4th International Conference on Visualization in Biomedical Computing: Springer-Verlag, 1996.

[57] T. Rohlfing and C. R. Maurer, Jr., "Nonrigid image registration in sharedmemory multiprocessor environments with application to brains, breasts, and bees," IEEE Transactions on Information Technology in Biomedicine, vol. 7(1), pp. 16-25, 2003.

[58] D. Rueckert, L. I. Sonoda, C. Hayes, D. L. G. Hill, M. O. Leach, and D. J. Hawkes, "Nonrigid registration using free-form deformations: application to breast MR images," IEEE Transactions on Medical Imaging, vol. 18(8), pp. 712-721, 1999. [59] J. F. Krucker, G. L. LeCarpentier, J. B. Fowlkes, and P. L. Carson, "Rapid elastic image registration for 3-D ultrasound," IEEE Transactions on Medical Imaging, vol. 21(11), pp. 1384-1394, 2002.

[60] V. Walimbe and R. Shekhar, "Automatic elastic image registration by interpolation of 3D rotations and translations from discrete rigid-body transformations," Medical Image Analysis, vol. 10(6), p. 899, 2006. 
[61] A. Collignon, F. Maes, D. Delaere, D. Vandermeulen, P. Suetens, and G. Marchal, "Automated multi-modality image registration based on information theory," in Information Processing in Medical Imaging, 1995, pp. 263-274.

[62] W. M. Wells, P. Viola, H. Atsumi, S. Nakajima, and R. Kikinis, "Multi-modal volume registration by maximization of mutual information," Medical Image Analysis, vol. 1(1), pp. 35-51, 1996.

[63] M. Capek, L. mroz, and R. Wegenkittl, "Robust and fast medical registration of 3D-multi-modality data sets," Mediterranean Conference on Medical and Biological Engineering and Computing, p. 515, 2001.

[64] C. Studholme, D. L. G. Hill, and D. J. Hawkes, "An overlap invariant entropy measure of 3D medical image alignment," Pattern Recognition, vol. 32(1), pp. 71-86, 1999.

[65] W. H. Press, Numerical recipes in $C++$ : the art of scientific computing, 2nd ed. Cambridge, UK ; New York: Cambridge University Press, 2002.

[66] R. Shekhar, V. Walimbe, S. Raja, V. Zagrodsky, M. Kanvinde, G. Y. Wu, and B. Bybel, "Automated 3-dimensional elastic registration of whole-body PET and CT from separate or combined scanners," Journal of Nuclear Medicine, vol. 46(9), pp. 1488-1496, Sep 2005.

[67] V. Walimbe, V. Zagrodsky, S. Raja, B. Bybel, M. Kanvinde, and R. Shekhar, "Elastic registration of three-dimensional whole body CT and PET images by quaternion-based interpolation of multiple piecewise linear rigid-body registrations," in Proceedings of SPIE Medical Imaging, 2004, pp. 119-128.

[68] S. Clippe, D. Sarrut, C. Malet, S. Miguet, C. Ginestet, and C. Carrie, "Patient setup error measurement using 3D intensity-based image registration techniques," International Journal of Radiation Oncology, Biology, and Physics, vol. 56(1), pp. 259-265, 2003.

[69] F. Maes, A. Collignon, D. Vandermeulen, G. Marchal, and P. Suetens, "Multimodality image registration by maximization of mutual information," IEEE Transactions on Medical Imaging,, vol. 16(2), pp. 187-198, 1997.

[70] Z. Hongying, Z. Xiaozhou, S. Jizhou, and J. Zhang, "A novel medical image registration method based on mutual information and genetic algorithm," in International Conference on Computer Graphics, Imaging and Vision: New Trends, 2005, pp. 221-226.

[71] J. M. Rouet, J. J. Jacq, and C. Roux, "Genetic algorithms for a robust 3-D MRCT registration," IEEE transactions on information technology in biomedicine, vol. 4(2), p. 126, 2000.

[72] C. Nikou, F. Heitz, J. Armspach, I. Namer, and D. Grucker, "Registration of MR/MR and MR/SPECT Brain Images by Fast Stochastic Optimization of Robust Voxel Similarity Measures," NeuroImage, vol. 8(1), pp. 30-43, 1998.

[73] N. Ritter, R. Owens, J. Cooper, R. H. Eikelboom, and P. P. Van Saarloos, "Registration of stereo and temporal images of the retina," IEEE Transactions on Medical Imaging, vol. 18(5), pp. 404-418, 1999.

[74] A. Carrillo, J. L. Duerk, J. S. Lewin, and D. L. Wilson, "Semiautomatic 3-D image registration as applied to interventional MRI liver cancer treatment," IEEE Transactions on Medical Imaging, vol. 19(3), pp. 175-185, 2000. 
[75] H. Mark, A. S. Julia, and L. G. H. Derek, "Quantifying Small Changes in Brain Ventricular Volume Using Non-rigid Registration," in Proceedings of the 4th International Conference on Medical Image Computing and Computer-Assisted Intervention: Springer-Verlag, 2001.

[76] K. Z. Abd-Elmoniem, A. M. Youssef, and Y. M. Kadah, "Real-time speckle reduction and coherence enhancement in ultrasound imaging via nonlinear anisotropic diffusion," IEEE Transactions on Biomedical Engineering, vol. 49(9), pp. 997-1014, 2002.

[77] O. Dandekar, K. Siddiqui, V. Walimbe, and R. Shekhar, "Image registration accuracy with low-dose CT: How low can we go?," in IEEE International Symposium on Biomedical Imaging, 2006, pp. 502-505.

[78] P. Lei, O. Dandekar, D. Widlus, P. Malloy, and R. Shekhar, "Incorporation of PET into CT-Guided Liver Radiofrequency Ablation," Radiology (under revision), 2008.

[79] M. Kachelriess, O. Watzke, and W. A. Kalender, "Generalized multidimensional adaptive filtering for conventional and spiral single-slice, multi-slice, and cone-beam CT," Medical Physics, vol. 28(4), pp. 475-490, 2001.

[80] L. Keselbrener, Y. Shimoni, and S. Akselrod, "Nonlinear filters applied on computerized axial tomography: Theory and phantom images," Medical Physics, vol. 19(4), pp. 1057-1064, 1992.

[81] P. Perona and M. Jitendra, "Scale-space and edge detection using anisotropic diffusion," IEEE Transactions on Pattern Analysis and Machine Intelligence, vol. 12(7), pp. 629-639, July 19901990.

[82] R. T. Whitaker and S. M. Pizer, "A multi-scale approach to nonuniform diffusion," CVGIP: Image Understanding, vol. 57(1), pp. 99-110, 1993.

[83] A. Dorati, C. Lamberti, A. Sarti, P. Baraldi, and R. Pini, "Pre-processing for 3D echocardiography," Computers in Cardiology, pp. 565-568, 1995.

[84] M. A. Cantin, Y. Savaria, and P. Lavoie, "A comparison of automatic word length optimization procedures," in IEEE International Symposium on Circuits and Systems, 2002, pp. 612-615.

[85] T. J. Todman, G. A. Constantinides, S. J. E. Wilton, O. Mencer, W. Luk, and P. Y. K. Cheung, "Reconfigurable computing: architectures and design methods," IEE Proceedings - Computers and Digital Techniques, vol. 152(2), pp. 193-207, 2005.

[86] G. A. Constantinides, P. Y. K. Cheung, and W. Luk, "Wordlength optimization for linear digital signal processing," IEEE Transactions on Computer-Aided Design of Integrated Circuits and Systems, vol. 22(10), pp. 1432-1442, 2003.

[87] A. Nayak, M. Haldar, A. Choudhary, and P. A. B. P. Banerjee, "Precision and error analysis of MATLAB applications during automated hardware synthesis for FPGAs," in Proceedings of Design, Automation and Test in Europe, 2001, pp. 722728.

[88] A. V. Oppenheim, R. W. Schafer, and J. R. Buck, Discrete-time signal processing, 2nd ed. Upper Saddle River, N.J.: Prentice Hall, 1999.

[89] M. Stephenson, J. Babb, and S. Amarasinghe, "Bidwidth analysis with application to silicon compilation," ACM SIGPLAN Conference on Programming Language Design and Implementation, vol. 35(5), pp. 108-120, 2000. 
[90] S. A. Wadekar and A. C. Parker, "Accuracy sensitive word-length selection for algorithm optimization," in Proceedings of International Conference on Computer Design: VLSI in Computers and Processors, ICCD '98. , 1998, pp. 54-61.

[91] K. Han and B. Evans, "Optimum wordlength search using sensitivity information," EURASIP Journal on Applied Signal Processing vol. 2006, Article ID 92849, pp. 1-14, 2006.

[92] W. Sung and K. Kum, "Simulation-based word-length optimization method for fixed-point digital signal processing systems," IEEE Transactions on Signal Processing, vol. 43(12), pp. 3087-3090, 1995.

[93] H. Choi and W. P. Burleson, "Search-based wordlength optimization for VLSI/DSP synthesis," in Proceedings of IEEE Workshop on VLSI Signal Processing, VII, 1994, pp. 198-207.

[94] M. Leban and J. F. Tasic, "Word-length optimization of LMS adaptive FIR filters," in 10th Mediterranean Electrotechnical Conference 2000, pp. 774-777.

[95] T. Givargis, F. Vahid, and J. Henkel, "System-level exploration for Paretooptimal configurations in parameterized system-on-a-chip," IEEE Transactions on Very Large Scale Integration (VLSI) Systems, vol. 10(4), pp. 416-422, 2002.

[96] K. Kum, J. Kang, and W. Sung, "AUTOSCALER for C: an optimizing floatingpoint to integer $\mathrm{C}$ program converter for fixed-point digital signal processors," IEEE Transactions on Circuits and Systems II: Analog and Digital Signal Processing, vol. 47(9), pp. 840-848, 2000.

[97] P. M. Dew, R. A. Earnshaw, and T. R. Heywood, Parallel processing for computer vision and display. Workingham, England ; Reading, Mass.: AddisonWesley, 1989.

[98] V. K. Prasanna Kumar, Parallel architectures and algorithms for image understanding. Boston: Academic Press, 1991.

[99] A. Bruhn, T. Jakob, M. Fischer, T. Kohlberger, J. Weickert, U. Bruning, and C. Schnorr, "Designing 3-D nonlinear diffusion filters for high performance cluster computing," Proc. of the 24th DAGM Symposium on Pattern Recognition, vol. 2449(pp. 290-297, 2002.

[100] A. Bruhn, T. Jakob, M. Fischer, T. Kohlberger, J. Weickert, U. Bruning, and C. Schnorr, "High performance cluster computing with 3-D nonlinear diffusion filters," Real-Time Imaging, vol. 10(1), pp. 41-51, 2004.

[101] S. Tabik, E. M. Garzon, I. Garcia, and J. J. Fernandez, "Evaluation of parallel paradigms on Anisotropic Nonlinear Diffusion," in 12th International Euro-Par Conference, 2006, pp. 1159-1168.

[102] M. Karaman and L. Onural, "New radix-2-based algorithm for fast median filtering," Electronics Letters, vol. 25(11), pp. 723-724, 1989.

[103] K. Oflazer, "Design and implementation of a single-chip 1- D median filter," IEEE Transactions on Acoustics, Speech and Signal Processing, vol. 31(5), pp. 11641168, 1983.

[104] E. Ataman and E. Alparslan, "Applications of median filtering algorithm to images," Electronics Division, Marmara Research Institute, Gebze, Turkey 1978.

[105] J. P. Fitch, E. J. Coyle, and N. C. J. Gallagher, "Median filtering by threshold decomposition," IEEE Transactions on Acoustics, Speech, and Signal Processing, vol. 32(6), pp. 1183-1188, 1984. 
[106] C. L. Lee and C. W. Jen, "Bit-sliced median filter design based on majority gate," IEE Proceedings, Part G: Circuits, Devices and Systems, vol. 139(1), pp. 63$71,1992$.

[107] I. Hatirnaz, F. K. Gurkaynak, and Y. Leblebici, "A compact modular architecture for the realization of high-speed binary sorting engines based on rank ordering," Proc. of the IEEE International Symposium on Circuits and Systems, ISCAS, vol. 4(pp. 685-688, 2000.

[108] A. A. Hiasat, M. M. Al-Ibrahim, and K. M. Gharaibeh, "Design and implementation of a new efficient median filtering algorithm," IEE Proceedings on Vision, Image and Signal Processing, vol. 146(5), pp. 273-278, 1999.

[109] B. K. Kar, K. M. Yusuf, and D. K. Pradhan, "Bit-serial generalized median filters," Proc. of the IEEE International Symposium on Circuits and Systems, ISCAS, vol. 3(pp. 85-88, 1994.

[110] C. Chakrabarti, "High sample rate array architectures for median filters," IEEE Transactions on Signal Processing, vol. 42(3), pp. 707-712, 1994.

[111] L. W. Chang and J. H. Lin, "A bit-level systolic array for median filter," IEEE Transactions on Signal Processing, vol. 40(8), pp. 2079-2083, 1992.

[112] K. Chen, "An integrated bit-serial 9-point median chip," Proc. of the European Conference on Circuit Theory and Design, pp. 339-343, 1989.

[113] R. Roncella, R. Saletti, and P. Terreni, "70-MHz 2-um CMOS bit-level systolic array median filter," IEEE Journal of Solid-State Circuits, vol. 28(5), pp. 530-536, 1993.

[114] W. Plishker, O. Dandekar, S. Bhattacharyya, and R. Shekhar, "A taxonomy for medical image registration acceleration techniques," in IEEE Life Science Systems and Applications Workshop, 2007, pp. 160-163.

[115] S. Ourselin, R. Stefanescu, and X. Pennec, "Robust registration of multi-modal images: Towards real-time clinical applications " in Medical Image Computing and Computer-Assisted Intervention - MICCAI, 2002, pp. 140-147.

[116] R. Stefanescu, X. Pennec, and N. Ayache, "Parallel non-rigid registration on a cluster of workstations," Proceedings of HealthGrid, 2003.

[117] F. Ino, K. Ooyama, and K. Hagihara, "A data distributed parallel algorithm for nonrigid image registration," Parallel Computing, vol. 31(1), p. 19, 2005.

[118] S. K. Warfield, F. Talos, A. Tei, A. Bharatha, A. Nabavi, M. Ferrant, P. M. Black, F. Jolesz, and R. Kikinis, "Real-time registration of volumetric brain MRI by biomechanical simulation of deformation during image guided neurosurgery," Computing and Visualization in Science, vol. 5(1), pp. 3-11, 2002.

[119] R. Strzodka, M. Droske, and M. Rumpf, "Fast Image Registration in DX9 graphics Hardware," Journal of Medical Informatics and Technologies, vol. 6(1), pp. 43-49, 2003.

[120] A. Köhn, J. Drexl, F. Ritter, M. König, and H. Peitgen, "GPU Accelerated Image Registration in Two and Three Dimensions," in Bildverarbeitung für die Medizin, 2006, pp. 261-265.

[121] W. Plishker, O. Dandekar, S. Bhattacharyya, and R. Shekhar, "Towards a heterogeneous medical image registration acceleration platform," Proceedings of the IEEE Biomedical Circuits and Systems Conference, pp. 231-234, 2007. 
[122] C. Vetter, C. Guetter, C. Xu, and R. Westermann, "Non-rigid multi-modal registration on the GPU," in SPIE Medical Imaging 2007: Image Processing, 2007, p. 651228.

[123] M. Ohara, H. Yeo, F. Savino, G. Iyengar, L. Gong, H. Inoue, H. Komatsu, V. Sheinin, and S. Daijavad, "Accelerating mutual-information-based linear registration on the cell broadband engine processor," in IEEE International Conference on Multimedia and Expo, 2007, pp. 272-275.

[124] J. Jiang, W. Luk, and D. Rueckert, "FPGA-based computation of free-form deformations in medical image registration," in proceedings of IEEE International Conference on Field-Programmable Technology (FPT), 2003, pp. 234-241.

[125] H. Keding, M. Willems, M. Coors, and H. A. M. H. Meyr, "FRIDGE: a fixedpoint design and simulation environment," in Proceedings of Design, Automation and Test in Europe, 1998, pp. 429-435.

[126] G. A. Constantinides, P. Y. K. Cheung, and W. Luk, "Optimum and heuristic synthesis of multiple word-length architectures," IEEE Transactions on Very Large Scale Integration (VLSI) Systems, vol. 13(1), pp. 39-57, 2005.

[127] S. Kim, K. Kum, and W. Sung, "Fixed-point optimization utility for C and C++ based digital signal processing programs," IEEE Transactions on Circuits and Systems II: Analog and Digital Signal Processing, vol. 45(11), pp. 1455-1464, 1998.

[128] K. Kum and W. Sung, "Combined word-length optimization and high-level synthesis of digital signal processing systems," IEEE Transactions on ComputerAided Design of Integrated Circuits and Systems, vol. 20(8), pp. 921-930, 2001.

[129] I. Das and J. E. Dennis, "A closer look at drawbacks of minimizing weighted sums of objectives for Pareto set generation in multicriteria optimization problems," Structural and Multidisciplinary Optimization, vol. 14(1), pp. 63-69, 1997.

[130] M. S. Bright and T. Arslan, "Synthesis of low-power DSP systems using a genetic algorithm," IEEE Transactions on Evolutionary Computation, vol. 5(1), pp. 27-40, 2001.

[131] C. L. Valenzuela and P. Y. Wang, "VLSI placement and area optimization using a genetic algorithm to breed normalized postfix expressions," IEEE Transactions on Evolutionary Computation, vol. 6(4), pp. 390-401, 2002.

[132] G. Antoch, J. F. Debatin, J. Stattaus, H. Kuehl, and F. M. Vogt, "Value of CT volume imaging for optimal placement of radiofrequency ablation probes in liver lesions," Journal of Vascular and Interventional Radiology, vol. 13(11), p. 1155, 2002.

[133] D. E. Dupuy and S. N. Goldberg, "Image-guided radiofrequency tumor ablation: challenges and opportunities--part II," Journal of vascular and interventional radiology, vol. 12(10), pp. 1135-1148, 2001.

[134] N. S. Goldberg and D. E. Dupuy, "Image-guided radiofrequency tumor ablation: challenges and opportunities--part I," Journal of vascular and interventional radiology, vol. 12(9), pp. 1021-1032, 2001.

[135] J. R. Haaga, "Interventional CT: 30 years' experience," European radiology, vol. 15(p. D116, 2005.

[136] I. Viola, A. Kanitsar, and M. E. Groller, "Hardware-based nonlinear filtering and segmentation using high-level shading languages," IEEE Visualization, p. 309, 2003. 
[137] M. Jiang and D. Crookes, "High-performance 3D median filter architecture for medical image despeckling," Electronics Letters, vol. 42(24), p. 1379, 2006.

[138] G. Gerig, O. Kubler, R. Kikinis, and F. A. Jolesz, "Nonlinear Anisotropic Filtering of MRI Data," IEEE Transactions on Medical Imaging, vol. 11(2), pp. 221232, 1992.

[139] C. R. Castro-Pareja, J. M. Jagadeesh, and R. Shekhar, "FAIR: A Hardware Architecture for Real-Time 3-D Image Registration," IEEE Transactions on Information Technology in Biomedicine, vol. 7(4), pp. 426-434, 2003.

[140] M. Doggett and M. Meissner, "A memory addressing and access design for real time volume rendering," IEEE International Symposium on Circuits and Systems, ISCAS, vol. 4(pp. 344-347, 1999.

[141] H. Pfister, "Archtectures for real-time volume rendering," Future Generation Computer Systems, vol. 15(1), pp. 1-9, Feb. 19991999.

[142] H. Pfister and A. Kaufman, "Cube-4: A scalable architecture for real-time volume rendering," Proc. of the 1996 symposium on volume visualization, pp. 47-54, 1996.

[143] C. R. Castro-Pareja, O. S. Dandekar, and R. Shekhar, "FPGA-based real-time anisotropic diffusion filtering of 3D ultrasound images," in Real-Time Imaging, 2005, pp. 123-131.

[144] O. Dandekar, C. Castro-Pareja, and R. Shekhar, "FPGA-based real-time 3D image preprocessing for image-guided medical interventions," Journal of Real-Time Image Processing, vol. 1(4), pp. 285-301, 2007.

[145] X. Li and T. Chen, "Nonlinear diffusion with multiple edginess thresholds," Pattern Recognition, vol. 27(8), pp. 1029-1037, 1994.

[146] F. J. Gallegos-Funes and V. I. Ponomaryov, "Real-time image filtering scheme based on robust estimators in presence of impulsive noise," Real-Time Imaging, vol. 10(2), p. 69, 2004.

[147] D. Mattes, D. R. Haynor, H. Vesselle, T. K. Lewellen, and W. Eubank, "PETCT image registration in the chest using free-form deformations," IEEE Transactions on Medical Imaging, vol. 22(1), pp. 120-128, 2003.

[148] P. Lei, O. Dandekar, F. Mahmoud, D. Widlus, P. Malloy, and R. Shekhar, "PET guidance for liver radiofrequency ablation: an evaluation," SPIE Medical Imaging 2007: Visualization and Image-Guided Procedures, p. 650918, 2007.

[149] R. Shekhar, O. Dandekar, S. Kavic, I. George, R. Mezrich, and A. Park, "Development of continuous CT-guided minimally invasive surgery," in SPIE Medical Imaging 2007: Visualization and Image-Guided Procedures, 2007, pp. 65090D-8.

[150] V. Walimbe, O. Dandekar, F. Mahmoud, and R. Shekhar, "Automated 3D elastic registration for improving tumor localization in whole-body PET-CT from combined scanner," in IEEE Annual International Conference on Engineering in Medicine and Biology, 2006, pp. 2799-2802.

[151] J. Wu, O. Dandekar, V. Walimbe, W. D'Souza, and R. Shekhar, "Automatic prostate localization using elastic registration of planning CT and daily 3D ultrasound images," in SPIE Medical Imaging 2007: Visualization and Image-Guided Procedures, 2007, p. 650913. 
[152] H. Hassler and N. Takagi, "Function evaluation by table look-up and addition," in IEEE Symposium on Computer Arithmetic, IEEE, 1995, p. 10.

[153] D. M. Mandelbaum and S. G. Mandelbaum, "A fast, efficient parallel-acting method of generating functions defined by power series, including logarithm, exponential, and sine, cosine," IEEE Transactions on Parallel and Distributed Systems, vol. 7(1), p. 33, 1996.

[154] S. L. SanGregory, C. Brothers, D. Gallagher, and R. Siferd, "A fast, low-power logarithm approximation with CMOS VLSI implementation," in IEEE 42nd Midwest Symposium on Circuits and Systems, 2000, p. 91.

[155] C. R. Castro-Pareja and R. Shekhar, "Hardware acceleration of mutual information-based 3D image registration," Journal of Imaging Science and Technology, vol. 49(2), pp. 105-113, 2005.

[156] C. R. Castro-Pareja and R. Shekhar, "Adaptive reduction of intensity levels in 3D images for mutual information-based registration," in SPIE Medical Imaging 2005: Image Processing, San Diego, CA, USA, 2005, p. 1201.

[157] O. Dandekar and R. Shekhar, "FPGA-Accelerated Deformable Image Registration for Improved Target-Delineation During CT-Guided Interventions," IEEE Transactions on Biomedical Circuits and Systems, vol. 1(2), pp. 116-127, 2007. [158] G. A. Constantinides, P. Y. K. Cheung, and W. Luk, "The Multiple Wordlength Paradigm," in The 9th Annual IEEE Symposium on Field-Programmable Custom Computing Machines, FCCM '01. , 2001, pp. 51-60.

[159] G. A. Constantinides and G. J. Woeginger, "The complexity of multiple wordlength assignment," Applied Mathematics Letters, vol. 15(2), pp. 137-140, 2002. [160] E. Zitzler, M. Laumanns, and L. Thiele, "SPEA2: Improving the Strength Pareto Evolutionary Algorithm for Multiobjective Optimization," in EUROGEN 2001 - Evolutionary Methods for Design, Optimisation and Control with Applications to Industrial Problems, 2001, pp. 95-100.

[161] K. Deb, A. Pratap, S. Agarwal, and T. A. M. T. Meyarivan, "A fast and elitist multiobjective genetic algorithm: NSGA-II," IEEE Transactions on Evolutionary Computation, vol. 6(2), pp. 182-197, 2002.

[162] Altera Corp., "Altera IP Megacore Library," http://www.altera.com/literature/lit-ip.jsp.

[163] W. Luk, S. Guo, N. Shirazi, and N. Zhuang, "A framework for developing parametrised FPGA libraries," in Field-Programmable Logic Smart Applications, New Paradigms and Compilers, 1996, pp. 24-33.

[164] W. Luk and S. McKeever, "Pebble: A Language For Parametrised and Reconfigurable Hardware Design," in Field-Programmable Logic and Applications From FPGAs to Computing Paradigm, 1998, p. 9.

[165] Xilinx Inc., "Xilinx Core Generator," http://www.xilinx.com/ise/products/coregen_overview.pdf.

[166] J. Zhao, W. Chen, and S. Wei, "Parameterized IP core design," in Proceedings of 4th International Conference on ASIC, 2001, pp. 744-747.

[167] T. Back, U. Hammel, and H. P. Schwefel, "Evolutionary computation: comments on the history and current state," IEEE Transactions on Evolutionary Computation, vol. 1(1), pp. 3-17, 1997. 
[168] V. Kianzad and S. S. Bhattacharyya, "Efficient techniques for clustering and scheduling onto embedded multiprocessors," IEEE Transactions on Parallel and Distributed Systems, vol. 17(7), pp. 667-680, 2006.

[169] G. A. Constantinides, P. Y. K. Cheung, and W. Luk, "Optimum wordlength allocation," in Proceedings of 10th Annual IEEE Symposium on Field-Programmable Custom Computing Machines, 2002, pp. 219-228.

[170] E. Zitzler and L. Thiele, "Multiobjective evolutionary algorithms: a comparative case study and the strength Pareto approach," IEEE Transactions on Evolutionary Computation, vol. 3(4), pp. 257-271, 1999.

[171] A. J. Bilchik, D. M. Rose, D. P. Allegra, P. J. Bostick, E. Hsueh, and D. L. Morton, "Radiofrequency ablation: A minimally invasive technique with multiple applications," Cancer Journal from Scientific American, vol. 5(6), p. 356, 1999.

[172] J. H. Morgan, G. M. Royer, P. Hackett, T. C. Gamblin, B. L. McCampbell, A. Conforti, and P. S. Dale, "Radio-frequency ablation of large, nonresectable hepatic tumors," The American surgeon, vol. 70(12), p. 1035, 2004.

[173] D. M. Rose, D. P. Allegra, P. J. Bostick, L. J. Foshag, and A. J. Bilchik, "Radiofrequency ablation: A novel primary and adjunctive ablative technique for hepatic malignancies," American Surgeon, vol. 65(11), p. 1009, 1999.

[174] Toshiba, "The Next Revolution: 256-Slice CT," http://madeforlife.toshiba.com/ct256/TMS082_CT_WP_256.pdf.

[175] R. Bellotti, F. De Carlo, G. Gargano, S. Tangaro, D. Cascio, E. Catanzariti, P. Cerello, S. C. Cheran, P. Delogu, I. De Mitri, C. Fulcheri, D. Grosso, A. Retico, S. Squarcia, E. Tommasi, and B. Golosio, "A CAD system for nodule detection in lowdose lung CTs based on region growing and a new active contour model," Medical Physics, vol. 34(12), pp. 4901-4910, 2007.

[176] D. S. Gierada, T. K. Pilgram, M. Ford, R. M. Fagerstrom, T. R. Church, H. Nath, K. Garg, and D. C. Strollo, "Lung Cancer: Interobserver Agreement on Interpretation of Pulmonary Findings at Low-Dose CT Screening," Radiology, vol. 246(1), pp. 265-272, 2007.

[177] D. Tack, P. Bohy, I. Perlot, V. De Maertelaer, O. Alkeilani, S. Sourtzis, and P. A. Gevenois, "Suspected Acute Colon Diverticulitis: Imaging with Low-Dose Unenhanced Multi-Detector Row CT," Radiology, vol. 237(1), pp. 189-196, 2005.

[178] D. Sennst, M. Kachelriess, C. Leidecker, B. Schmidt, O. Watzke, and W. A. Kalender, "An Extensible Software-based Platform for Reconstruction and Evaluation of CT Images," Radiographics, vol. 24(2), pp. 601-613, 2004.

[179] F. Richard, "Smooth interpolation of scattered data by local thin plate splines," Computers \&amp; Mathematics with Applications, vol. 8(4), p. 273, 1982.

[180] D. G. Gobbi and T. M. Peters, "Generalized 3D nonlinear transformations for medical imaging: an object-oriented implementation in VTK," Computerized Medical Imaging and Graphics, vol. 27(4), pp. 255-265, 2003.

[181] C. R. Meyer, J. L. Boes, B. Kim, P. H. Bland, K. R. Zasadny, P. V. Kison, K. Koral, K. A. Frey, and R. L. Wahl, "Demonstration of accuracy and clinical versatility of mutual information for automatic multimodality image fusion using affine and thin-plate spline warped geometric deformations," Medical Image Analysis, vol. 1(3), pp. 195-206, 1997. 
[182] S. Balocco, O. Basset, C. Cachard, and P. Delachartre, "Spatial anisotropic diffusion and local time correlation applied to segmentation of vessels in ultrasound image sequences," Proc. of the IEEE Symposium on Ultrasonics, vol. 2(pp. 15491552, 2003.

[183] F. Xu, Y. Yu, S. T. Acton, and J. A. Hossack, "Detection of myocardinal boundaries from ultrasound imagery using activecontours," Proc. of the IEEE Symposium on Ultrasonics, vol. 2(pp. 1537-1540, 2003.

[184] R. Shekhar, O. Dandekar, S. Kavic, I. George, R. Mezrich, and A. Park, "Development of continuous CT-guided minimally invasive surgery," Multimedia Meets Virtual Reality (MMVR), 2007.

[185] C. D. Knox, C. D. Anderson, L. W. Lamps, R. B. Adkins, and C. W. Pinson, "Long-term survival after resection for primary hepatic carcinoid tumor," Annals of Surgical Oncology, vol. 10(10), pp. 1171-1175, Dec 2003.

[186] P. Veit, G. Antoch, H. Stergar, A. Bockisch, M. Forsting, and H. Kuehl, "Detection of residual tumor after radiofrequency ablation of liver metastasis with dual-modality PET/CT: initial results," European Radiology, vol. 16(1), pp. 80-87, Jan 2006.

[187] A. R. Gillams, "Liver ablation therapy," British Journal of Radiology, vol. 77(921), pp. 713-723, Sep 2004.

[188] J. P. McGhana and G. D. Dodd, 3rd, "Radiofrequency ablation of the liver: current status," American Journal of Roentgenology, vol. 176(1), pp. 3-16, 2001. [189] M. S. Chen, J. Q. Li, Y. Zheng, R. P. Guo, H. H. Liang, Y. Q. Zhang, X. J. Lin, and W. Y. Lau, "A prospective randomized trial comparing percutaneous local ablative therapy and partial hepatectomy for small hepatocellular carcinoma," Annals of Surgery, vol. 243(3), pp. 321-328, 2006.

[190] L. Solbiati, T. Livraghi, S. N. Goldberg, T. Ierace, F. Meloni, M. Dellanoce, L. Cova, E. F. Halpern, and G. S. Gazelle, "Percutaneous radio-frequency ablation of hepatic metastases from colorectal cancer: long-term results in 117 patients," Radiology, vol. 221(1), pp. 159-166, 2001.

[191] C. D. Roman, W. H. Martin, and D. Delbeke, "Incremental value of fusion imaging with integrated PET-CT in oncology," Clinical Nuclear Medicine, vol. 30(7), pp. 470-477, 2005.

[192] K. K. Ng, C. M. Lam, R. T. Poon, V. Ai, W. K. Tso, and S. T. Fan, "Thermal ablative therapy for malignant liver tumors: a critical appraisal," Journal of Gastroenterology and Hepatology, vol. 18(6), pp. 616-629, 2003.

[193] H. Higgins and D. L. Berger, "RFA for liver tumors: Does it really work?," Oncologist, vol. 11(7), pp. 801-808, 2006.

[194] S. B. Solomon, "Incorporating CT, MR imaging, and positron emission tomography into minimally invasive therapies," Journal of Vascular and Interventional Radiology, vol. 16(4), pp. 445-447, 2005.

[195] S. Venugopal, C. R. Castro-Pareja, and O. S. Dandekar, "An FPGA-based 3D image processor with median and convolution filters for real-time applications," in Real-Time Imaging, 2005, pp. 174-182. 